\title{
Traction-separation law for CFRP-concrete interface fracture under mode II loading
}

\author{
Fatemeh Sedigh Imani \\ West Virginia University
}

Follow this and additional works at: https://researchrepository.wvu.edu/etd

\section{Recommended Citation}

Sedigh Imani, Fatemeh, "Traction-separation law for CFRP-concrete interface fracture under mode II loading" (2010). Graduate Theses, Dissertations, and Problem Reports. 2134.

https://researchrepository.wvu.edu/etd/2134

This Thesis is protected by copyright and/or related rights. It has been brought to you by the The Research Repository @ WVU with permission from the rights-holder(s). You are free to use this Thesis in any way that is permitted by the copyright and related rights legislation that applies to your use. For other uses you must obtain permission from the rights-holder(s) directly, unless additional rights are indicated by a Creative Commons license in the record and/ or on the work itself. This Thesis has been accepted for inclusion in WVU Graduate Theses, Dissertations, and Problem Reports collection by an authorized administrator of The Research Repository @ WVU. For more information, please contact researchrepository@mail.wvu.edu. 
Traction-Separation Law for CFRP-Concrete Interface Fracture under Mode II Loading

Fatemeh Sedigh Imani

Thesis submitted to the College of Engineering and Mineral Resources

at West Virginia University in partial fulfillment of the requirements

for the degree of

\author{
Master of Science \\ in \\ Mechanical Engineering
}

Julio F. Davalos, Ph.D., Chair

An Chen, Ph.D., Co-Chair

Indrajit Ray, Ph.D.

Ever Barbero, Ph.D.

Department of Mechanical and Aerospace Engineering

Morgantown, West Virginia

2010

Keywords: Fracture, Interface, Single Shear Test, Durability, CFRPConcrete, J-Integral 


\section{ABSTRACT \\ Experimental Determination of Traction-Separation Law for FRP-Concrete Interface under Mode II}

\section{Fatemeh Sedigh Imani}

The rehabilitation and retrofitting of concrete structural members using externally bonded Fiber Reinforced Polymer (FRP) strips has been steadily gaining use in recent years due to its many advantages, such as ease and speed of construction, low cost and maintenance, and high strength/weight ratio. An important design issue with significant performance and safety implications is the debonding of externally bonded FRP strips in flexural members, where the delamination is primarily due to Mode II facture. A significant amount of research has been conducted in this area, but there are concerns about interface durability. This study is based upon a fracture mechanics approach using Mode II single-shear tests to evaluate the durability of Carbon FRP (CFRP)-concrete interface subject to two combined environmental conditioning variables: (1) immersion in deionized water varying from 0 to 15 weeks; and simultaneously (2) controlled temperatures varying from $77^{\circ} \mathrm{F}$ to $140^{\circ} \mathrm{F}\left(25^{\circ} \mathrm{C}\right.$ to $\left.60^{\circ} \mathrm{C}\right)$ of the same samples immersed in water. A new method is proposed based on J-integral to obtain the fracture energy release rate and the traction-separation law, by measuring the load and slip at the debonding end only, which was verified by the traditional strain-based method. The durability of the interface is characterized by the energy release rate (ERR). By comparing the results with those from unconditioned companion specimens, it is found that considerable degradation of the interface integrity resulted with increased moisture duration and temperature. Representative delaminated FRP specimens were further studied using CMM (Coordinate Measuring Machine) and optical microscopy to obtain a qualitative understanding of the fractured surface. These results were in accord with the fracture testing results. 
To my brother, Amir, who will always be my best friend and role model, despite the fact that he threw the watermelon. 


\section{Acknowledgements:}

Among all who have contributed to my education at West Virginia University, my greatest appreciation without doubt belongs to Professor Julio F. Davalos, my advisor and mentor for the past twenty-eight months. His intelligence, expertise, wit, and encouraging personality was the driving force behind each step of this journey.

I would not have been able to complete this work, were it not for the support of the WVU Departments of Civil and Environmental Engineering, and Mechanical and Aerospace Engineering. Specifically, I would like to thank my co-advisors Dr. Indrajit Ray, and Dr. An Chen, who created a warm, friendly research environment that always brought out the best in me, academically, personally, and professionally. I am grateful for Dr. Ray, for his guidance and for sharing his wealth of knowledge of concrete and the microstructural study of the fractured laminates.

I would also like to thank Dr. Barbero, Chairman of MAE department, throughout the course of the bulk of my studies at WVU. His expertise on composites is a great addition to my committee, and I am grateful for the opportunity to have benefited from his teaching on a personal level.

I must also acknowledge my student aides and graduate students: John Wattick, Krishna Chandra Nandam, James Barnhart, Adam Burnley, Sathish Konduru, Arkamitra Kar, Manish Roy, Matthew Anderson, Adam Justice, Soumya Sundar Chowdhury, and Mehrzad Zahabi. Without their help and hard work, the completion of this project would have not been possible. The future holds a great deal of promise and is bright for each of these talented students.

I must also thank technicians Jared Grim, Jerry Nestor, Charles Coleman, and David Turner for their valuable experience and problem solving skills. Special thanks to Mark Skidmore for making it all possible by kindly providing the high level board.

I thank my friends for the hours of conversations when concrete and composites were not the topic of discussion. I must sincerely thank two special friends; I thank Solmaz Ziad for all her kind support over the past six months, among which the smallest contribution to my life was editing my thesis. I am forever grateful to Hooshang Aban, for being my guiding light in keeping

things in perspective, and for reminding me that fighting for what you believe in is always more important than any degree of personal success. 
Last but certainly not the least; I am eternally grateful for my dear family. Not a day goes by that I am not thankful for my mother for sacrificing it all for her children and for teaching us by example. My father will always have a special place in my heart, and with each life lesson, I am reminded of how he always wanted the best for me. My quest for forgiveness has taught me everything I have ever needed to know in life. I hope that I have made him proud, as he has always been a source of pride and honor to me. And Amir, I wouldn't have been where I am today if it wasn't for you. You have taught me the true meaning of unconditional love and full support. This work is as much mine as it is yours. 


\section{Table of Contents:}

\section{Introduction ...............................................................1 1}

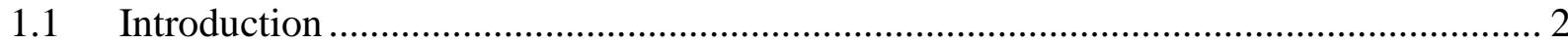

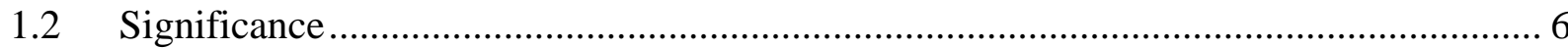

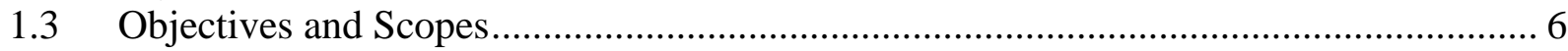

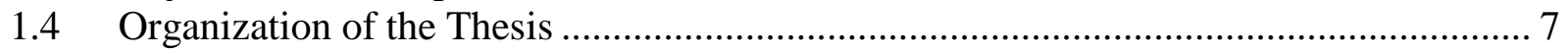

$2 \quad$ Literature Review .................................................8

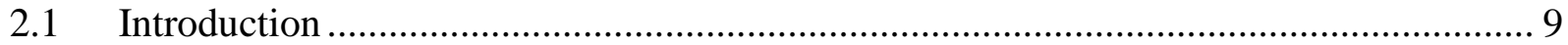

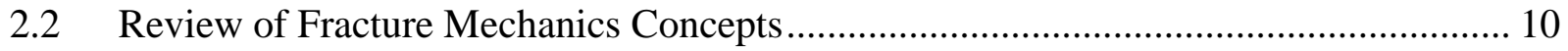

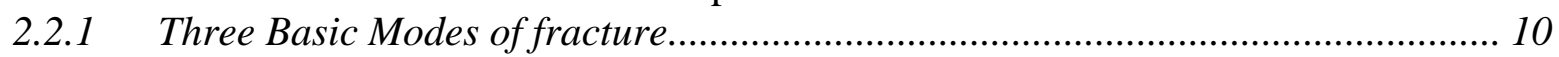

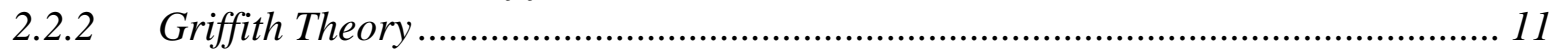

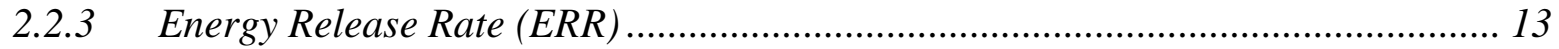

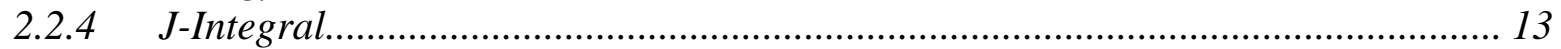

2.2.5 Cohesive Zone Model..................................................................................... 15

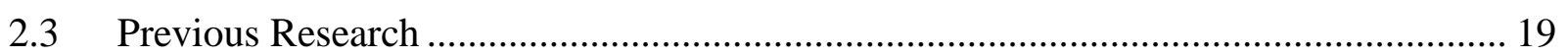

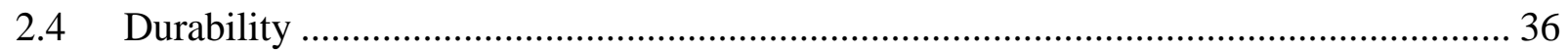

2.4.1 Experimental Case Study (Durability) ......................................................... 39

3 Materials and Testing Program ............................. 45

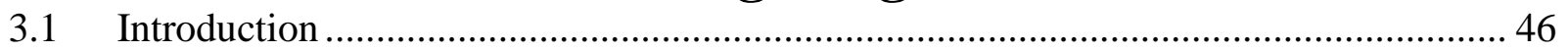

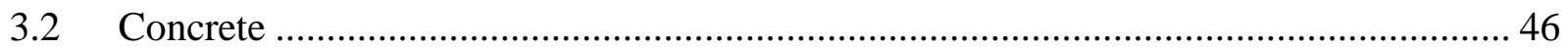

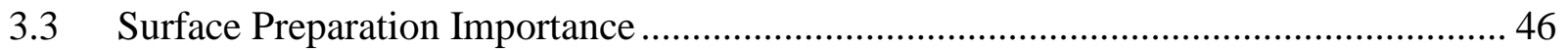

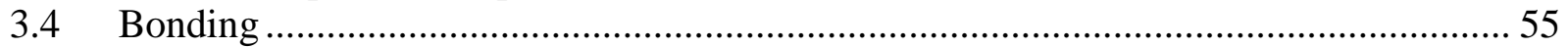

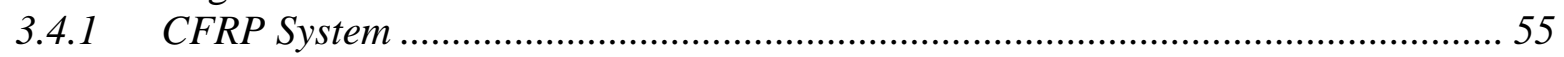

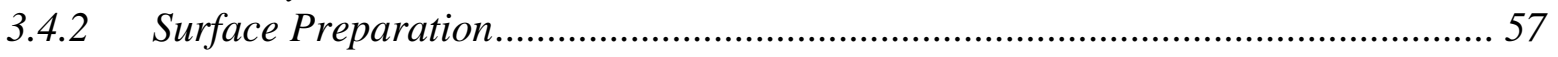

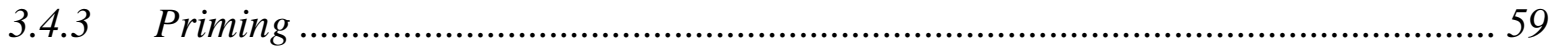

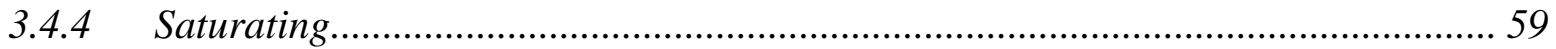

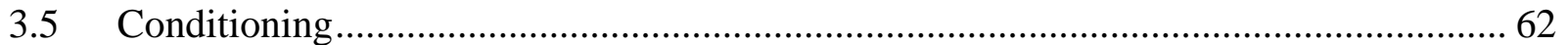

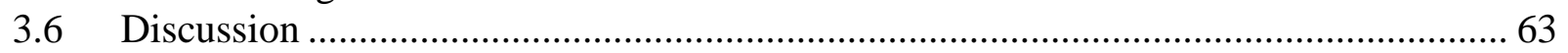

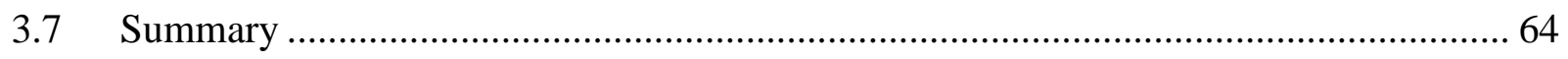

4 Testing Method and Data Reduction..................... 65

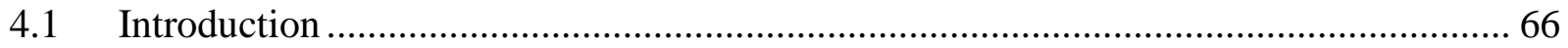

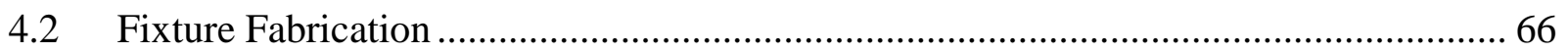

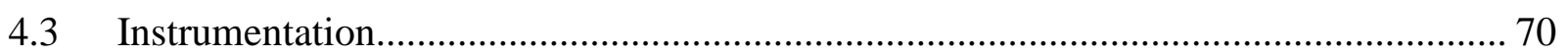

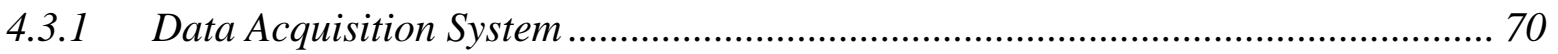

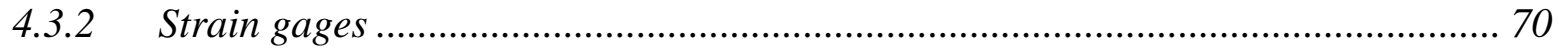

4.3.3 Linear Variable Displacement Transformer (LVDT) ........................................ 71

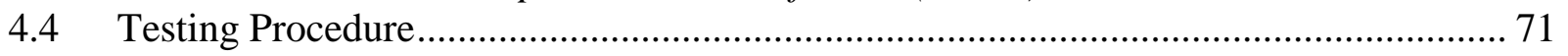

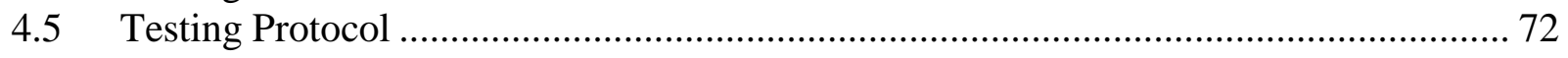

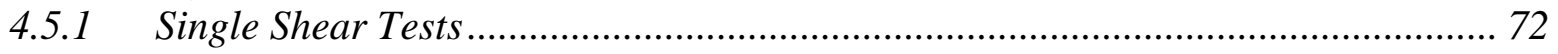

4.5.2 Pull-Off Testing............................................................................................... 73

4.5.3 Obtaining Surface Profile using Coordinate Measuring Machine (CMM)........... 74

4.5.4 Interface Relative Humidity (IRH) Measurement .................................................. 76

4.5.5 Stereo Microscopy ..................................................................................... 77 


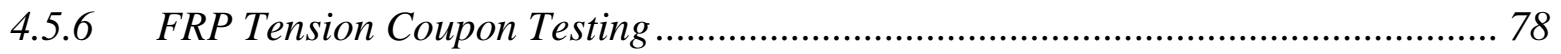

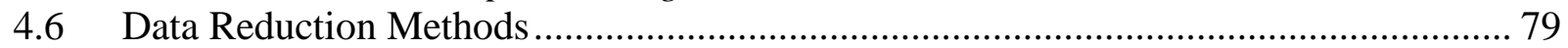

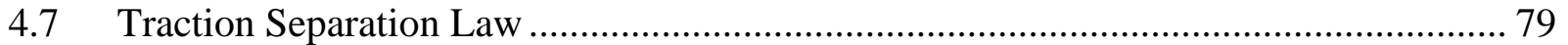

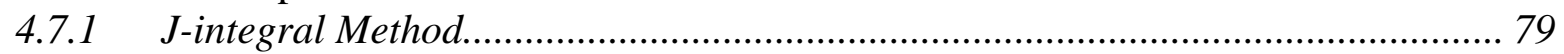

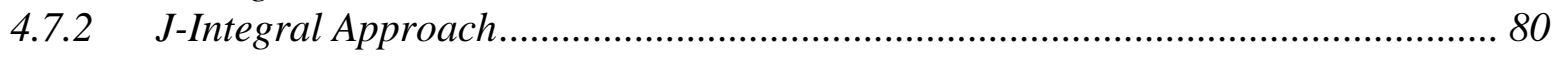

4.7.3 Traditional Method Using Mounted Strain Gages ............................................... 83

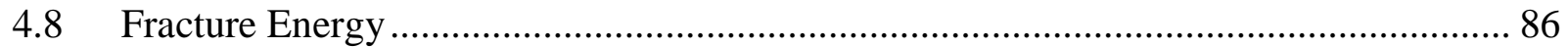

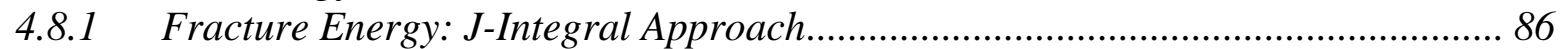

4.8.2 Fracture Energy: Traditional Approach .............................................................. 86

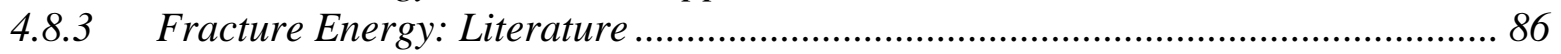

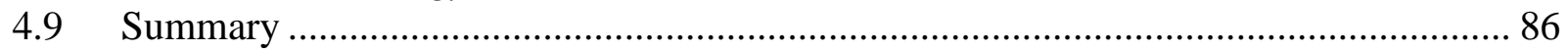

$5 \quad$ Testing Results and Discussion ..............................8 87

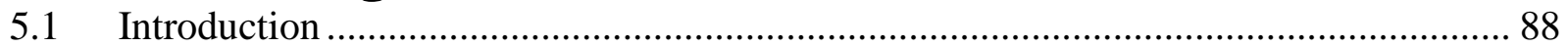

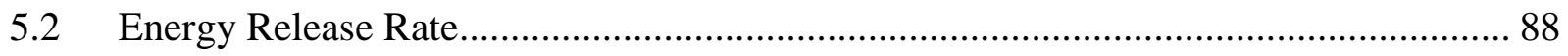

5.3 Traction Separation Law ................................................................................... 90

5.4 J-integral approach ............................................................................................ 90

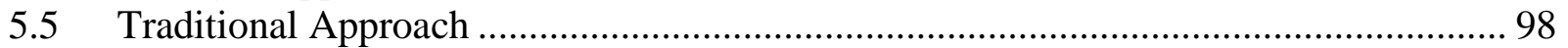

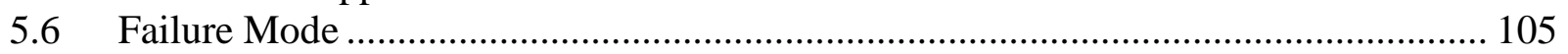

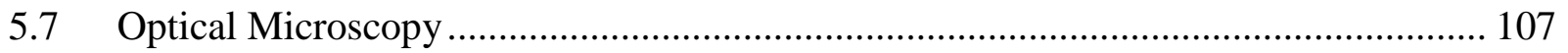

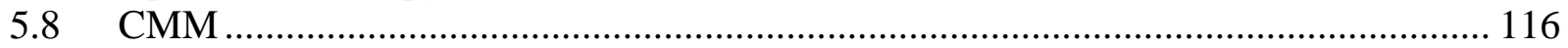

5.9 Interface Relatively Humidity Measurement …………............................................. 122

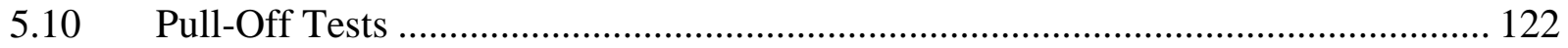

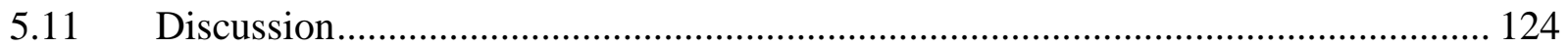

6 Concluding Remarks and Recommendations...... 129

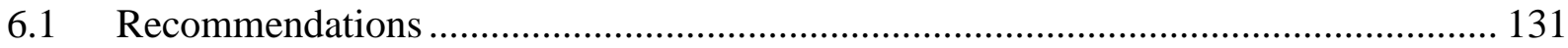

References ................................................................................... 132

Appendices ........................................................................... 140 


\section{List of Figures:}

Figure 1-1 Deficient Bridges all over the US as of December 2009 per FHWA; SD= Structurally

Deficient, FO= Functionally Obsolete 2

Figure 1-2 (a) Single Shear Test, (b) Double Shear Test, (c) Single and Double Shear Test, (d) Three-Point Bending Test. 5

Figure 2-1 Modes of Fracture (a) Mode I, (b) Mode II, (c) Mode III......................................... 11

Figure 2-2 A sharp crack in an Infinite Plate Subjected to Tensile Stress (Adopted from T. L.

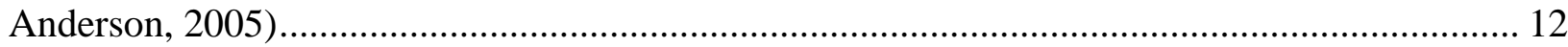

Figure 2-3 A Circular crack in an Infinite Plate Subjected to Tensile Stress (Adopted from T. L.

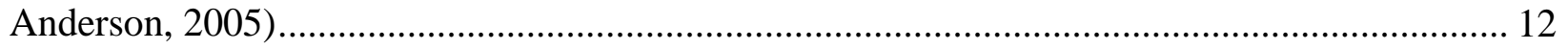

Figure 2-4 Arbitrary Contour around the Crack Tip (Adopted from T. L. Anderson, 2005) ....... 14

Figure 2-5 Arbitrary Contours $\Gamma_{1}$ and $\Gamma_{2}$ around the crack tip connected by $\Gamma_{3}$ and $\Gamma_{4}$ (Adopted

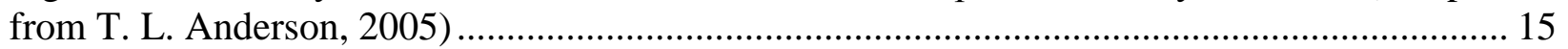

Figure 2-6 Typical Stress-Strain Curve of a Quasi-Brittle Material......................................... 15

Figure 2-7 Fictitious Crack Model (Adopted from Elices et al., 2002) .................................... 17

Figure 2-8 Typical Bilinear Traction-Separation Law (adopted from Camanho PP and Dávila,

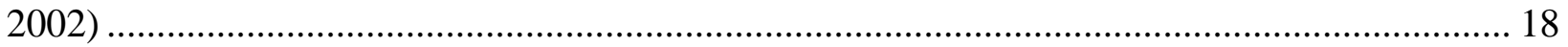

Figure 2-9 Interfacial Failure Modes (adopted from Karbhari et al., 1997 )............................. 21

Figure 2-10 (a) non-symmetric set up (b) symmetric set up (adopted from Taljsten; 1996)........ 22

Figure 2-11 Effective Bond Lenggh ................................................................................ 24

Figure 2-12 Effective Bond Length (adopted from Ali-Ahmad et al., 2007) ............................. 24

Figure 2-13 Pull-apart test (Adopted from Kamel et al. (2006)) ............................................... 25

Figure 2-14 Schematic View of Nakaba et al.'s Model........................................................ 29

Figure 2-15 Linear-Exponential Traction-Separation Law; (a) Mode I, (b) Modes II and III

(Adopted from Wang and Davalos (2004)) ....................................................................... 31

Figure 2-16 Bilinear Mixed Mode Cohesive Law (adopted from Camanho and Dávila (2002)). 31

Figure 2-17 Bilinear damage cohesive law (adopted from Qiao and Chen (2008)) .................... 33

Figure 2-18 Graphical Representation of CZM developed by Högberg (2006) (adopted from

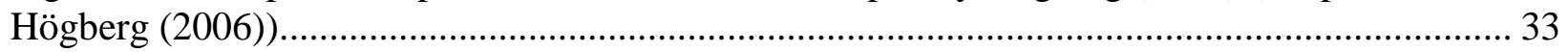

Figure 2-19 Bi-Linear Model (adopted from Yuan et al. (2004))............................................ 34

Figure 2-20 cohesive-bridging model (Adopted from Wang (2007 b)) ................................... 36

Figure 2-21 The overall effect of moisture on composite materials (Nkurunziza et al. ,2005).... 39

Figure 2-22 Setup to measure interface region relative humidity (Adopted from Ouyang and Wan

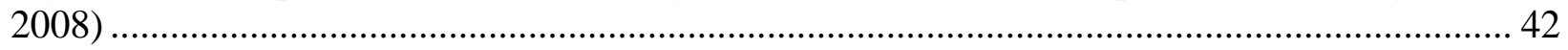

Figure 2-23 Microstructure of bond interface region between FRP and concrete (adapted from

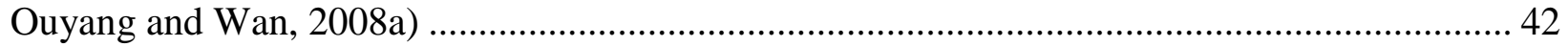

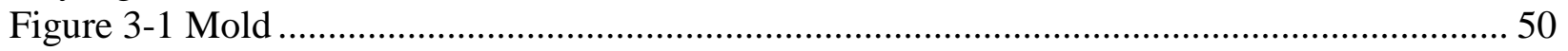

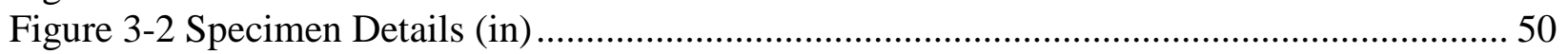

Figure 3-3 Water-Based Mold releasing Agent ............................................................. 52

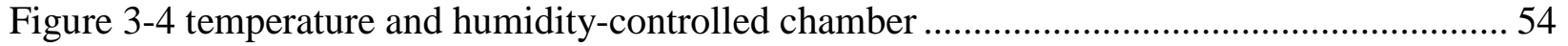

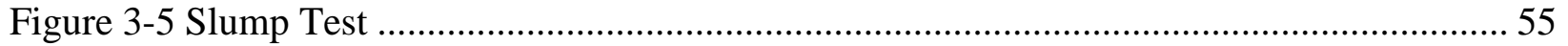

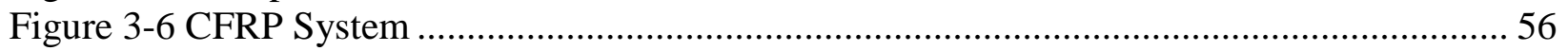

Figure 3-7 (a) Portable Sandblasting, (b) Sandblasting Cabinet.............................................. 58

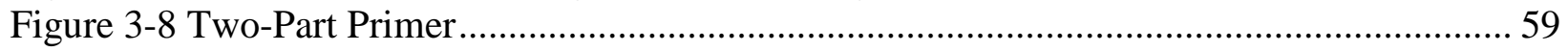

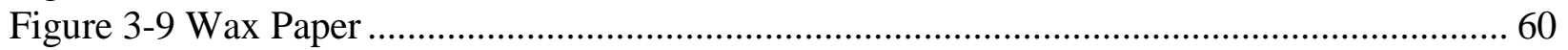




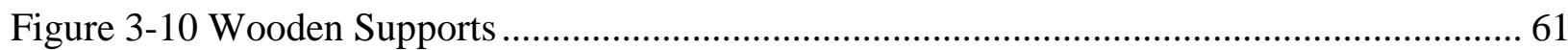

Figure 3-11 (a) Conditioning Tanks, (b) wrapping the rope around the specimens ........................63

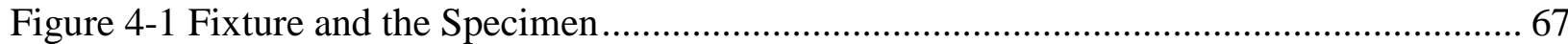

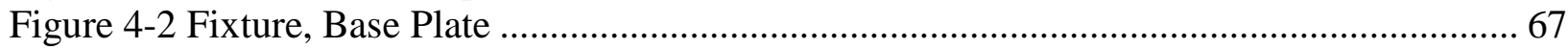

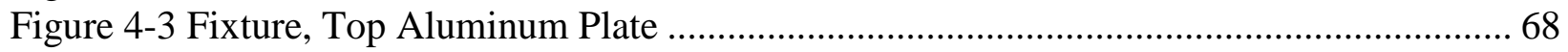

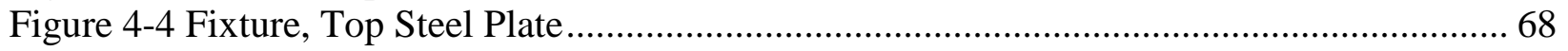

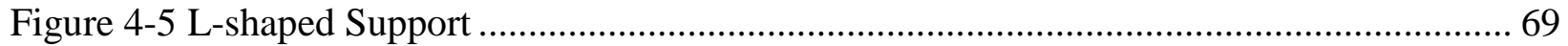

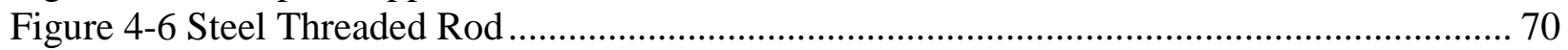

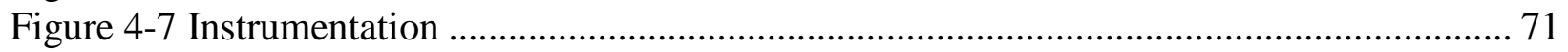

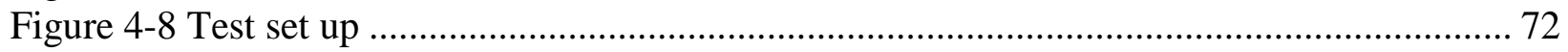

Figure 4-9 Testing Plan (Excluding CMM, and Optical Microscopy) ........................................ 73

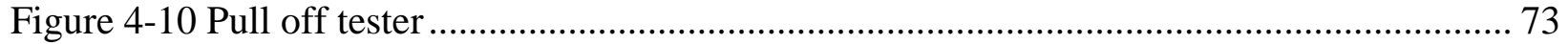

Figure 4-11 (a) Adhesive Putty, (b) Temporay Putty Frame ................................................................ 75

Figure 4-12 (a) CMM, (b) Recording the Coordinates, (c) GEOMET .......................................... 76

Figure 4-13 (a) Humidity measurement, (b) Humitest .................................................................. 76

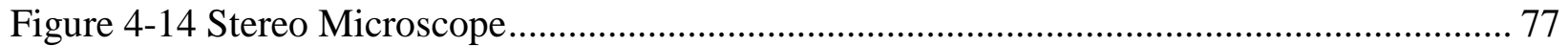

Figure 4-15 Fractured Surface Division for Recording Optical Images........................................ 77

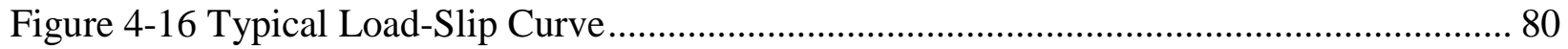

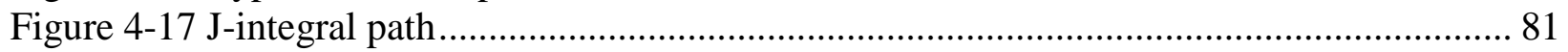

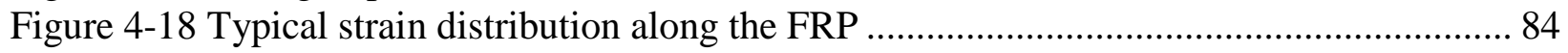

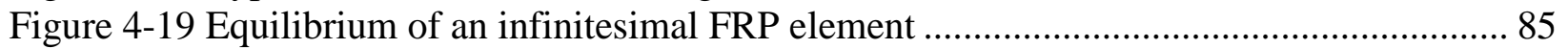

Figure 4-20 (a) Strain Distribution as obtained from Strain gages, (b) average shear stress, (c)

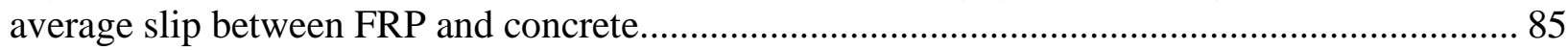

Figure 5-1 Decay of ERR for Different Immersion Durations and Temperatures ........................ 89

Figure 5-2 Decay of ERR for a Given Immersion Period (5, 13, and 15 weeks) ......................... 90

Figure 5-3 Schematic View of a Typical Load-Slip Curve ........................................................... 91

Figure 5-4 Predicted Load for Various Temperatures at Week 11 ……......................................... 92

Figure 5-5 Predicted Load for Various Temperatures at Week 13 …………………….................. 92

Figure 5-6 Predicted Load for Various Temperatures at Week 15 ................................................... 93

Figure 5-7 Experimental vs. Predicted Load Values (T4-W9) .......................................................... 93

Figure 5-8 Traction-Separation Law....................................................................................... 94

Figure 5-9 Traction-Separation Law; (a) T1, (b) T2 .................................................................... 95

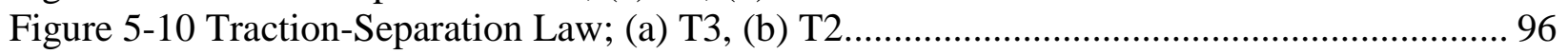

Figure 5-11 $\tau$-s Curves for various Temperatures at the end of Week 11 ....................................... 98

Figure 5-12 Typical strain distribution along the FRP …………............................................... 98

Figure 5-13 Typical Strain, Stress, and Slip Profiles for Different Load Levels............................ 99

Figure 5-14 Traction-Separation Law (J-integral vs. Traditional Approach) ............................... 101

Figure 5-15 Traction-Separation Law (J-integral vs. Traditional Approach) ................................ 102

Figure 5-16 Traction-Separation Law (J-integral vs. Traditional Approach) ................................ 102

Figure 5-17 Traction-Separation Law (J-integral vs. Traditional Approach) ............................... 103

Figure 5-18 Traction-Separation Law (J-integral vs. Traditional Approach) ................................ 103

Figure 5-19 Traction-Separation Law (J-integral vs. Traditional Approach) ................................ 104

Figure 5-20 Traction-Separation Law (J-integral vs. Traditional Approach) ................................ 104

Figure 5-21 Traction-Separation Law (J-integral vs. Traditional Approach) ............................... 105

Figure 5-22 (a) Abrupt Failure, (b) Delaminated FRP, and the Concrete Counterpart, (c) Inappropriate Failure. 


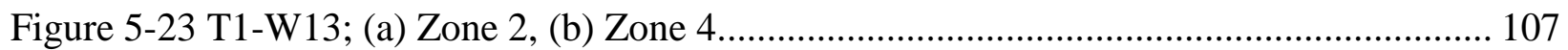
5-24 Relative Fracture Surface upon Images Processing (T1, Weeks 11-13-15, and control)... 108 Figure 5-25 Relative Fracture Surface upon Images Processing (T2, Weeks 11-13-15 and control) 108

Figure 5-26 Relative Fracture Surface upon Images Processing (T3, Weeks 11-13-15 and control) 109

Figure 5-27 Relative Fracture Surface upon Images Processing (T2) ....................................... 110

Figure 5-28 Change of the Fracture Surface after 11 weeks for different tempearaures............. 110

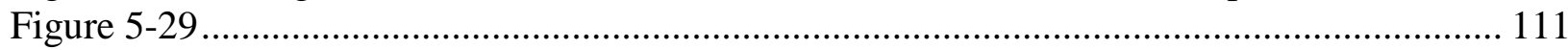

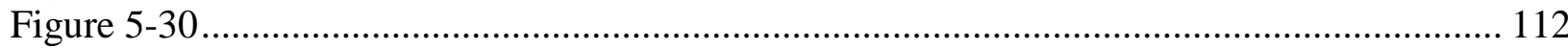

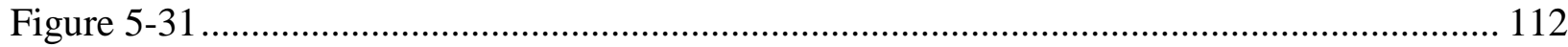

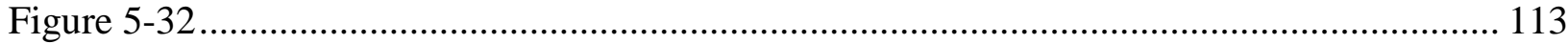

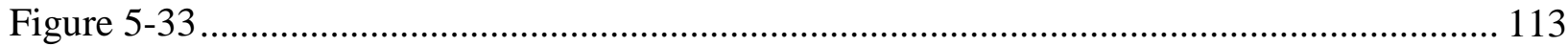

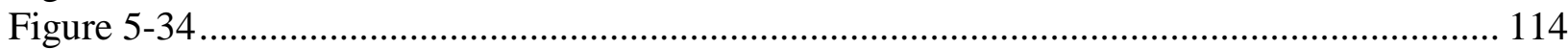

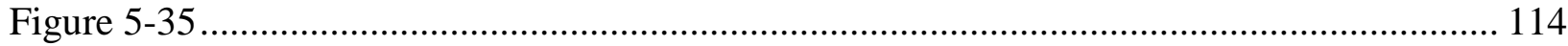

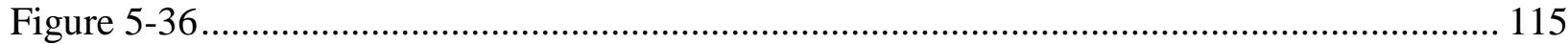

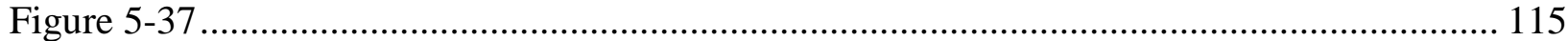

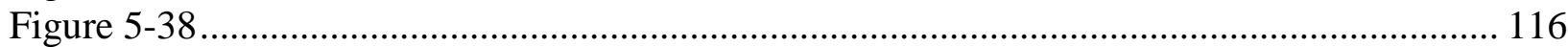

Figure 5-39 Change of Fracture Area with Temperature................................................... 117

Figure 5-40 Change of Fracture Area with Immersion Duration ......................................... 117

Figure 5-41 Change of Fracture Area with Immersion Duration (T2)................................... 118

Figure 5-42 T1 Contours; (a) W11, (b) W13, (c) W15........................................................ 119

Figure 5-43 T2 Contours; (a) W3, (b) W5, (c) W7, (d) 9, (e) W11, (f) W13, (g) W15............. 120

Figure 5-44 T3 Contours; (a) W11, (b) W13, (c) W15........................................................ 121

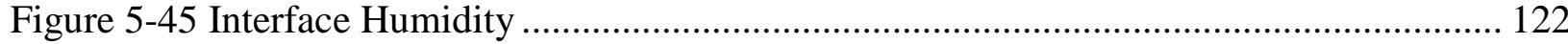

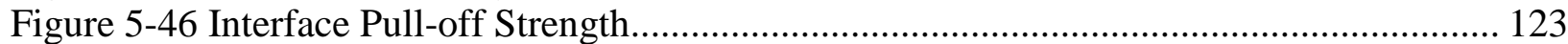

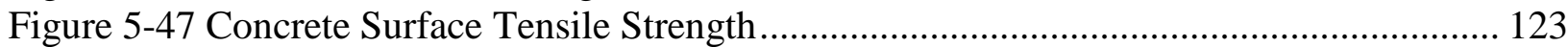

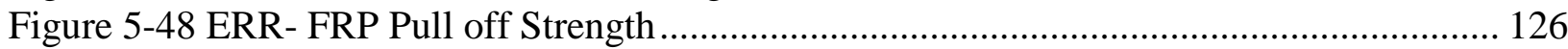

Figure 5-49 ERR-Concrete Surface Tensile Strength...................................................... 127

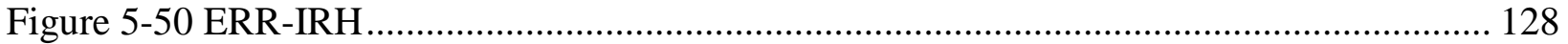




\section{Introduction}




\subsection{Introduction}

In December 2009, annual Federal Highway Administration (FHWA) statistics revealed that about $12 \%$ of all bridges in the US are structurally deficient, which amounts to $31,200,000 \mathrm{mi}^{2}$. In West Virginia alone, 15\% of bridges are categorized as being structurally deficient. Based upon FHWA classifications, a structurally deficient bridge means one that is either in poor structural condition due to deterioration and/or damage, or its waterway opening is extremely insufficient and causes intolerable traffic interruptions.

FHWA statistics further reveal that about $13 \%$ of the US bridges are functionally obsolete, and that $21 \%$ of WV bridges are also functionally obsolete. The FHWA defines a functionally obsolete bridge as one where the traffic demand and geometry guidelines change from those to which the bridge was originally designed to meet. Obviously, structurally deficient bridges are those which have to be attended to immediately (See Figure 1-1).

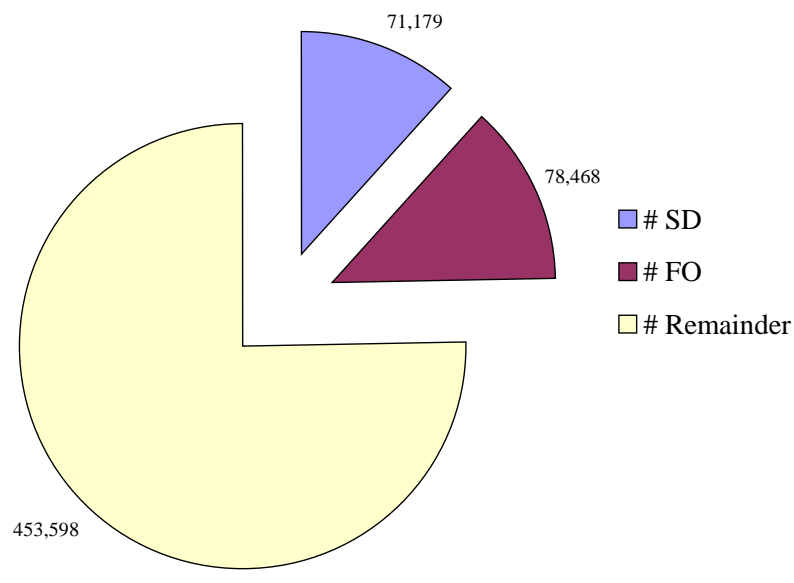

Figure 1-1 Deficient Bridges all over the US as of December 2009 per FHWA; SD= Structurally Deficient, FO= Functionally Obsolete

Degradation of civil infrastructures is not limited to the United States; rather it presents a major challenge throughout the world. As the deterioration of civil infrastructures, especially bridges, continues worldwide, and the replacement of the entire structures cannot be justified financially, the need to develop effective methods to rehabilitate and retrofit these structures becomes more important.

One of the methods traditionally used to repair a deficient bridges for the 30 years is to reinforce the RC beams by bonding a steel plate onto its tension side. Although by applying this 
method, the total replacement of several bridges has been avoided, this method has its own disadvantages, among which the most significant is steel corrosion, which can be accelerated in acidic environments produced by deicing salts. The resulting rust will adversely affect the quality of the plate-concrete bond. Notwithstanding the fact that bonding heavy steel plates to the beam necessitates an experienced, and specialized workforce, and use of the special equipment at the bridge site and, which will increase the overall cost of the operation.

Given these facts, in the past two decades, the use of Fiber Reinforced Polymer (FRP) composites instead of steel plates has been steadily gaining popularity in rehabilitating and retrofitting concrete structures, especially bridges. This is due to of their many advantages, such as ease and speed of construction, resistance to corrosion, low cost of application and maintenance, and high strength-to-weight ratio. One of the most appealing features of composite materials is that unlike the steel plates, they are very light and shipping and storage is much easier. Moreover, as they come in the sheets form, in the event of application in external reinforcement, FRP composites can be easily formed to be bonded to different structures; i.e., the very same batch of materials can be used to reinforce a large variety of structures including beams, columns, bridge decks and so forth, which significantly reduces peripheral expenses.

Despite the fact that the results of short term tests show the effectiveness of this method, the long term performance of externally FRP-bonded structures, or more accurately, the FRPconcrete durability, is still open for debate.

Although there are different failure possibilities for monolithic structures, such as excessive elastic or permanent deformations or fracture, the dominant failure mode of interface in adhesive joint structures is fracture (Boyajian, 2002) . Unlike the structure which can be scaled to different sizes, the interface is not scalable and makes only a small fraction of the entire structure. As a matter of fact, it is the long term performance of the interface which defines the service life of an adhesive joint, such as CFRP-bonded concrete beam, and the effectiveness of this rehabilitation method.

Externally bonded FRP laminates used for either shear or flexural strengthening of the structure are dependent on the shear stress transfer at the concrete-FRP interface. This illustrates the importance of obtaining a good understanding of interface failure under Mode II (shear) loading. 
As explained by Boyajian (2002), in cases dealing with inhomogeneous materials, especially where the area under investigation is composed of a thin layer of two adjacent materials (one with high and the other with a low modulus of elasticity), just like FRP-concrete interface, the traditional fracture mechanics approach by defining the stress intensity factor, $K$, and fracture toughness, $K_{c}$, which is the critical value of $K$, does not work to model the interface. Therefore, instead of the strength criterion, fracture toughness should be defined in terms of energy in order to define the behavior of heterogeneous materials such as FRP-concrete interface.

This is why the energy release rate, $G$, as to be explained in section 2.2.3, is defined as the energy required for extending a crack by a unit area as an alternative to stress intensity factor based on energy approach. Its critical value, $G_{c}$, is a material constant which is an important parameter for designing the adhesive joints.

As explained in 2.2.1, there are three modes of fracture, among which the second mode, Mode II or in-plane-shear Mode is the most important, while dealing with the adhesive joints. Its corresponding fracture toughness, $G_{I I C}$, can be used as a failure criterion.

Since as the shear failure is responsible for failure in a majority of CFRP-bonded concrete beams, the use of $G_{I C}$ appears to be too conservative, because $G_{I C}$ is the least in fracture toughness among the three modes.

Traditionally, there are four testing setups which are the most popular for studying the FRPconcrete interface: (1) flexural three-point bending test; (2) flexural four-point bending test; (3) single shear test; and (4) double shear test. The bending tests utilized for studying Mode I fracture, while the shear tests are for Mode II fractures (See Figure 1-2). 
(a)

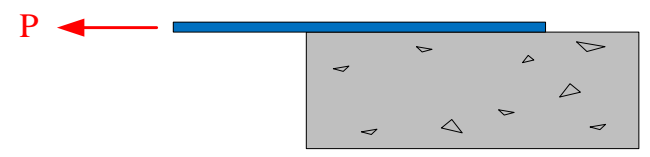

(b)

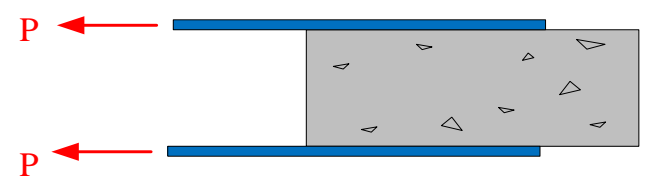

(c)

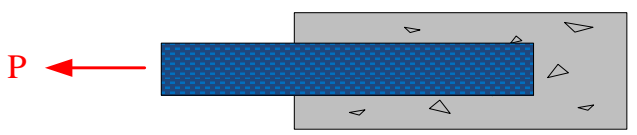

(d)

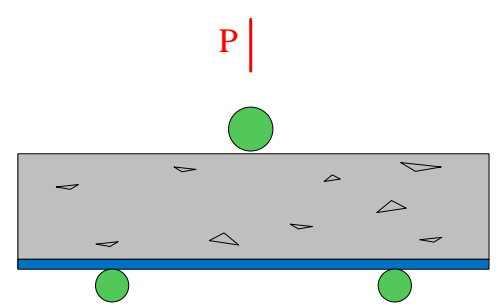

Figure 1-2 (a) Single Shear Test, (b) Double Shear Test, (c) Single and Double Shear Test, (d) Three-Point Bending Test

The single shear test is shown to provide a more realistic representation of shear failure in CFRP- bonded concrete beams, when compared to a double-shear test setup, as the latter is affected by geometric issues to a greater extent (Subramaniam et al., 2007).

In a typical single-shear test, an axial force is applied to the FRP laminate bonded to the concrete substrate, while the concrete is restrained against movement. Using this test setup, the CFRP-concrete interface is subjected to shear stress, thus manifesting a pure Mode II loading. Although Suo and Hutchinson (1990) showed that any interface fracture is actually mixed-mode, Yuan et al. (2004) explained that if it is assumed that both concrete and FRP are only subjected to axial forces and any bending effect is neglected, the debonding would be dominated by a Mode II fracture. This simplifying assumption can be fulfilled by designing the specimen such that the concrete prism is much larger than the FRP laminate, and also by paying special attention to fixture design so as to minimize bending effects.

Qiao et al. (2003) designed a linear, tapered end-notched flexure (TENF) specimen to experimentally measure the Mode II fracture energy of wood-wood and wood-FRP interfaces. This specimen can be used efficiently to evaluate the bi-material interface behaviors under Mode II loading, provided that the two adherents are good in tension. This specimen, however, cannot be used for a concrete substrate because concrete is a tension weak material and will fail under 
tension before fracture occurs, leaving the traditional single or double shear test specimens as the best experimental setups to study the Mode II failure of FRP-concrete.

In this study, a single-shear test is used.

\subsection{Significance}

Despite extensive studies regarding interface fractures under shear loading ( $\mathrm{Xu}$ and Needleman, 1993; Nakaba et al., 2001; Högberg, 2006; Wang, 2006; Ferracuti, 2006; and Wang, 2007), and the durability studies on the composite materials, surprisingly, there are only a few studies found that focus on durability issues in relation to interfacial Mode II fractures. Prior to this research, there was no research on the effect of the two environmental conditions, i.e., immersing in deionized water combined with controlled temperatures on the FRP-concrete Mode II characteristics.

Moreover, in this research, a fracture mechanics method based on the J-integral approach is proposed to evaluate the CFRP-concrete interfacial behavior, which only requires the measurement of the relative slip at the tip of the notch and the corresponding load, whereby the effort of bonding strain gages for the traditional method can be omitted. This also avoids the inherent assumption of linear elastic fracture mechanics (LEFM) behavior, as it is valid only when the size of the deformation zone is small when compared to the crack size, which is not the case for all concrete and even some composite materials.

\subsection{Objectives and Scopes}

In response to the needs mentioned above, i.e., the need to develop a non-strength based model for the FRP-concrete interface which considers durability issues, the objectives of this study are: (1) to develop a Fracture Mechanics Model to characterize the FRP-concrete interfacial behavior under Mode II loading; and (2) to extend the aforementioned fracture mechanics model to study the durability of FRP reinforcing technique in the event of exposure to simulated environmental conditions.

The following simultaneous environmental effects are considered as exposure conditions: (1) immersion in deionized water varying from 0 to 15 weeks; and (2) controlled temperatures varying from $25^{\circ} \mathrm{C}$ to $60^{\circ} \mathrm{C}\left(77^{\circ} \mathrm{F}\right.$ to $\left.140^{\circ} \mathrm{F}\right)$ of samples while immersed in water. 
Among different types of FRP composites, carbon and glass fibers are the most popular composites in civil engineering applications. With respect to repair and retrofit of civil engineering infrastructures, especially bridges, however, carbon fibers are most commonly used, due to their higher resistance to environmental attack. Therefore, carbon FRP is chosen to be used in this research. As for the concrete, since concrete material used for most bridges is normal concrete as opposed to High Performance Concrete, only normal concrete with Type I cement is used throughout the entirety of this research, and the specimens are tested in single shear test setup.

The parameters to be obtained and measured are the Mode II critical strain energy release rates, $G_{I I C}$, the maximum load, and the corresponding slip. Additional parameters to be studied are strains in the fiber direction of the FRP laminate and concrete surface tensile strength. Some representative delaminated FRP laminates are tested in tension to see whether there is any loss of FRP mechanical properties due to conditioning. Stereo microscope is used to record optical images of the fracture surface on some representative delaminated FRP and the images are analyzed using excel. Coordinate Measuring Machine (CMM) was used to obtain a 3-D surface profile of the same laminates. The change of the ions of the water in which the samples were held was monitored.

\subsection{Organization of the Thesis}

Chapter 2 begins with a brief review of basic concepts of fracture mechanics and durability which will be used throughout this thesis afterwards. Then, the most prominent relevant studies on developing a constitutive law for FRP-concrete interface are reviewed along with studies addressing the durability issues. Next, testing program and materials are explained in Chapter 3. Chapter 4 discusses the testing method, and data reduction methods. Finally, the results are presented and discussed in chapter 5 . 
2 Literature Review 


\subsection{Introduction}

Despite the fact that composite materials have been around for decades and have been used with a high level of confidence in the automotive, marine, and aerospace industries for so long, and although there are well-documented and well-accepted design codes for the application of FRP materials in these industries, there is no such design code available for FRP application in civil infrastructure applications. While various guidelines and reports have been published by ACI and NCHRP regarding FRP application for repairing and retrofitting highway bridges (ACI 440.2R, and NCHRP Report 514, respectively) none are currently utilized as a determining standard.

While there are varying failure possibilities for monolithic structures, which include elastic or permanent deformations or fracture, fractures are the dominant failure mode of interface in adhesive joint structures (Boyajian, 2002). Unlike a structure which can be scaled to different sizes, the interface is not scalable and makes for only a small fraction of the entire structure. Therefore, the quality of the interface and the interfacial fracture energy is the most critical parameter in defining the service life of an adhesive joint such as a CFRP-bonded concrete beam.

There are several models based on the fracture mechanics approach which are used to describe concrete behavior, including the Cohesive Zone Model (CZM), (aka fictitious crack model or traction-separation law), introduced by Hillerborg. This is the most popularly utilized model. CZM is discussed in more details in section 2.2.5.

FRP reinforced concrete structures in general, and bridges in particular, are exposed to a plethora of environmental attacks and extreme changes in weather and manmade variables during their service life. This includes wet and dry cycles, freezing and thawing conditions, temperature variations, de-icing salts, etc. These attacks weaken the FRP-concrete bond and decrease its load-carrying capacity. It can be deduced that most of the environmental attacks listed are somehow related to varying moisture levels. Therefore, the study of the moisture effect on the bond between FRP and concrete is important for concrete structures externally reinforced by FRP laminates, as it can help solve durability problems.

When it comes to durability regarding FRP application for rehabilitation and retrofit purposes, there are two main issues which come to mind: (1) the durability of FRP, and (2) the durability of the FRP-concrete interface. 
Since carbon fibers tend to be the industry standard to rehabilitate and retrofit the bridges, there is no concern regarding the FRP durability itself, as carbon fibers are not affected by humidity or the alkalinity of the environment. Therefore, the focus of this chapter will be on durability of the interface.

In this chapter, first, the concepts and definitions of fracture mechanics and durability considerations are reviewed briefly. Then, in section 2.3, the most prominent relevant studies and efforts to develop the FRP-concrete traction-separation law are reviewed.

\subsection{Review of Fracture Mechanics Concepts}

\subsubsection{Three Basic Modes of fracture}

Although crack propagation can occur in different directions and patterns, there are only three different loading scenarios. These are denoted as Mode I, Mode II, and Mode III, whereby a crack can occur (See Figure 2-1). Any arbitrary loading on a cracked body can be represented as a linear combination of these three basic modes; in other words, the crack tip deformation and its corresponding stress field in any arbitrary loading can be obtained by superposition of these three modes. In Mode I (opening mode), the load is applied normal to the crack plane, which tends open the crack. In Mode II, which is also called in-plane shear mode, the two crack faces are forced to slide with respect to one another. Mode III (tear Mode) corresponds to out-of-plane shear. (T. L. Anderson, p.43, 2005).

Although Mode I characteristics are of great importance since they make the lower limit of the fracture toughness for that material in a given testing condition, Mode II failures play a more dominant role when it comes to rehabilitating or retrofitting the concrete structures. By externally bonding FRP plates or laminates, this is especially true for bridges. The main mode of failure in FRP-bonded concrete beams is delamination or separation of the FRP layer from the concrete substrate. Such failure is dominated by the FRP-concrete interface properties, among which the most important is Mode II fracture toughness. Delamination of FRP layers externally

bonded to concrete beams is very brittle, and the failure is rather sudden and catastrophic, since it occurs without warning. Thus, in order to make a more reliable design, obtaining a more comprehensive understanding of FRP-concrete interface behavior is necessary. 


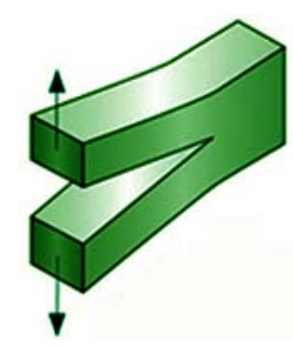

(a)

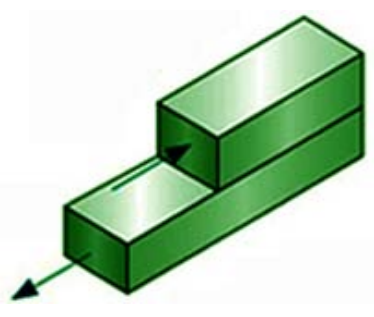

(b)

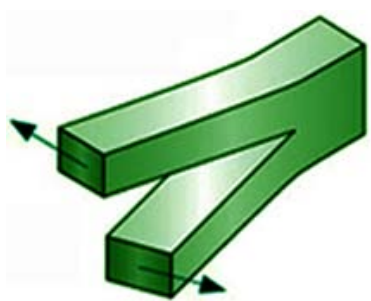

(c)

Figure 2-1 Modes of Fracture (a) Mode I, (b) Mode II, (c) Mode III

\subsubsection{Griffith Theory}

Fracture Mechanics in its present form began with Alan Arnold Griffith’s theory of energy balance (1893-1963). In 1920, he applied the first law of thermodynamics to the formation of a crack and explained that crack propagation is basically governed by the energy balance: (Griffith, 1920):

"It may be supposed, for the present purpose, that the crack is formed by the sudden annihilation of the tractions acting on its surface. At the instant following this operation, the strains, and therefore, the potential energy under consideration, have their original values; but in general, the new state is not of the equilibrium. If it is not a state of equilibrium, then by the theorem of minimum potential energy, the potential energy is reduced by attainment of equilibrium; if it is a state of equilibrium, the energy does not change.”

He concluded that a crack can form or an existing crack can propagate only when this process can cause the total energy to decrease or remain constant. Therefore, when crack growth occurs under a constant total energy, (i.e., in equilibrium condition) that specific point can be considered to be the critical state for the fracture to occur (T. L. Anderson, p.29, 2005).

Griffith’s energy balance theory can be expressed as follows:

$\frac{d E}{d A}=\frac{d \Pi}{d A}+\frac{d W_{s}}{d A}=0$

or

$\frac{d \Pi}{d A}=-\frac{d W_{s}}{d A}$

where:

$d A=$ the incremental increase in the crack area

$E \quad=$ total energy

$\Pi=$ potential energy

$W_{s}=$ work required to create new crack surfaces 
According to his theory, the fracture can only occur if the energy stored in the structure is sufficient to overcome the surface energy of the newly created crack faces.

For an infinite plate containing a sharp crack subjected to tensile stress (Figure 2-2), which also, denotes the plane stress state, the above equation will be simplified as follows to give the fracture stress:

$\sigma_{f}=\left(\frac{2 E \gamma_{s}}{\pi a}\right)^{1 / 2}$

where $\gamma_{s}$ is the surface energy of the material.

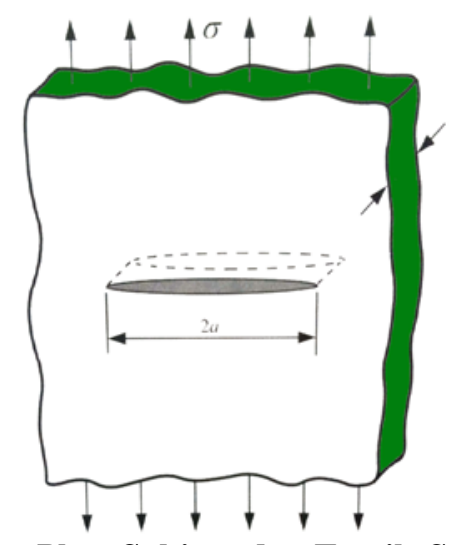

Figure 2-2 A sharp crack in an Infinite Plate Subjected to Tensile Stress (Adopted from T. L. Anderson, 2005)

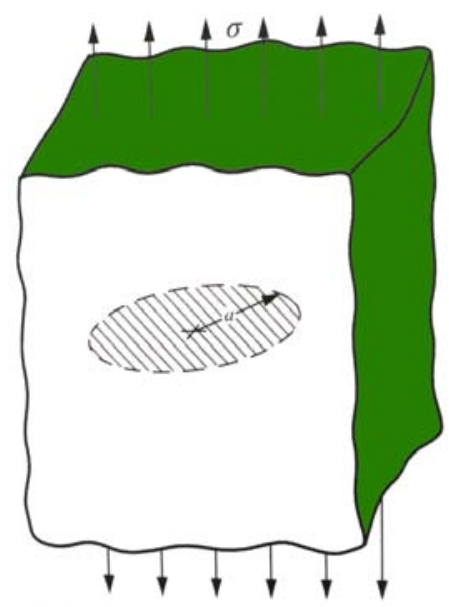

Figure 2-3 A Circular crack in an Infinite Plate Subjected to Tensile Stress (Adopted from T. L. Anderson, 2005) 
By the same token, for an infinite plate subjected to tensile stress, (Figure 2-3), containing a circular crack and denoting the plane strain state, the Equation (2-2) will be simplified as follows to give the fracture stress:

$$
\sigma_{f}=\left(\frac{2 E \gamma_{s}}{\pi\left(1-v^{2}\right) a}\right)^{1 / 2}
$$

where $v$ is the Poisson's ratio, and $E$ is the modulus of elasticity (T. L. Anderson, pp.29-30, 2005).

\subsubsection{Energy Release Rate (ERR)}

An alternative approach to the Griffith theory which was more practical for solving engineering problems was introduced by Irwin in 1956. He defined the concept of Energy Release Rate, $G$, which is the energy the energy required to extend a preexisting crack by an infinitesimal unit of area:

$$
G=-\frac{d \Pi}{d A}
$$

It should be noted that the term rate does not refer to derivative with respect to time; however, it refers to the rate of change in potential energy with respect to the crack area (T. L. Anderson, p.35, 2005). The ERR for the plane stress and plane strain states as shown in Figure 2-2 and Figure 2-3 are as follows:

$$
\begin{array}{ll}
G=\frac{\pi \sigma^{2} a}{E} & \text { Plane Stress } \\
G=\frac{\pi \sigma^{2} a\left(1-v^{2}\right)}{E} & \text { Plane Strain }
\end{array}
$$

Crack growth occurs when the ERR reaches a critical value called Fracture Toughness, $G_{C}$ which is a very important material property. The fracture toughness of each fracture mode is denoted by $G_{c}$ in honor of Griffith and the subscript of I, II, or III, i.e., $G_{I c}, G_{I I c}, G_{I I I c}$.

\subsubsection{J-Integral}

Rice (1968) introduced a novel concept called the J-integral which has helped extend the horizon of Fracture Mechanics beyond the limits of LEFM. By idealizing the Elastic-Plastic deformation to a Nonlinear Elastic one, Rice showed that the nonlinear energy release rate, $J$, can be written as a path-independent line integral (T. L. Anderson, p.108, 2005). 
Considering an arbitrary counterclockwise path $(\Gamma)$ surrounding a given crack tip as shown in Figure 2-4, Rice defined the $\mathrm{J}$ integral as follows:

$$
\begin{aligned}
& J=\oint_{\Gamma}\left(w d z-\vec{T} \cdot \frac{\partial \vec{u}}{\partial x} d s\right) \\
& w=w_{(\varepsilon)}=\int_{0}^{\varepsilon} \sigma_{i j} d \varepsilon_{i j}
\end{aligned}
$$

where:

$$
\begin{array}{ll}
w & =\text { strain energy } \\
\vec{u} & =\text { displacement vector } \\
\vec{T} & =\text { traction vector acting outward normal to } \Gamma \text { defined as } T_{i}=\sigma_{i j} n_{j} \\
\sigma_{i j} & =\text { components of stress tensors } \\
\varepsilon_{i j} & =\text { components of strain tensors } \\
n_{j} & =\text { components of the unit vector normal to } \Gamma
\end{array}
$$

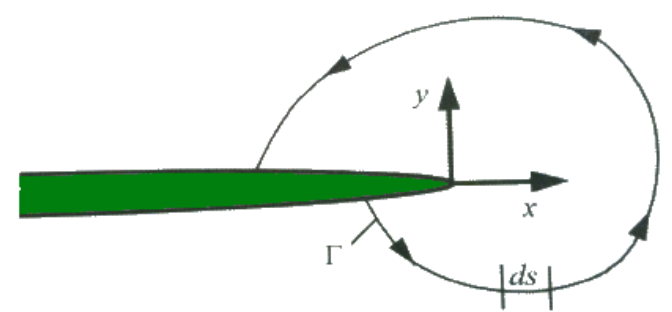

Figure 2-4 Arbitrary Contour around the Crack Tip (Adopted from T. L. Anderson, 2005)

For a two dimensional cracked body, Rice proved that the J-integral is equal to the energy release rate for a linear or nonlinear elastic material.

Rice showed that $J$ is independent of the path chosen around the crack tip. This is why Jintegral is referred to as being path-independent.

Figure 2-5 shows two arbitrary contours surrounding a crack tip. These contours are connected through two other contours, $\Gamma_{3}$ and $\Gamma_{4}$, along the crack face, thus creating a close path around the crack tip. The J-integral on the closed path is zero, and equals the contribution from each contour (T. L. Anderson, p.157, 2005): 
$J=J_{1}+J_{2}+J_{3}+J_{4}=0$

However, on $\Gamma_{3}$ and $\Gamma_{4}$ segments, there is no traction force making $\mathrm{J}_{3}=\mathrm{J}_{4}=0$. Therefore, $\mathrm{J}_{1}=-$ $\mathrm{J}_{2}$. The reason behind the negative sign is that $\Gamma_{1}$ is counterclockwise, while $\Gamma_{4}$ is clockwise. Therefore, any arbitrary path around the crack tip with the same direction will yield the same value for J-integral.

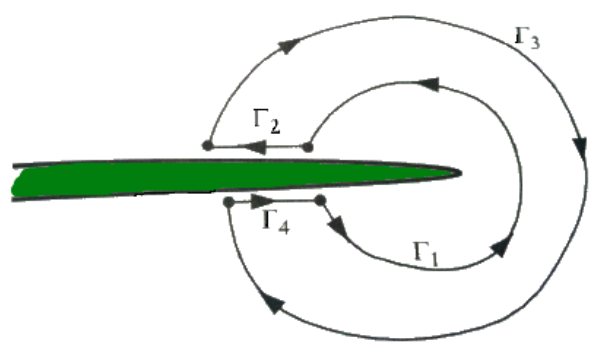

Figure 2-5 Arbitrary Contours $\Gamma_{1}$ and $\Gamma_{2}$ around the crack tip connected by $\Gamma_{3}$ and $\Gamma_{4}$ (Adopted from $T$. $L$. Anderson, 2005)

\subsubsection{Cohesive Zone Model}

Although concrete is usually assumed to be a brittle material, it is actually quasi-brittle, i.e., after the peak stress, the slope of the stress-strain curve decreases, and therefore shows a softening behavior (Figure 2-6).

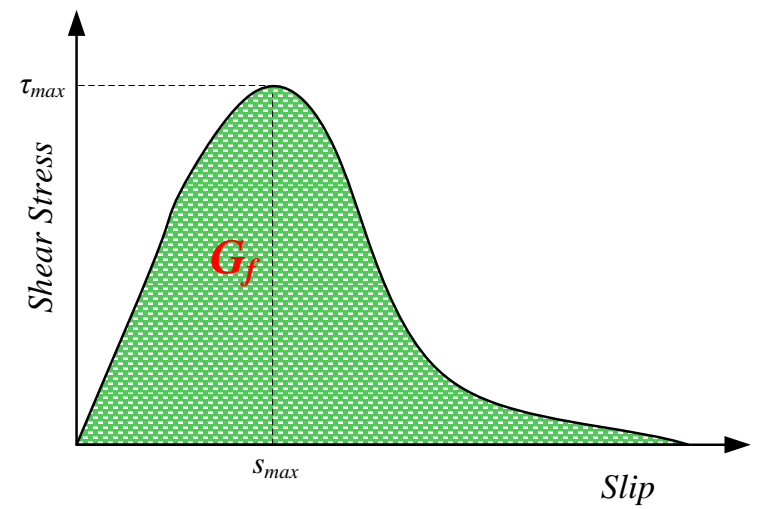

Figure 2-6 Typical Stress-Strain Curve of a Quasi-Brittle Material

This softening effect is due to formation of microcracks in the area in the vicinity of the crack where the energy dissipation occurs. This is called the Fracture Process Zone (FPZ). The bulk material property outside FPZ is linear elastic. In quasi-brittle materials, the size of the FPZ compared to the crack length and the size of the specimen is relatively large, which violates the inherent assumption of linear elastic fracture mechanics (LEFM) behavior, as it is only valid when the size of the deformation zone is small compared to the crack size. 
The "Cohesive Zone Model”, pioneered by Dugdale and Barenblatt regards fracture as a gradual phenomenon, in which separation takes place across an extended crack tip, or cohesive zone, and is resisted by cohesive tractions” (Ortiz and Pandolfi, 1999). The crack extends while simultaneously transferring the stress from one face to the other. Barenblatt showed that CZM can remove the singularity at the crack tip as predicted by LEFM.

Högberg defines CZM as follows: "cohesive law describes the activities in the cohesive zone in terms of the traction and the separation of the surfaces to be formed under the fracture process.” (J, L. Högberg, 2006)

Over the course of the past three decades, cohesive zone models have been used to describe the nonlinear response in the vicinity of the crack tip, due to their simplicity. A variety of materials have been analyzed using those models; namely, metals, polymers, ceramics, and interfaces.

As for CZM application in the study of interfaces, Needleman (1987) has found them to be especially useful with two adjoining substrates, which are stronger compared to the interface, as is the case for CFRP-concrete interface. These models are particularly of interest in numerical modeling of materials, which show the softening behavior. Cohesive Zone Models are usually implemented in FEM packages to simulate the crack propagation using interface elements along a potential crack. Therefore, once the path of the crack growth is known in advance, as in the case of FRP-bonded concrete beams, using the CZM concept sounds promising, as it avoids any remeshing at each load step during the crack growth in order to provide a fine mesh at the crack tip.

An appealing feature of these models is that they do not presume any particular type of constitutive response in the bulk of the material, and successive crack growth is a natural outcome of the analyses.

Hillerborg et al. (1976) were the first to use CZM for concrete. This model, called Fictitious Crack Model, can be used for both cracked and uncracked bodies, providing that a crack initiation is defined.

The CZM connects strength-based analysis of structures to the energy-based fracture mechanics approach. Here, crack initiation is analyzed using strength-based criteria, and its propagation is mostly analyzed through fracture mechanics approach considering fracture 
energy. Thus, Cohesive Zone Models are capable of predicting both the onset and the propagation of crack (Camanho PP and Dávila, 2002).

It has been also shown that as long as the area under the traction-relative displacement curve is equal to the fracture energy, irrespective of its shape, the traction-separation law can well predict the interface behavior. This is yet another benefit of working with these models.
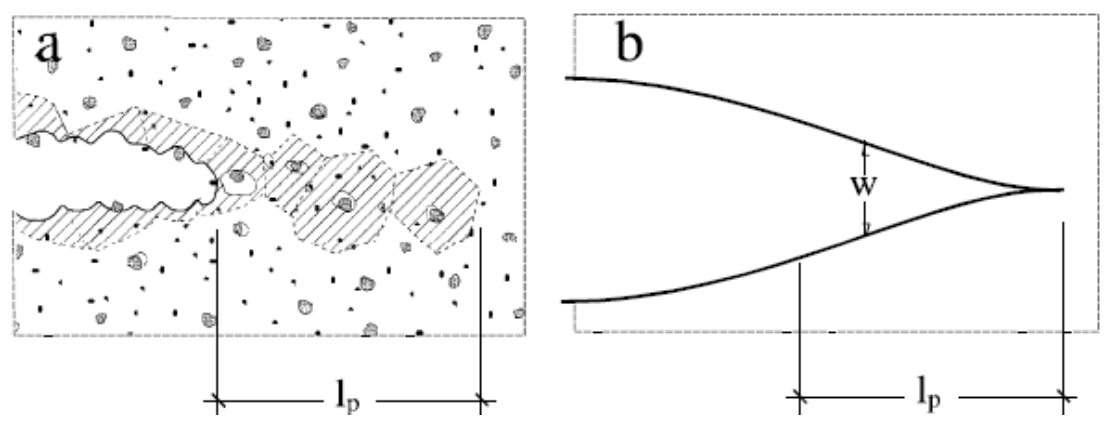

Figure 2-7 Fictitious Crack Model (Adopted from Elices et al., 2002)

It is assumed that crack propagation begins when the stress at the crack tip equals the ultimate strength of the material. During crack propagation, energy dissipation occurs only in the FPZ while the material away from this zone behaves elastically.

As shown in Figure 2-7, two different crack tips are assumed for a fictitious crack: Real Crack Tip (RCT), and Fictitious Crack Tip (FCT). RCT is the point between the real crack and the FPZ, while the FCT is the point between the FPZ and the uncracked material. CZM avoids unrealistic stress singularity at the crack tip by assuming that the stress at the FCT is always equal to the material ultimate strength.

In a two-parameter CZM, the ultimate strength along with the area under the stress-strain curve, or fracture toughness, completely defines the model. In a three-parameter model, the slip corresponding to the ultimate strength is considered as the third parameter.

The Cohesive Zone Models for simulating the FRP-concrete interface in the literature can generally be classified as linear, bilinear, or nonlinear models. The studies on the effect of the shape of the CZM show that the detailed shape of the cohesive law are less important than the fracture toughness and the interface strength (Qiao and Chen, 2008).

Högberg (2006) showed that regardless of shape, CZM's for all of the different modes have the same equations and the same shape, but are different in magnitude. Figure 2-8 shows a typical, bilinear CZM. It can be seen that the shape of the bilinear traction-separation law for all 
of the three pure modes has the same shape. It should be noted that in Mode I loading, fracture does not occur if the direction of the applied load is changed, i.e., there is no fracture under the compression; this is why in Figure 2-8 (b), the curve is only drawn in the positive side.

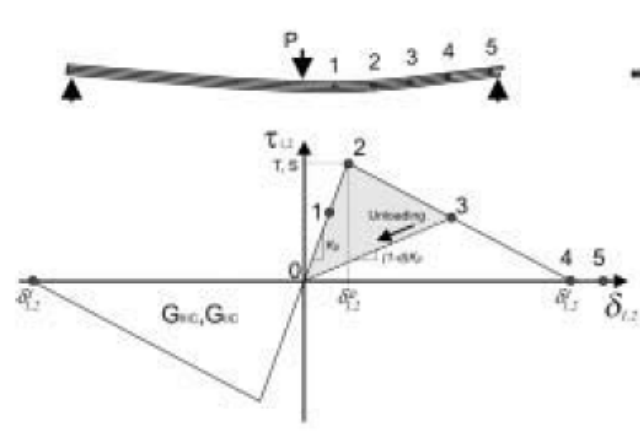

a) Mode II or Mode III

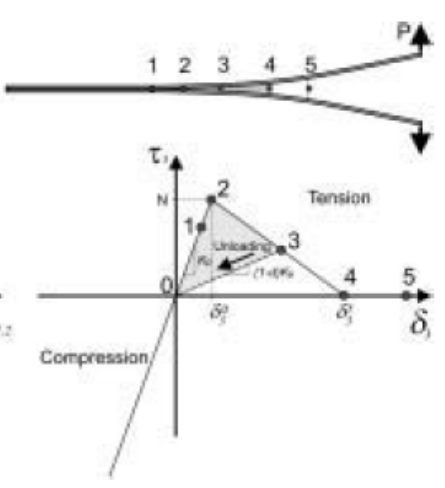

b) Mode I

Figure 2-8 Typical Bilinear Traction-Separation Law (adopted from Camanho PP and Dávila, 2002)

The area under traction-separation curve gives the fracture toughness which as mentioned earlier is a material property:

$$
G_{i C}=\int_{0}^{\delta_{j}^{f}} \tau_{j} d \delta_{j} \quad i=I, I I, I I I \text { and } j=1,2,3
$$

where $\tau_{\mathrm{j}}$ is the traction in a single mode loading and $\delta_{j}^{f}$ is the corresponding relative displacement upon failure. In using a bilinear law, the fracture toughness will be equal to the triangle area shown in Figure 2-8.

$$
G_{i C}=\int_{0}^{\delta_{j}^{f}} \tau_{j} d \delta_{j}=\frac{S_{j} \times \delta_{j}^{f}}{2}
$$

where $S_{j}$ is the strength of corresponding pure mode, which is again another material property.

It is worth mentioning that the fracture mechanics models alone cannot capture the difference between a cohesive failure and an adhesive one, when delamination occurs between concrete and FRP. This is simply due to the fact that CZM's consider the cohesive zones from a global point of view. In order to capture the stress intensities and the transition between cohesive and adhesive failure modes, other tools such as finite element simulations should be used.

The CZM's can be categorized in two groups: uncoupled and coupled. In uncoupled models, it is assumed that stress-deformation relations in Mode I and Mode II are completely independent 
from each other. The energy release rates in Mode I, $G_{I}$, and that of Mode II, $G_{I I}$, are defined as the areas under the corresponding CZM curves. The total energy release rate is then defined as the sum of $G_{I}$ and $G_{I I}$. This approach was shown to have the capability of capturing essential properties of adhesive joints.

On the other hand, in coupled models, it is assumed that stress-deformation relations in the two modes are coupled. Coupled CZM's themselves can be divided into two categories: those that allow for different fracture energies in different mode mixities and those which do not. A drawback of the uncoupled models is that the fracture energy is assumed to be the same for all mode mixities, while it is well known that the Mode II fracture energy is much greater than that of Mode I.

Among the coupled models, those developed by $\mathrm{Xu}$ and Needleman (1993) and that of Högberg (2006) can be mentioned. The drawback of Xu and Needleman's model is that the shape of their law is restricted to an exponential form. Plus, it was shown by van den Bosch et al. (2006) that this model does not simulate the interface behavior correctly unless the fracture energies of Mode I and Mode II are equal, which is not always the case.

\subsection{Previous Research}

As FRP technology has been utilized more frequently to rehabilitate and retrofit concrete bridges over the past two decades, researchers all over the world have worked to develop constitutive laws to model FRP-concrete interface behavior, and from there, predict the ultimate delamination of the FRP.

All of these proposed models can be categorized as follows: (a) conventional approach (strength-based models), (b) linear elastic fracture mechanics (LEFM) approach, (c) Cohesive Zone Model approach (based on nonlinear elastic fracture mechanics), and (d) damage mechanics.

The conventional empirical models have been obtained by data regression from different test setups with a variety of specimen geometries and material properties. Therefore, these models can only be applied to similar cases. In these models, it is assumed that debonding occurs when the calculated interfacial stress reaches the interface strength (Wang 2007). In order to avoid the singularity problem some researchers (Taljsten, 1996; Bazant et al., 1996) used LEFM to obtain the Mode II fracture energy of the CFRP-concrete and steel-concrete interfaces. However, the 
basic assumption of linear elastic fracture mechanics (LEFM) is that the size of deformation zone is small, compared to the crack size. This is obviously not the case for concrete, or even some composite materials.

The fracture mechanics-based models are generally more appealing to researchers than strength-based models, since the same single model can predict both crack initiation and propagation. On the other hand, since most of these models are obtained by means of simple test setups like single or double shear tests, (see section 1.3) the complicated stress states in a real structure and along with the interactions among different cracks in different orientations have been neglected.

The Cohesive Zone Model (CZM) was introduced by Barenblatt (1962) and Dugdale as an alternative to overcome LEFM's limitations with respect to large deformation zones in the crack vicinity, and is gaining more popularity in modeling the FRP-concrete interface behavior. The first CZM was developed by Barenblatt (1962) in order to simulate the Mode I fracture. In this model, the relationship between traction and separation, which are normal to the fracture surfaces, were considered. Here, the unphysical stress singularity at the crack tip in the traditional linear elastic fracture mechanics is removed. Later on, Cohesive Zone Models were extended to Mode II failure, where instead of normal traction and separation, tangential ones are considered (Högberg, 2006). Although the CZM are more appealing comparing to the other models, none of the existing models are accepted generally.

Many researchers have used the nonlinear fracture mechanics approach in their investigation of FRP-concrete interface (Yuan et al., 2001; Wu et al., 2002; Yuan et al., 2004; Wang, 2006, 2007a, b; Mazzotti et al, 2004; Ali-Ahmad et al., 2005; Dai et al. 2005). The results of experimental research conducted by Chajes et al., 1996; Bizindavyi and Neale, 1999; Yao et al., 2005 well supports the application of this nonlinear fracture mechanics approach. The use of the damage mechanics approach to model concrete structures strengthened by externally bonding FRP laminates sounds promising, but is not popular among researchers yet.

Consequently, despite these efforts to develop a constitutive law to model FRP-concrete interface behavior, there is still more room for research in this area, and a general well-accepted law which is not affected by the geometry or material properties of the specimen or the test configuration is still needed to be developed (Karbhari, 2003). 
Experimental research has shown that three major failure modes can be found in the FRP strengthened structures: (I) debonding of FRP from concrete or delamination, (II) adhesive failure which occurs in the epoxy layer, (III) FRP rupture. Since FRP strength is much higher than that of concrete and adhesive, the latter form of failure, i.e., FRP rupture rarely occurs. The most common mode of failure is debonding of FRP from concrete substrate, or delamination. Thus, obtaining comprehensive knowledge of the state of the FRP-concrete interface, both stresswise and energy-wise is imperative for design and maintenance purposes.

In a more detailed classification, Karbhari and Zhao (1998) defined five different failure modes between FRP and concrete in an externally reinforced beam, based on the location of the failure: (a) interfacial failure between concrete and adhesive, (b) cohesive failure in the adhesive, (c interfacial failure between the adhesive and the composite, (d) alternating crack path between the two interfaces, (e) peel failure into concrete (See Figure 2-9).
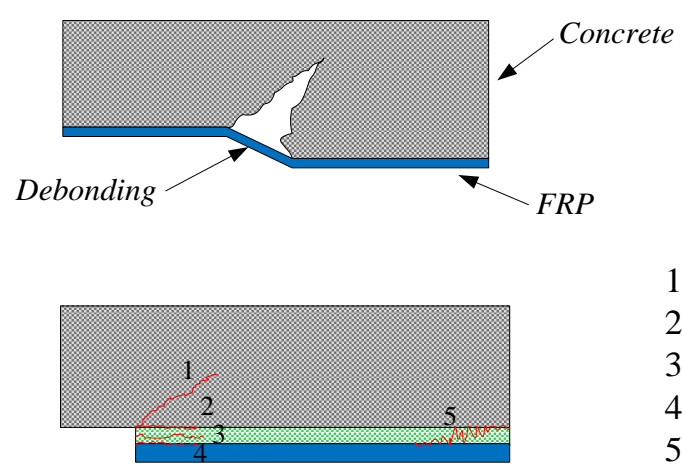

1. Cohesive Failure

2. Interfacial Failure between Concrete and Adhesive

3. Adhesive Failure

4. Interfacial Failure between FRP and Adhesive

5. Alternating Crack path between the two Interfaces

Figure 2-9 Interfacial Failure Modes (adopted from Karbhari et al., 1997 )

In Reinforced Concrete (RC) beams flexurally-strengthened with FRP laminate bonded to the tension side, delamination may occur at a major flexural crack or flexural-shear crack. The stress state of concrete-FRP interface during this type of delamination can be modeled as the stress state of a single shear specimen in the interface (Yuan 2004).

Among different definitions found for the "interface" in literature, one of the best definitions is provided by Yuan et al (2004) as the adhesive layer which represents not only the deformation of the actual adhesive layer but also that of the materials adjacent to the adhesive layer. Throughout this study, this definition of interface is considered.

Several constitutive laws are suggested by different researchers to model the state of FRPconcrete interface subjected to pure shear loads considering different parameters (Chajes et al., 
1995, 1996; Bizindavyi and Neale, 1999; Taljsten, 1997; Maeda et al., 1997; Yuan et al., 2001 and 2004; Yao et al., 2004; Wang, 2006 and 2007a; Yoshizawa et al., 2000; Chen and Teng 2001; Nakaba et al. 2001; Mazzotti et al, 2004; Wu et al., 2002; Ali-Ahmad et al., 2005; De Lorenzis et al. 2001 but none of them are generally accepted. Therefore, considering the importance of such a common and dangerous failure mode, developing a general well-accepted constitutive law to model the FRP-concrete behavior is of a great importance. Some of these models are briefly explained in this chapter.

Taljsten (1996) was one of the pioneers of applying LEFM to study the FRP or steel-bonded concrete beams. He showed that the maximum transferrable load in an adhesive joint is directly proportional to the square root of the interfacial fracture energy:

$$
P_{\max } \propto \sqrt{G_{f}}
$$

Considering a symmetric and a non-symmetric setup as shown in Figure 2-10, he found a rough estimate for the maximum transferrable load in each setup as follows:

(a)

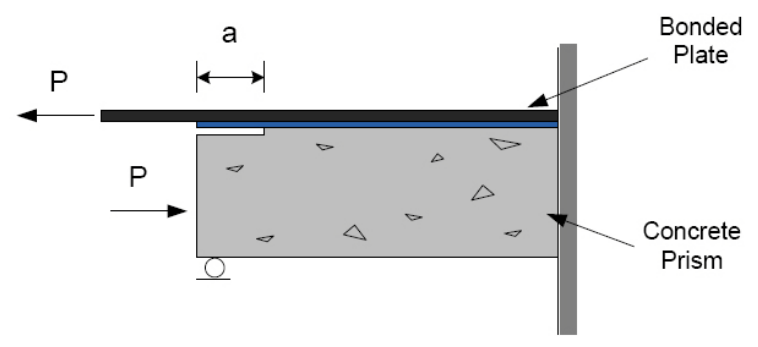

(b)

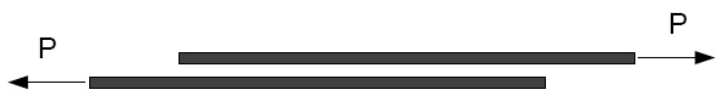

Figure 2-10 (a) non-symmetric set up (b) symmetric set up (adopted from Taljsten; 1996)

$$
\begin{array}{ll}
P_{\max }=2 b \sqrt{E t G_{f}} & \text { Symmetric Joint } \\
P_{\max }=b \sqrt{\frac{2 E_{1} t_{1} G_{f}}{1+\alpha}} & \text { Non-Symmetric Joint }
\end{array}
$$

where $\alpha=\frac{E_{1} t_{1}}{E_{2} t_{2}}, E_{1}$ and $t_{1}$ are modulus of elasticity and thickness of concrete respectively and $E_{2}$ and $t_{2}$ are those of FRP, $G_{f}$ is the interfacial fracture energy, and $b$ is the width of the strengthening plate. 
Taljsten (1997) studied the behavior of steel-concrete and CFRP-concrete interfaces and in doing so, introduced the concept of the effective bond length, or anchor length, which is the minimum length of the steel or FRP plate that a plate longer than that will not carry any additional load (See Figure 2-11). The existence of the effective bond length was also shown by several other researchers afterwards (Chajes et al.; Maeda et al.; de Lorenzis et al; Yuan et al. 2001, 2004; Nakaba et al., 2001; M. Ali-Ahmad et al., 2004; Kamel et al., 2006, Bizindavy and Neale). Later, the effective bond length was defined by Yuan et al. (2004) as the bond length required for reaching $97 \%$ of the applied load. It was also shown that the debonding process is related to the strain field in the FRP, and that there is a critical strain level on the bonded plate, where concrete starts to fracture.

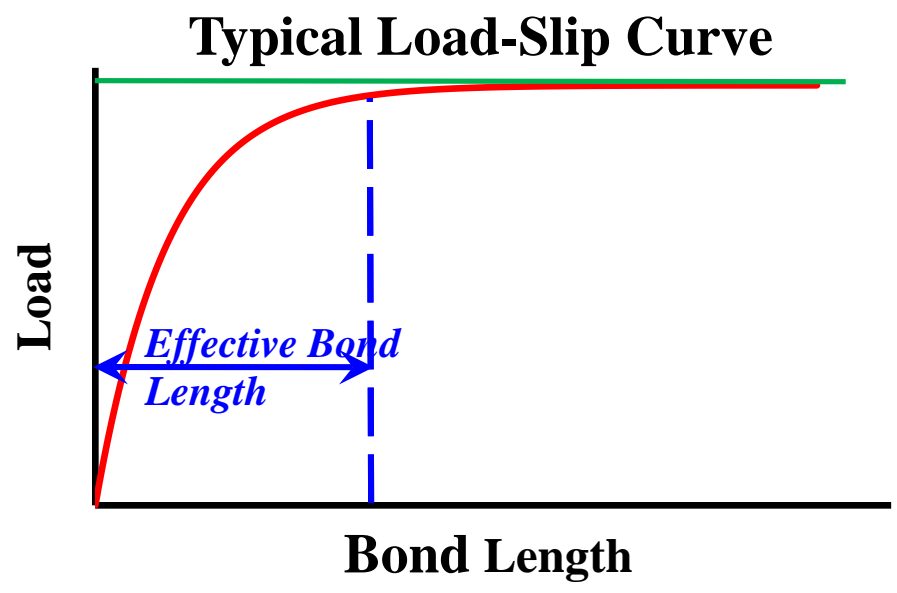


Figure 2-11 Effective Bond Lenggh

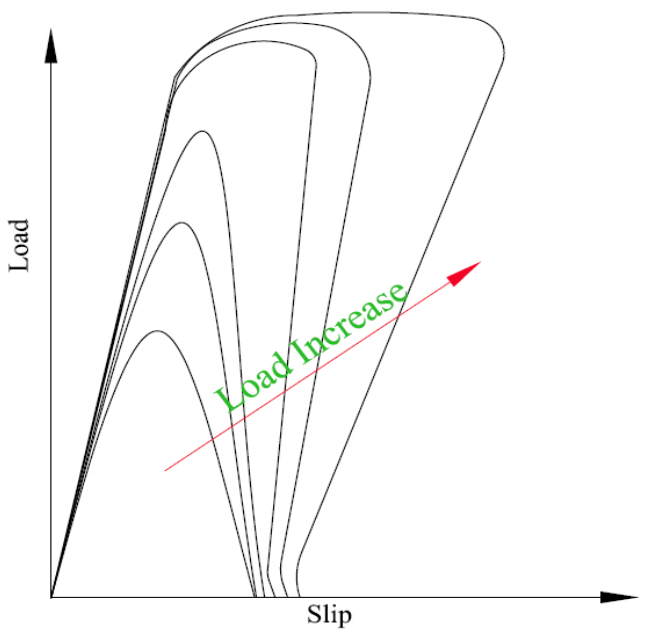

Figure 2-12 Effective Bond Length (adopted from Ali-Ahmad et al., 2007)

Ali-Ahmad et al. (2007) in their numerical analysis showed that increasing the bond length beyond the effective length results in snapback, which is a "portion of the load response where both the load and displacement decrease simultaneously.”

The effect of the specimen size on the strength of quasi-brittle materials was shown by Bazant et al. (1996). That is why the test results reported by different researchers who have used different specimens with different geometries and material properties are not comparable.

Chajes et al. (1996) used a single shear test setup to study the bond and load transfer mechanism in FRP plates bonded to concrete. Results showed that the concrete surface preparation affects interfacial strength. It was also suggested that the ultimate interfacial shear strength is proportional to the square root of compressive strength of concrete, $\sqrt{f_{c}^{\prime}}$.

Bizindavy and Neale (1999) studied the shear mode between the FRP sheets and concrete beams through a set of single shear test experiments. Traditional data analysis was performed by analyzing the strain distribution on the FRP recorded by strain gages. It was shown that the effective bond length is a function of the properties and geometry of the specimens, which also depends on surface preparation. It was further shown that the bond strength of externally bonded FRP laminates mainly depends on the quality of the surface preparation, and the quality of the concrete itself.

de Lorenzis et al. (2001) investigated the parameters that could probably affect the behavior of bond between FRP and concrete concluded that the FRP width does not have any effect on the 
bond strength. Subramaniam et al. (2007) studied the effect of the width of the FRP laminate on the interface properties in more details. They concluded that the fracture properties of the central portion of the interface away from the edges are independent of the width of the FRP which is in accord with de Lorenzis et al.'s (2001) conclusion. The difference between the two however, is that the maximum load is higher for wider FRP laminates. Mazzotti et al. (2008) also reported that decreasing the plate width increases the maximum shear stress, while no width effect was observed on fracture energy and the delamination load. Kamel et al. (2006), however, reported that the interface stiffness is more dependent on the length-to-width ratio of the FRP, rather than the length of the FRP. This discrepancy might be related to the nature of their test, which was a modified pull-apart test as shown in Figure 2-13.

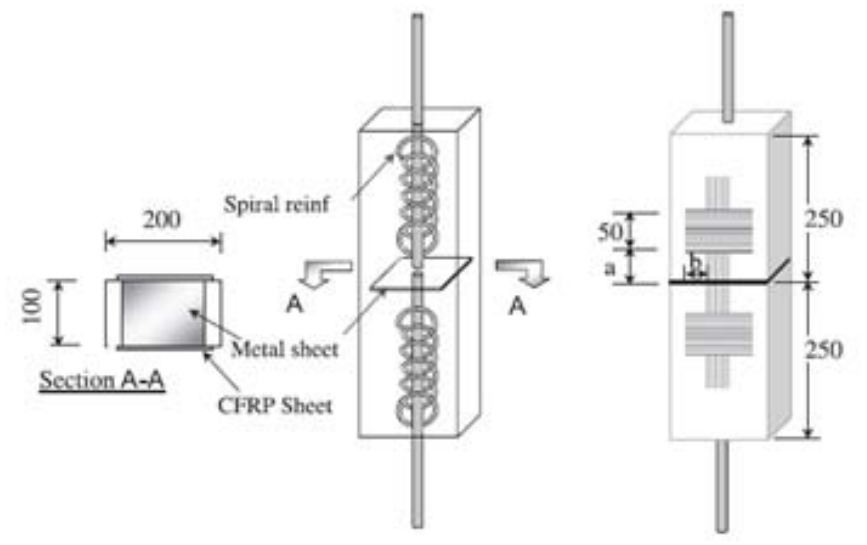

Figure 2-13 Pull-apart test (Adopted from Kamel et al. (2006))

Dai et al. (2005) studied the behavior of FRP-concrete interface under shear loads for different FRP materials (carbon fibers, aramid fibers, and glass fibers), FRP stiffness, and different adhesives. In their proposed model, the strain distribution was obtained based on the relative slip between FRP and concrete, recorded by means of two LVDT's at both ends of the FRP. In other words, the strain was not recorded to obtain their model. Their model was developed based on the assumption that there is unique relationship between the FRP strain and the interfacial slip as follows:

$\varepsilon=f(s)$

where $\varepsilon$ is the FRP strain and $\mathrm{s}$ is the relative slip between the FRP and concrete. An exponential curve was fitted to the experimental results of shear tests as follows:

$\varepsilon=f(s)=A(1-\exp (-B s))$ 
where $A$ and $B$ are experimental constants. Using the chain rules of derivatives, the strain gradient is obtained as follows:

$$
\frac{d \varepsilon}{d x}=\frac{d f(s)}{d s} \frac{d s}{d x}=\frac{d f(s)}{d s} \varepsilon=\frac{d f(s)}{d s} f(s)
$$

The following equation stands between the interfacial shear stress and the gradient of the strain on the FRP:

$$
\tau=E_{f} t_{f} \frac{d \varepsilon}{d x}
$$

Finally, substituting Equations (2-16) and (2-17) into Equation (2-18), the interfacial shear stress is obtained as a function of relative slip as follows:

$$
\tau=A^{2} B E_{f} t_{f} \exp (-B s)(1-\exp (-B s))
$$

where $E_{f}$ and $t_{f}$ are the FRP modulus of elasticity and thickness. $G_{f}$ is the interfacial fracture energy obtained using the following equation:

$$
F_{\text {max }}=b_{P} \sqrt{2 E_{P} t_{P} G_{f}}
$$

The empirical constants of this model are obtained by means of regression techniques, given the mechanical properties of both adhesive and FRP. The problem is that by increasing the FRP, stiffness or adhesive shear modulus $B$ increases infinitely. They observed that the effects of adhesives and concrete are more pronounced in the interfacial behavior comparing to the effect of FRP stiffness.

Ali-Ahmad et al. (2006) developed another model for FRP-concrete interface based on single shear tests by traditional approach. However, the strain distribution was determined using an optical technique called Digital Image Correlation (DIC). In order to obtain the strain at the centerline of the FRP, the strain over a width of $10 \mathrm{~mm}$ was averaged out to reduce the effect of material variation. One may ask why not the average of the strain across the whole width of the concrete was not utilized instead; the reason, as shown by Kamel et al. (2006), is that the strain at the edge of the CFRP is consistently higher than that of the centerline. Therefore, averaging the strains in the aforementioned width sounds reasonable. Although using the DIC technique is beneficial in providing a spatial field of strain, and avoids the errors introduced by the operator in the traditional method of using strain gages, it is too expensive and requires special equipment. This model was also developed by means of regression techniques. To begin with, an exponential curve in the following form was fitted to the strain data. 


$$
\varepsilon(y)=\frac{\alpha}{1+\exp \left(-\frac{y-y_{0}}{\beta}\right)}
$$

Where $\alpha, \beta, y_{0}$ are the empirical constants obtained by regression analysis of the measured strain by DIC. By substituting the above Equation into Equation (2-18), the stress-slip relationship was obtained.

Pan and Leung (2007 a) studied the effects of concrete compressive strength, concrete splitting tensile strength, concrete surface tensile strength, and also aggregate content of concrete on the FRP-concrete interface strength. Experiments were performed on single shear specimens. It was observed that the maximum transferrable load was not significantly affected by compressive strength, and splitting tensile strength of the concrete. However, concrete surface tensile strength and coarse aggregate content had a major effect on bond capacity. Consequently, they proposed an equation which related the interfacial fracture energy to the surface tensile strength and coarse aggregate content:

$$
G_{F}=f_{s t s}^{0.0173}(6.203 a+0.425) \quad \mathrm{N} / \mathrm{mm}
$$

Where $f_{s t s}$ is the concrete surface tensile strength, $a$ is the aggregate content and $G_{f}$ is the interfacial fracture energy obtained from Equation (2-18). The main reason for interfacial fracture energy independency from concrete compressive strength and splitting tensile strength is that delamination of the FRP from the concrete substrate is governed by the mechanical properties of the substrate closer to the interface, which for concrete is entirely different from the bulk properties. Hence, as compressive strength and splitting tensile strength are bulk properties there cannot be a reasonable correlation between these two properties and interfacial fracture energy.

As a second reason, the nature of concrete failure, which involves the formation, propagation and coalescence of microcracks, must be considered. Obviously, in compression testing, the stress distribution is uniform, whereas in splitting tension test, most of the failure plane is under similar stress. These tests are different from the failure process in the direct shear test, in which the load is applied only to the specimen surface.

Mazzotti et al. (2008) adopted Popovics’ nonlinear equation to analyze the data from single shear test experiments. Popovics’ equation (1973) which was originally introduced to relate 
concrete strength and the strain distribution is modified for the FRP-concrete interface as follows:

$$
\frac{\tau}{\tau_{\max }}=\frac{s}{s_{\max }} \cdot \frac{n}{(n-1)+\left(s / s_{\max }\right)^{n}}
$$

where $\tau_{\max }$ is the peak shear stress, $s_{\max }$ is the corresponding slip, and $n>2$ is an empirical constant governing the softening branch of the $\tau$-s curve. Starting from the strain data, shear stress is obtained using Equation (2-18), and the slip is obtained by integrating the strain over the bond length as follows:

$$
s(x)=\int_{x_{0}}^{x} \varepsilon(x) d x=s\left(x_{i+1}\right)+\frac{1}{2} \frac{\varepsilon_{i+1}-\varepsilon_{i}}{x_{i+1}-x_{i}}\left(x-x_{i}\right)^{2}+\varepsilon_{i}\left(x-x_{i}\right) ; \quad x_{i} \leq x \leq x_{i+1}
$$

The three unknown parameters of Equation (2-23), i.e., $\tau_{\max }, s_{\max }$, and $n$ are obtained via least square minimization. The fracture energy is calculated from Equation (2-20) and is used for the minimization process. Different values of $\tau_{\max }, s_{\max }$, and $n$ were obtained for different bond lengths and different test setups.

Mazzotti et al. (2008) also investigated the effect of the existence of a notch between FRP and concrete at the loading end. They observed that in case of the existence of a primary notch at the beginning of the bond, the results are more representative of the material behavior. According to their experiment, when there is no primary notch, the failure will occur along with the separation of a small triangular concrete portion in the traction side of the specimen (wedge failure).

The maximum shear stress obtained using the data of the test set up without the primary notch was reported to be about half of what obtained using the specimens with the primary notch although the corresponding slip in both cases is almost the same. Obviously, the fracture toughness of the former will be half of that of the latter. Lu et al. (2005), on the other hand, believed that as long as the bond length is not too short, the difference between the two specimens and the triangular portion of the concrete does not have a significant effect on the interface law. Ferracuti (2006) showed that when there is no primary notch, the ultimate load may reduce up to $60 \%$ compared to that of a specimen with a primary notch; although with increasing the bond length this effect will be reduced and when the bond length tends to infinity, both forces would be the same. However, most researchers have used the specimens with the primary notch. 
In most models developed for FRP-concrete interface, the basic assumption is that the bond length tends to infinity; thus these equations can only be used for the experiments in which the bond length is sufficiently long, i.e., longer than the effective bond length.

Same as Mazzotti et al. (2008), Nakaba et al. (2001) also used Popovics’ equation (Equation (2-23)) for developing their model. However, they used both a different approach and experimental setup.

The specimen chosen for their research was double shear test set up. In contrast to Mazzotti et al. (2008), who had obtained the maximum shear stress, $\tau_{\max }$, through least square minimization, Nakaba et al. (2001) proposed an exponential function of concrete compressive strength as follows:

$\tau_{\max }=3.5 f_{c}^{0.19}$

A schematic view of their model can be seen in Figure 2-14.

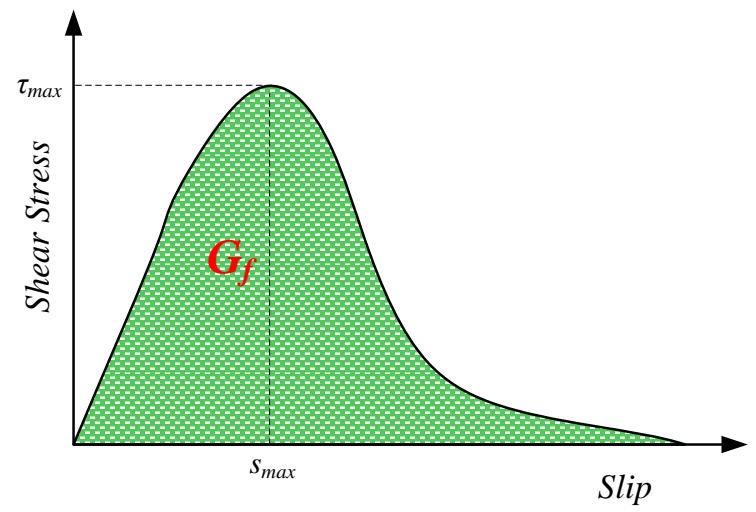

Figure 2-14 Schematic View of Nakaba et al.'s Model

By choosing types of fiber and concrete substrate as the test variables, it was discovered that the maximum interfacial shear stress, $\tau_{\max }$, is not influenced by the type of FRP, but increases with the concrete compressive strength. Given the material properties used in this experimental work, this model can be adapted over the range of concrete compressive strengths of 24-58 MPa.

Pham and Al-Mahaidi (2007) also adopted Popovics’ equation, Equation (2-23), to simulate the CFRP-concrete interface behavior under single shear tests. Based on Nakaba et al.’s prior work, (2001), the value of " $n$ " was chosen equal to 3, in order to simplify the problem, and find the two unknowns, $\tau_{\max }$ and $s_{\max }$.

Although in Nakaba et al.'s model, the interface shear stress is dependent on the concrete characteristics, Coronado and Lopez (2006) changed the tensile strength of concrete between 
$0.5 f_{t}$ and $2 f_{t}$ (where $f_{t}$ is the tensile strength of concrete) and found out that debonding is insensitive to concrete strength. However, the fracture energy of the interface was introduced as a necessary tool to predict the plate debonding accurately. It was also reported that there is a threshold for the FRP area, below which, the FRP ruptures. Interestingly, in the numerical part of their study, they found out that modeling the concrete using the coarser mesh yields the results that are much closer to the experimental results.

By the same token, Qiao and Chen (2008) also concluded that the concrete tensile strength does not have any effect on the failure of the FRP-concrete interface. The reason is that the interface cohesive strength is higher than the concrete tensile strength; The interface failure is basically the cohesive failure of concrete along the interface. Thus, it has no effect on the interface fracture behavior and the interfacial load-displacement curve does not change by varying the concrete tensile strength.

Yao et al. (2005) also used single shear test experiments to show that the interface strength is dependent on specimen geometry.

In their thorough finite element investigation of interfacial stresses in a reinforced concrete beam strengthened by an externally bonded soffit plate, Teng et al. (2002) clearly illustrated that numerical models can overcome the simplifying assumptions implicit in analytical methods. The simplifying assumption made in all the analytical models is that the stress does not vary across the adhesive thickness. This is not the case in real life.

Wang and Davalos (2004) proposed a linear-exponential traction-separation law for plate debonding failure of RC beams strengthened with either steel or FRP plates. The proposed model was implemented in ABAQUS as a three-dimensional interface element later on. The proposed traction separation law can be expressed as follows:

$$
t_{i}= \begin{cases}E_{i} \hat{\delta}_{i} & \text { if } \hat{\delta}_{i} \leq \delta_{c i} \\ E_{i} \hat{\delta}_{i} e^{1-\hat{\delta}_{i} / \delta_{c i}} & \text { if } \hat{\delta}_{i}>\delta_{c i}\end{cases}
$$




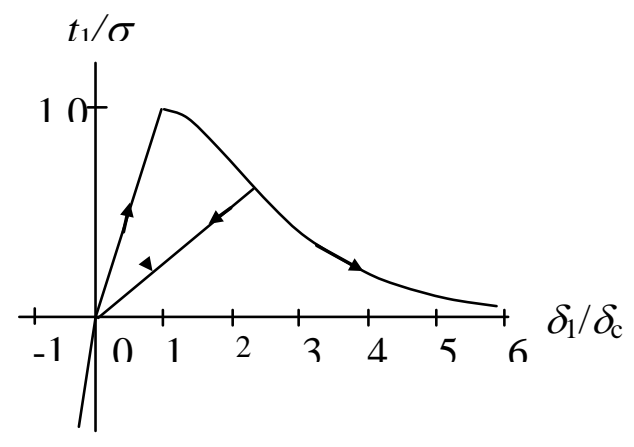

(a)

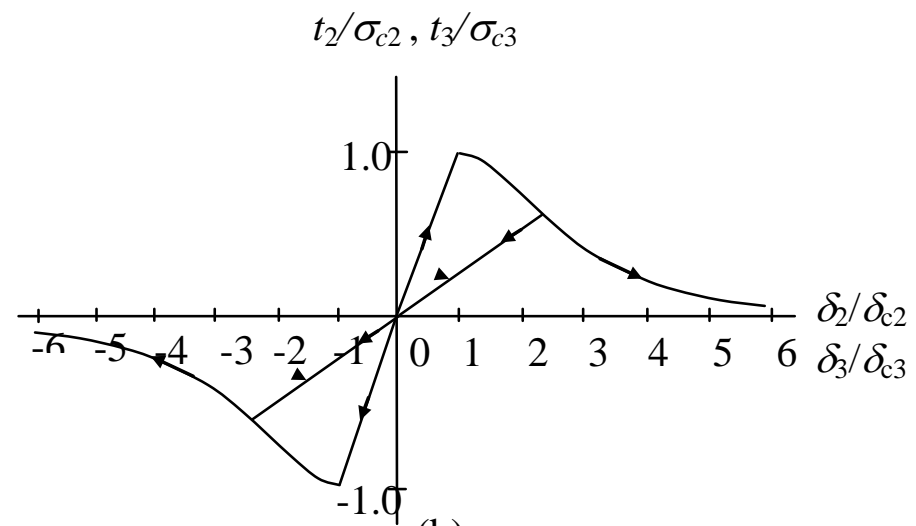

(b)

Figure 2-15 Linear-Exponential Traction-Separation Law; (a) Mode I, (b) Modes II and III (Adopted from Wang and Davalos (2004))

In order to prevent penetration under compression in Mode I, a relatively high stiffness was assumed when $\delta_{1}<0$ (See Figure 2-15). For pure Modes II and III, the traction-separation law has the same shape as Mode I loading, whereas for $\delta_{2}, \delta_{3}<0$ it is anti-symmetric with respect to its origin.

Camanho and Dávila (2002) developed a bilinear interface constitutive law for composite laminates under mixed mode loading as shown in Figure 2-16. Although this model was not developed for FRP-concrete interface, as the concept is the same and researchers like Qiao and Chen (2008) have adopted this method for FRP-concrete interface later on, this model is briefly explained here.

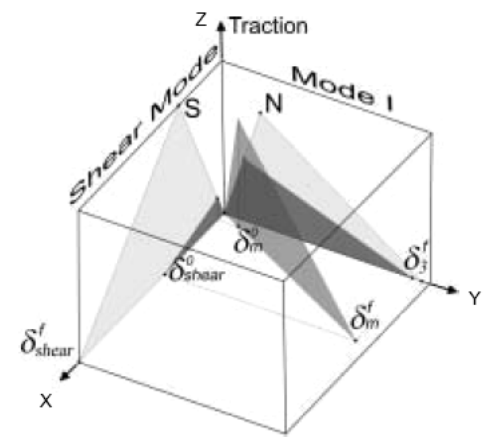

Figure 2-16 Bilinear Mixed Mode Cohesive Law (adopted from Camanho and Dávila (2002))

In this model, which was defined using CZM and a damage mechanics approach, the onset of delamination was predicted using a strength-based criterion through a quadratic interaction between traction stresses, while the delamination propagation was predicted using mixed mode 
fracture energy criteria i.e., power law and Benzeggagh-Kenane criteria. The quadratic criterion for predicting the damage initiation is as follows:

$$
\left(\frac{\left\langle\tau_{1}\right\rangle}{\tau_{n}}\right)^{2}+\left(\frac{\tau_{2}}{\tau_{s}}\right)^{2}+\left(\frac{\tau_{3}}{\tau_{t}}\right)^{2}=1
$$

where $\tau_{n}$ is the interlaminar tensile strength, $\tau_{s}, \tau_{t}$ are the interlaminar shear strengths, and $\langle x\rangle=\left\{\begin{array}{l}0 \Leftarrow x \leq 0 \\ x \Leftarrow x>0\end{array}\right.$ as the normal compressive stress does not cause any damages (Mode I).

Power Law criterion is generally expressed as follows:

$$
\left(\frac{G_{I}}{G_{I C}}\right)^{\alpha}+\left(\frac{G_{I I}}{G_{I I C}}\right)^{\alpha}=1
$$

$\mathrm{B}-\mathrm{K}$ criterion is defined as follows:

$$
G_{I C}+\left(G_{I I C}-G_{I C}\right)\left(\frac{G_{\text {shear }}}{G_{T}}\right)^{\eta}=G_{C} \text {, with } G_{T}=G_{I}+G_{\text {shear }}
$$

This constitutive model was used to define interface elements to be implemented in ABAQUS. These elements are placed between layers of solid elements in order to model the interface. Delamination initiation and propagation are simulated without having any information about the location, size or growth direction.

Qiao and Chen (2008) developed a bilinear damage CZM to simulate the Mode I fracture of FRP-bonded concrete beams using the same approach as Camanho and Dávila (2002). The proposed model consisted of three constitutive laws for different components: (a) a bilinear damage cohesive law to simulate the adhesive-concrete debonding; (b) a concrete tensile plastic damage model adopted from literature to simulate the cohesive failure of concrete; (c) a general Hooke's law for orthotropic elastic materials for the FRP.

This model was implemented in ABAQUS to model the failure modes of 3-point bending beams. In this model, three failure modes were identified: (1) complete adhesive-concrete interface debonding, representing a weak bond; (2) complete concrete cohesive cracking near the bond line, representing a strong bond; and (3) a combined failure of interface debonding, and concrete cohesive cracking. Figure 2-17 shows the bilinear damage cohesive law as defined by Qiao and Chen (2008). 


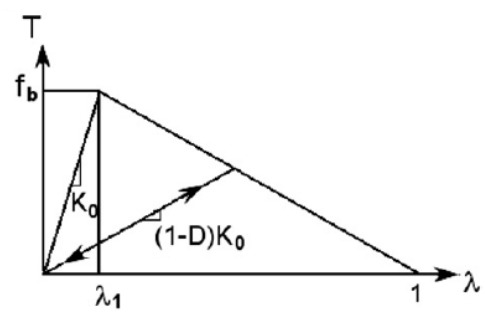

Figure 2-17 Bilinear damage cohesive law (adopted from Qiao and Chen (2008))

In this model, the same strength-based criterion as Camanho and Dávila (2002) (Equation (2-27)), was used to depict damage initiation, while a fracture mechanics-based approach, (the power law criterion) with $\alpha=1$ (Equation (2-28)), was used for damage evolution. The only difference with the aforementioned model is that $\tau_{3}$ was not considered in Qiao and Chen's model.

Following the interface constitutive laws which have been implemented in Finite Element Packages, the normalized CZM developed by Högberg (2006) must be discussed. As with Camanho and Dávila (2002), this model was implemented in ABAQUS to simulate double cantilever beam responses under mixed mode loadings. A damage formulation was used to capture differences between loading and unloading.
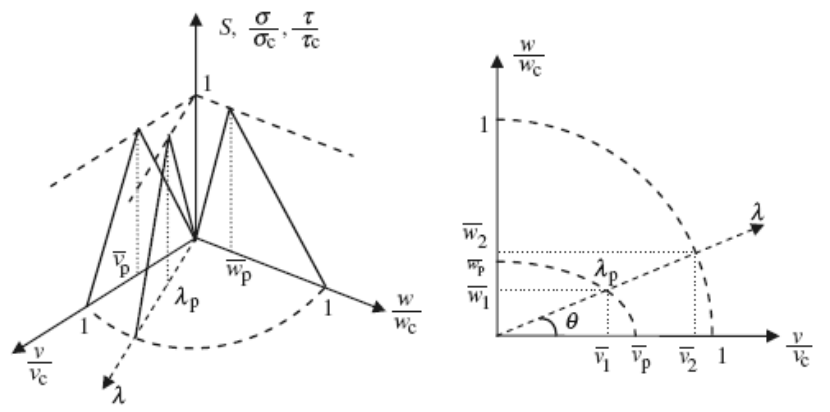

Figure 2-18 Graphical Representation of CZM developed by Högberg (2006) (adopted from Högberg (2006))

Among the analytical models developed to model the FRP-concrete interface behavior under Mode II loading, those developed by Yuan et al. (2004) and Wang (2006) can be mentioned.

The analytical model presented by Yuan et al. (2004) was based on a bilinear bond-slip model, and was claimed by the authors to predict the entire debonding process of FRP-concrete joints under Mode II loading (See Figure 2-19). Their model provided closed form solutions for interfacial shear stress as well as load-slip response at different load levels. Although bilinear 
models are easy to deal with on paper, due to the sudden change of slope, their implementation in finite element packages is difficult and requires special caution.

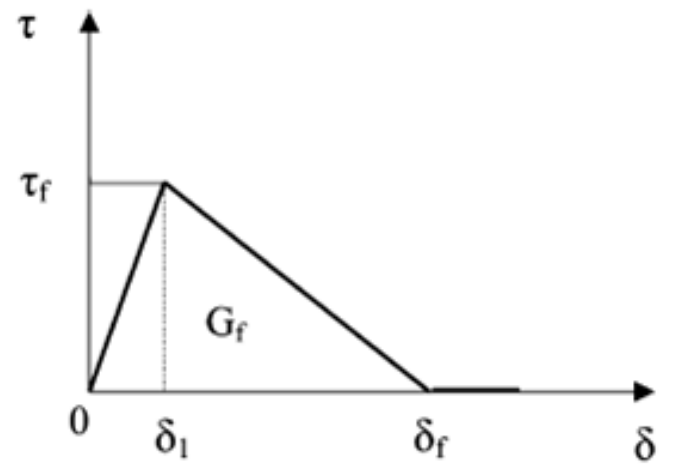

Figure 2-19 Bi-Linear Model (adopted from Yuan et al. (2004))

An analytical solution was suggested for the effective bond length considering a bilinear model as follows:

$$
l_{e}=a+\frac{1}{2 \lambda_{1}} \ln \frac{\lambda_{1}+\lambda_{2} \tan \left(\lambda_{2} a\right)}{\lambda_{1}-\lambda_{2} \tan \left(\lambda_{2} a\right)}
$$

where:

$$
\begin{aligned}
& \lambda_{1}=\sqrt{\frac{\tau_{f}}{\delta_{1}}\left(\frac{1}{E_{p} t_{p}}+\frac{b_{p}}{b_{c} E_{c} t_{c}}\right)} \\
& \lambda_{2}=\sqrt{\frac{\tau_{f}}{\delta_{f}-\delta_{1}}\left(\frac{1}{E_{p} t_{p}}+\frac{b_{p}}{b_{c} E_{c} t_{c}}\right)}
\end{aligned}
$$

where:

$b_{p}, t_{p}, E_{p}=$ width, thickness, and modulus of elasticity of FRP laminate respectively.

$\mathrm{b}_{\mathrm{c}}, \mathrm{t}_{\mathrm{c}}, \mathrm{E}_{\mathrm{c}}=$ width, thickness, and modulus of elasticity of concrete beam respectively.

$\tau_{\mathrm{f}}, \delta_{1} \quad=$ maximum shear stress and corresponding slip.

$\delta_{\mathrm{f}} \quad=$ slip when fracture occurs (shear stress goes to zero at this point).

In order to derive Equation (2-30), the effective bond length was defined as "the bond length required reaching to $97 \%$ of the applied load.”

Maeda et al. (1997) proposed another relationship for estimating the effective length as follows:

$$
l_{e}=\frac{25350}{\left(t_{p} E_{p}\right)^{0.58}}
$$


where $t_{p}$ and $E_{p}$ are the FRP thickness and modulus of elasticity, respectively.

Wang and Zhang (2008) further expanded this model to account for both Mode I, and Mode II debonding.

The other example of using bilinear models to simulate FRP-concrete interface was used by Wang (2006). In this model, the debonding process was divided into 5 stages: (1) Elastic-Elastic; (2) Elastic-Softening-Elastic; (3) Elastic-Softening-Softening- Elastic; (4) Elastic-SofteningDebonding-Softening-Elastic; and (5) Softening-Debonding-Softening-Elastic.

Taking both RC beam and FRP plates as linear elastic Euler-Bernoulli beams, closed form solutions were obtained for each stage. Experimental results of this experiment have clearly shown that the CFRP-concrete interface law as a matter of fact must be rather nonlinear. In order to modify the bilinear model to have a better agreement with experimental results, Wang (2007) developed a new method for obtaining the interface law using J-integral.

Since the bilinear model does not account for the difference between the crack processing (bridging) and particle bridging (interlocking) failure modes and uses a single slope line for the softening part, he suggested the use of two different lines with different slopes for the softening branch in order to obtain a closer agreement with experimental results. Consequently, based on this new model, the interface is divided into four different zones: (1) Elastic zone, in which stress increases linearly with the slip; (2) Softening (cohesive zone) zone, in which stress decreases linearly with the slip; (3) Bridging zone, in which stress drops to a residual strength and decreases linearly with the slip to zero; and (4) Fully debonded zone, in which stress is zero and the FRP plate is fully separated from the concrete beam.

Since the Cohesive Zone Model and Bridging Zone Model are combined in order to derive this new model, he named his model Cohesive-Bridging. Although his analytical work was brilliant, Wang's model is difficult to implement in practice. This model is not a smooth curve, and is composed of four broken lines, making it even more difficult than the bilinear curve to implement in finite element packages. 

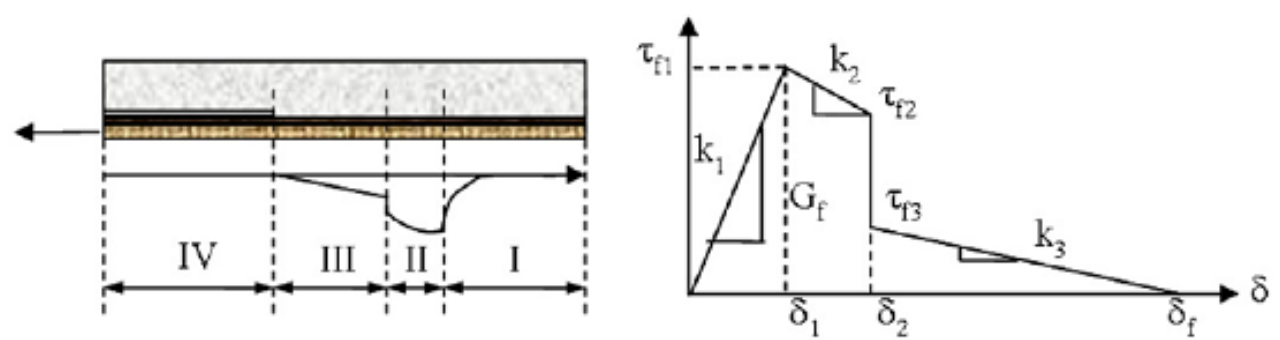

Figure 2-20 cohesive-bridging model (Adopted from Wang (2007 b))

Anil and Belgin (2008) summarized the twelve bond-strength models for CFRP-concrete bond and applied them to the experimental results from a series of four-point bending tests on notched CFRP bonded, single span concrete beams. In comparing the values of concrete compressive strength, bond length, and CFRP width, it was concluded that models containing more variables affecting the bond strength, such as bond length, FRP width and tensile strength of the substrate have a better agreement with experimental results. Finally they concluded that the model presented by Chen and Teng (2001) best fits the experimental data.

\subsection{Durability}

As the durability of FRP-interface is also studied in this research, it is worth reviewing some of the previous works on this issue.

The word 'durability' in this particular context, describes the ability of a structural system to maintain its initial performance-properties over time (Cousins et al., 1998).

All the repeated loadings have some effects on the durability characteristics of a structure. Alternating environmental conditions (e.g. wet-dry and freeze-thaw cycling effects, general temperature and climate changes), are among the most important environmental effects. Traffic loads and exposure to deteriorating chemicals are also sources of durability concerns as well; however, they are not the focus of this research.

Moisture is one of the most important factors in durability studies, as fluid transport is involved in most of the aforementioned environmental conditions. In the case of reviewing and analyzing the CFRP-concrete interface studies, more focus is devoted to the concrete substrate, as the composite counterpart is assumed to be impermeable to the moisture (Boyajian 2002).

Karbhari et al. (2003) defined the durability of a material or structure as "its ability to resist cracking, oxidation, chemical degradation, delamination, wear, and/or the effects of foreign 
object damage for a specified period of time, under the appropriate load conditions, under specified environmental conditions.”

Various long-term prediction models considering FRP and concrete durability issues are proposed by different researchers. Prediction models based on the Arrhenius equation, namely, Litherland et al. (1981), Phani and Bose (1987) Chen et al. (2006), are more popular in the literature, although none of these models are accepted universally. Moreover, whether the elevated temperature will alter the degradation mechanism which violates the fundamental assumption of using Arrhenius equation, is still a question open for debate. On the other hand, there is no long standing experimental data available to validate the proposed prediction models, and this is one of the most important reasons that there is no consensus on a particular model.

Karbhari (2003) provided an interesting summary of the current status of FRP durability in civil engineering applications. In his classification of the existing gap in the durability database for FRP materials used in civil engineering applications, he found the biggest gap in almost all external applications of FRP's in beams, slabs and columns. This means that both the data is currently unavailable (or inaccessible) and this data is critical for use of FRP composites in civil infrastructure.

The Arrhenius model was initially developed for gases, and describes a relationship between the rate of a reaction and the absolute temperature. The Arrhenius equation takes the following form:

$k=A \exp \left(\frac{-E_{a}}{R T}\right)$

where:

$k \quad=$ the Arrhenius degradation rate.

$A=$ a constant of the test condition.

$E_{a} \quad=$ the activation energy of the chemical reaction.

$R \quad=$ the universal gas constant $\left(8.3145 \mathrm{JK}^{-1} \mathrm{~mol}^{-1}\right)$.

$T \quad=$ the absolute temperature $(K)$.

In order for the Arrhenius equation to be valid, it is assumed that the degradation is controlled by a single, dominant mechanism. Using this equation, the load or any other parameters such as strength or corresponding to a given level of deterioration can be obtained. 
Litherland et al. (1981) were pioneers in utilizing and implementing Arrhenius equation to investigating the durability of civil engineering materials. They developed a model based on the Arrhenius equation to predict the strength of Glass Fiber Reinforced Composites (GRC) exposed to hot water at different temperatures. Using their model, they predicted the expected strengths of GRC over a hundred.

Phani and Bose (1986) developed a model based on the Arrhenius equation, which implements acousto-ultrasonic technique to predict the flexural strength of composite laminates after a specific amount of exposure to temperature. They defined the relationship between flexural strength of composite laminate, $\sigma(t)$, and exposure time, $t$, as follows:

$$
\sigma(t)=\left(\sigma_{0}-\sigma_{\infty}\right) \exp [-t / \tau]+\sigma_{\infty}
$$

Where $\sigma_{0}$ and $\sigma_{\infty}$ are the composite strengths at times 0 and $\infty$, respectively and $\tau$ is a characteristic time, which is dependent on temperature. According to their study, tensile strength decreased with increase of exposure time. Chen (2007) modified this model to predict the strength retention of GFRP bars.

Studying the durability of GFRP rods exposed to de-ionized water at $23,40,60$ and $80^{\circ} \mathrm{C}$, Prian and Barkatt (1999) found that although Arrhenius extrapolation is valid for short-term tests, the degradation rate in some environments can increase with time after a certain time period. This is attributed to being as a result of degradation of fiber-matrix interface; the $\mathrm{pH}$ value of the environment around the fiber can be increased as a result of matix dissolution, and leaching enhances the interface degradation.

Nkurunziza et al. (2005) classified the effect of moisture on composite materials as follows: 


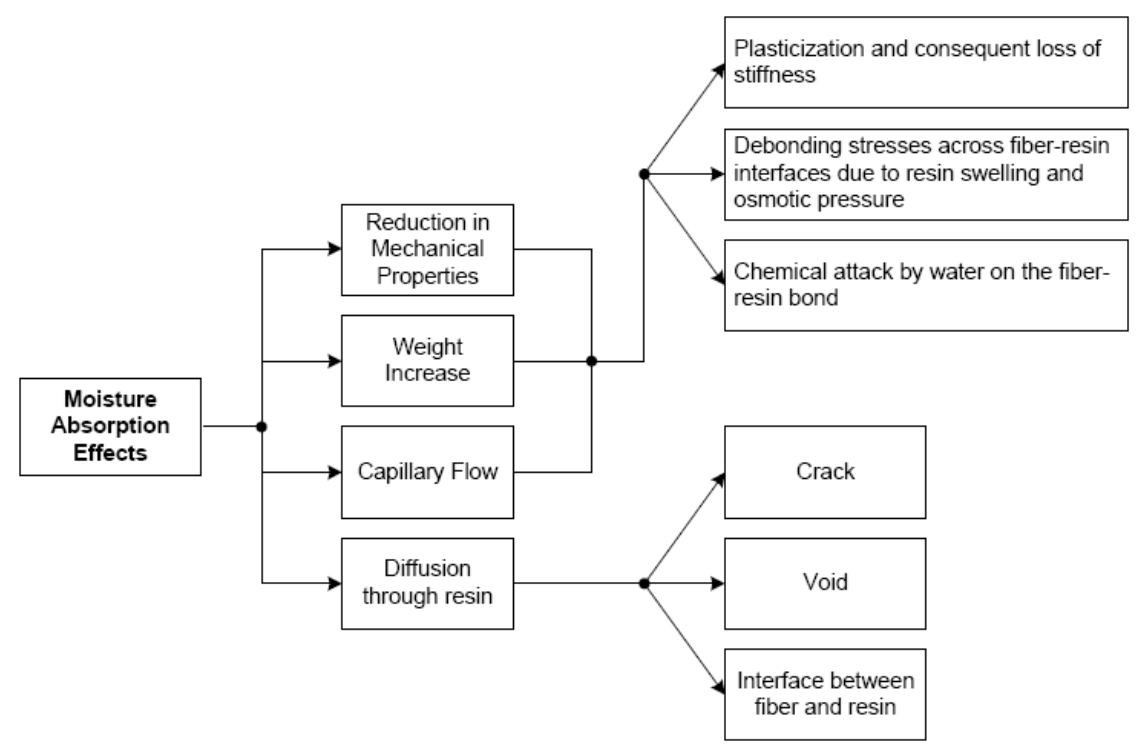

Figure 2-21 The overall effect of moisture on composite materials (Nkurunziza et al. ,2005)

\subsubsection{Experimental Case Study (Durability)}

Some of the prominent studies on the FRP-concrete durability are considered as follows in the order of the year they were published.

Karbhari et al. (1997) investigated the durability of FRP plate-concrete interface exposed to five different environmental conditions under mixed mode loading. Peel loads were applied at different angles relative to the bond line to determine the components of mode I and II interfacial fracture energies. In their research they used two different epoxies along with two different fibers, i.e. GFRP and CFRP. Their results revealed that CFRP-plated beams were less sensitive to environmental conditions compared to that of GFRP.

Karbhari and Zhao (1998) investigated the short term-durability of FRP strengthened concrete beams through a series of $4 \mathrm{pb}$ tests. Unidirectional laminates were externally bonded to $13 \times 2 \times 1$ in $^{3}$ concrete beams. Three different FRP systems, each having three plies, were used. Two were CFRP laminates and one was GFRP.

The effects of four different environmental conditions were investigated: (1) water condition; fresh water at ambient temperature, (2) sea water condition; synthetic sea water based on ASTM D1141 kept at ambient temperature; (3) frozen condition; the specimens were stored at $-15.5^{\circ} \mathrm{C}\left(4^{\circ} \mathrm{F}\right)$; and (4) freeze-thaw condition; cycled every 24 hours between room temperature $23^{\circ} \mathrm{C}\left(73^{\circ} \mathrm{F}\right)$ and $-15.5^{\circ} \mathrm{C}\left(4^{\circ} \mathrm{F}\right)$. The control specimens were stored at ambient temperature $23^{\circ} \mathrm{C}$ $\left(73^{\circ} \mathrm{F}\right)$. In case of exposure to water, the specimens were physically located in a place where 
water did not have direct contact with the FRP, in order to simulate the real conditions of a bridge site. Because in a real bridge, FRP is usually bonded to the bottom (tension side) of the beam, the only way moisture can get to interface is through the concrete from above. They observed that after 120 days of continuous moisture exposure (condition I), one of the CFRP systems showed a 35\% reduction in load carrying capacity compared to the control specimen. The second CFRP system showed a 15\% reduction. The reduction in load carrying capacity in the case of GFRP was about 38\%. They observed that the samples exposed to condition I showed local degradation and voids as well as plasticization. Based on their observations on peeled FRP laminates, GFRP systems were observed to have deteriorated more than the CFRP laminates. The maximum degradation was observed in cases of exposure to sea water.

Abanilla et al. (2006 a) investigated the durability of wet layup CFRP laminates based on the results of 100 weeks of exposure to 6 different environments: immersion in deionized water at $23,37.8$ and $60^{\circ} \mathrm{C}$, immersion in salt water at $23^{\circ} \mathrm{C}$, immersion in alkali solution at $23^{\circ} \mathrm{C}$, and exposure to freeze-thaw conditions between -10 and $23{ }^{\circ} \mathrm{C}$ at the rate of one cycle per day. CFRP laminates with 2, 6, and 12 plies were fabricated using the wet layup method at a field site while actual rehabilitation was being performed in order to simulate real field situation. After fabrication in the field, all the specimens kept for 30 days in an environment maintained at a temperature of $23^{\circ} \mathrm{C}$ and $30 \%$ relative humidity. They concluded that although deterioration of matrix and interface decreases laminate strength significantly, it has less effect on the modulus. They correlated these mechanical properties changes to the moisture uptake, which was measured based on the increase in weight over a period of time. Based on their observations, deterioration in tensile properties increases with the number of laminate plies, while flexural properties decrease as the number of plies increases.

Karbhari and Abanilla (2007) studied the durability of unidirectional wet lay-up carbon/epoxy laminates with 2, 6 and 12 plies. Their experiment showed that by increasing the number of reinforcing layers significantly, deterioration rates increase as a result of the increasing number of resin dominated interlayer interfaces.

Using the experimental data obtained over a 3 year period of immersing the specimens in water at $23^{\circ} \mathrm{C}$, they developed prediction equations for tensile strength, flexural strength, shortbeam shear strength, and in-plane shear strength using two popular predictive approaches: the 
Arrhenius method, and the Phani and Bose approach were used to calculate strength and modulus, and are in the following general forms below:

$$
P(t)=\frac{P_{0}}{100}[A \ln (t)+B] ; \quad t>0
$$

Where $P(t)$ and $P_{0}$ are strength or modulus at time $t$ (in days), and time 0 (i.e. unexposed condition), respectively, and $A$ is a constant denoting degradation rate and $B$ is a material constant reflecting the early effect of post-cure progression. Karbhari and Abanilla (2007) concluded that in using the Arrhenius approach directly, the predicted response was in better agreement with experimental data compared to using Phani and Bose approach. It is worth mentioning that Chen (2007) found the Phani and Bose approach more appropriate for rods.

In some cases, they observed that during early stages of exposure significant differences were captured between the results of the two above mentioned approaches. This observation was explained by the fact that neither of the two predictive approaches takes into the account the competition between the two deterioration mechanisms: residual post-cure mechanism and moisture induced deterioration mechanism.

Wan et al.'s (2006) research to assess the effect of presence of water during CFRP application, and also after CFRP cure on the CFRP-concrete interface, observed that even for a time period as short as 8 weeks of submerging the specimens in water, the interfacial fracture energy was reduced by $85 \%$ which is quite surprising.

Ouyang and Wan (2008 a) investigated the effect of moisture on bond deterioration of CFRP plates externally bonded to modified double cantilever beams and also its effect on the interface fracture energy. In this experiment, the specimens were cured under 100\% humidity for 28 days, and then transferred to a lab with 35\% relative humidity and kept there for 10 months. At the end of this period, the concrete weight had almost entirely stabilized and no significant changes were observed in concrete weight. Three control specimens were tested seven days after bonding the CFRP plates. The remaining samples were immersed in water until being tested. Three specimens were tested after 2, 3, 4, 5, 6, 7, 8 weeks of immersion in water.

As another parameter, humidity in the interface was measured before occurring delamination. Ouyang and Wan (2008 a) developed a humidity measuring method whereby a digital hygrometer was used to measure the humidity in a closed space composed of the concrete 
fracture surface, an using aluminum tube, and a tapered silicone washer for sealing the top of the aluminum cap (Figure 2-22).

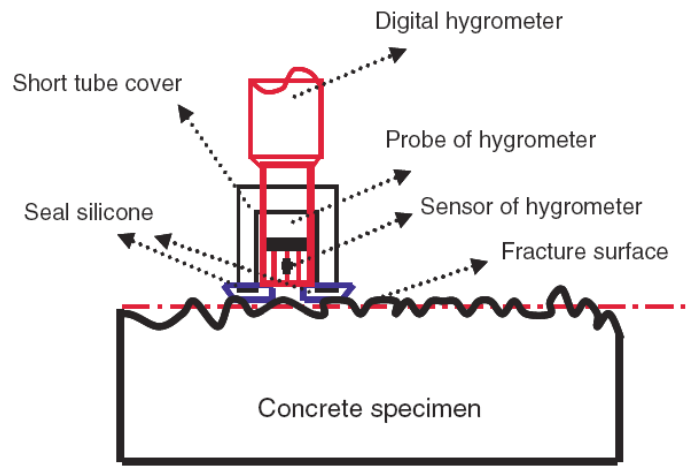

Figure 2-22 Setup to measure interface region relative humidity (Adopted from Ouyang and Wan 2008)

This closed space had no air convection in the surrounding ambient. Thus, the recorded humidity, when balanced, was considered to be the relative humidity in the FRP-concrete interface region (IRRH) before the onset of delamination.

They figured that there is a relationship between IRRH and the residual thickness of the concrete attached to the delaminated plate (RTC). The RTC was measured using a coordinate measuring machine (CMM). Based on their observations, for the IRRH greater than 55\%, fracture energy decay was accelerated. On the other hand, for the IRRH greater than 75\%, the fracture energy showed a steady trend and did not change significantly with the IRRH increase. They observed that as the immersion time and thus the moisture at the bond interface increases, the fracture surface gradually moves towards the interface resulting in a thinner layer of concrete attached to the delaminated FRP (Figure 2-23).

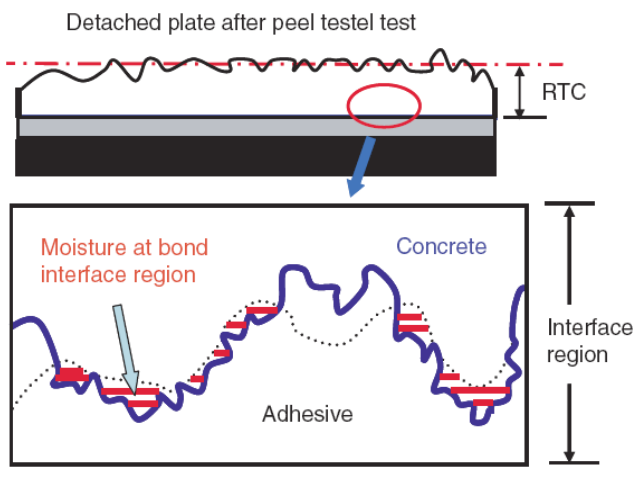

Figure 2-23 Microstructure of bond interface region between FRP and concrete (adapted from Ouyang and Wan, 2008a) 
Based on both their numerical model and experimental observations, they concluded that mode I interfacial fracture energy tends to be constant after exposure to moisture for 5 weeks.

Chen (2007) modified equation (2-35), which was suggested by Phani and Bose for strength retention of composite laminates as a way of long term prediction of GFRP bars strength retention. Assuming that GFRP bars degrade completely at infinite time of exposure, he set $\sigma_{\infty}$ (or $Y_{\infty}$ in percentage) equal to zero. He suggested that the tensile strength retention (the percentage of residual strength over original tensile strength) of GFRP bars can be calculated according to the following equation:

$$
Y=100 \exp \left(-\frac{t}{\tau}\right)
$$

Where $Y$ is the tensile strength retention (\%), $t$ is the exposure time and $\tau=\frac{1}{k}$ as expressed in the following equation:

$$
\frac{1}{k}=\frac{1}{A} \exp \left(\frac{E_{a}}{R T}\right)
$$

Equation (2-38) is another form of the Arrhenius equation.

Finally, he obtained master curves for tensile strength retention vs. exposure time at $20{ }^{\circ} \mathrm{C}$ for long-term prediction of the GFRP bars tensile strength.

As another part of his research, Chen (2007) also studied the durability of GFRP bars embedded in concrete cylinders experimentally by exposing the specimens to tap water and hot water along with thermal cycling. He used two types of concrete: normal and high performance.

The specimens were conditioned in three different environments: (1) Environment W: specimens were submerged in water in a curing tank for 90 days at room temperature; (2) Environment T: specimens were immersed in tap water for 90 days in the tanks with $60^{\circ} \mathrm{C}$ temperature; and (3) Environment E: specimens were held in environmental chambers for thermal cycles in air between $-20^{\circ}$ and $60^{\circ} \mathrm{C}$ temperatures. Based on the pull-out tests, he concluded that the bond strength and bond-slip behavior are dependent on concrete strength. He suggested that for investigating the durability of FRP-concrete bond, the extra strength that concrete gains due to the exposure to humidity should also be taken into account. He also concluded that the elevated temperature accelerates the degradation of the bond. He suggests that the degradation of the bond may be related to the degradation of the FRP material. 
Chen et al. (2006) developed a long-term prediction model for GFRP bars in concrete structures based on the Arrhenius relation, using the short-term data obtained from accelerated aging tests. The tensile strengths of the bars were measured before and after the exposure, and were considered as a measure of the durability of the specimens. GFRP bars were exposed to simulated concrete pore solutions at 20,40 , and $60^{\circ} \mathrm{C}$ for a period of 240 days. Two types of GFRP bars fabricated via commercial pultrusion process with the same diameter, $9.53 \mathrm{~mm}$, and the same resin, but different types of E-glass fibers, were used. The tensile strengths of groups of GFRP bars were measured before and after immersion in simulated pore solutions at different temperatures for different exposure times. Based on their observations, there is a dominant degradation mechanism for GFRP bars in alkaline solutions that does not appear to change with temperature or time. Based on their obtained master curves, the tensile strength retention of GFRP1 bars is predicted to drop to $50 \%$ after only a half-year exposure in Solution 1 at $20^{\circ} \mathrm{C}$, while for GFRP 2 bars exposed to Solution 2 at $20^{\circ} \mathrm{C}$, the same amount of retention was obtained after about 3 years. 


\section{Materials and Testing Program}




\subsection{Introduction}

This study consisted of testing 116 small scale concrete prisms, externally bonded, using CFRP laminates. All of the prisms had the same nominal dimensions. The same mix proportions were used for casting. CFRP reinforcements were applied to the concrete substrate in single layer form. These laminates were fabricated using the dry lay-up method. Bonding the FRP was performed according to the manufacturer's application guideline. All CFRP reinforcements had the same nominal size, and were bonded using a dry lay-up method. This chapter aims to explain the specimen and its fabrication process. In 3.2, concreting and its different aspects are discussed. Next, 3.4 discusses the bonding process and the steps should be taken prior to actual bonding including surface preparation. Finally, conditioning the samples prior to the test is explained in 3.5 .

\subsection{Concrete}

It is a well known fact that in order to obtain more accurate results regarding the CFRPconcrete interface and to define a more accurate traction-separation law, the concrete substrate should be relatively large, compared to the CFRP laminate. This is done so that the deformation of the concrete substrate would be negligible with respect to the interface. With this in mind, molds with nominal internal dimensions of length 13 in, width 5 in, and height 4 in were fabricated. For the preliminary study, the formworks were made of wood and the top surface was steel-troweled. Since quality of the surfaces sitting atop the fixture was also very important in order to assure a pure Mode II testing set up, or more accurately a test set up with Mode II dominating, some other molds were made out of steel to achieve smoother surfaces on all sides. Since fabrication and handling of wooden molds was much easier, it was decided to use the same wooden molds with Plexiglas end plates for the actual testing (Figure 3-1). Specimen details are shown in Figure 3-2. Geometrical specification of each component is shown in Table 3-1.

\subsection{Surface Preparation Importance}

Previous research (Chajes et al., 1996; Toutanji and Oritz, 2001; Lorenzis et al. 2001; Shen et al. 2002; Delaney and Karbhari 2007; Yalim et al. 2008) has shown that the concrete surface preparation can significantly affect the ultimate bond strength. Moreover, an improperly prepared surface can result in debonding or delamination of the FRP system before achieving the design load transfer (ACI 440 2R). Thus, surface preparation plays an important role in any FRP- 
concrete interface investigation, especially since failure mostly occurs a few millimeters under the FRP through concrete. "It is not easy to quantify the roughness of a concrete surface. Controlling the level and uniformity of surface roughness depends heavily on human skill and experience. This becomes critical when producing duplicate test specimens that require exactly the same roughness grades.” (Yalim et al. 2008)

Some of the most important efforts for surface preparation found in the literature are presented below:

Toutanji and Oritz (2001) compared two different ways of concrete surface preparation: water-jet and sanding. They concluded that concrete surface treatment by water jet results in superior bonding strength than surface treatment by an ordinary sander. It was also reported that in case of water-jetting, the failure load could improve about 50\% higher than sanding.

Chajes et al. (1996) investigated three different surface treatments to evaluate the effect of surface preparation on the bond quality: (1) no surface preparation (i.e., bonded to "as cast" surface); (2) grinding with a stone wheel to give a smooth finish; and (3) mechanically abrading with a wire wheel to give a finish that would leave aggregate slightly exposed (i.e., similar to sandblasting). They concluded that in order to achieve the best possible bond strength, the concrete surface should be mechanically abraded or sand blasted. They also concluded that the ultimate bond strength is proportional to the square root of the compressive strength of concrete.

De Lorenzis et al. (2001) compared the effects of two different surface preparations: Sandblasting and roughening the surface by chiseling. They observed that the specimens with surfaces prepared by chiseling failed in a much higher load level.

Yoshizawa et al. (1996) investigated surfaces roughened by either sandblasting or waterjetting. They found out that the surface treatment by water-jet increases the bond strength much more significant than that of sandblasting.

Anil and Belgin (2007) roughened the surface of the concrete beam by a mechanical grinding machine until the aggregate was exposed. They then brushed and vacuumed the surface.

Yalim et al. (2008) investigated the effect of concrete surface roughness on the bond behavior of FRP-bonded beams. Their investigation included three levels of surface roughness based on ICRI/ACI (1999); first level: CSP 1, second level: CSP 2-3, third level: CSP 6-9. They concluded that concrete surface roughness did not significantly affect the performance of FRP 
systems, whether the failure was by FRP debonding or rupture. In the end, they recommend using CSP $2-3$ as a conservative CSP for wet layup and procured FRP systems.

BASF, the FRP manufacturer, requires the surface to be prepared to a minimum profile of ICRI CSP 3. Also, according to ICRI guideline No. 03732, the risk of introducing micro-cracks when the concrete surface preparation is performed by abrasive (sand) blasting or by waterjetting is very low. According to this guideline, grinding will produce a nearly flat surface having little or no profile (middle of CSP 1 to middle of CSP 3). Abrasive (sand) blasting will provide CPS between the middle of CSP 2 to the middle of CSP 5. Unlike grinding, water-jetting will not produce a smooth and uniform surface (CSP 6 to CSP 9).

The following is brief information about how other researchers prepared the concrete surface prior to install the FRP plates or sheets.

Kamel et al. (2006) ground the concrete surface with a stone wheel and applied the epoxy primer to create a smooth flat bond surface.

Pham and Al-Mahaidi (2007) used a high pressure water-jet for surface preparation until the coarse aggregates were exposed.

Pan and Leung (2007a) used a needle-gun to roughen the surface and expose the aggregates.

Bizindavyi and Neale (1999) sandblasted the concrete surface and then cleaned it by air blasting.

Toutanji et al. (2007) used roughening and sandblasting for surface treatment and then rinsed the surface using water-jets.

Xiao et al. (2004) polished the concrete surface by means of an electric grinding wheel and then mechanically abraded the surface until the coarse aggregates could be seen.

Au and Büyüköztürk (2006) mechanically abraded the concrete surfaces to the point that sound aggregates were exposed and loosely held segregated particles were removed. The surface was then cleaned using compressed air and then by using methyl ethyl ketone (MEK) to ensure a sound adhesion surface.

El Maaddawyand Soudki (2005) sandblasted the surface and then cleaned it with a high pressure air jet.

Karbhari and Zhao (1998) abraded the concrete surface using a wire brush. 
Yalim et al. (2008) obtained CSP 1 and CSP 2-3 by grinding the surface, while CSP 6-9 were obtained by pressure washing. They used a 24 MPa capacity pressure washer and just by varying the time of exposure could get different profiles.

It logically follows that the FRP should not be bonded to a smooth concrete surface, as it may cause the FRP to slip with respect to the concrete under the applied load. As the roughness of the concrete surface increases, the bond quality increases due to more mechanical interlocking. On the other hand, the surface cannot be too rough because it may result in air voids beneath the FRP surface, which will weaken the bond.

Most of the above mentioned surface preparation methods agree upon the fact that the bond surface should be roughened somehow until the coarse aggregates are exposed. Sandblasting the concrete surface until the coarse aggregates are exposed is the most popular method for surface treatment, and as previously mentioned it is in accord with BASF requirements, since sandblasting can provide a surface finish up to CSP 5.

ICRI guideline No. 03732 defines abrasive blasting as follows: “Abrasive Blasting is used to clean and profile concrete surfaces in preparation for the application of sealers, coatings and polymer overlays.”

MBRACE, the FRP manufacturer, requires a prepared concrete surface prior to bonding which is: "structurally sound and free from contaminants such as oil, grease, curing membrane, previous coatings, dust, fungus, mass, etc.”

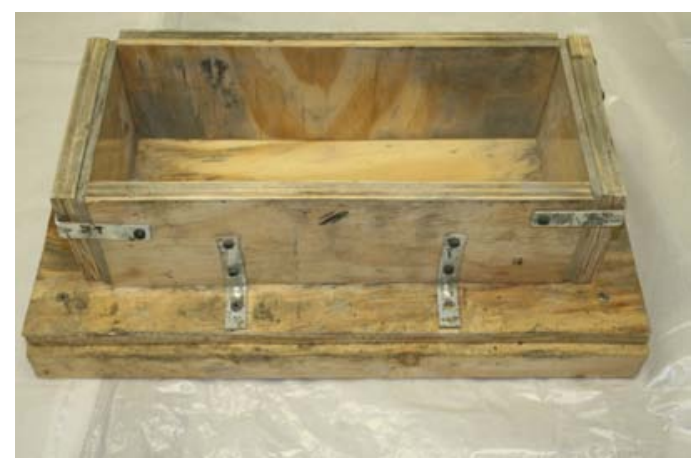




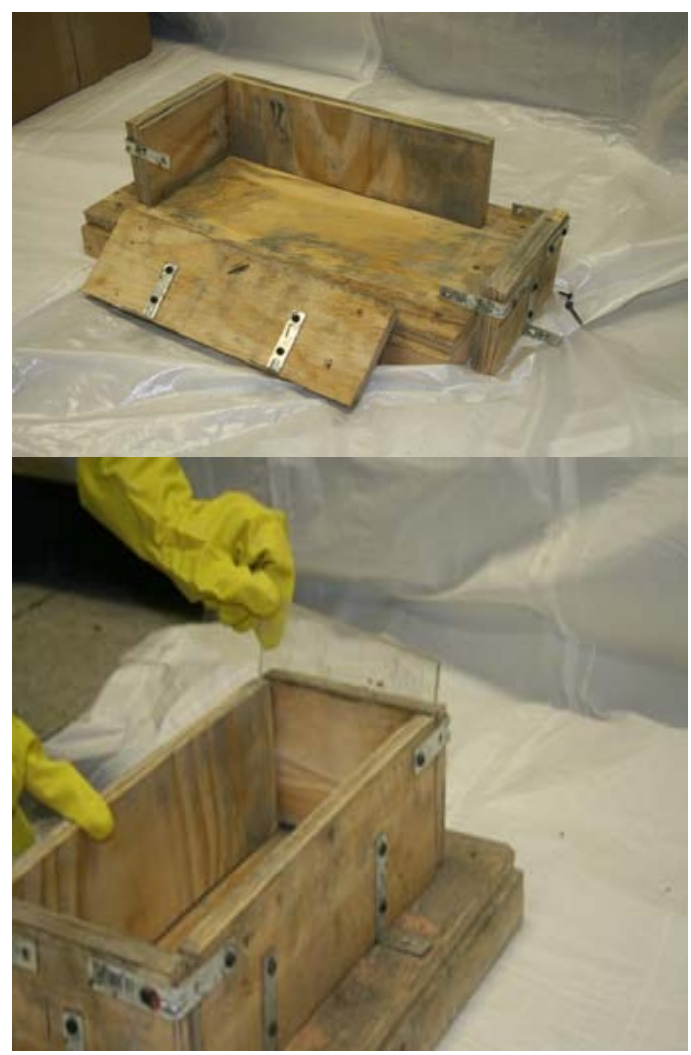

Figure 3-1 Mold

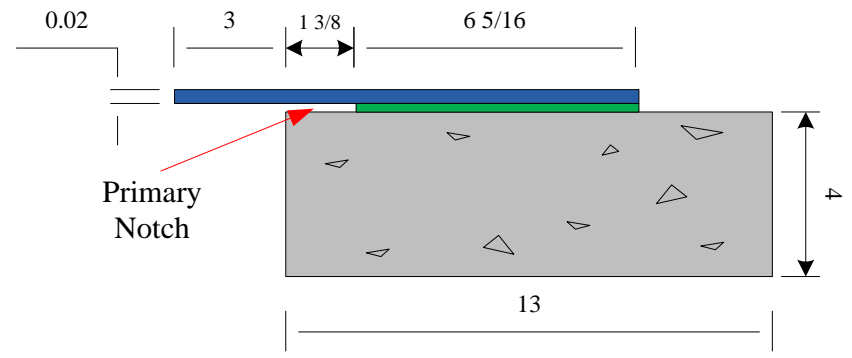

(a) Side View

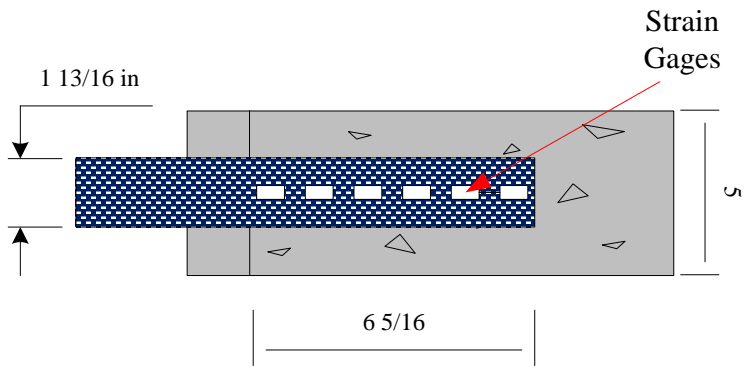

(b) Top View

Figure 3-2 Specimen Details (in)

Table 3-1 Specimen Dimensions

\begin{tabular}{l|l}
\hline \multicolumn{1}{c|}{ CoMPONENT } & \multicolumn{1}{c}{ DIMENSION } \\
\hline Concrete prism & $\begin{array}{l}13 \times 5 \times 4(\mathrm{in} \times \mathrm{in} \times \mathrm{in}) \\
(330 \times 127 \times 102(\mathrm{~mm} \times \mathrm{mm} \times \mathrm{mm}))\end{array}$ \\
\hline
\end{tabular}




\begin{tabular}{l|l}
\hline FRP thickness & $\begin{array}{l}0.02(\mathrm{in}) \\
(0.508(\mathrm{~mm}))\end{array}$ \\
\hline Bond length & $65 / 16(\mathrm{in})$ \\
& $(160(\mathrm{~m} \mathrm{~m}))$ \\
\hline FRP width & $113 / 16(\mathrm{in})$ \\
& $(46(\mathrm{~mm}))$ \\
\hline Free length of FRP & $\begin{array}{l}4(\mathrm{in}) \\
(101.6(\mathrm{~mm}))\end{array}$ \\
\hline
\end{tabular}

As previously discussed in 1.3, normal concrete was used for all specimens throughout the study. In order to obtain better workability, $800 \mathrm{~mL} / \mathrm{yd}^{3}$ of air entraining admixture (MB-AE ${ }^{\mathrm{TM}}$ 90) was also added to the mix. Air entraining admixture was added to the mix in order to obtain a more cohesive concrete without segregation. Moreover, given the climate situation in North America, it is a normal practice to use air-entraining admixtures to increase the concrete resistance with respect to freezing and thawing. ASTM Type I cement was used. Concrete mix proportions with respect to cement are shown in Table 3-2.

Table 3-2: Concrete Mix Proportions by weight

\begin{tabular}{l|l}
\hline \multicolumn{1}{c|}{ Material } & $\begin{array}{c}\text { Material/CEMENT } \\
\text { RATIO }\end{array}$ \\
\hline Coarse Aggregate & 2.5 \\
\hline Sand & 1.82 \\
\hline Water & 0.5 \\
\hline Air Entraining Admixture & $800 \mathrm{~mL} / \mathrm{yd}^{3}$ \\
\hline
\end{tabular}

Concrete was mixed using a $3 \mathrm{ft}^{3}$ capacity mixer. In order to give appropriate time interval for testing, the prisms were casted in 7 days within 45 days. Due to the limitations of the mixer capacity, 2 batches of concrete were poured each day. 30 specimens were casted per day, making a total of 150 specimens. Along with each batch of concrete, four $4 " \times 8$ " cylinders were casted and broken in the 28-days age of concrete to determine the mechanical properties of hardened concrete. The average 28-day compressive strength of the concrete determined per ASTM-C39 was 4500 psi (31 MPa). 
Mixing the concrete was performed based on ASTM C 192 standard. The detailed sequences of mixing the concrete are as follows:

1. Wetting the mixer;

2. Batching the mixing components by weight;

3. Adding the AEA to the sand and stirring well;

4. Adding $3 / 4$ of the mixing water and coarse aggregate to the mixer and mixing them for 1 $\min$;

5. Adding fine aggregate and premixed AEA, and mix for another minute;

6. Adding cement and about $90 \%$ of the remaining water, and mixing for about 2-3 minutes.

7. Stopping the mixer letting the concrete rest for a minute, and mixing for 2 more minutes.

MB-AE ${ }^{\mathrm{TM}} 90$ was used as air-entraining admixture. The amount of the admixture recommended by MB-AE ${ }^{\mathrm{TM}} 90$ data sheet is $16-260 \mathrm{~mL} / 100 \mathrm{~kg}$ of cementitious material.

A water-based mold releasing agent, Rheofinish 211, was used to coat inside the mold before pouring concrete in order to reduce the surface imperfections which might adversely affect the bond properties and obtain a better surface finish. Based on the manufacturer's recommendation, the Rehofinish was applied using a spray to the interior side of the mold. (Figure 3-3)

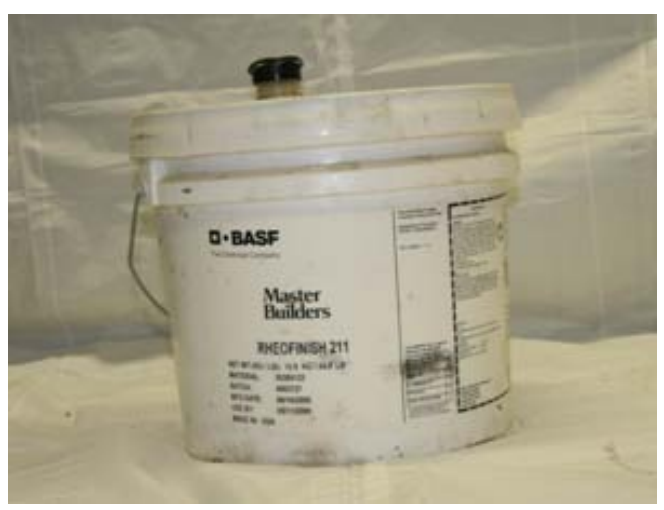

Figure 3-3 Water-Based Mold releasing Agent

The concrete was placed in the forms in three layers, each layer followed by compacting the fresh concrete by rodding it 12 times and consolidating by tapping the sides of the mold a couple of times, in order to reduce the amount of entrapped air and stone pockets.

After filling up the molds, the exposed surface was steel-trowelled to yield a smooth finish. As the exposed side of the prism was supposed to sit against the fixture, its smoothness was of 
great importance. It is also important for cylinder specimens because a rough surface can cause stress concentrations in the compression test and give invalid values for the compressive strength.

After casting, specimens were covered with wet burlap and a thick plastic sheet layer in order to maintain moisture. Specimens were demolded after 24 hours and immersed in lime saturated water for 28 days to be cured. After curing, cylinders were loaded under compression until failure per ASTM-C39/C 39M - 05 to determine the batch's average 28-day compressive strength. In case the ends of the cylinders were not flat, the two ends were grinded using a manual grinder before running the compression test to ensure a relatively flat surface and minimize the local variation effects. During the compression test, both ends of the cylinder were constrained by steel caps cushioned with neoprene pads of about $5 \mathrm{~mm}$ thickness. The reason for using these pads is to obtain a better approximation of the uniaxial compressive strength due to allowing the concrete to expand with very little constraint (Mindess et al., 2003). The results, along with standard deviation and the coefficient of variance for all the batches are presented in Table 3-3 and Table 3-4. At the same time, the prisms were rinsed with water to clean up the surface from the lime sediments and then transferred to a temperature and humidity-controlled chamber at $25^{\circ} \mathrm{C}\left(77^{\circ} \mathrm{F}\right)$ and $50 \% \mathrm{RH}$, to dry until 90 days to minimize the shrinkage effect (See Figure 3-4).

Table 3-3 Average 28-day Copmressive Strength, Standard Deviation, and Coefficient of Variance

\begin{tabular}{c|c|c|c}
\hline & $\begin{array}{c}\text { Mean } \text { f'c }^{\prime} \\
\text { (psi) }\end{array}$ & STD (psi) & COV \\
\hline Batch 1 & 4682 & 338.40 & 13.84 \\
Batch 2 & 4761 & 421.71 & 11.29 \\
Batch 3 & 4907 & 358.83 & 13.68 \\
Batch 4 & 4271 & 438.28 & 9.74 \\
Batch 5 & 4324 & 358.83 & 12.05 \\
Batch 6 & 4456 & 421.08 & 10.58 \\
Batch 7 & 4297 & 443.07 & 9.70 \\
Batch 8 & 4271 & 279.47 & 15.28 \\
Batch 9 & 4947 & 629.53 & 7.86 \\
Batch 10 & 4363 & 343.05 & 12.72 \\
Batch 11 & 4509 & 243.11 & 18.55 \\
Batch 12 & 4112 & 376.07 & 10.93
\end{tabular}




\begin{tabular}{l|l|l|l} 
Batch 13 & 4470 & 338.40 & 13.21 \\
Batch 14 & 4589 & 400.53 & 11.46 \\
\hline
\end{tabular}

Table 3-4 Average 28-day Compressive Strength, STD, and COV of all Batches

\begin{tabular}{c|c|c}
\hline $\begin{array}{c}\text { Mean } \boldsymbol{f}^{\prime}{ }_{c} \\
(\mathbf{p s i})\end{array}$ & STD (psi) & COV \\
\hline $\begin{array}{c}4500 \\
(31 \mathrm{MPa})\end{array}$ & 252 & 17.85 \\
\hline
\end{tabular}

$2712^{\prime} \times 6^{\prime \prime} \times 6^{\prime \prime}$ concrete beams were also casted using the same batch to measure the FRPconcrete pull-off strength as well as the concrete surface tensile strength. Steel molds were used.

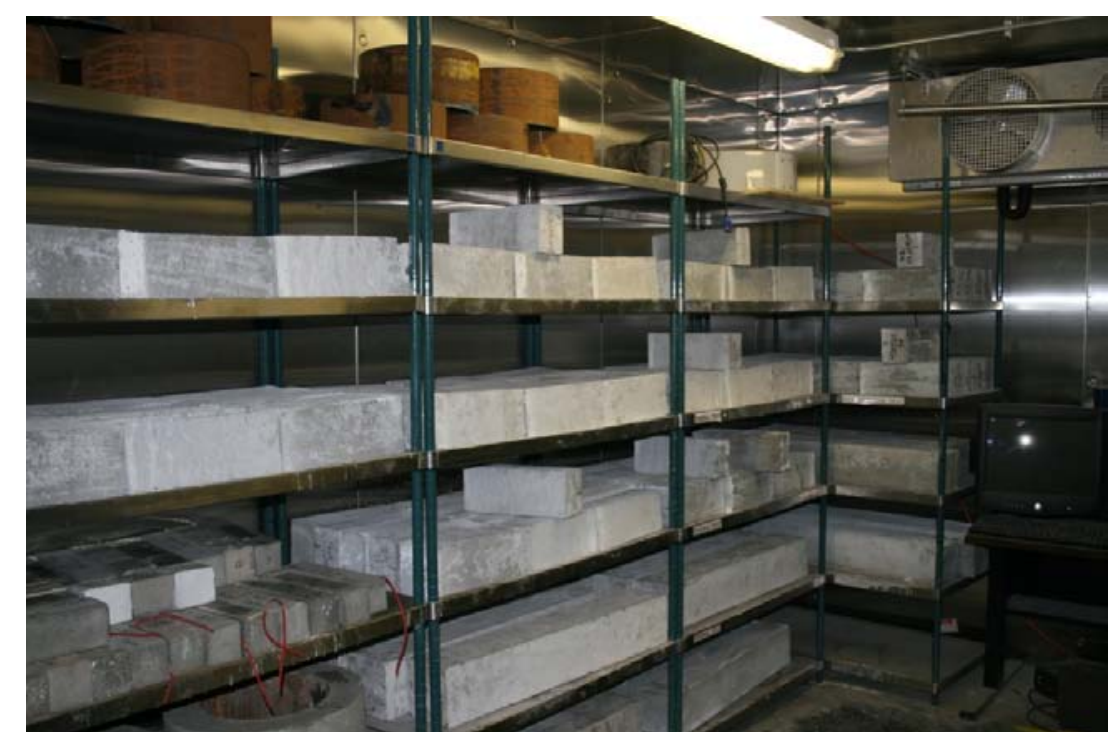

Figure 3-4 temperature and humidity-controlled chamber

The concrete slump was $8 \frac{1}{1} 2$ in which indicates a pretty workable fresh concrete. The air content of the fresh concrete was 6\% as measured using the pressure method per ASTM C 231. 


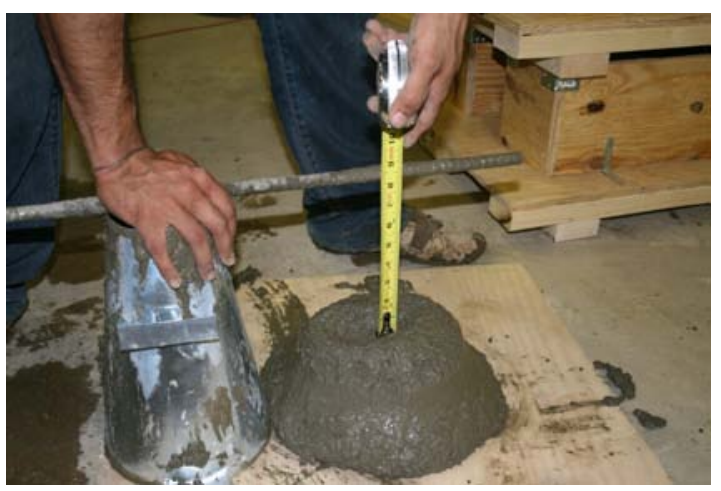

Figure 3-5 Slump Test

\subsection{Bonding}

\subsubsection{CFRP System}

For composite sheets, unidirectional MBrace CF130 from BASF was used. The fabric was cut into strips of $1113 / 16 \times 113 / 16$ in and with a bond length of $65 / 16$, making a $11 / 2$ in primary notch, and a 4 in free length, in order to be gripped into the MTS machine.

It will be shown in section 4.7.3 that for calculating slip in order to obtain the $\tau$ - $\delta$ curves, it is assumed that the gage farthest from the loaded end is perfectly bonded to the FRP; in other words, $s\left(x_{n}\right)=0$. For this assumption to be valid, the bond length must be sufficiently long compared to the effective bond length. Since according to the literature the effective bond length for FRP sheets ranges between 2.4 to 4.3 in (60 to $110 \mathrm{~mm}$ ), (Chajes et al. 1996; Maeda et al. 1997; Ali-Ahmad et al. 2005; Kamel et al. 2006) the bond length in this study is taken as 6 5/16 in $(160 \mathrm{~mm})$ to be sufficiently long making the aforementioned assumption correct.

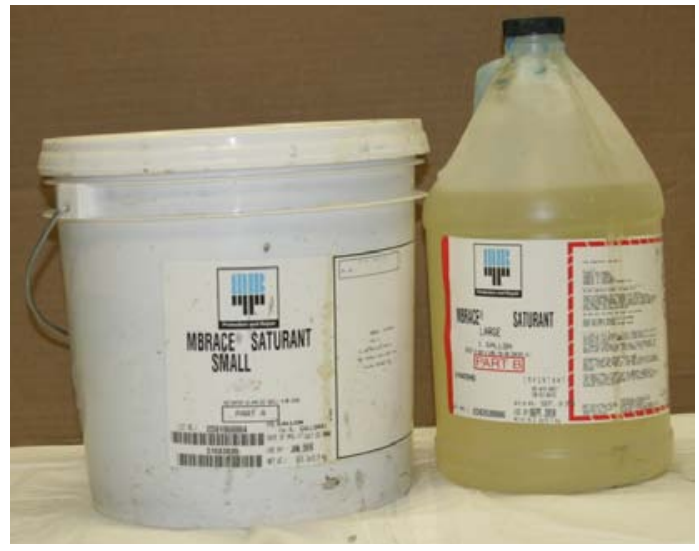




\section{Figure 3-6 CFRP System}

The adhesive system was also MBrace consisting of two-part primer and two-part saturant (Figure 3-6). Both saturant and primer were epoxy resins, but they are different in terms of application purposes and appearance.

The primer, which has amber color, is applied to the concrete substrate after surface preparation to fill the pores in order to provide a more appropriate bond. In this study, the primer served for another purpose as well; Except for the interface, the other sides of the concrete prism were also coated by the primer to ensure that the water penetration is only through the interface. The saturant, on the other hand, has an opaque blue color and impregnates the fibers to create the composite and works as the glue to bond CFRP to the concrete substrate. Material properties of the fabrics, the epoxy are presented in Table 3-5and Table 3-6, respectively.

Table 3-5 Physical Properties of MBrace CF130 (Carbon Fiber Fabrics)

\begin{tabular}{l|l}
\hline \multicolumn{1}{c|}{ PROPERTY } & \multicolumn{1}{c}{ VALUE } \\
\hline Area Density & $300 \mathrm{~g} \cdot \mathrm{m} 2$ \\
\hline Effective thickness & $0.165 \mathrm{~mm}$ \\
\hline $\begin{array}{l}\text { Tensile Modulus of elasticity, } \\
\text { ASTM D3039 }\end{array}$ & $230 \mathrm{GPa}$ \\
\hline $\begin{array}{l}\text { Ultimate deformation (ASTM } \\
\text { D3039) }\end{array}$ & $1.5 \%$ \\
\hline Tensile Strength, ASTM D3039 & $3,000 \mathrm{MPa}$ \\
\hline Color & Black \\
\hline
\end{tabular}

Table 3-6 MBrace Epoxy Resin Specifications

\begin{tabular}{l|l}
\hline \multicolumn{1}{c|}{ ProperTY } & \multicolumn{1}{c}{ VALUE } \\
\hline $\begin{array}{l}\text { Bonding to concrete (direct } \\
\text { traction), pr EN 1542 }\end{array}$ & $\begin{array}{l}\text { > 3.5 MPa } \\
\text { (concrete failure) }\end{array}$ \\
\hline Tensile Strength ASTM D638 & $>50 \mathrm{MPa}$ \\
\hline $\begin{array}{l}\text { Modulus of elasticity, ASTM } \\
\text { D638 }\end{array}$ & $>3000 \mathrm{MPa}$ \\
\hline Percent Elongation & $2.5 \%$ \\
\hline Flexural Strength ASTM D790 & $>120 \mathrm{MPa}$ \\
\hline Flexural Modulus ASTM D790 & $>3500 \mathrm{MPa}$ \\
\hline $\begin{array}{l}\text { Compressive strength ASTM } \\
\text { D695 }\end{array}$ & $>80 \mathrm{MPa}$ \\
\hline Specific gravity & 1.12 \\
\hline Viscosity & 1600 Centipoises
\end{tabular}


According to the technical data provided by the manufacturer via direct correspondence, the average thickness of a single ply laminate is 0.02 in, and its average tensile modulus of elasticity is $10,725,000$ psi. It should be noted that the single ply laminate used for repairing the bridges is essentially field fabricated. Therefore, the actual values of the mechanical properties can vary quite significantly, depending on the quantity of resin used, the presence of moisture during placing or curing, the proficiency of the operator, and other such variables. The above values were provided by the manufacturer upon our request via correspondence rather than a technical data sheet.

\subsubsection{Surface Preparation}

After a thorough literature review on different methods of surface preparation (Section 3.3), sandblasting was chosen to treat the concrete surface before bonding the CFRP. For the preliminary study, a portable sandblaster was used. The process proved to be a cumbersome, messy, and time consuming. Due to the amount of the dust created in using the portable blaster whereby dust travels all over the place, it was decided that the sandblasting had to be done in an open area. A large piece of thick plastic sheet was spread on the floor to reduce the environment pollution. The samples were placed in a heavy duty container to collect the excess sand to be reused. Even in an open area and using the plastic sheet and the container clean up and dust collection was not completely feasible and this created an environmental hazard. As the sandblasting had to be done in an open area, weather conditions created more limitations. It was not possible to treat the samples in rainy or snowy days. Plus, wearing all the protective clothes and the respirator in sunny days made the work quite uncomfortable. Moreover, blasting even one specimen was a pretty time consuming, about 45 minutes per sample. Recollecting the beads for reuse was not easily done also created lots of waste. As the glass beads and especially dust particles were quite small, even protective clothes were not really effective; thus sandblasting too many samples using the portable blaster could be harmful for the operator's health. Given all these difficulties, it was decided to use a blast cabinet working with the air pressure instead of a portable blaster.

The Blasting cabinet (Figure 3-7), on the other hand, does not have any of these problems. It has appropriate lighting inside and the operator is not exposed to the dust during the operation. It 
also has a dust collector in the bottom which not only keeps the environment clean, but also allows reusing the beads over and over until they are all crushed. The most important factor is that as all the dust is remained in the cabinet, there is no need to a respirator. It also has two gloves in which the operator places his/her hands to blast the specimens inside the cabinet. Thus, no special clothing is required to protect the operator.

The inside dimensions of the blast cabinet was $36 \times 48 \times 36 \mathrm{in}^{3}$. The Ballotini D5070 glass beads with maximum nominal diameter of $212 \mu$ (US sieve 50-70) were chosen as the blasting media. Although in case of using aluminum oxide beads, the blasting process would be much faster but aluminum oxide beads could cause microcracks which would have adversely affect the bond behavior.

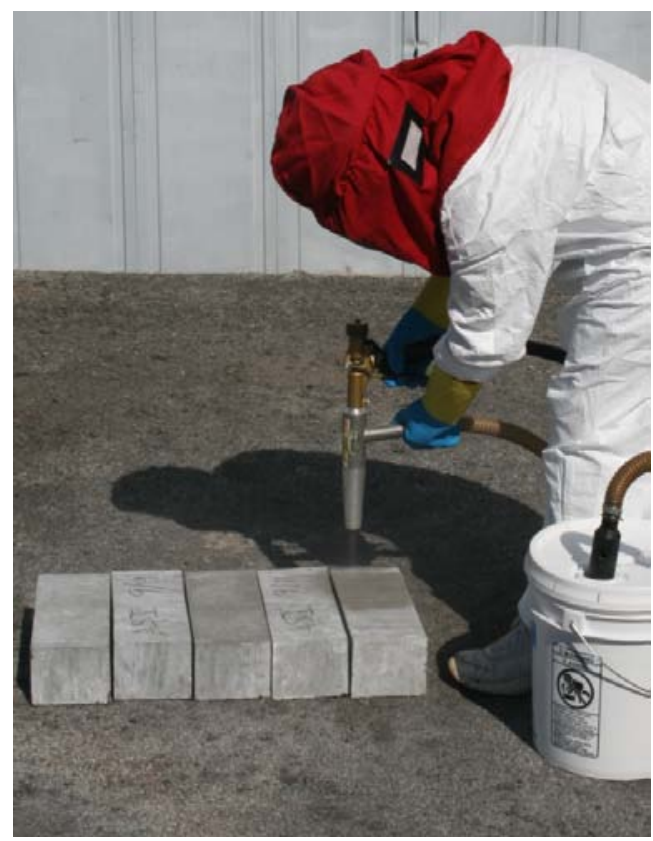

(a)

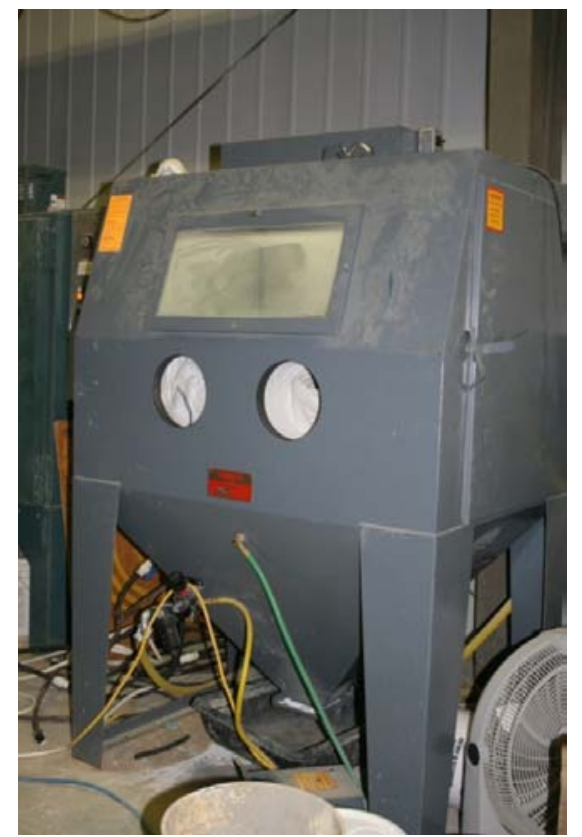

(b)

Figure 3-7 (a) Portable Sandblasting, (b) Sandblasting Cabinet

During the blasting, the treated surfaces were compared to the ICRI chips by visual inspection to make sure coarse aggregates were exposed and a CSP3-4 was achieved.

Although 48 specimens can be fit into the cabinet, only four samples were blasted at one time to make sure that all surfaces are treated in the same way.

A pressurized air-hose was used to remove the dust and the particles from the glass beads left on the prisms. Finally, the locations of where the CFRP sheets were going to be boned were marked on the beams using a permanent marker. 


\subsubsection{Priming}

Before initiating the priming process, a thick layer of plastic sheeting was spread on the floor to protect the lab flooring from any potential messy spills. The prisms were placed on the plastic sheet with enough space between the specimens so that one can easily move in between them and have access to all the sides. As the pot life of the primer was 30 minutes and would have got hardened after 24 hours, the most economical regular paint brushes were used and disposed after 45 minutes of use.

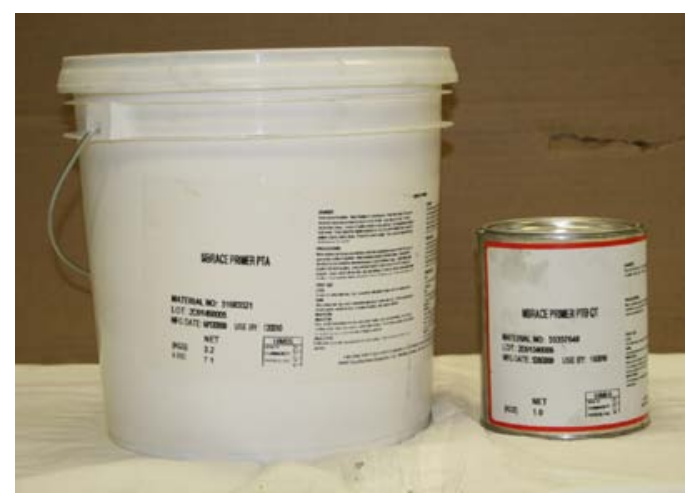

Figure 3-8 Two-Part Primer

As discussed in 3.4.1, the primer consisted of two parts: Part A, and Part B (Figure 3-8). The mix ratio by volume was $A / B=3 / 1$, as recommended by the manufacturer. The average primer used to coat 30 specimens was a liter. It worth mentioning that as the interface properties to some extent depends on the thickness of the epoxy. The same amount of epoxy, i.e., one liter, was used for all the batches.

The primer pot life was suggested 35 minutes at $23^{\circ} \mathrm{C}$ and 25 minutes at $32^{\circ} \mathrm{C}$. The pot life can vary based upon the ambient temperature of where the epoxies are stored, as well as the ambient temperature and humidity of where they are applied. It also depends on the quantity of the mix being used. In order to mix the primer, a drill with an appropriate mixing paddle was used. The two parts were mixed at a low speed for 3 minutes, as recommended by the manufacturer.

\subsubsection{Saturating}

In order to introduce the predefined notch at the end of the specimen between FRP and concrete, a small piece of wax paper was placed in that location prior to bonding. The wax paper was fixed in its place using duct tape (Figure 3-9). 


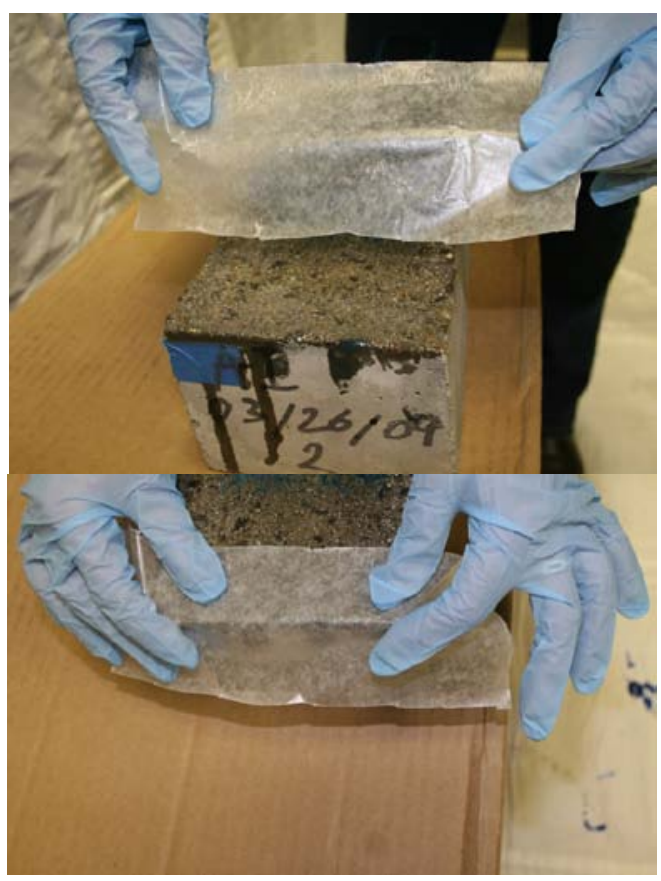

Figure 3-9 Wax Paper

Just as with the primer, the saturant also comes in two parts as well: part A and part B. The mix ratio is also the same: $\mathrm{A} / \mathrm{B}=3 / 1$ by volume. Prior to mixing the two parts, part $\mathrm{A}$ alone should be premixed using a low speed drill with appropriate paddler for 3 minutes. The combined components then should be mixed for another 3 minutes using a new paddler. It is important not to use the same paddler as had been used to mix part $\mathrm{A}$, as it can change the ratio of the two components towards more volume of Part A. This is of course of less importance in case of mixing larger amounts of the saturant. The primer and saturant mix ration and pot life are presented in Table 3-7.

Table 3-7 Color, Mix Ratio, and Pot Life of the MBrace Epoxy Resins

\begin{tabular}{l|l|l}
\hline & \multicolumn{1}{|c|}{ Primer } & \multicolumn{1}{c}{ SATURANT } \\
\hline $\begin{array}{l}\text { Color } \\
\text { Part A } \\
\begin{array}{l}\text { Part B } \\
\text { Mixed }\end{array}\end{array}$ & $\begin{array}{l}\text { Amber } \\
\text { Transparent } \\
\text { Amber }\end{array}$ & $\begin{array}{l}\text { Opaque Blue } \\
\text { Transparent } \\
\text { Opaque Blue }\end{array}$ \\
\hline $\begin{array}{l}\text { Mix Ratio A/B } \\
\text { (By Volume) }\end{array}$ & $3 / 1$ & $3 / 1$ \\
\hline Pot life at $23^{\circ} \mathrm{C}$ & $35 \mathrm{~min}$ & $30 \mathrm{~min}$ \\
\hline
\end{tabular}


As the CFRP was supposed to extend beyond the length of the concrete prism, wooden supports were fabricated with the same height as the concrete prisms, so that the CFRP extension can lay atop. To avoid bonding the FRP to the wood, the support was covered with a thick plastic sheet. These supports were placed in front of the prisms prior to saturation (See Figure 3-10).

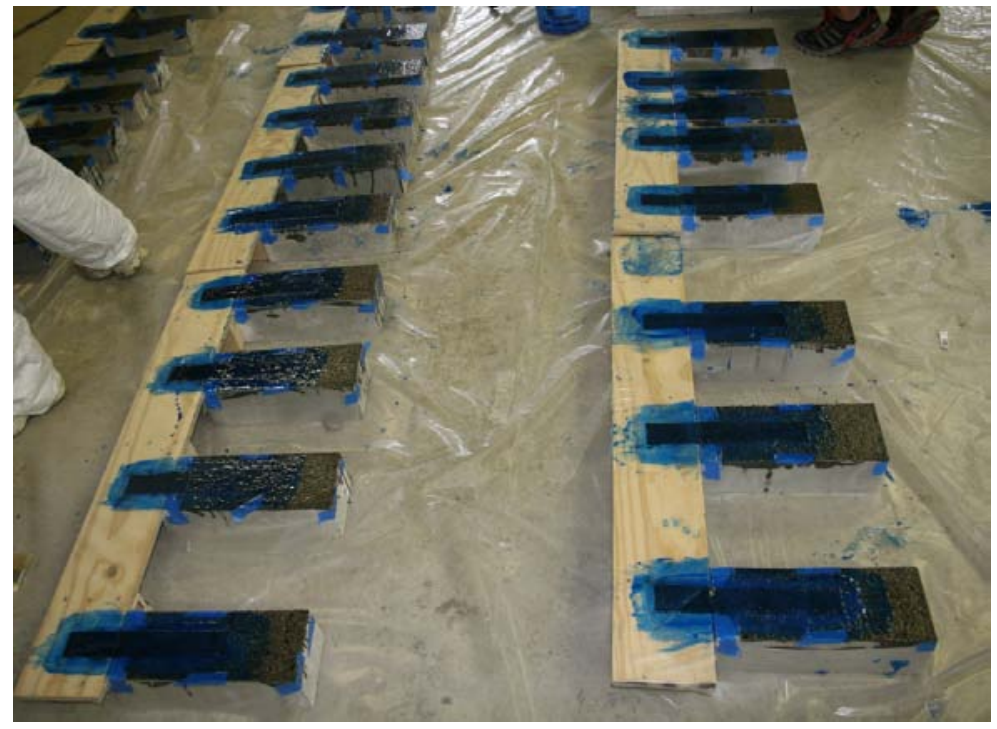

Figure 3-10 Wooden Supports

To begin the dry lay-up bonding process, while the primer was still tacky, approximately 24 hours after priming, a thin layer of the saturant was painted onto the specified area of concrete, using a paint roller. The area where the extension of the CFRP was going to be placed atop the wooden support was also painted. This was repeated twice to ensure the entire area is completely covered. Regular paint roller was selected for easy disposal after the pot life of the saturant which was about 30 minutes.

While the saturant was still wet, dry fabric was placed onto the substrate, in the marked up location and pressed by means of a squeegee and hand pressure in the fiber direction. Using a paint roller, the composite was rolled, starting from the middle of the sheet in order to push out all air bubbles, ensuring that the fabric was completely impregnated. Rolling was continued until visible signs of saturant bleeding through the fabric were visualized. After removal of all air bubbles from the fabric, a second layer of saturant was applied using a paint roller along the fiber direction. Any excess saturant was removed from the system using a squeegee. After placement, the FRP system was allowed to cure for 7 days at $23^{\circ} \mathrm{C}$ according to the manufacturers' recommendations (Installation Procedure 2006). 


\subsection{Conditioning}

Accelerated test methods have been widely used in the literature to study the durability of composites and concrete materials because using these methods the degradation of the material hastens in a less exposure duration. The maximum temperature is taken as $60^{\circ} \mathrm{C}$ based on literature which is below the $T_{g}$ of the epoxy.

At the end of the curing period, the specimens were immersed in de-ionized water in four custom designed temperature controlled tanks with 380 liter capacity until tested. Each of these double walled tanks was made of one piece molded high density polyethylene with $0.6 \mathrm{~m}$ diameter and $1.2 \mathrm{~m}$ overall height. Each tank includes a Teflon coated immersion heater (500 watts $120 \mathrm{~V}$ ) with a digital temperature control with $\pm 1^{\circ} F$ accuracy. The controls have a programmable high set point. The water temperatures of these four tanks were set as follows: $\mathrm{T}_{1}=25^{\circ} \mathrm{C}\left(77^{\circ} \mathrm{F}\right), \mathrm{T}_{2}=36^{\circ} \mathrm{C}\left(96.8^{\circ} \mathrm{F}\right), \mathrm{T}_{3}=48^{\circ} \mathrm{C}\left(118.4^{\circ} \mathrm{F}\right)$ and $\mathrm{T}_{4}=60^{\circ} \mathrm{C}\left(140^{\circ} \mathrm{F}\right)$.

The deionized water was provided by the National Research Center for Coal and Energy (NRCCE). This water was tested to find the possible ions. Table 3-8 shows the ions found in the water sample.

Table 3-8 Ions found in the deionized water

\begin{tabular}{lllllllll}
\hline Ion & Al & Ca & Fe & Mg & Mn & Na & K & Cl \\
\hline Quantity (mg/L) $<0.1$ & 0.50 & 1.32 & 0.18 & $<0.1$ & 5.00 & $<0.1$ & 0.11 \\
\hline
\end{tabular}

A rope was wrapped around the specimens loose enough so one can easily grab the specimen using a bent \# 3 rebar handle and take them out of the tank gently without affecting the FRP extension. 


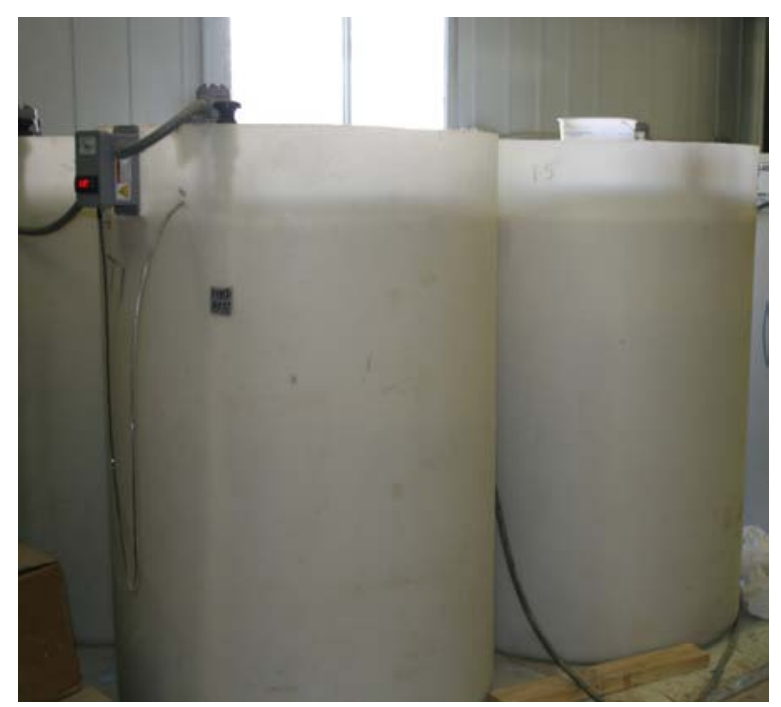

(a)

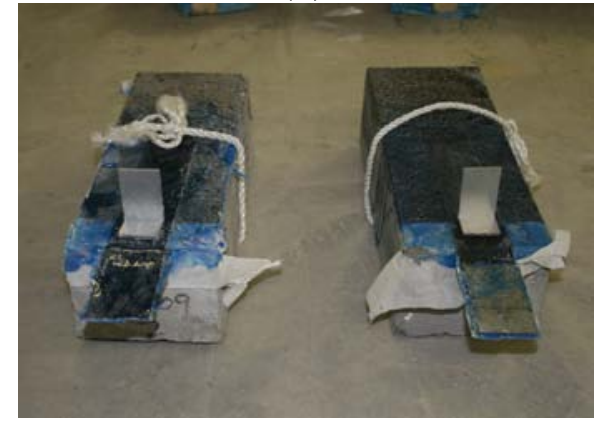

(b)

Figure 3-11 (a) Conditioning Tanks, (b) wrapping the rope around the specimens

\subsection{Discussion}

Whether an experimental study is successful to a large extent depends on the quality of the fabricated specimens and how similar the specimens are. There is always a percentage of human error involved in any experimental work, especially in the fabrication level. When it comes to concrete, it is not possible to find even two specimens which are exactly the same, due to high inhomogeneous nature of the concrete. The same problem exists for composite laminates, made by hand lay-up method, which is due to sensitivity of composite characteristics to parameters such as the angle of the fabrics and the amount of the resin used. In order to find out the potential mistakes which could be made during different steps of the experiment, which include mixing and pouring the concrete, surface preparation, bonding CFRP, several trial specimens were fabricated and tested. This revealed some mistakes and problems, which were later on avoided during the real test. 


\subsection{Summary}

The material in use and the specimen fabrication are fully described in this chapter. Normal concrete was used to cast prisms of $13 \times 5 \times 4$ in $\times$ in $\times$ in $(330 \times 127 \times 102 \mathrm{~mm} \times \mathrm{mm} \times \mathrm{mm})$. Carbon fibers were used to fabricate 10 5/16×1 13/16 in $\times$ in $(160 \times 45 \mathrm{~mm} \times \mathrm{mm})$ unidirectional single layer laminates. A 1 3/8 in (35 mm) notch was introduced at the loaded end of the specimen. The concrete surface was treated by sandblasting in order to achieve a CSP-4. The specimens were exposed to two combined environmental conditions: (1) immersion in deionized water varying from 0-15 weeks, (2) combined with temperatures varying from $77^{\circ} \mathrm{F}$ to $140^{\circ} \mathrm{F}\left(25^{\circ} \mathrm{C}\right.$ to $\left.60^{\circ} \mathrm{C}\right)$.

In the following chapter, testing protocol, and the data reduction methods are discussed. 


\section{Testing Method and Data Reduction}




\subsection{Introduction}

As discussed earlier in chapter 3, Mode II CFRP-concrete interface fracture testing was conducted using a single shear testing set up. The $\tau$ - $\delta$ curves were obtained using two different methods: a new J-integral method, and the traditional strain-based method. Load and slip at the tip of the notch were recorded to be used in the J-integral method; an internal load cell in the testing machine was continuously recording the load. The slip was measured using a spring loaded LVDT at the same sampling rate as the load. The strain distribution in the FRP which was used in traditional method, and was determined using several strain gages mounted on its surface. The captured strain field was used to obtain the traction-separation law, and the interfacial fracture energy by the traditional strain based approach. This chapter describes the experimental protocol as well as the data reduction method followed in this study to develop the traction-separation law of the CFRP-concrete interface.

\subsection{Fixture Fabrication}

A new fixture was designed and fabricated to run the Mode II fracture tests performed on MTS machine (Figure 4-1). Using this fixture, the peeling effect was negligible and the failure could be assumed as a pure mode II. The fixture was composed of 10 bulk parts which included:

- $\quad$ Base Plate (1 piece, Figure 4-2)

- $\quad$ Top Aluminum Plate (1 piece, Figure 4-3)

- $\quad$ Top Steel Plate (1 piece, Figure 4-4)

- Aluminum L-shaped supports (3 pieces, Figure 4-5)

- Steel threaded rods (4 pieces, Figure 4-6) 

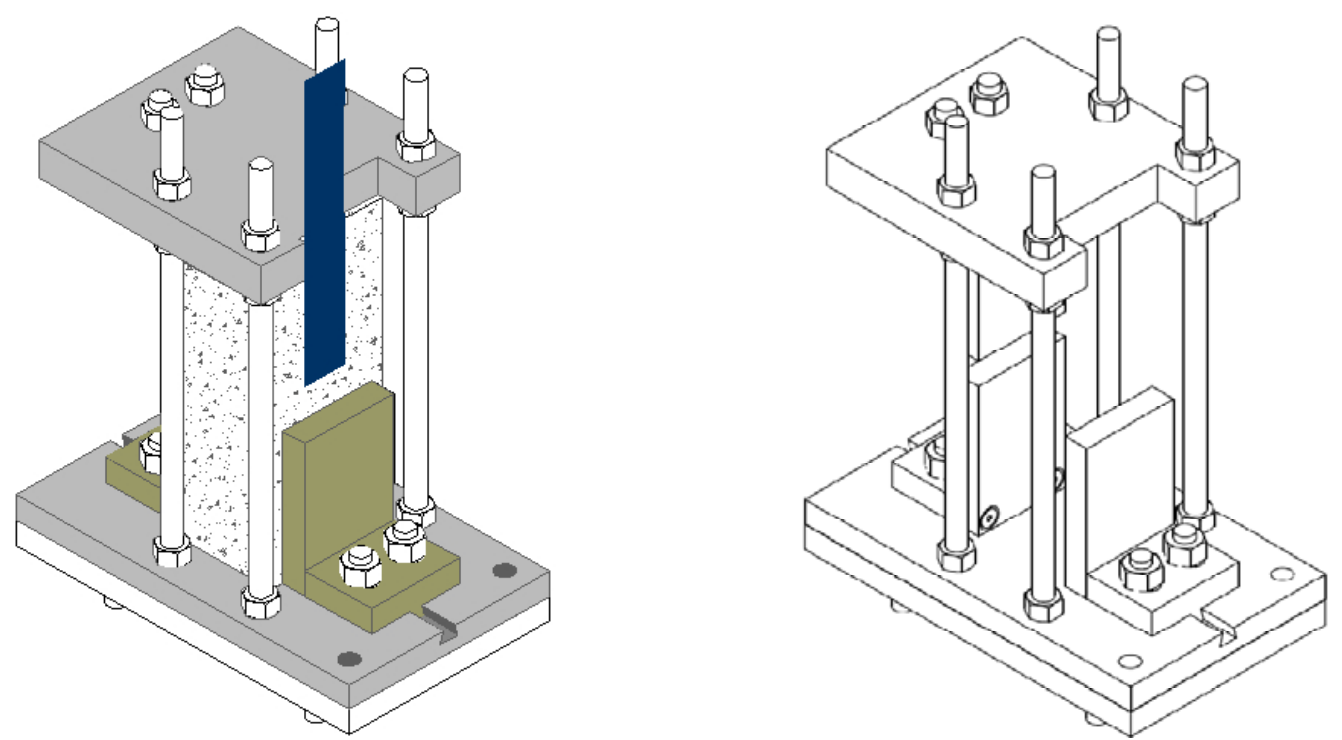

Figure 4-1 Fixture and the Specimen

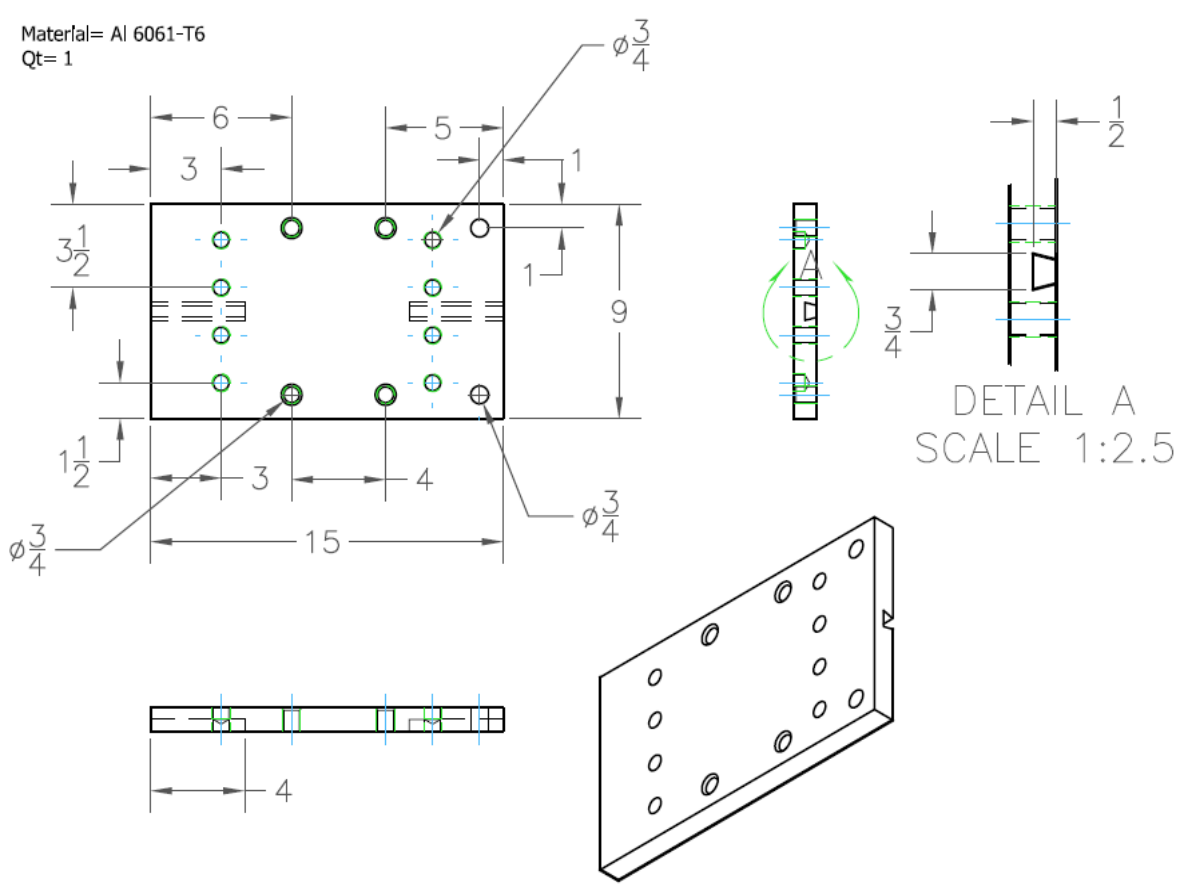

Figure 4-2 Fixture, Base Plate 


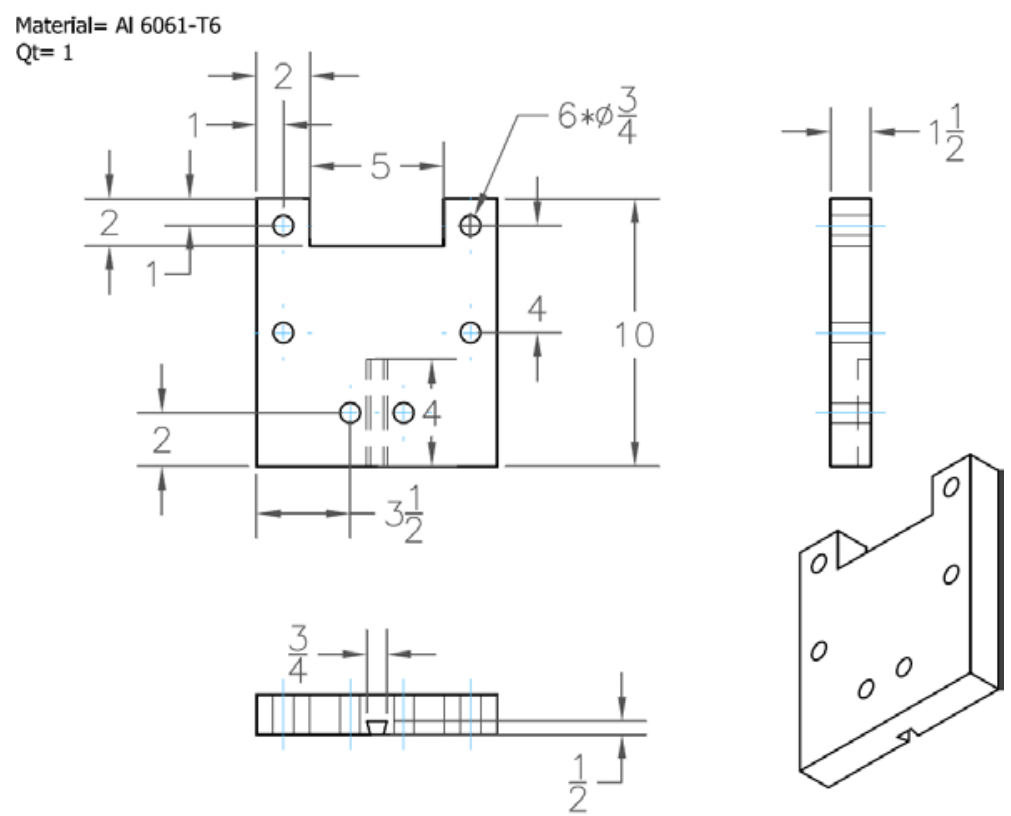

Figure 4-3 Fixture, Top Aluminum Plate

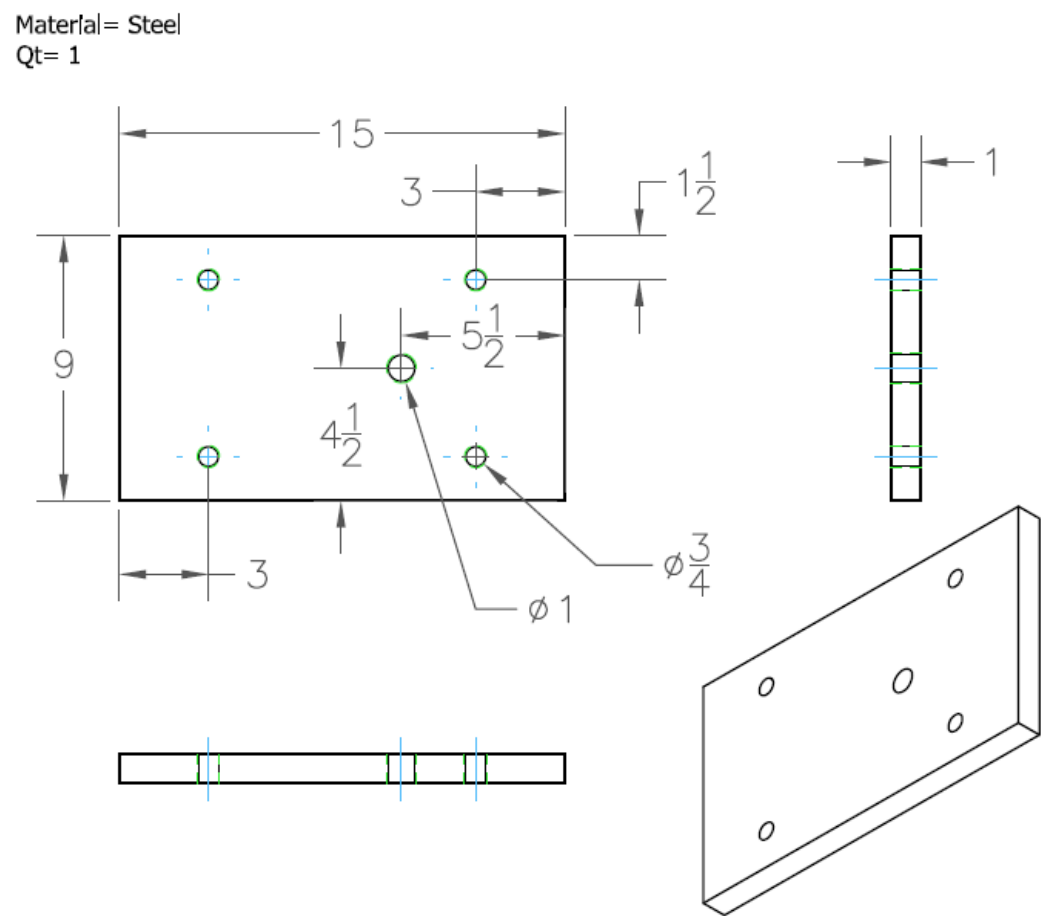

Figure 4-4 Fixture, Top Steel Plate

The top steel plate (Figure 4-4) is bolted to the upper grips of MTS machine. In order to keep the surfaces of the fixture level during all the times, another plate (the top aluminum plate) is bolted to the steel plate, and the sample is being leveled by tightening or loosening the bolts. 

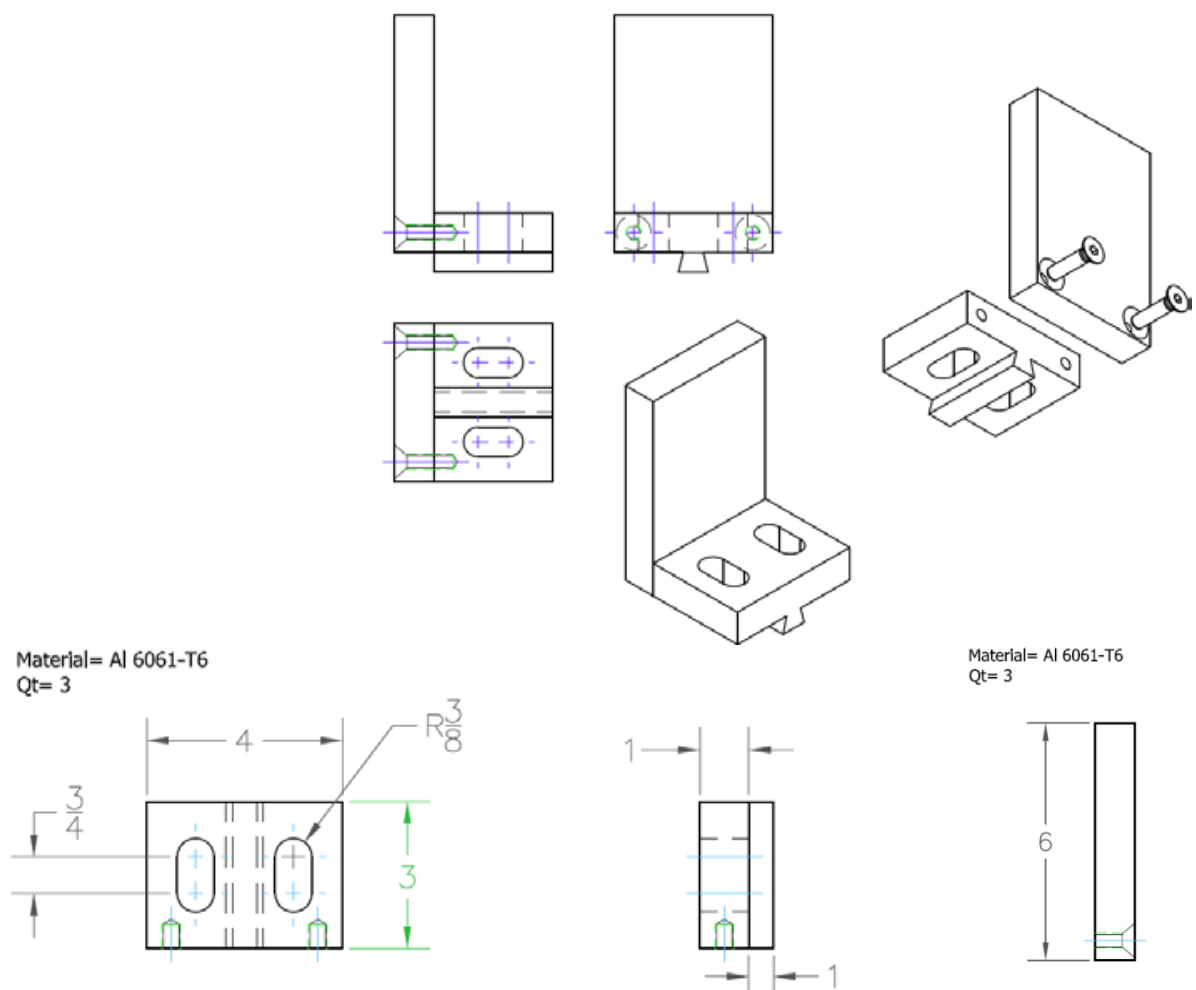

Material= Al 6061- $\mathrm{T} 6$
$\mathrm{Qt}=3$
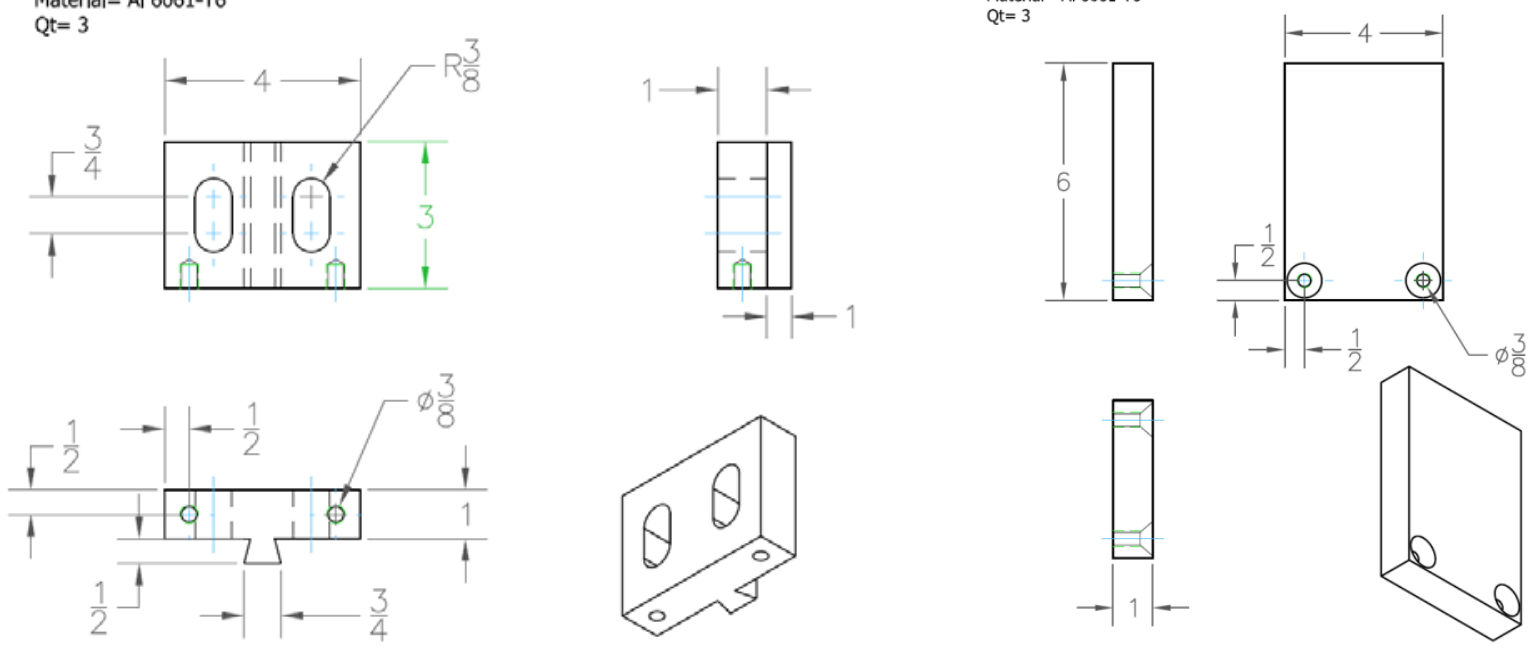

Figure 4-5 L-shaped Support

Although aluminum has been used for plates, the fixture turned out too heavy making it difficult to take it off the MTS machine every time for testing a new specimen. The grooves designed for the supports prevent the necessity of taking the fixture off, unless the machine is going to be used for some other experiment. Only by taking off the support on the base plate, one can replace the specimen easily. In this way the fixture will stay on the MTS machine throughout the experiment period. In case the plates are not level, one can adjust them only by tightening the threaded rods between the top and the bottom plates (Figure 4-6). 


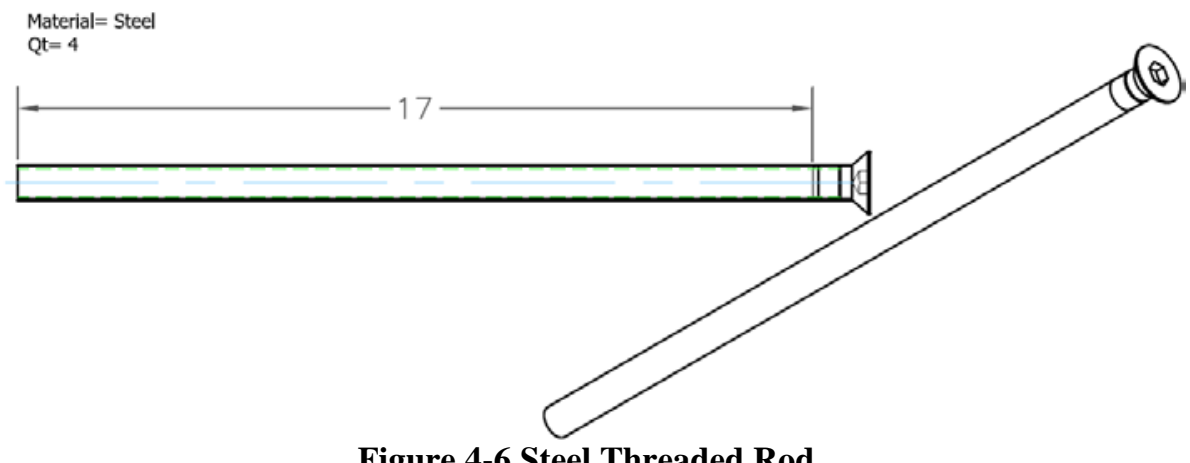

Figure 4-6 Steel Threaded Rod

\subsection{Instrumentation}

\subsubsection{Data Acquisition System}

A Vishay 5000 scanner was used for data acquisition. StrainSmart software version 4.01 was used to record all the data (load, slip via LVDT, and strain). Two strain gage cards model 5110, an LVDT card model 5140, and a high level card model 5130 were used in system 5000 to acquire strain, slip, and load, respectively. The load and the displacement of the MTS actuator (stroke) were independently recorded by the 793 Control Software which is the software controlling the MTS. However, as it is not possible to accurately correlate the two sets of data recorded using two different data acquisition systems, the load and stroke output as digital signals between -10 and $+10 \mathrm{~V}$ were hooked up to the Vishay high level card, and were recorded by only one data acquisition system (StrainSmart), and could be easily correlated together.

\subsubsection{Strain gages}

Some researchers (Taljsten 1997; de Lorenzis et al. 2001; Bizindavyi and Neale 1999, Mazzotti et al. 2004) have used strain gages mounted on the FRP surface to obtain strain field in the FRP during the load application. Subramaniam et al. (2007 a) used digital image correlation (DIC) to obtain the strain in FRP. Although DIC has the advantage of obtaining a spatial and continuous field of strain, the required equipment is not always available. Since the strain field obtained using strain gages is adequate enough to develop the traction-separation law, the strain distribution along the length of the CFRP is obtained by means of uniaxial strain gages mounted on top of the FRP layer.

After the specimens were taken out from the conditioning tank, they were allowed to dry for 24 hours, then six Vishay C2A-06-250LW-350 strain gages with 350-Ohm resistance and 1/4 in 
gage length were mounted on top of the FRP layer along its centerline starting from right on top of the notch in 1 in intervals. Figure 4-7 shows the strain gages locations.

\subsubsection{Linear Variable Displacement Transformer (LVDT)}

An RDP spring-return LVDT, model ACT 1000 A, with a range of $\pm 25.4 \mathrm{~mm}( \pm 1 \mathrm{in})$ was used to measure the slip at the beginning of the bond on the loaded-end side. An MB01 mounting block was attached to an aluminum plate which during the test was attached to the concrete prism. With this set up, the LVDT will not move with respect to the concrete prism, and the displacement measured by the LVDT will be the relative slip between the FRP and the concrete prism.

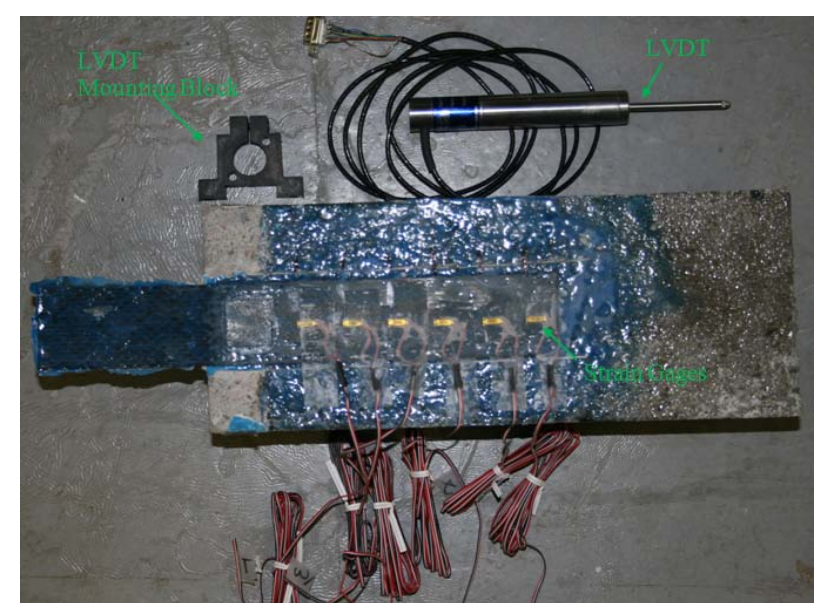

Figure 4-7 Instrumentation

Prior to running the test, an aluminum bracket was boned to the FRP layer at the exact point where bonding starts, where the notch ends. The tip of the LVDT sits on this bracket, as shown in Figure 4-7.

\subsection{Testing Procedure}

The single shear tests were conducted in an MTS machine with 20,000 lbf (98 kN) capacity. The concrete prism was placed into a custom-made rigid testing fixture (See Section 4.2). The specimen was constrained against any horizontal movement or rotation by means of the three fixture supports as shown in Figure 4-8. Vertical movement was avoided by tightening the bolts on the base plate. These bolts also served as tools to ensure the base plate were level during the test (See 4.2 for more details about the fixture). The free end of the FRP was clamped to the MTS hydraulic wedge grips \#647. The traction force was then applied to the FRP by means of 
the MTS actuator. The tests were performed under displacement control at a rate of $0.002 \mathrm{~mm} / \mathrm{s}$ up to failure. (Figure 4-8)

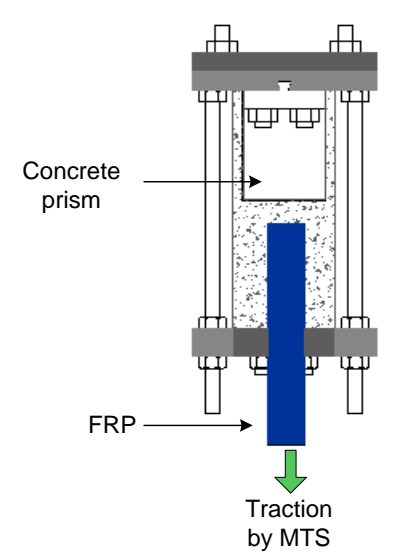

\subsection{Testing Protocol}

\subsubsection{Single Shear Tests}

A total of 116 specimens were tested under shear loading conditions. Four samples were control specimens and the others were immersed in deionized water in four custom-made temperature-controlled tanks with $\mathrm{T}_{1}=77^{\circ} \mathrm{F}\left(25^{\circ} \mathrm{C}\right), \mathrm{T}_{2}=96.8^{\circ} \mathrm{F}\left(36^{\circ} \mathrm{C}\right), \mathrm{T}_{3}=118.4^{\circ} \mathrm{F}\left(48^{\circ} \mathrm{C}\right)$, and $\mathrm{T}_{4}=140^{\circ} \mathrm{F}\left(60^{\circ} \mathrm{C}\right)$, respectively. 28 specimens for each temperature were tested throughout the test period. Starting from the end of the third week of conditioning, four samples were tested for each temperature at every two weeks until the $15^{\text {th }}$ week. Figure $4-9$ shows the testing plan in more details. 


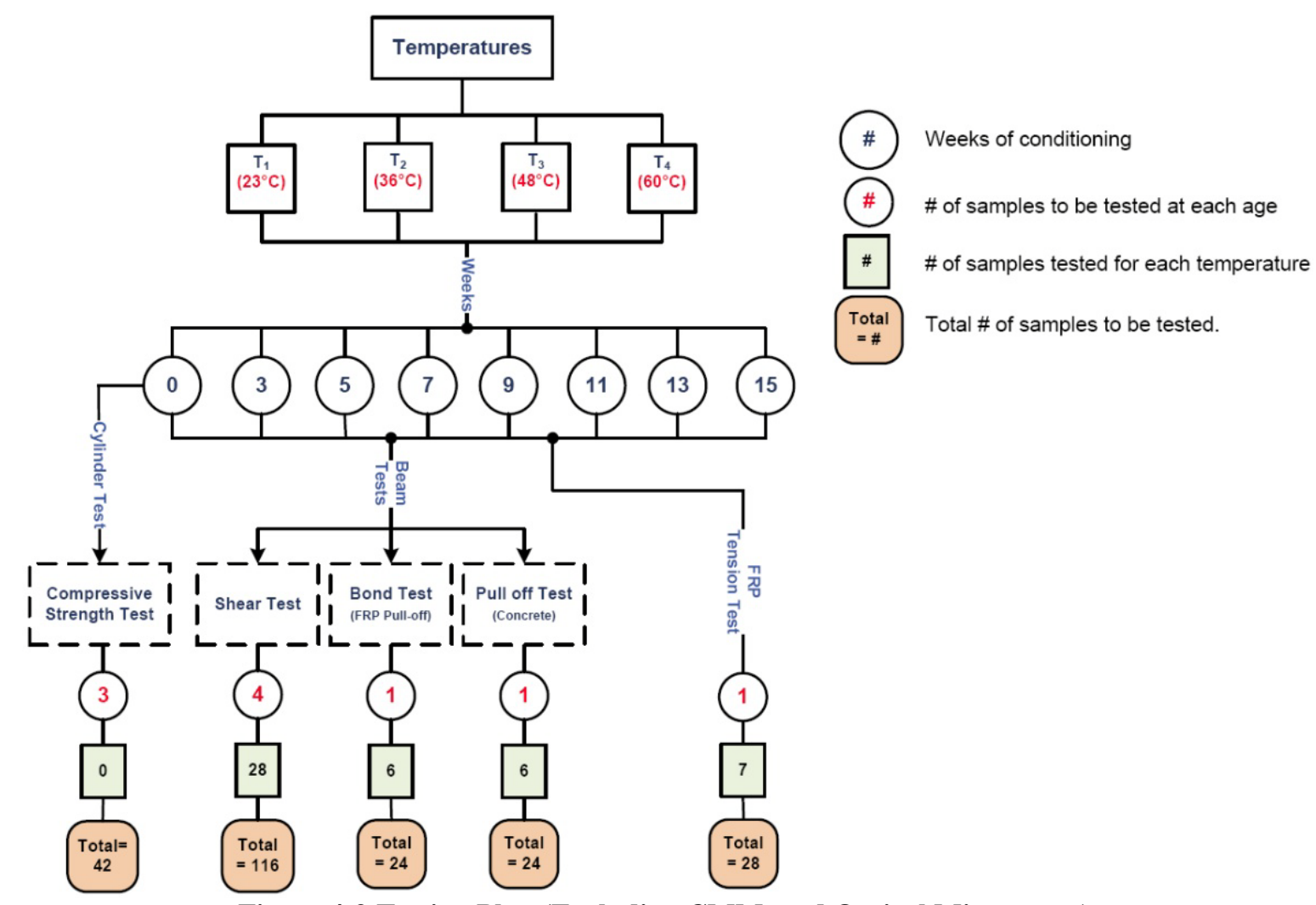

Figure 4-9 Testing Plan (Excluding CMM, and Optical Microscopy)

\subsubsection{Pull-Off Testing}

Pull-off testing was performed as per ASTM D 4541-02 on a set of CFRP-bonded beams to measure the both the bond strength off the FRP-to-concrete interface, and the concrete surface tensile strength. Dyna Z6 pull of tester from Proceq was used with aluminum dollies of 2 in (50 $\mathrm{mm}$ ) diameter (Figure 4-10).

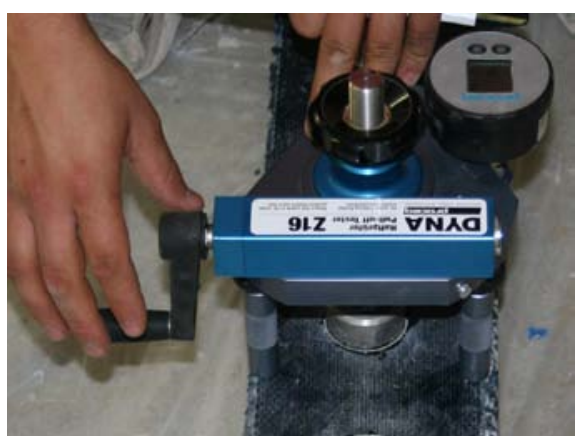

Figure 4-10 Pull off tester

First, the FRP surface was cleaned using pressurized air, and then core cutting was performed using a diamond core drill. The dollies' surfaces were slightly abraded, and then the 
epoxy ${ }^{1}$ was applied to both the dolly and the specified spot on the FRP. The dolly was pressed firmly against the FRP, and the excess epoxy was removed using a piece of shop towel. Some weights were left on top of the dollies and the epoxy was allowed to cure for 48 hours.

The pull-off tester was placed over the dolly and locked around the dolly's head. Then, the loading apparatus was pumped by hand at a slow constant rate until the failure. The load in which the FRP was pulled off the concrete and failure modes were recorded.

There are three possible failure modes in a pull-off test: (1) at the interface between the epoxy and the dolly (the test is inconclusive and the epoxy or the type of dolly should be changed and the assessment should be redone); (2) at the FRP-concrete interface (also known as adhesive failure); or (3) in the concrete substrate layer (also known as cohesive failure). The preferred mode of failure is in the concrete substrate layer, or simply the cohesive failure. If the failure occurs at the FRP and the concrete interface (adhesive failure), the bond is likely insufficient.

After the FRP pull-off test was complete, the side surface of the beam which was not FRPbonded was used to run the concrete pull-off, test in order to measure the concrete surface tensile strength. The same procedure which was used for the FRP pull-off was followed. There were only two modes of failure in this case, as there was no FRP engaged: (1) at the interface between the epoxy and the dolly; (2) in the concrete substrate layer (cohesive failure)

\subsubsection{Obtaining Surface Profile using Coordinate Measuring Machine (CMM)}

A 3-D surface profile was obtained using CMM. The machine was Checkmaster 216-142 from HELMEL Engineering (Figure 4-12). The software which recorded the coordinates was GEOMET 301 7.01.051. As the recording arm was not long enough to reach the FRP, the bench level was elevated using a box. In order to restrain the FRP movement while collecting the sample points’ coordinates a temporary frame was made using the adhesive (Figure 4-11).

After setting an arbitrary point as the zero of the machine coordinate system (MCS), the arm was locked against moving in the $\mathrm{X}$ direction (width of FRP). The coordinates of forty points were recorded along 6 different paths along the length of the FRP laminate making a total of 240 data points. After recording all the coordinates, the results were exported in a txt file.

\footnotetext{
${ }^{1}$ The saturant was used as the epoxy.
} 
Coordinates of the MCS were subtracted from the coordinates corresponding to the first data point.

Origin 8.1 professional version was used for data analysis and obtaining the fracture surface contour, and the fracture surface area. In order to calculate the surface area, Origin 8.1 uses a built-in "Renka-Cline" function. This function is especially advantageous in case of interpolation of small number of randomly spaced data. This method is more accurate for small data and is a triangle-based interpolation method. The following is a brief explanation of how it works:

- The XYZ coordinates are converted to a matrix.

- First triangulation is performed on XY data (nearly equal triangulation). It should be mentioned that near the boundaries there are more errors.

- Then gradient is estimated in the XY dimensions for each node as partial derivatives of a quadratic function.

- Then interpolation for an arbitrary point is done by computing the interpolated value using the data values and gradient estimate at each of the three vertices of the triangle containing the point.

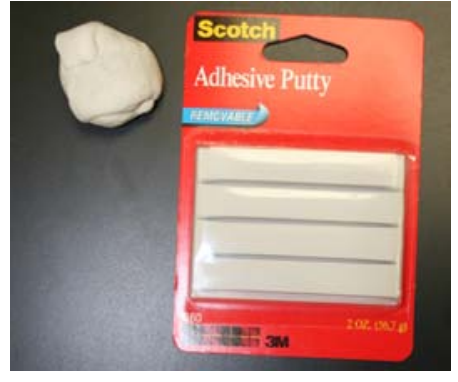

(a)

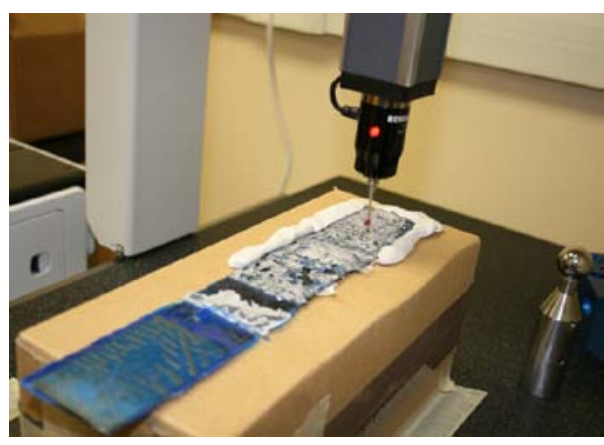

(b)

Figure 4-11 (a) Adhesive Putty, (b) Temporay Putty Frame 


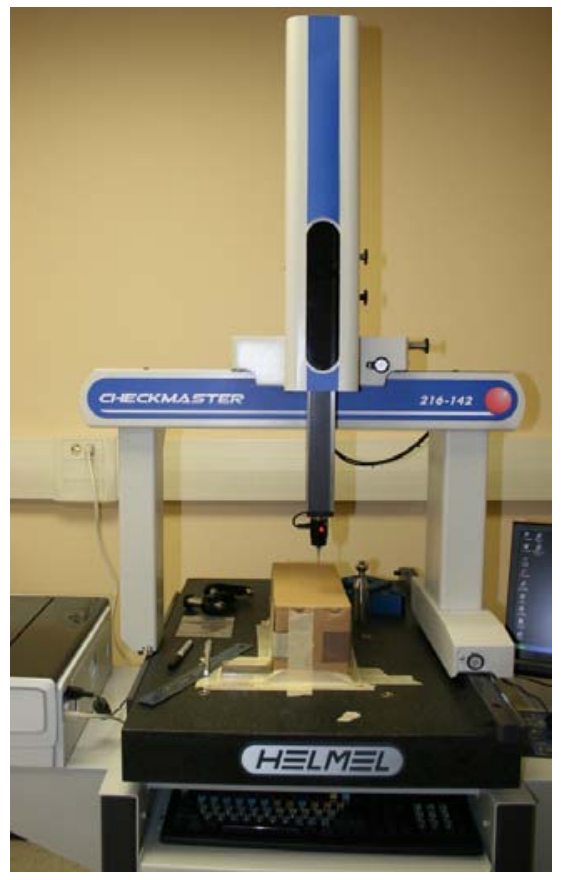

(a)

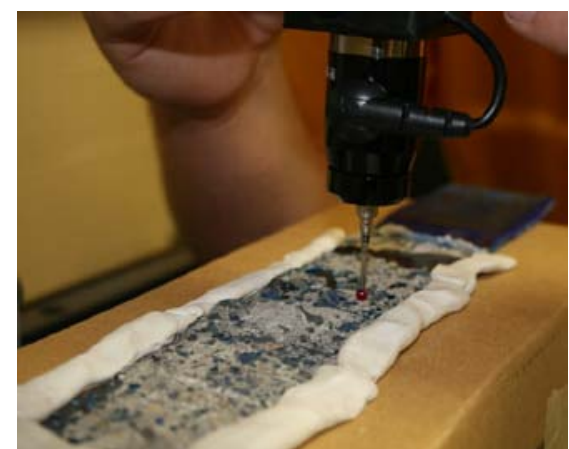

(b)

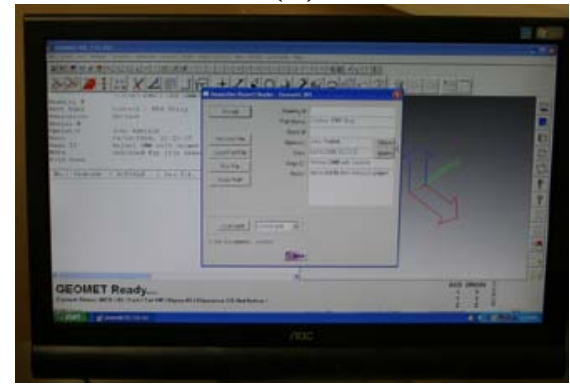

(c)

Figure 4-12 (a) CMM, (b) Recording the Coordinates, (c) GEOMET

\subsubsection{Interface Relative Humidity (IRH) Measurement}

Humitest system from James Instrument was used to measure the Interface Relative Humidity (IRH). The IRH of 4-5 points were measured per specimen. The measurements were done on companion specimens along with the shear tests.

After drilling the holes half an inch deep, the plastic sleeve was put in the hole and sealed with the plastic plug covered by the cap. It was left for 72 hours so that the interface humidity and the air entrapped in the cover reach stability. Then the IRH was recorded in percentage.

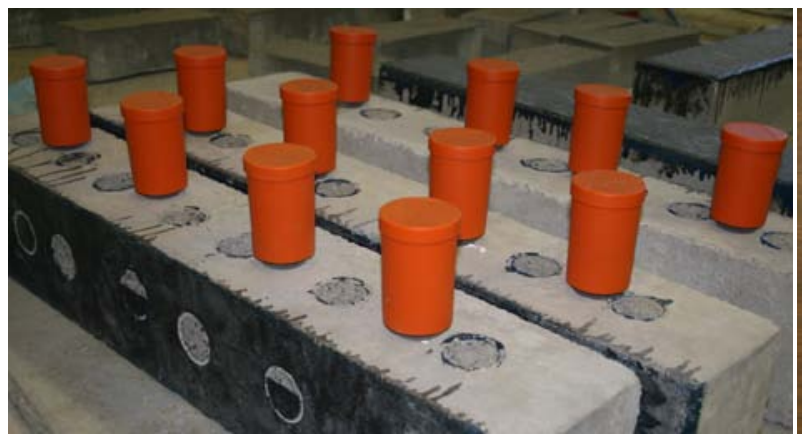

(a)

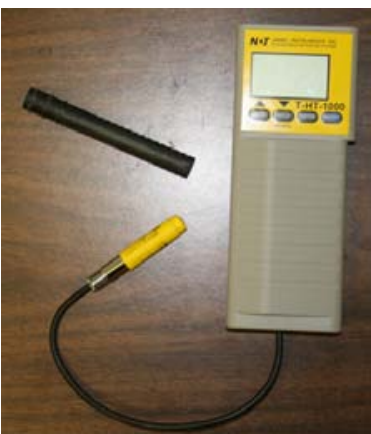

(b)

Figure 4-13 (a) Humidity measurement, (b) Humitest 


\subsubsection{Stereo Microscopy}

Olympus SZX12 stereo microscope was used to record optical images of the fracture surface on the delaminated CFRP (Figure 4-14).

14 representative delaminated CFRP's were selected to record the images. For this purpose, magnification of $\times 10$ was used.

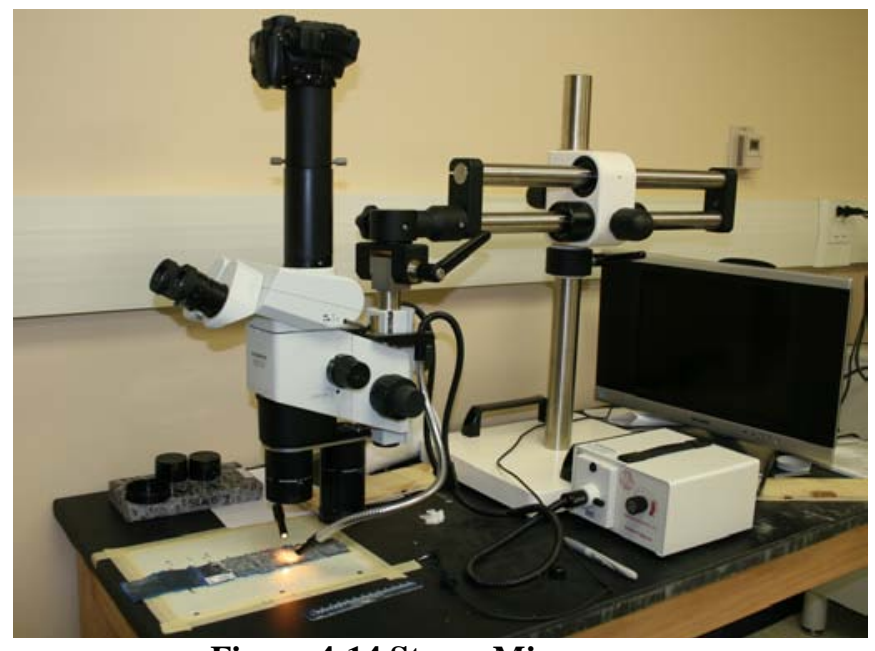

Figure 4-14 Stereo Microscope

The images were saved in jpeg format and then processed in Image-Pro Plus 6.0 image processing software. As it was not possible to record only one image from the entire $160 \mathrm{~mm}$ of the bond length, a total of 12 images were recorded for each laminate. Each laminate was divided into four equal zones and three images were recorded per zone (See Figure 4-15).

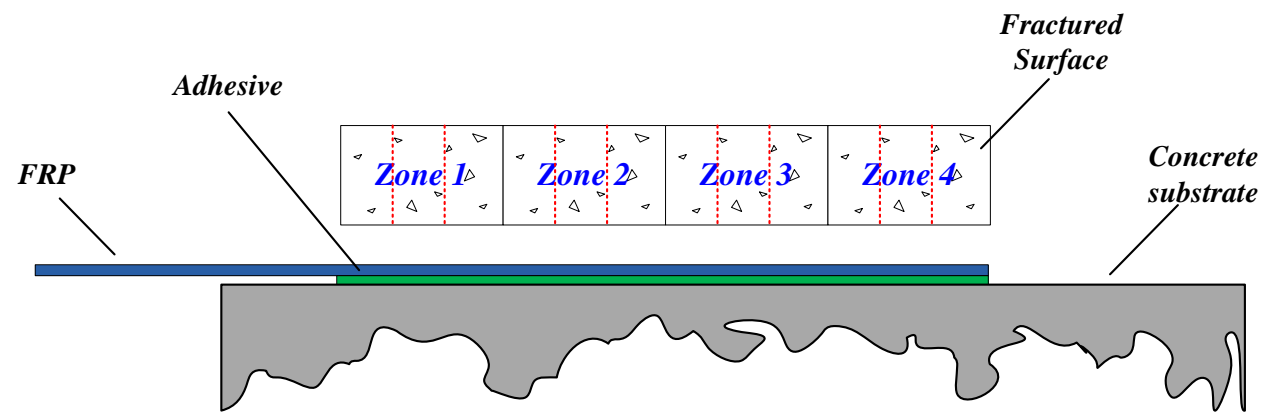

Figure 4-15 Fractured Surface Division for Recording Optical Images

Three color codes were identified in each image: blue, gray, and black, representing epoxy, concrete, and fibers, respectively. Using the trace tool of the image processing software, these areas were separated by drawing several polygons on the blue and the black area. In order to avoid any overlap, the gray area was calculated by subtracting the area of the blue and black area from the area of entire image. As these areas were selected based on the operator judgment and 
by eye-estimation only, it was important that the whole process would be done by only one individual to reduce the risk of different judgments and obtaining scatter results. After selecting the region of each color in a given image, the software calculated the corresponding area in terms of pixels. All these data were exported into an Excel file. The areas corresponding to each color were averaged out to obtain the percentage of each respective color on the delaminated CFRP.

It is worth mentioning that this technique does not provide any information regarding the thickness of the concrete attached to the CFRP.

It should be noted that in an ideal failure, no black area is expected. Simply because the existence of fibers on the fractured surface indicates that the fibers in that specific location were not impregnated completely; this is inevitable in hand lay-up method of FRP application. The other possibility for a reason behind formation of these black areas is that the epoxy did not have enough time to cure.

\subsubsection{FRP Tension Coupon Testing}

Direct tension tests were performed on two sets of different FRP specimens; the pristine FRP samples, and those delaminated from the concrete prism in single shear tests.

A total of 36 FRP specimens were tested as per ASTM D3039/D 3039M-00, 8 of which were pristine samples, and 28 of which were the delaminated laminates.

The pristine tension test specimens were made by first producing single ply 12 " $\times 12^{\prime \prime}$ in $\times$ in $(305 \times 305 \mathrm{~mm} \times \mathrm{mm})$ laminates. The laminates were kept in a humidity-controlled chamber at $77^{\circ} \mathrm{F}\left(25^{\circ} \mathrm{C}\right)$ and $50 \% \mathrm{RH}$ for 7 days for the FRP to be cured. Upon curing, the laminates were cut into coupons with 12" length and 1" width. Four of these coupons were tested with grips and four without grips. $3^{\prime \prime} \times 1$ " in $\times$ in $(76 \times 25 \mathrm{~mm} \times \mathrm{mm})$ aluminum grips were made out of $1 / 8^{\prime \prime}$ sheets. The grips were first lightly sanded on the side to be bonded to the FRP, and then epoxy was used to bond the grips to each coupon. The same saturant was used as the epoxy for this purpose. After curing, the coupons were tested at a constant rate of $2 \mathrm{~mm} / \mathrm{min}$ up to failure using the same MTS machine used for the single shear tests.

As for the delaminated specimens, one representative sample of each group of specimens corresponding to a specific temperature and exposure time was tested, i.e., a total of 28 
delaminated samples were tested. These laminates were tested as they were, and no grip was bonded. If any concrete was attached to the laminate, it was left as it is.

\subsection{Data Reduction Methods}

The traction-separation law in this study was obtained using two methods: (1) the J-integral method based on fracture mechanics; and (2) the traditional method based on the FRP strain distribution captured using strain gages mounted on the FRP layer. Section 4.7.1 explains the new J-integral method as a reliable method to obtain the constitutive law for FRP-concrete interface measuring only the load and slip at the crack tip. The traction-separation law is then obtained using the traditional strain-based approach in section 4.7.3. The fracture energy is obtained as the area under each curve, and the results are compared against each other, and the well accepted equation (2-20) in the literature.

\subsection{Traction Separation Law}

The following assumptions are adopted for data analyses:

- Both concrete and FRP to be elastic materials.

- Thickness variation of FRP laminate is neglected.

- Normal stress at the interface is neglected (assuming that the interface is subjected only to shear stress).

\subsubsection{J-integral Method}

In this method the interfacial fracture energy and the traction-separation law are obtained by solely measuring the load and the slip at the tip of the predefined notch. By avoiding the cumbersome, time consuming procedure of mounting strain gages, a considerable amount of time and energy can be saved and thus, this method can be a proper substitute for the traditional strain-based method.

\subsubsection{Load-Slip Curve Fitting}

To begin, the load is approximated as an exponential function of slip in the following general form:

$$
N=a\left(e^{b \delta}-1\right)
$$


where $N$ is the load, $\delta$ is the slip measured by a spring Loaded LVDT at the tip of the predefined notch, and $a$ and $b$ are empirical constants obtained by Newton-Raphson regression method. This curve removes the load variations which are caused by the materials inhomogeneity. Figure 4-16 depicts a typical load-slip curve using Equation (4-1). As illustrated in Figure 4-16, three distinct regions can be identified in this curve. In region (i) as the relative slip progresses, the load-displacement curve is increased linearly up to point A. The load increases linearly afterwards in region (ii) up to point B. After this point, the load remains almost constant in region (iii) up to failure.

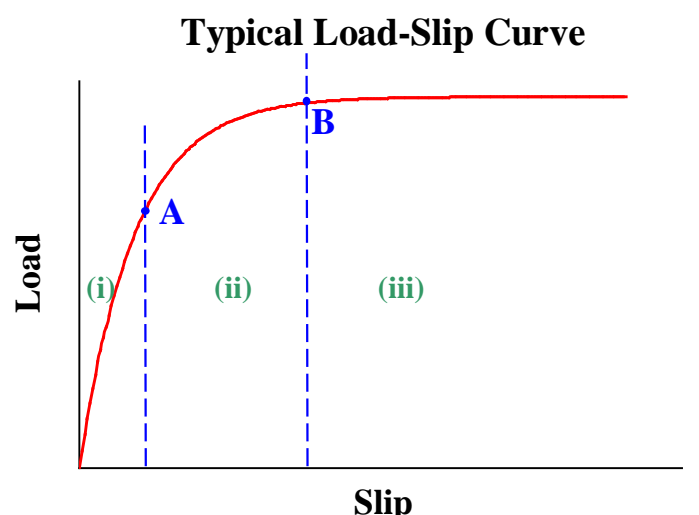

Figure 4-16 Typical Load-Slip Curve

\subsubsection{J-Integral Approach}

Rice (1968) defined fracture energy as a line integral along an arbitrary path around the crack or the notch. This integral, which is path-independent, is called J-integral. The appropriate defining path for J-integral would be that which includes the crack tip. For linear/nonlinear elastic materials under uniaxial loading or elastic-plastic ones subjected to deformation only, defined the two dimensional J-integral as follows:

$$
\begin{aligned}
& J=\oint_{\Gamma}\left(w d z-\vec{T} \cdot \frac{\partial \vec{u}}{\partial x} d s\right) \\
& w=w_{(\varepsilon)}=\int_{0}^{\varepsilon} \sigma_{i j} d \varepsilon_{i j}
\end{aligned}
$$

where $\vec{u}_{\text {is }}$ the displacement vector; $\vec{T}$ is the traction vector acting outward normal to $\Gamma$ defined as $T_{i}=\sigma_{i j} n_{j} ; \sigma_{i j}$ and $\varepsilon_{i j}$ are the components of stress and strain tensors; ds is an element of infinitesimal length along $\Gamma$; $\Gamma$ is a closed path surrounding the notch; and $w$ is the strain energy defined as: 
Keeping this in mind, the close path $\Gamma$ is taken as the integration path for the specimen in this study (Figure 4-17).

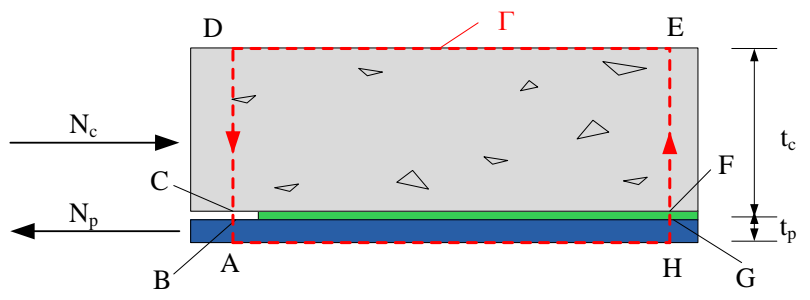

Figure 4-17 J-integral path

For the path shown in figure above, Equation (2-8) becomes:

$J=J_{A H}+J_{H G}+J_{G F}+J_{F E}+J_{E D}+J_{D C}+J_{C B}+J_{B A}$

However, among all the terms in Equation (4-4), only sections BA and DC contribute to the net $\mathrm{J}$ integral as the traction force on all the other sections is zero making $J_{i j}$ for the other sections zero. Thus Equation (4-4) reduces to:

$J=J_{D C}+J_{B A}$

where:

$$
\left\{\begin{array}{l}
J_{D C}=\int_{0}^{t_{c}}\left(w d z-\vec{T} \cdot \frac{\partial \vec{u}}{\partial x} \cdot d s\right) \\
J_{B A}=\int_{0}^{t_{p}}\left(w d z-\vec{T} \cdot \frac{\partial \vec{u}}{\partial x} \cdot d s\right)
\end{array}\right.
$$

Since the traction forces and $d s$ in sections DC and BA are perpendicular, the second term of Equations (4-6) -a, and (4-6) -b becomes zero.

Substituting $\sigma_{i j}=E \varepsilon_{i j}$ in Equation (2-9), the strain energy for each of the two sections is obtained as follows:

$$
\begin{aligned}
w=w_{(\varepsilon)} & =\int_{0}^{\varepsilon} \sigma_{i j} d \varepsilon_{i j}=\int_{0}^{\varepsilon} E \varepsilon_{i j} d \varepsilon_{i j} \\
& =E \varepsilon_{i j}{ }^{2}=\frac{E}{2}\left(\frac{\sigma_{i j}}{E}\right)^{2} \\
w & =\frac{1}{2} \frac{\sigma_{i j}{ }^{2}}{E}
\end{aligned}
$$


Substituting Equation (4-7) into Equation (4-6), and using $\sigma_{k}=N_{k} / A_{k}$ (where $k=c$ for concrete or $p$ FRP):

$$
\left\{\begin{array}{l}
J_{D C}=\int_{0}^{t_{c}} w d z=1 / 2 \int_{0}^{t_{c}} \frac{\sigma_{c}^{2}}{E}=1 / 2\left(\frac{N_{c}{ }^{2}}{A_{c}{ }^{2} E_{c}} \times t_{c}\right) \\
J_{B A}=\int_{0}^{t_{p}} w d z=1 / 2 \int_{0}^{t_{c}} \frac{\sigma_{p}{ }^{2}}{E}=1 / 2\left(\frac{N_{p}{ }^{2}}{A_{p}{ }^{2} E_{p}} \times t_{p}\right)
\end{array}\right.
$$

Therefore from Equations (4-5) and (4-8), the J-integral reduces to:

$$
J=\frac{1}{2} \frac{N_{p}^{2}}{A_{p}^{2} E_{p}} \times t_{p}+\frac{1}{2} \frac{N_{c}^{2}}{A_{c}^{2} E_{c}} \times t_{c}
$$

Since the axial stiffness of FRP laminate is much smaller than concrete, i.e., $E_{p} A_{p}<<E_{c} A_{c}$, the concrete terms in Equation (4-9) can be neglected. Therefore, the simplified J-integral is:

$$
J=\frac{1}{2} \frac{N_{p}^{2}}{A_{p}^{2} E_{p}} \times t_{p}
$$

Since $\mathrm{J}$ is equal to the Energy Release Rate (ERR) at the debonding tip, the shear stress at the interface can be obtained using:

$$
\tau=\frac{d J}{d \delta}
$$

Substituting Equation (4-10) into Equation (4-11), the following relation between the interfacial shear stress and the slip at the tip of the notch can be obtained:

$$
\tau=\frac{1}{2 A^{2} E t} \frac{d}{d \delta}\left(N^{2}\right)
$$

This equation is used in the data analysis to obtain the traction-separation law of the interface, where $N$ is the approximated load from Equation (2-1), and $\delta$ is the corresponding slip recorded by the LVDT. Substituting Equation (4-1) into Equation (4-12), shear stress is obtained as a function of slip:

$$
\tau=\frac{A_{p}^{2} B e^{B s}\left(e^{B S}-1\right)}{a_{p} b_{p} E_{p}}
$$

When $N$ is equal to the maximum load, $\mathrm{J}$ becomes fracture energy release rate, $G_{f}$. Using Equation (4-10), ERR is as follows: 
$G_{f}=\frac{1}{2} \frac{N_{\max }^{2}}{A_{p}^{2} E_{p}} \times t_{p}$

which is essentially the same as the following equation, which was derived by researchers Taljsten (1996); Yuan et al. (2001); Wu et al. (2002), assuming an infinite bond length, using LEFM approach (See Equation (2-20)):

$$
F_{\max }=b_{P} \sqrt{2 E_{P} t_{P} G_{f}}
$$

Therefore, ERR of the interface can be found by obtaining the maximum applied load only, and is defined as the integration of the Equation (4-12) with respect to the slip, or simply the area under the shear stress-slip curve. Thus, Equation (4-14) can be used to verify the obtained traction-separation law by comparing the area under the $\tau$ - $\delta$ curve and Equation (4-14). Based on the assumption regarding bond length which was made in order to derive Equations (4-12) and (4-14), that bond length tends to infinity, Equation (4-14) can be used only for experiments in which the bond length is sufficiently long (longer than the effective length).

The traditional method of obtaining the interface constitutive law is discussed in the next section.

\subsubsection{Traditional Method Using Mounted Strain Gages}

The traditional method was developed by Taljsten (1997), and has been used by many other researchers ever since to obtain the interfacial constitutive law (Pham and Al-Mahaidi, 2006; Nakaba et al., 2001; and Ferracuti, 2006).

Here, the strain distribution along the FRP laminate is obtained using strain gages mounted on top of the laminate. In order to obtain the relative slip between FRP and concrete at each point along the FRP, it is assumed that strain gage located farthest from the loaded end is perfectly bonded to the FRP. That is the slip at that point is zero. In other words, $s\left(x_{n}\right)=0$. Moreover, as the axial stiffness of FRP laminate is much smaller than that of the concrete $\left(E_{P} A_{P}<<E_{C} A_{C}\right)$, it can be assumed that the concrete slip is negligible compared to that of FRP, making the relative slip between the two sections only related to FRP. It is also assumed that strain varies linearly between each of the two successive strain gages as shown in Figure 4-18):

$$
\varepsilon(x)=\frac{\varepsilon_{i+1}-\varepsilon_{i}}{x_{i+1}-x_{i}}\left(x-x_{i}\right)+\varepsilon_{i}
$$


Thus, the relative slip between concrete and FRP at the place of a gage located at a distance $x$ from the loaded end is obtained by integrating the above equation over the distance $x$ :

$$
\begin{aligned}
& s(x)=\int_{x_{0}}^{x} \varepsilon(x) d x \\
& s(x)=s\left(x_{i+1}\right)+\frac{1}{2} \frac{\varepsilon_{i+1}-\varepsilon_{i}}{x_{i+1}-x_{i}}\left(x-x_{i}\right)^{2}+\varepsilon_{i}\left(x-x_{i}\right) ; \quad x_{i} \leq x \leq X_{i+1}
\end{aligned}
$$

Assuming that the FRP laminate is perfectly bonded to the concrete (i.e., the slip at the strain gage which is farthest from the loaded end is zero, $s\left(X_{n}\right)=0$ ) the slip at each strain gage location can be calculated. The average slip in the middle of two successive strain gages, $s_{i}$, can be expressed as:

$$
s_{i}=\frac{s\left(x_{i+1}\right)+s\left(x_{i}\right)}{2}
$$

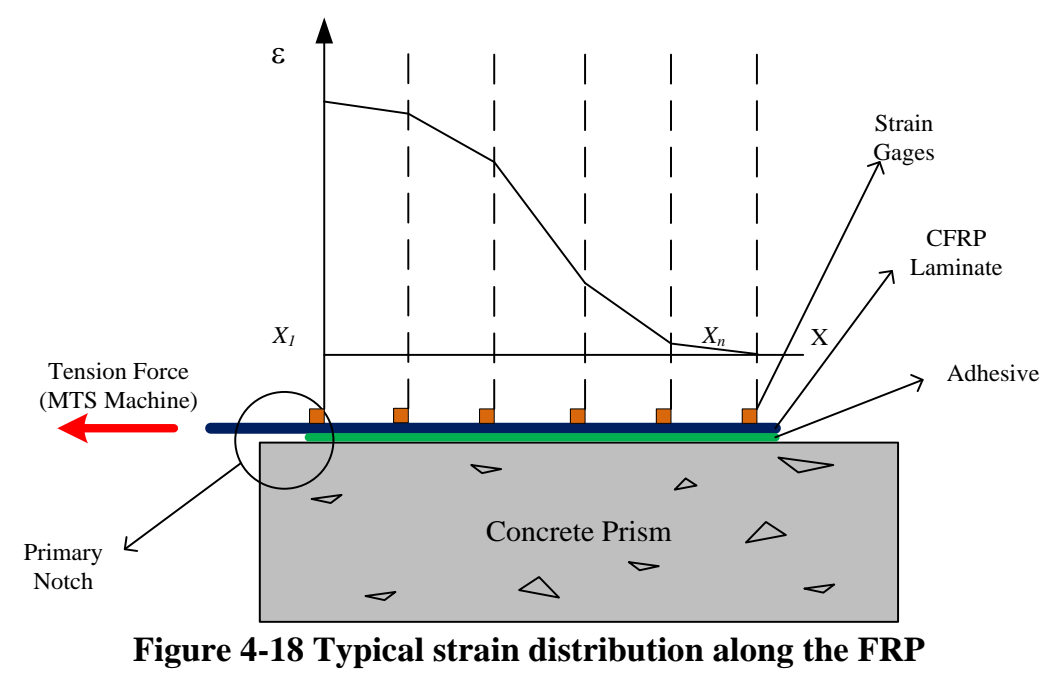

The shear stress can be obtained considering the equilibrium of an infinitesimal element of FRP as shown in Figure 4-19.

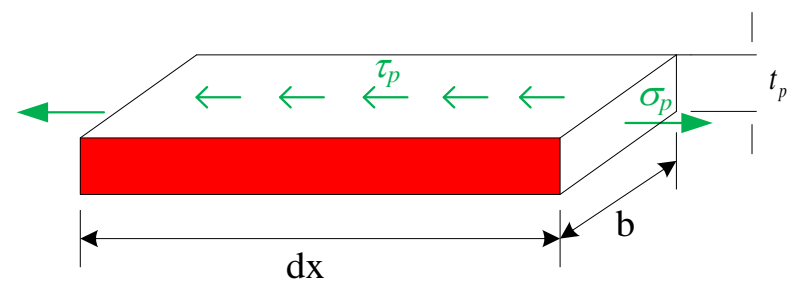


Figure 4-19 Equilibrium of an infinitesimal FRP element

$\tau(b d x)=[(\sigma+d \sigma)-\sigma] t b \quad \rightarrow \quad \tau(d x)=t(d \sigma)$

Substituting $d \sigma=E d \varepsilon$ in the above equation gives the shear stress, $\tau_{i}$, in the middle of each two successive strain gages as a function of strain gradient:

$\tau_{i}=t_{P} E_{P} \frac{d \varepsilon(x)}{d x}=t_{P} E_{P} \frac{\varepsilon_{i+1}-\varepsilon_{i}}{x_{i+1}-x_{i}}$

where $t_{p}$ and $E_{p}$ are the FRP thickness and its modulus of elasticity, respectively. It is assumed that the shear stress between each two successive strain gages is constant (See Figure 4-20).

Finally, the traction-separation law obtained by the traditional method can be plotted as $\tau_{i}$ versus $s_{i}$.
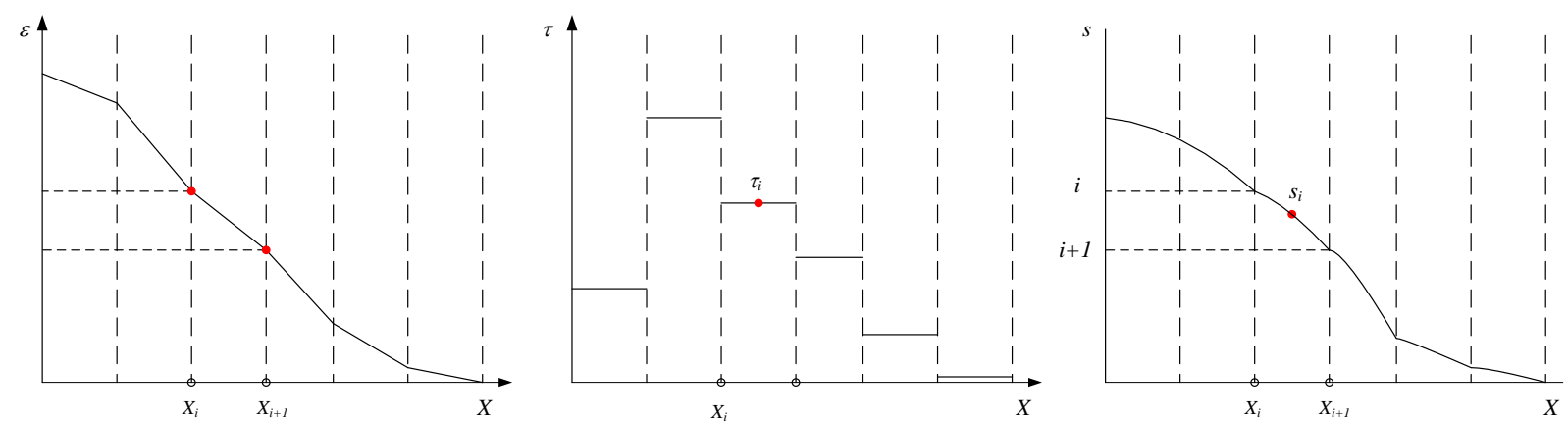

Figure 4-20 (a) Strain Distribution as obtained from Strain gages, (b) average shear stress, (c) average slip between FRP and concrete

As mentioned earlier, the traditional approach has been used by many other researchers throughout the years, namely Nakaba et al. (2001), Ferracuti (2006), and Pham and Al-Mahaidi (2007), who used more or less the same method of numerical integration in order to find the relative slip and the interfacial shear stress. Nakaba et al. (2001) took the deformation of the substrate into account and calculated it from an equivalent section. However, Ferracuti (2006) and many other researchers have neglected the concrete substrate deformation, compared to its FRP counterpart. 


\subsection{Fracture Energy}

Evaluation of the fracture energy of the interface is of great importance in order to modify the design codes because if the fracture energy is know in advance, the maximum transferrable load of the interface can be calculated using Equation (4-14). Calculation of the fracture energy by both J-integral, and traditional approaches are explained next.

\subsubsection{Fracture Energy: J-Integral Approach}

As mentioned earlier, the fracture energy is equal to the area under the shear stress-slip curve. In other words, the integral the shear stress function over the slip:

$$
G=\int \tau(x) d s(x)
$$

where $\tau(x)$ is substituted from Equation (4-12).

\subsubsection{Fracture Energy: Traditional Approach}

The fracture energy of the traditional strain-based method is obtained by numerical integration using a short code written in Maple.

\subsubsection{Fracture Energy: Literature}

The fracture energies obtained from the two above mentioned methods are compared to the energy calculated from Equation (2-20), which is a well-accepted equation in the literature to calculate the fracture energies of FRP-concrete bonds of adequate length.

\subsection{Summary}

In this chapter, the analytical basis of this study was discussed. The traction separation law and the interfacial fracture energy are obtained by two different methods: the J-integral approach, and the traditional approach. The J-integral method requires measuring only load and slip at the debonding end, thus saving considerable time and energy by omitting an otherwise required process of bonding strain gages for traditional methods to obtain the constitutive law for the interface. It also avoids the inherent assumption of linear elastic fracture mechanics (LEFM) behavior, as it is valid only when the size of deformation zone is small compared to the crack size, which is not the case for concrete or even some composite materials. 


\section{Testing Results and Discussion}




\subsection{Introduction}

The general failure mode observed throughout the experiment is explained first. Next, the results of numerous testing explained in chapter 4 including Mode II shear testing, FRP coupon testing, optical microscopy, and measuring water ions are presented and discussed in this chapter. First, in 5.6, the failure mode is explained qualitatively. Then the traction-separation laws ( $\tau$-s curves) obtained by both traditional strain-based method, and J-integral approach are presented in 5.3. The results are compared and discussed. The cohesive/adhesive failure percentiles were determined by processing these images. Then, in 5.6, images recorded by optical microscopy are presented. The surface profile contours as obtained using CMM are presented in 5.8 .

\subsection{Energy Release Rate}

ERR for all the specimens were calculated using Equation (4-14) which is recited here:

$$
G_{f}=\frac{1}{2} \frac{N_{\max }^{2}}{A_{p}^{2} E_{p}} \times t_{p}
$$

Since the maximum load is used in the above equation, the resulting $G_{f}$ would be the ERR. Four specimens were tested for each temperature at a given week. ERR for each specimen was calculated independently. If the coefficient of variance (COV) of the calculated ERR for a specimen was more than $20 \%$, the corresponding specimen was disregarded in data analysis. The

average ERR after omitting the outliers is plotted in Figure 5-1 for varying temperatures and exposure times: 


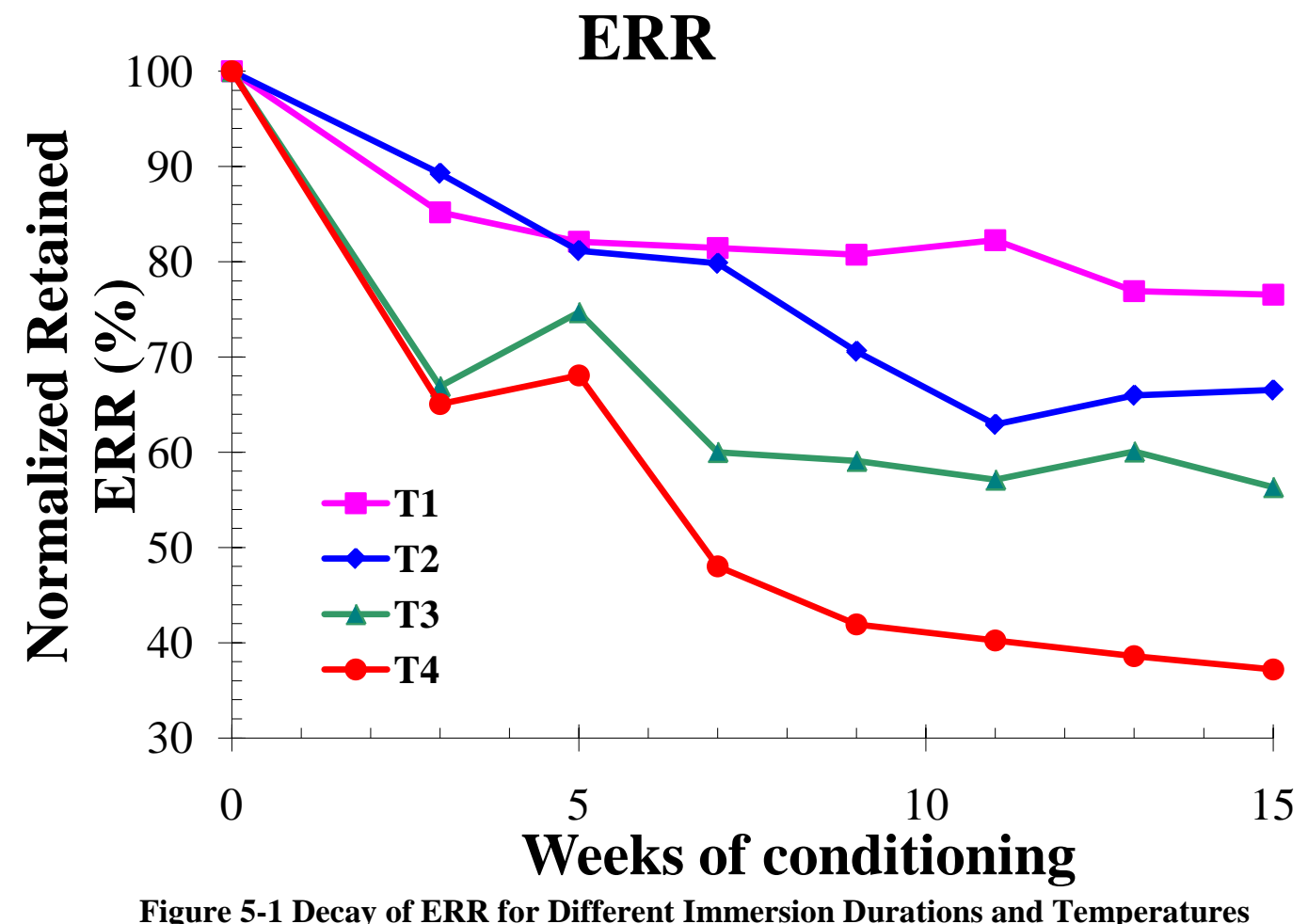

After three weeks of continuous water immersion, the ERR dropped slightly more than $10 \%$ for T1 and T2 samples. However, the ERR dropped more than 35\% for T3 and T4 samples for the same exposure time. After the $5^{\text {th }}$ week of water immersion, ERR for the T1 samples remained relatively constant until week 15 . This observation shows that temperature elevation has a more pronounced effect on interface deterioration than water immersion period.

Figure 5-2 illustrates ERR drop for a given week for different temperatures. With a few noted exceptions, general trend revealed a decrease of ERR when temperatures, and water immersion duration increase. 


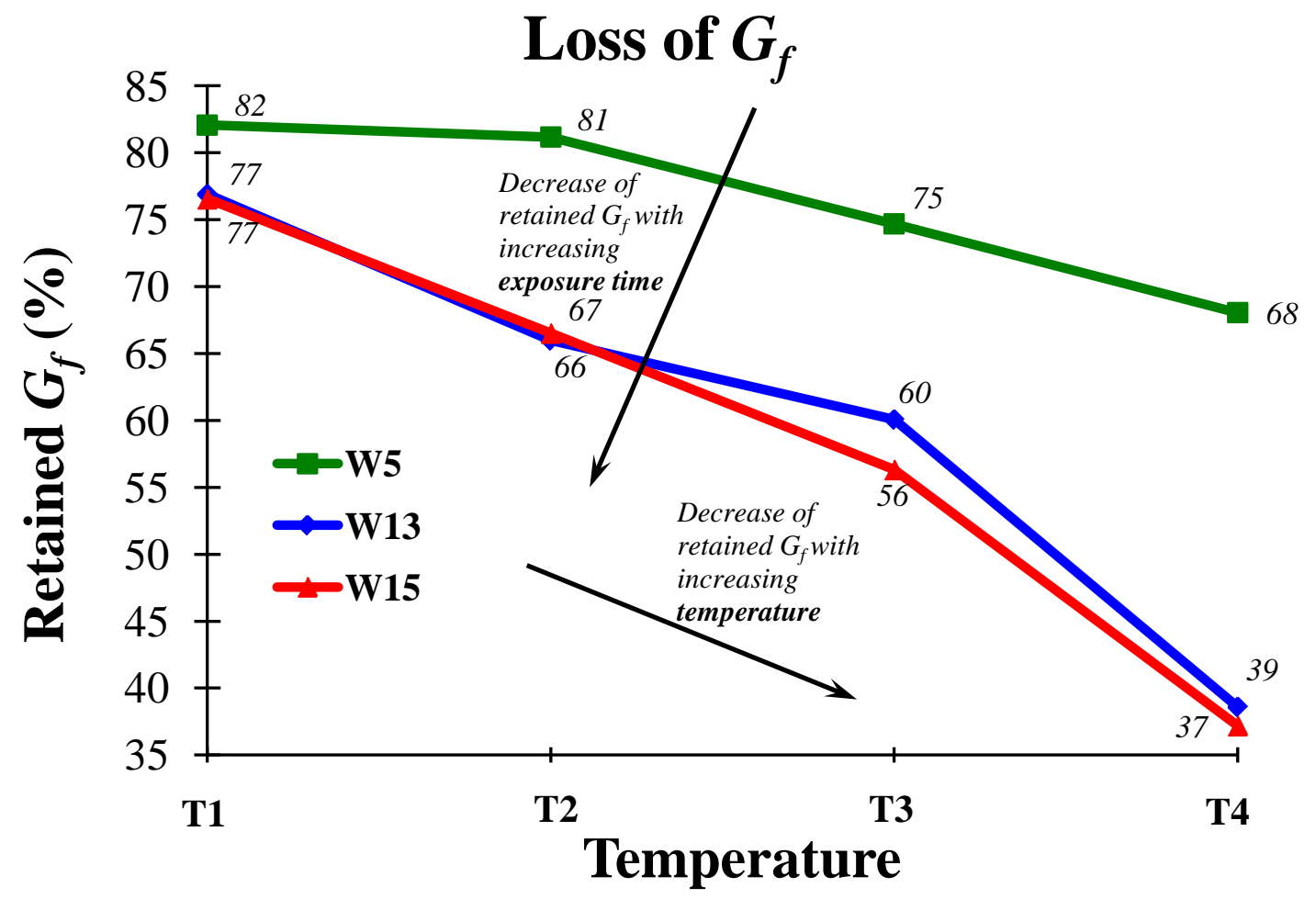

Figure 5-2 Decay of ERR for a Given Immersion Period (5, 13, and 15 weeks)

\subsection{Traction Separation Law}

Shear stress-slip curves, for different temperatures and exposure periods, obtained from both traditional and J-integral approaches are presented in this section. Due to misplacing the LVDT in the early stage of the experiment, the slip recorded for almost half of the specimens was disregarded, which prohibits application of the J-integral method for those samples. J-integral method is implemented to analyze the experimental data from the rest of the specimens with reasonable LVDT readings. However, the traditional method is applied to the entire specimens.

\subsection{J-integral approach}

As discussed in section 4.7.1, least square minimization is used to estimate the load as an exponential function of slip in the following form:

$$
N=A\left(e^{B \delta}-1\right)
$$

where $A$ and $B$ are empirical constants, $N$ is the regression results for the load and $\delta$ is the slip recorded by the LVDT during the experiment. Figure 5-3 illustrates a schematic view of a typical load-slip curve. As expected, as the relative slip progressed, the load-displacement path 
was increased linearly up to point $\mathrm{A}$, and then the load increased nonlinearly between points $\mathrm{A}$ and B. After this point, the load remained almost constant at until complete failure occurred.

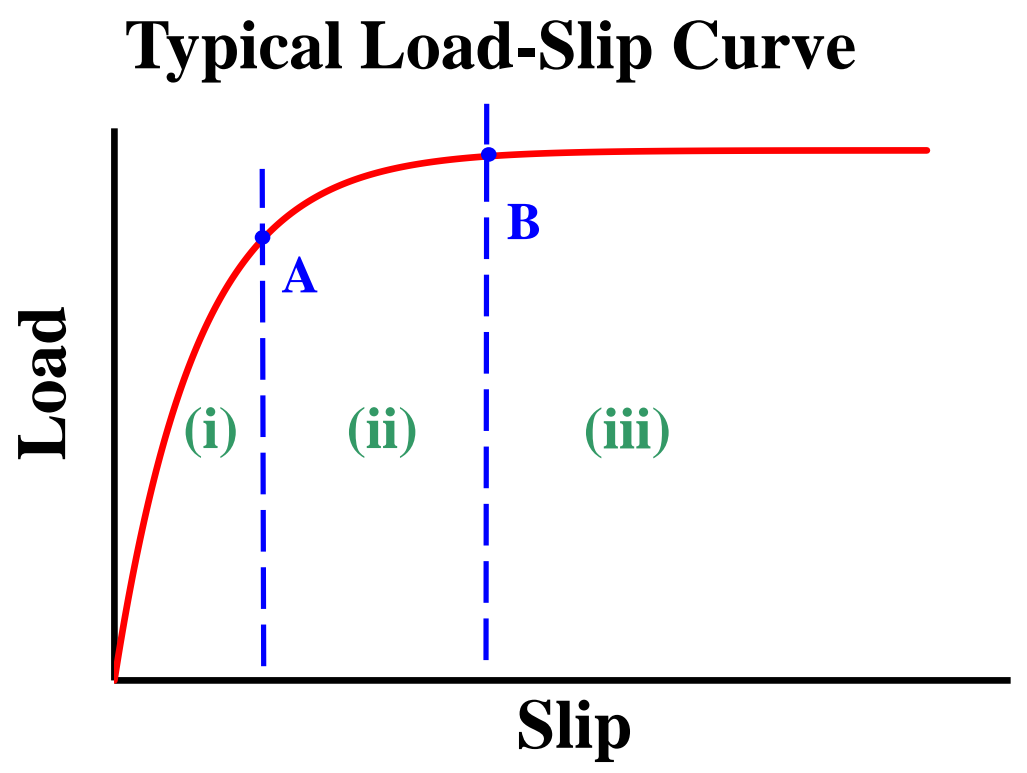

Figure 5-3 Schematic View of a Typical Load-Slip Curve

The following graphs illustrate the regression results for load versus slip. As can be seen from Figure 5-4, the general trend is decreasing the maximum load with increasing the temperature for the same exposure time. For week 11 and week 13, however, T2 and T3 slightly violate this general trend, i.e., the maximum load after 13 weeks of conditioning is higher than that after 11 weeks. 


\section{Regression Result vs. Slip (Week 11)}

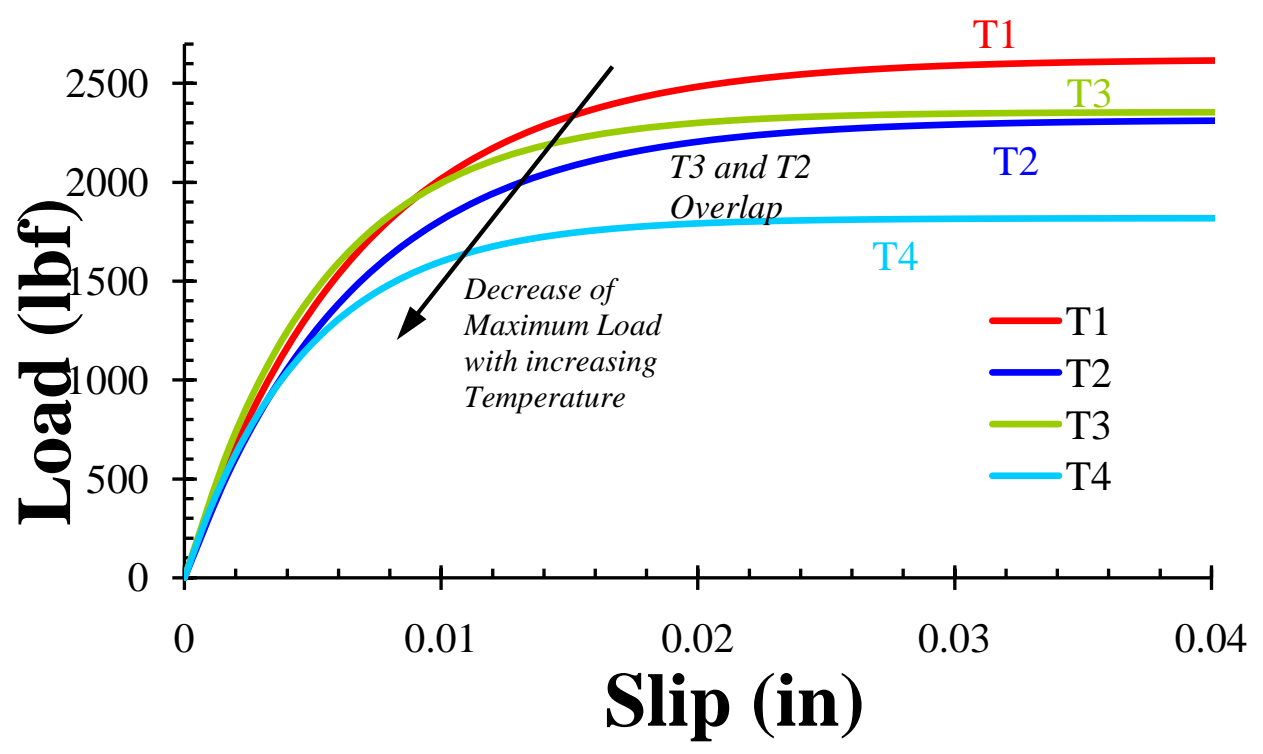

Figure 5-4 Predicted Load for Various Temperatures at Week 11

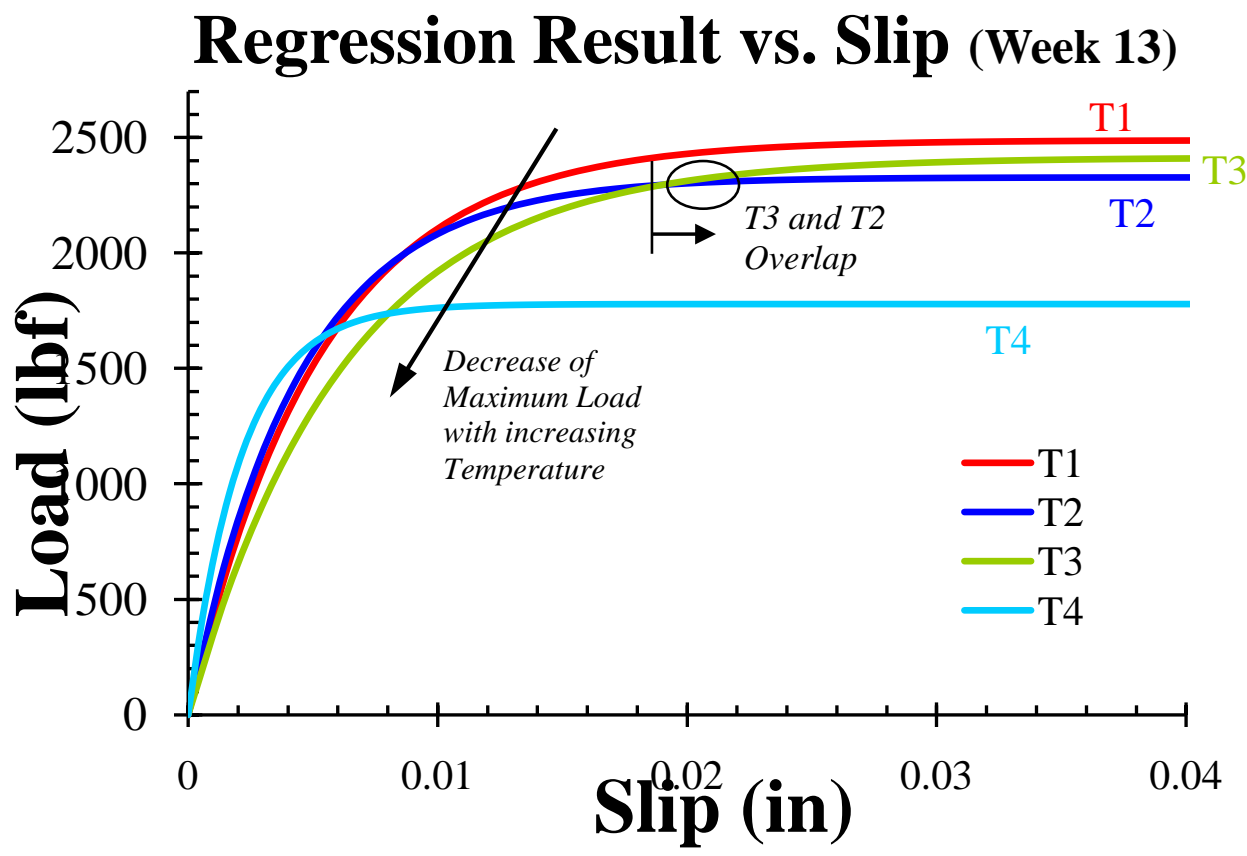

Figure 5-5 Predicted Load for Various Temperatures at Week 13 


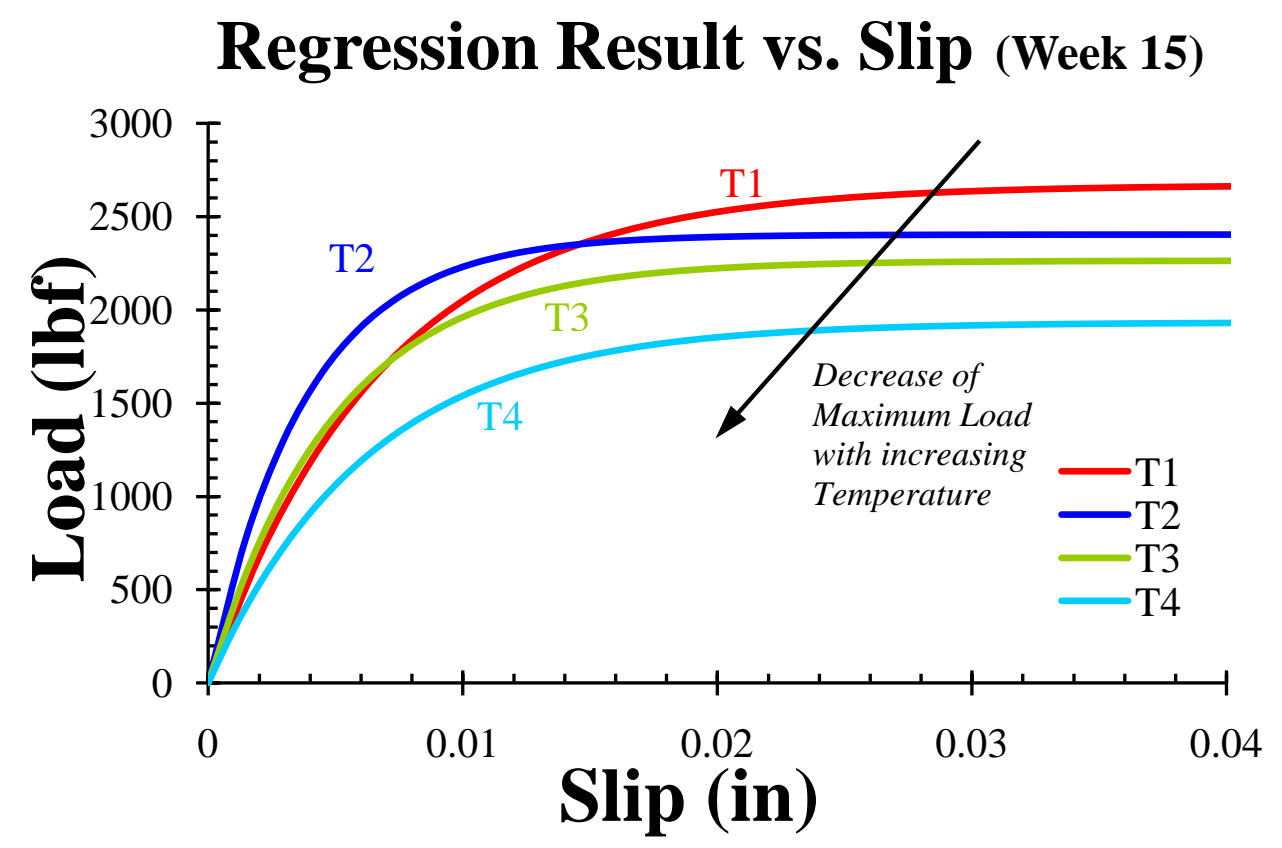

Figure 5-6 Predicted Load for Various Temperatures at Week 15

Figure 5-7 illustrates a representative experimental and Regression results versus slip curve. (Not are all the data points shown in the figure).

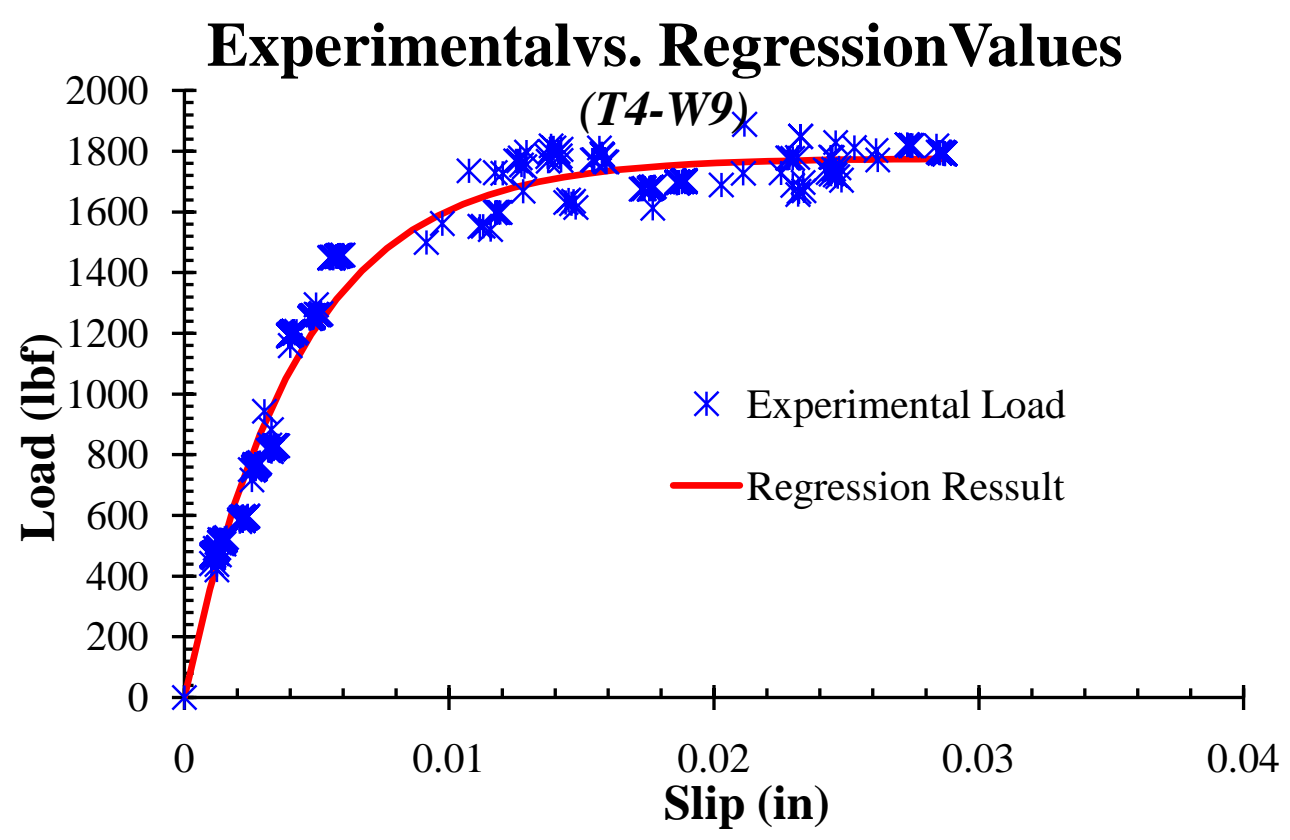

Figure 5-7 Experimental vs. Predicted Load Values (T4-W9)

Figure 5-8 shows the traction-separation laws obtained for three different weeks of immersion in T4 tanks using Equation (4-13). 


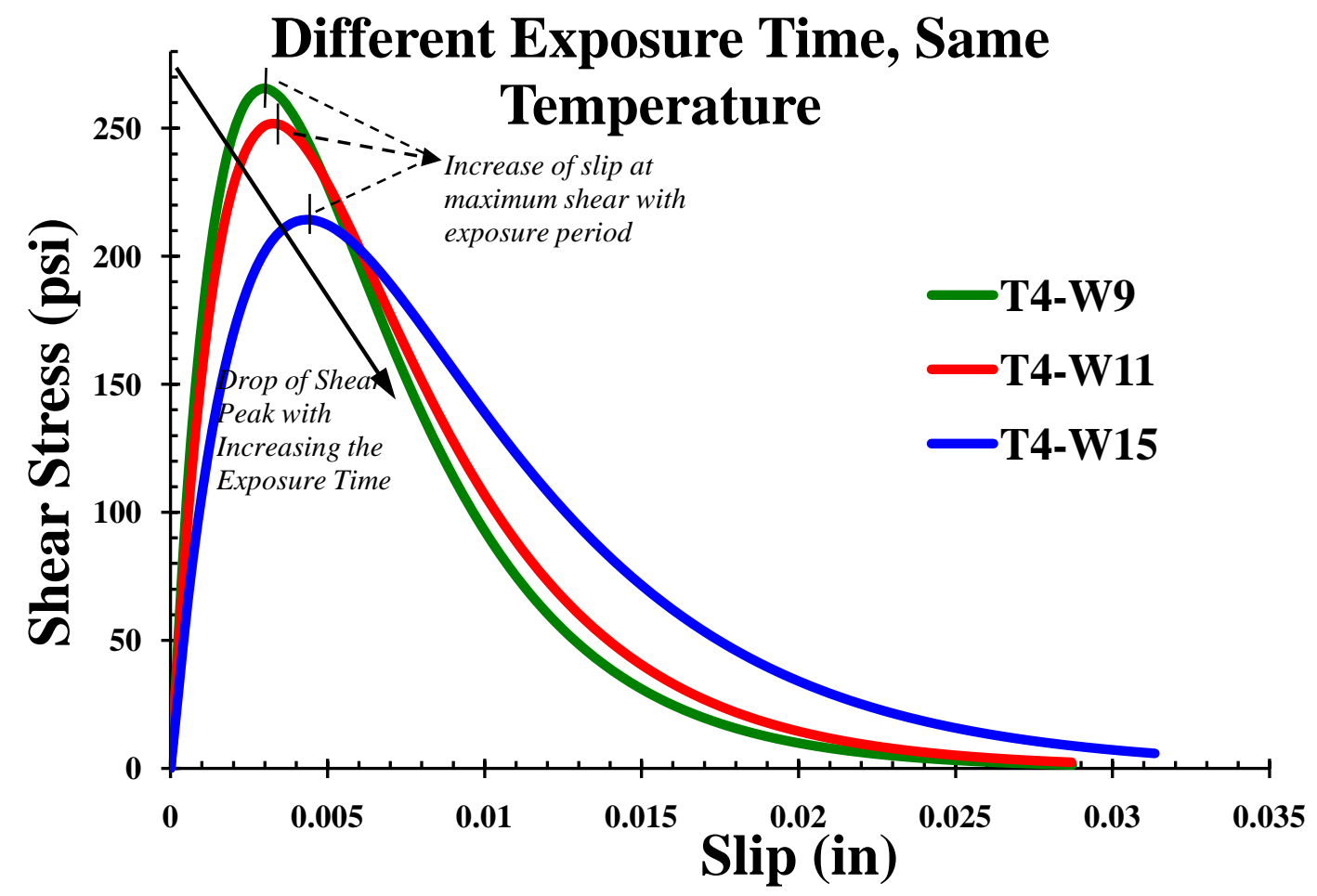

Figure 5-8 Traction-Separation Law

The relationship for each of the above curves can be obtained by substituting the empirical constants, A and B, into Equation (4-12).

\begin{tabular}{l|l|l|l}
\hline T4 & \multicolumn{3}{|l}{} \\
\hline & W9 & W11 & W15 \\
A & -1777.15 & -1818.85 & -1934.56 \\
$\boldsymbol{B}$ & -233.76 & -211.59 & -159.21 \\
$\boldsymbol{S}_{\max }$ & 0.028714 & 0.0287 & 0.03135 \\
\hline
\end{tabular}

As the exposure period increases, the maximum shear stress decreases, while the corresponding slip increases. However, not all the curves for various temperatures and exposure periods comply with this general trend. Traction-Separation laws obtained by each method are presented in the figures below. 


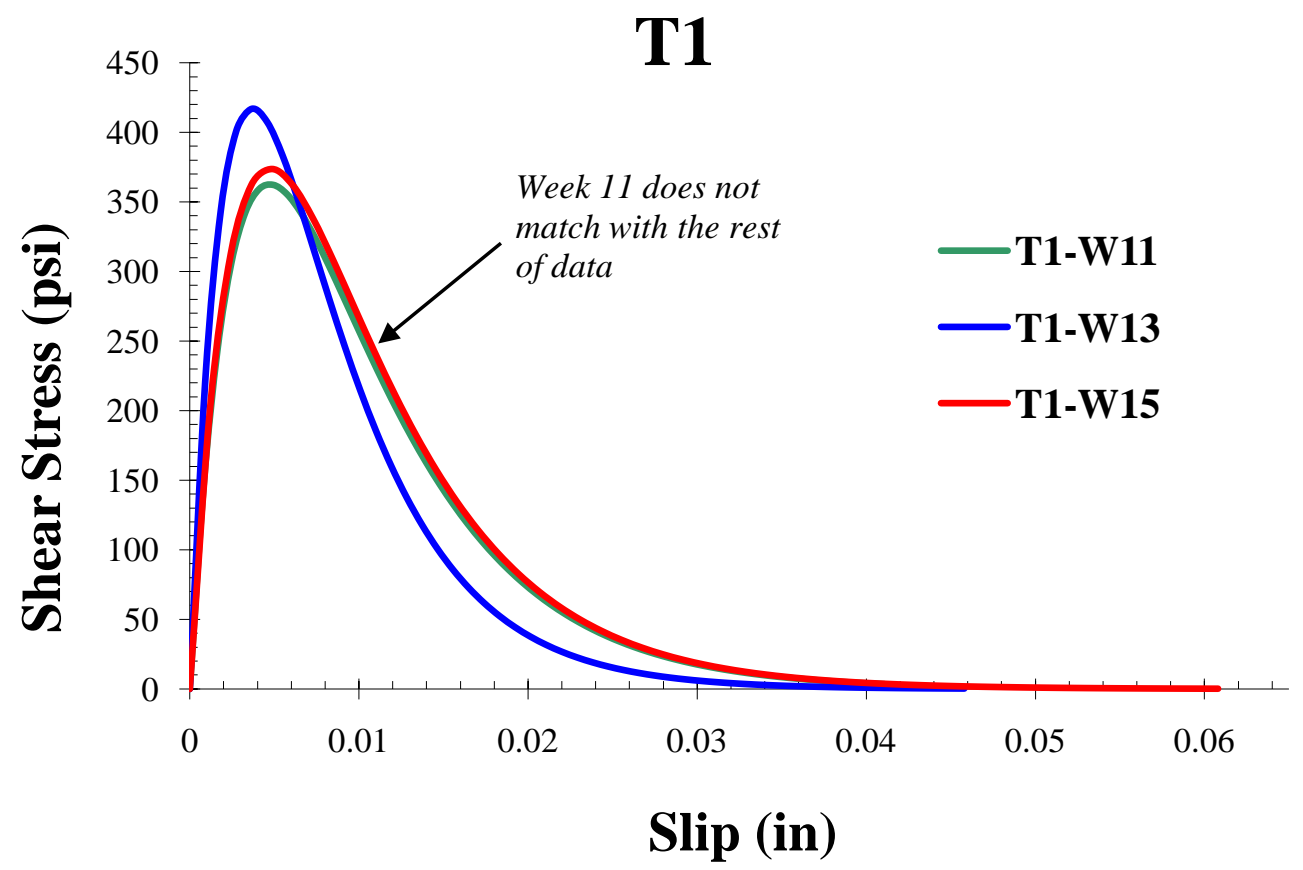

(a)

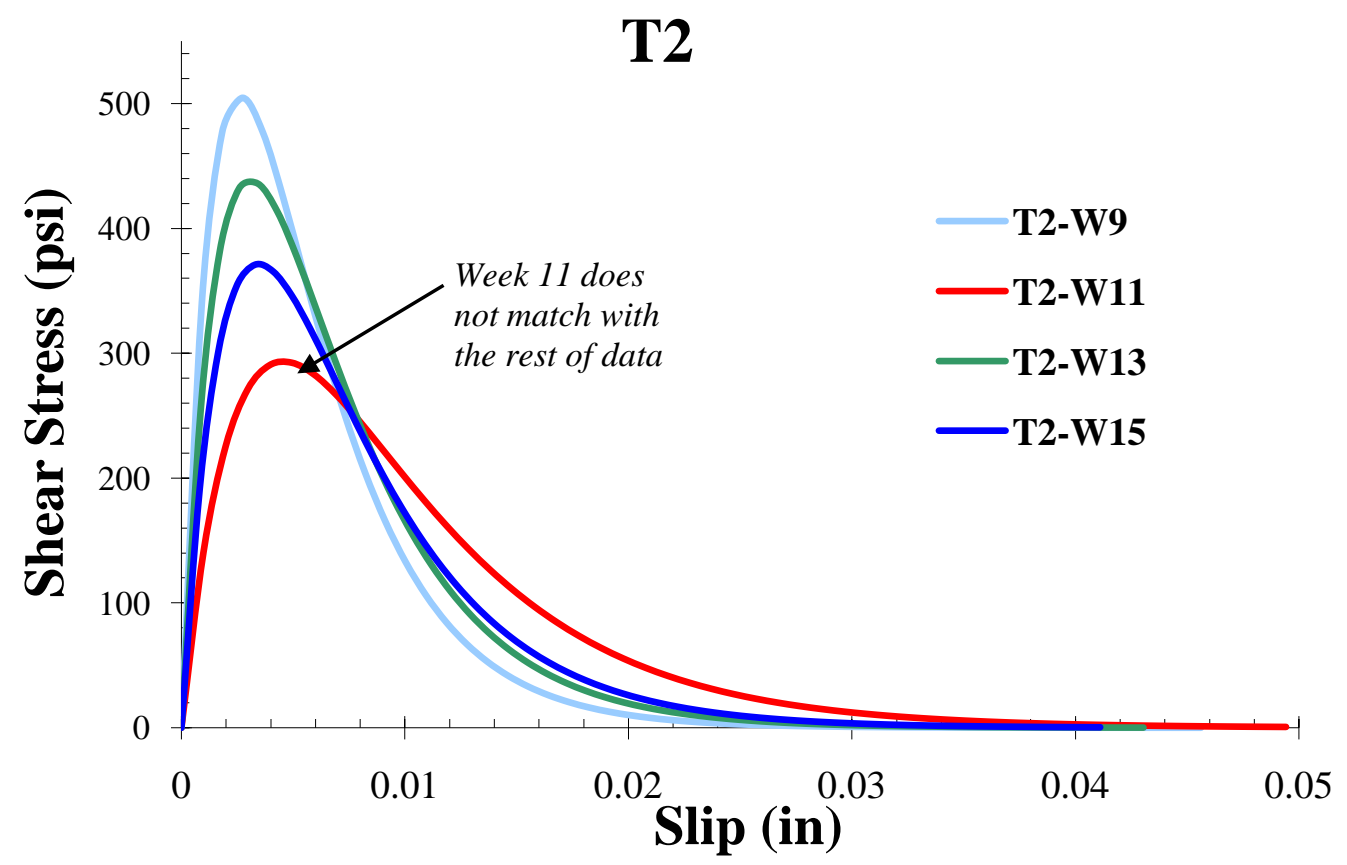

(b)

Figure 5-9 Traction-Separation Law; (a) T1, (b) T2 

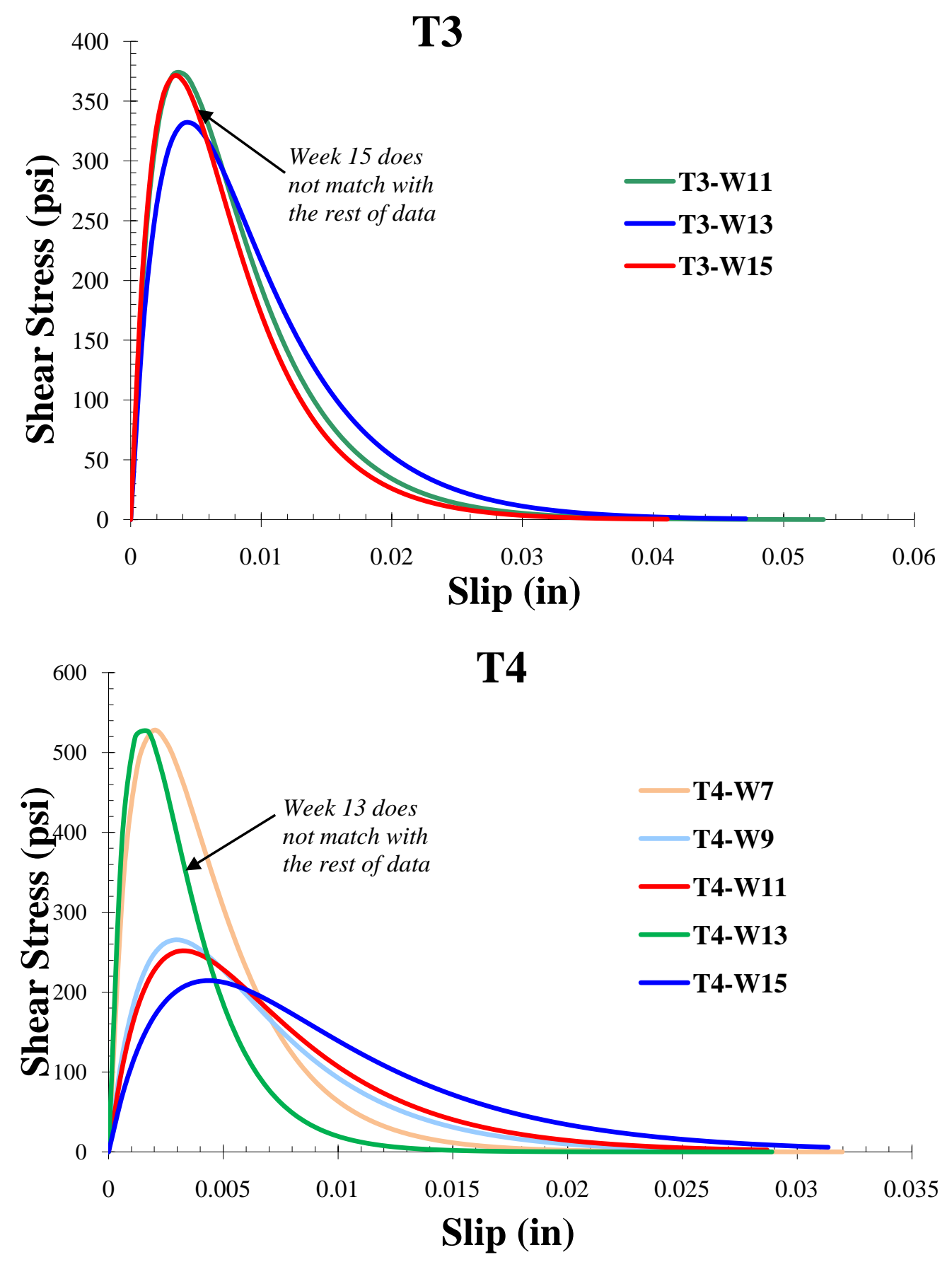

Figure 5-10 Traction-Separation Law; (a) T3, (b) T2

The relationship for each of the above curves can be obtained by substituting the empirical constants, A and B, from the tables below into Equation (4-12). 
Table 5-1 Empirical Constant, and the maximum Slip (T1)

\begin{tabular}{llll}
\hline $\mathbf{T 1}$ & & & \\
\hline & $\mathbf{1 1}$ & $\mathbf{1 3}$ & $\mathbf{1 5}$ \\
$\boldsymbol{A}$ & -2622.23 & -2488.66 & -2670.00 \\
$\boldsymbol{B}$ & -146.60 & -187.08 & -145.80 \\
$\boldsymbol{S}_{\max }$ & 0.041062 & 0.045796 & 0.060794 \\
\hline
\end{tabular}

Table 5-2 Empirical Constant, and the maximum Slip (T2)

\begin{tabular}{l|l|l|l|l}
\hline \multicolumn{5}{l}{ T2 } \\
\hline & $\mathbf{9}$ & $\mathbf{1 1}$ & $\mathbf{1 3}$ & $\mathbf{1 5}$ \\
$\boldsymbol{A}$ & -2305.85 & -2317.54 & -2328.01 & -2404.41 \\
$\boldsymbol{B}$ & -264.04 & -151.77 & -224.78 & -263.07 \\
$\boldsymbol{S}_{\max }$ & 0.045620 & 0.049422 & 0.043037 & 0.044434 \\
\hline
\end{tabular}

Table 5-3 Empirical Constant, and the maximum Slip (T3)

\begin{tabular}{l|l|l|l}
\hline \multicolumn{3}{l}{ T3 } & \multicolumn{3}{l}{} \\
\hline & $\mathbf{1 1}$ & $\mathbf{1 3}$ & $\mathbf{1 5}$ \\
$\boldsymbol{A}$ & -2354.96 & -2413.07 & -2264.67 \\
$\boldsymbol{B}$ & -187.87 & -158.67 & -201.31 \\
$\boldsymbol{S}_{\max }$ & 0.053040 & 0.047105 & 0.041096 \\
\hline
\end{tabular}

Table 5-4 Empirical Constant, and the maximum Slip (T4)

\begin{tabular}{l|l|l|l|l|l}
\hline T4 & \multicolumn{1}{l}{} \\
\hline & $\mathbf{9}$ & $\mathbf{1 1}$ & $\mathbf{1 3}$ & $\mathbf{1 5}$ \\
$\boldsymbol{A}$ & -2054.62 & -1777.15 & -1818.85 & -1777.78 & -1934.56 \\
$\boldsymbol{B}$ & -347.54 & -233.76 & -211.59 & -468.69 & -159.20 \\
$\boldsymbol{S}_{\max }$ & 0.031965 & 0.028714 & 0.028700 & 0.028900 & 0.031350 \\
\hline
\end{tabular}




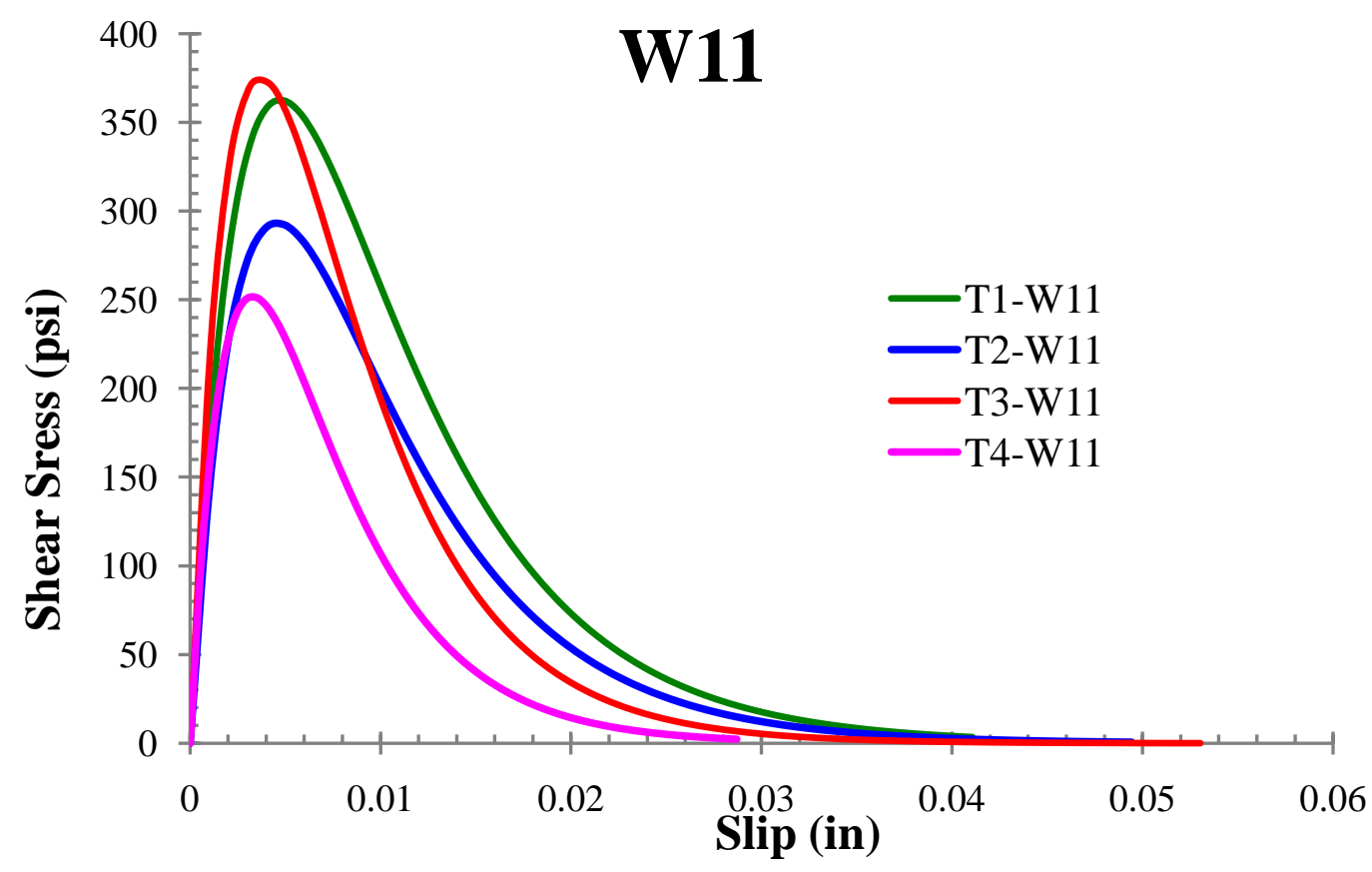

Figure 5-11 $\tau$-s Curves for various Temperatures at the end of Week 11

\subsection{Traditional Approach}

The traditional method of obtaining interfacial shear stress-slip relationship was fully explained in section 4.7.3. Stress and Strain are both obtained using strain distribution in CFRP layer. Figure 5-12 illustrates a schematic view of strain field along the FRP, assuming a linear distribution between each two successive strain gages.

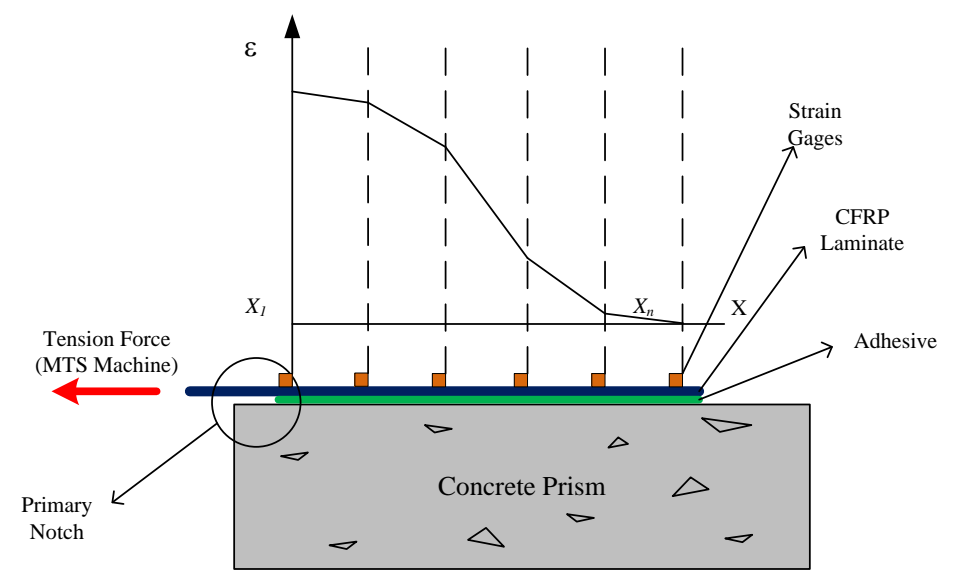

Figure 5-12 Typical strain distribution along the FRP

Since slip is calculated by integrating the strain field, its distribution is quadratic. Shear stress, however, is obtained using strain gradient between each two successive strain gages, and 
thus is constant. Strain distribution, along with shear stress, and slip distributions are schematically shown in Figure 5-13.

Three regions can be identified in the strain curve: completely debonded zone, stresstransfer zone, and perfectly bonded zone. At the debonded zone, the strain levels-off at a constant strain. This trend was observed in all specimens with the strain leveling- off at a threshold value.
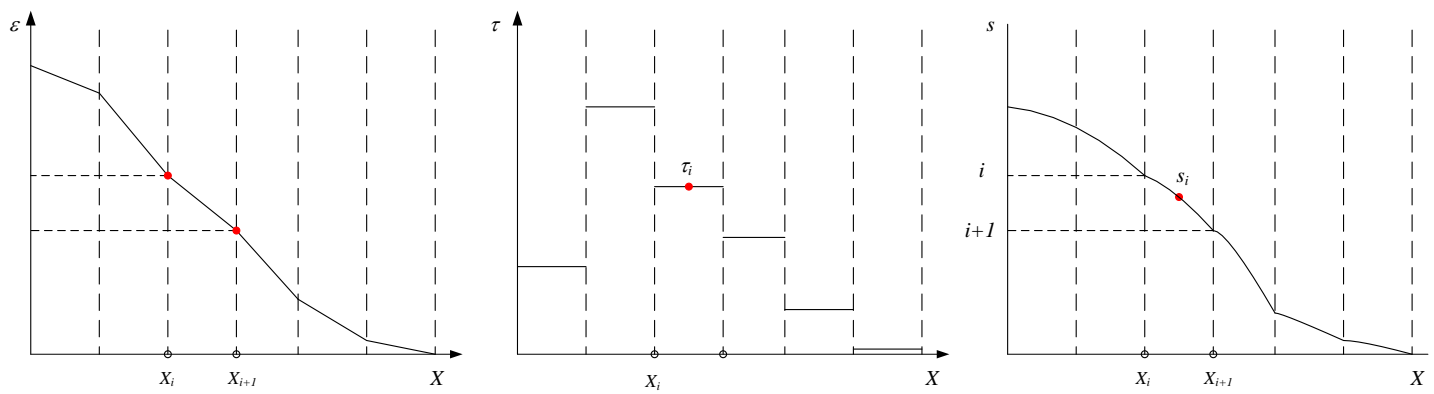

Figure 5-13 Typical Strain, Stress, and Slip Profiles for Different Load Levels

Shear Stress, and slip are obtained using equations (4-21) and (4-19), respectively, and the ERR is calculated using numerical integration (the area under $\tau$-s curves). In data reduction, the strain distribution after reaching $85-90 \%$ of the maximum load was disregarded because either nonsense jumps in the strain were observed or a negative value was recorded for the strain. The ERR obtained for different specimens using equation (2-20), the area under the curve obtained by J-integral, and the area under the curve obtained by the traditional method is presented in the table below:

Table 5-5 ERR (lbf/in)

\begin{tabular}{|c|c|c|c|c|}
\hline & & FORMULA & J-INTEGRAL & TRADITIONAL \\
\hline \multirow{8}{*}{ T1 } & CONTROL & 6.43 & - & 6.22 \\
\hline & w3 & 5.48 & - & 5.27 \\
\hline & W5 & 5.28 & - & 4.68 \\
\hline & W7 & 5.23 & - & 5.56 \\
\hline & W9 & 5.19 & - & 5.08 \\
\hline & W11 & 5.29 & 4.92 & 4.26 \\
\hline & W13 & 4.94 & 4.45 & 4.99 \\
\hline & W15 & 4.92 & 5.13 & 5.14 \\
\hline
\end{tabular}


Table 5-6 ERR (lbf/in)

\begin{tabular}{|c|c|c|c|c|}
\hline & & FORMULA & J-INTEGRAL & TRADITIONAL \\
\hline \multirow{8}{*}{$\mathbf{T 2}$} & CONTROL & 6.43 & - & 6.22 \\
\hline & W3 & 5.74 & - & 5.63 \\
\hline & W5 & 5.22 & - & 5.09 \\
\hline & W7 & 5.13 & - & 5.66 \\
\hline & W9 & 4.54 & 3.83 & 4.38 \\
\hline & W11 & 4.04 & 3.86 & 3.92 \\
\hline & W13 & 4.24 & 3.89 & 3.57 \\
\hline & W15 & 4.28 & 4.16 & 3.51 \\
\hline
\end{tabular}

Table 5-7 ERR (lbf/in)

\begin{tabular}{c|c|c|c|c}
\hline \multicolumn{1}{c|}{} & FORMULA & J-INTEGRAL & TRADITIONAL \\
\hline \multirow{6}{*}{} & CONTROL & 6.43 & - & 6.22 \\
& W3 & 4.30 & - & 5.08 \\
& W5 & 4.80 & - & 4.58 \\
T3 & W7 & 3.86 & - & 3.56 \\
& W9 & 3.80 & 3.78 & 3.03 \\
& W11 & 3.67 & 3.99 & 3.06 \\
& W13 & 3.86 & 4.18 & 4.17 \\
& W15 & 3.62 & 3.69 & 3.95 \\
\hline
\end{tabular}

Table 5-8 ERR (lbf/in)

\begin{tabular}{c|c|c|c|c}
\hline \multicolumn{1}{c|}{} & FORMULA & J-INTEGRAL & TRADITIONAL \\
\hline \multirow{5}{*}{ T4 } & CONTROL & 6.43 & - & 6.22 \\
& W3 & 4.18 & - & 5.56 \\
& W5 & 4.37 & 4.45 & 4.01 \\
& W7 & 3.09 & 3.04 & 2.31 \\
& W9 & 2.69 & 2.27 & 2.46 \\
& W11 & 2.59 & 2.37 & 2.12 \\
& W13 & 2.48 & 2.27 & 2.99 \\
& W15 & 2.39 & 2.66 & 2.37 \\
\hline
\end{tabular}

As can be seen from the above tables, ERR calculated from the traditional method is generally lower than that of J-integral which can be attributed to disregarding the strain data after 
reaching to the load level were strain did not make any sense. Figure 5-14 through Figure 5-21 depict a comparison between the $\tau$-s curves obtained from both traditional and J-integral method. Generally the maximum shear stress is higher for the traditional approach and the maximum slip is lower, making the area under the two curves (ERR) close together.

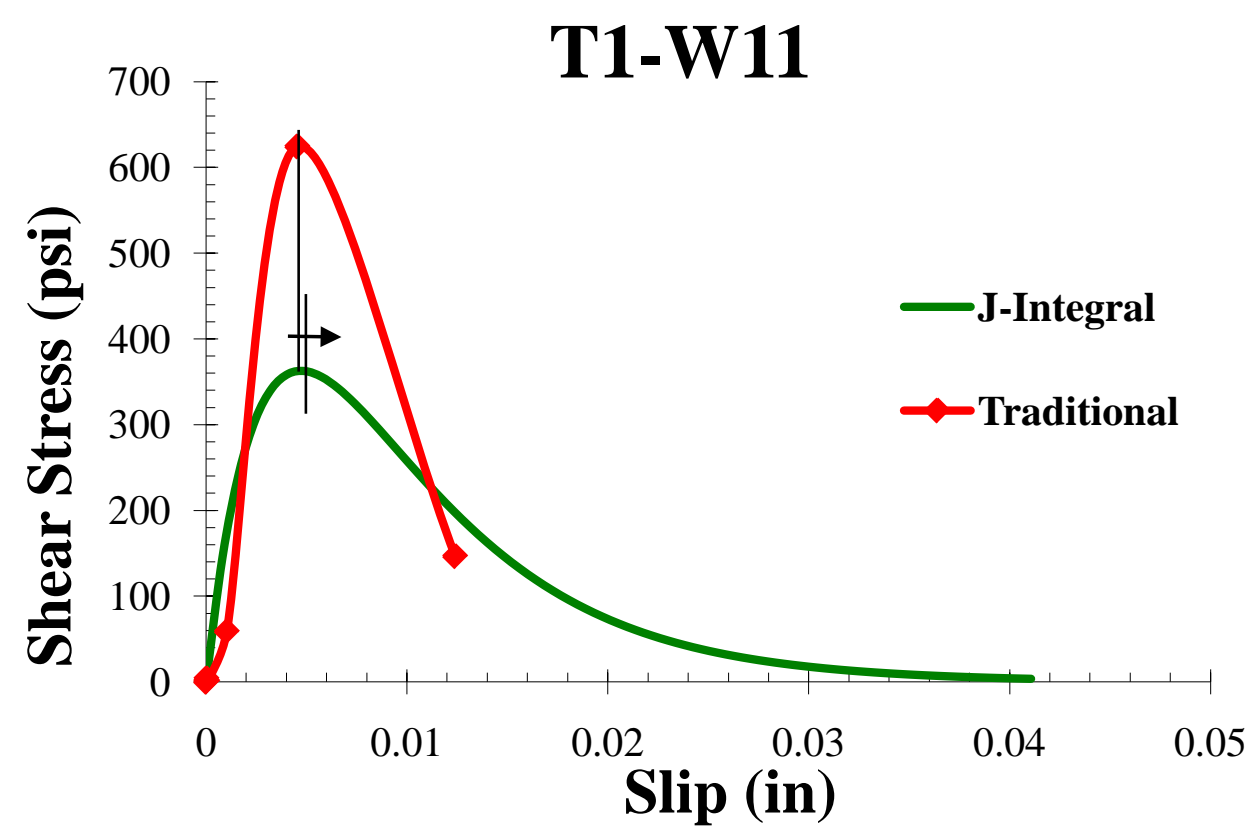

Figure 5-14 Traction-Separation Law (J-integral vs. Traditional Approach) 


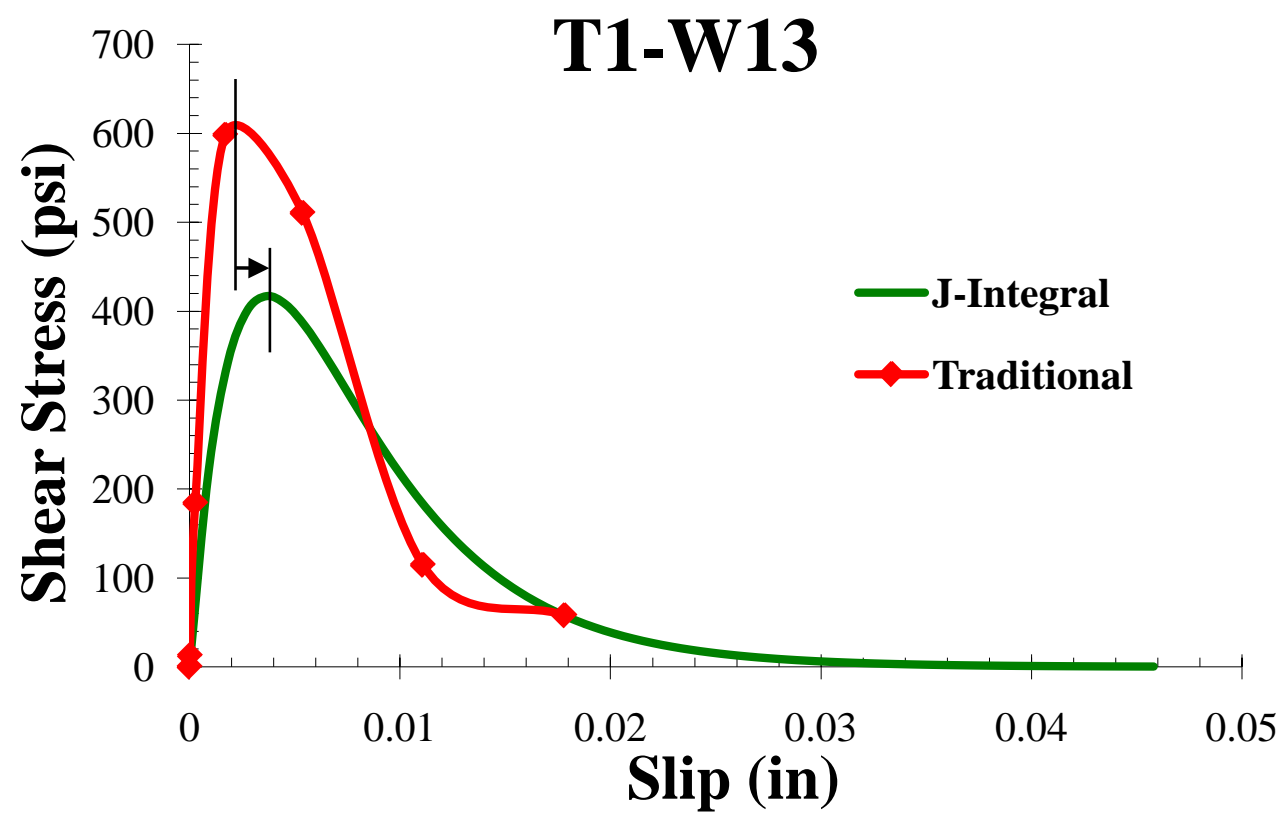

Figure 5-15 Traction-Separation Law (J-integral vs. Traditional Approach)

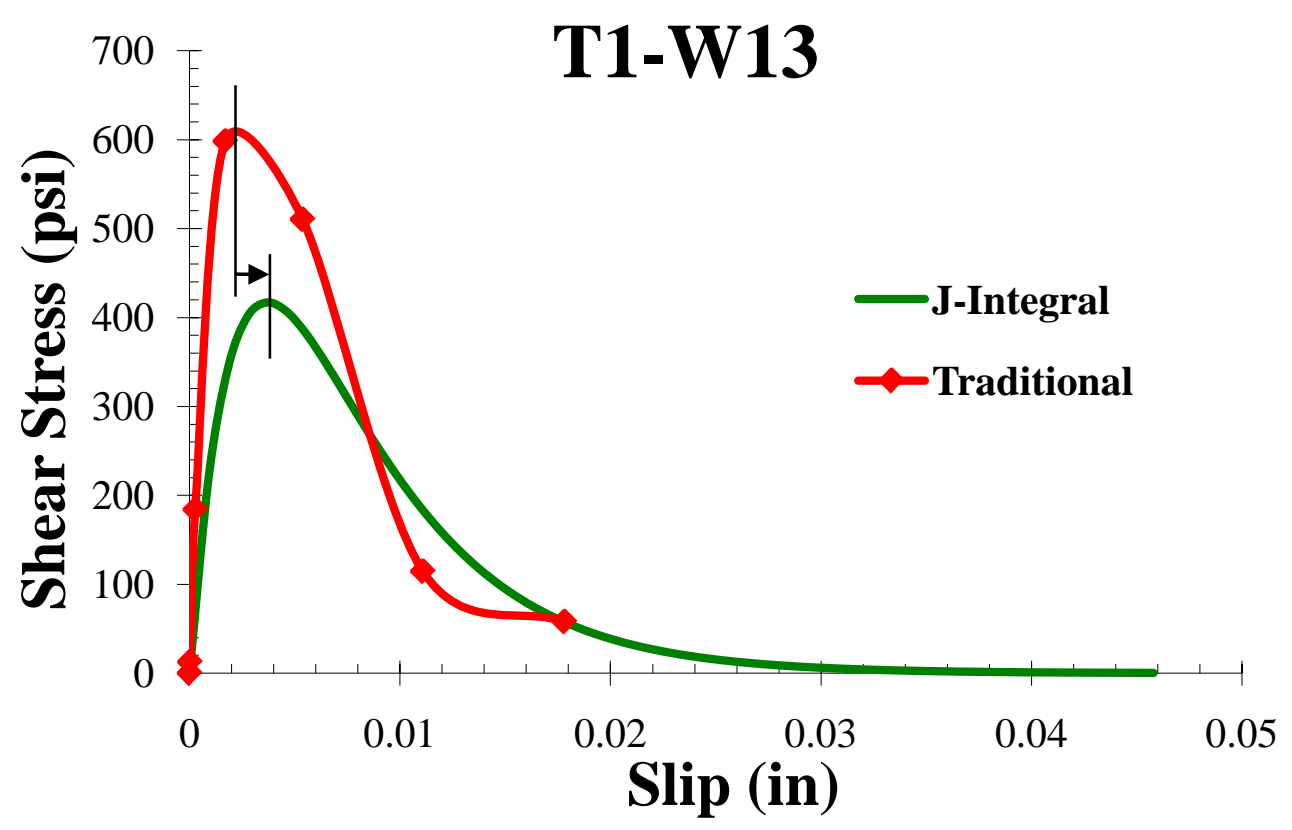

Figure 5-16 Traction-Separation Law (J-integral vs. Traditional Approach) 


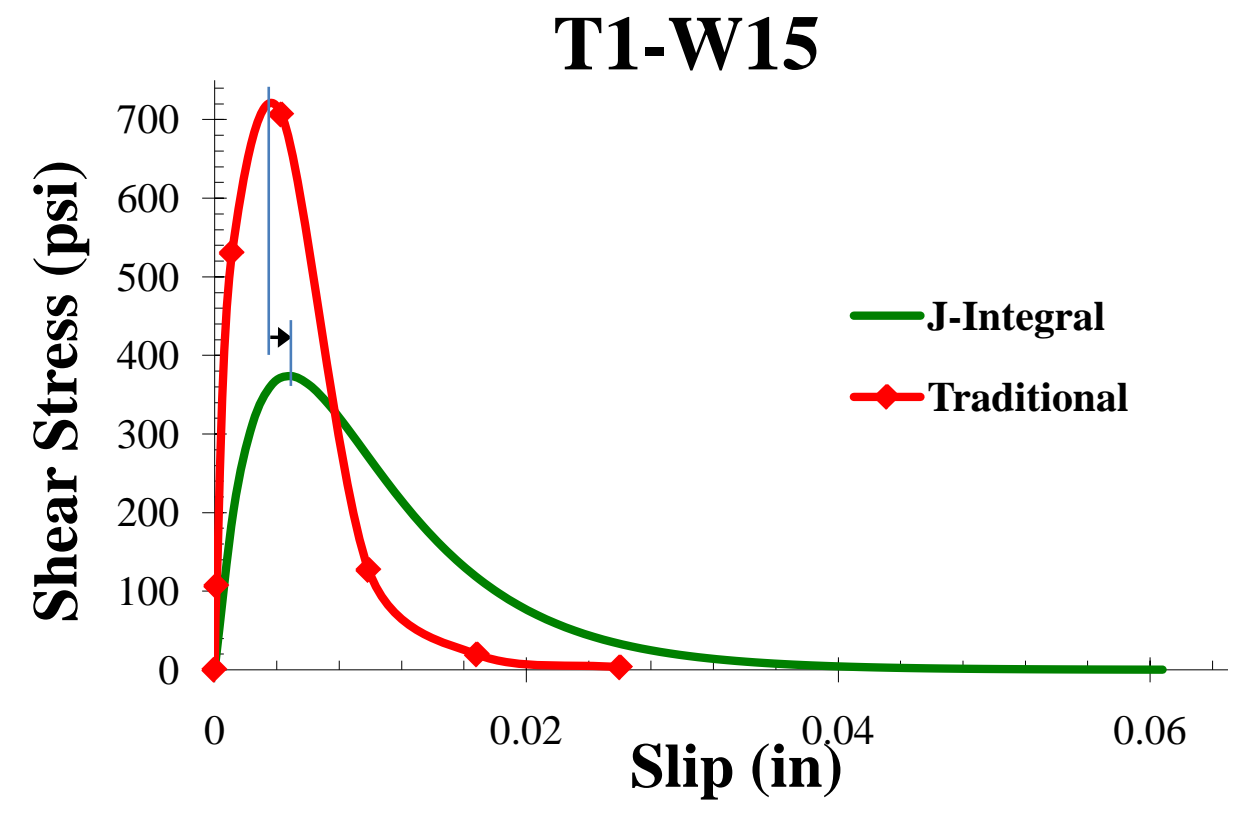

Figure 5-17 Traction-Separation Law (J-integral vs. Traditional Approach)

\section{T1-W15}

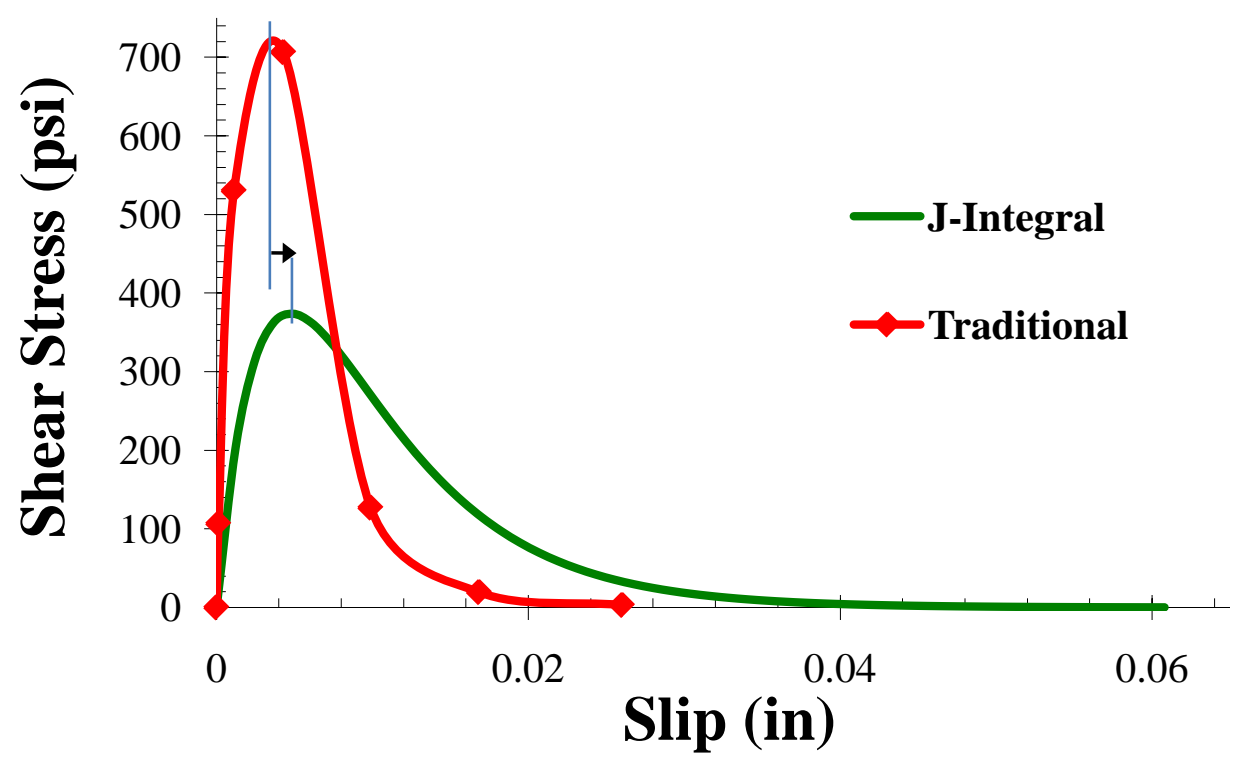

Figure 5-18 Traction-Separation Law (J-integral vs. Traditional Approach) 


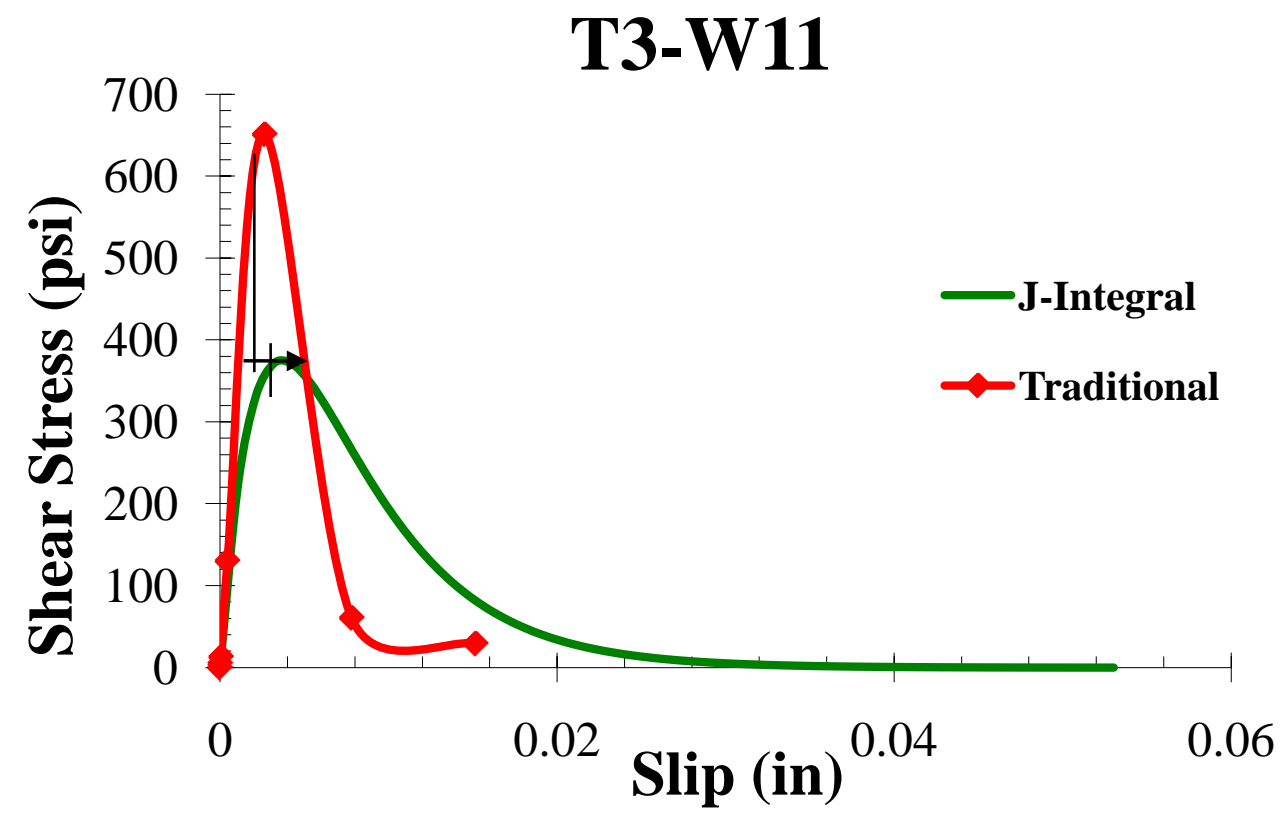

Figure 5-19 Traction-Separation Law (J-integral vs. Traditional Approach)

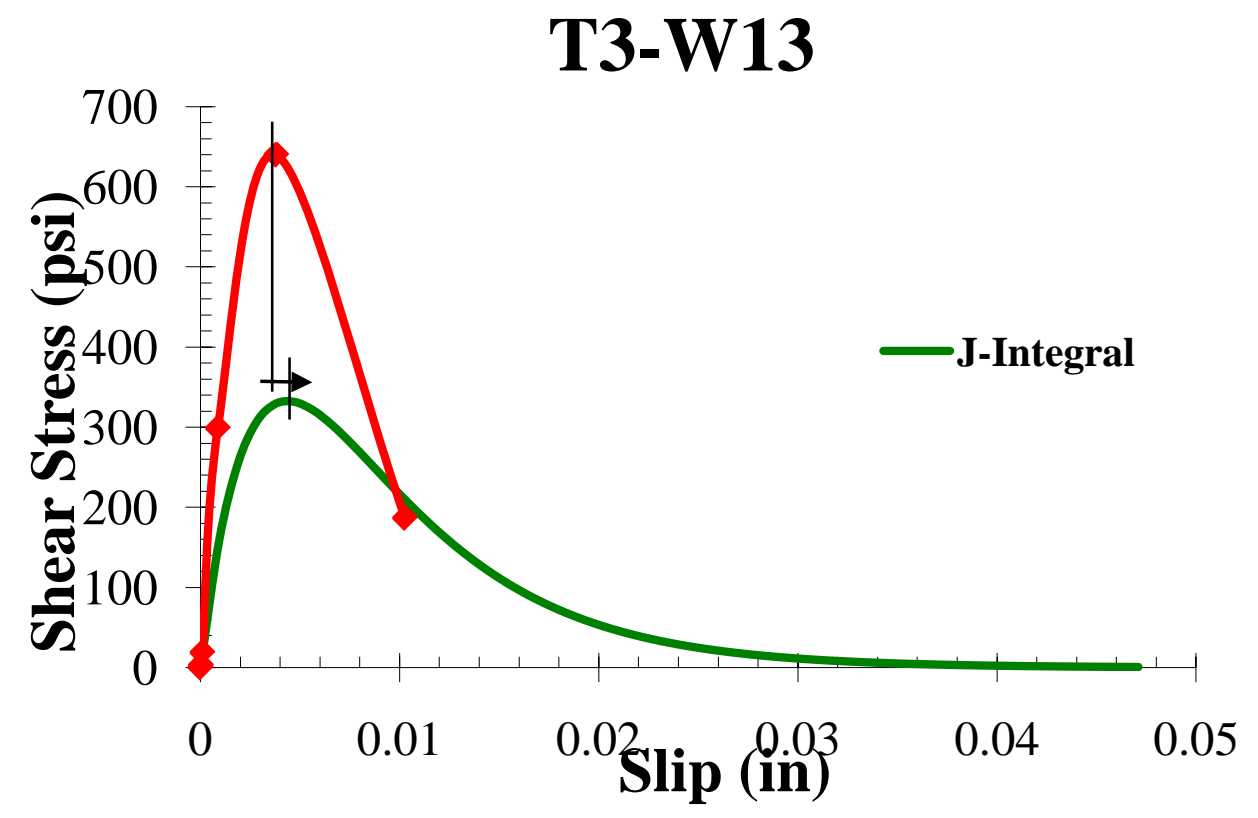

Figure 5-20 Traction-Separation Law (J-integral vs. Traditional Approach) 


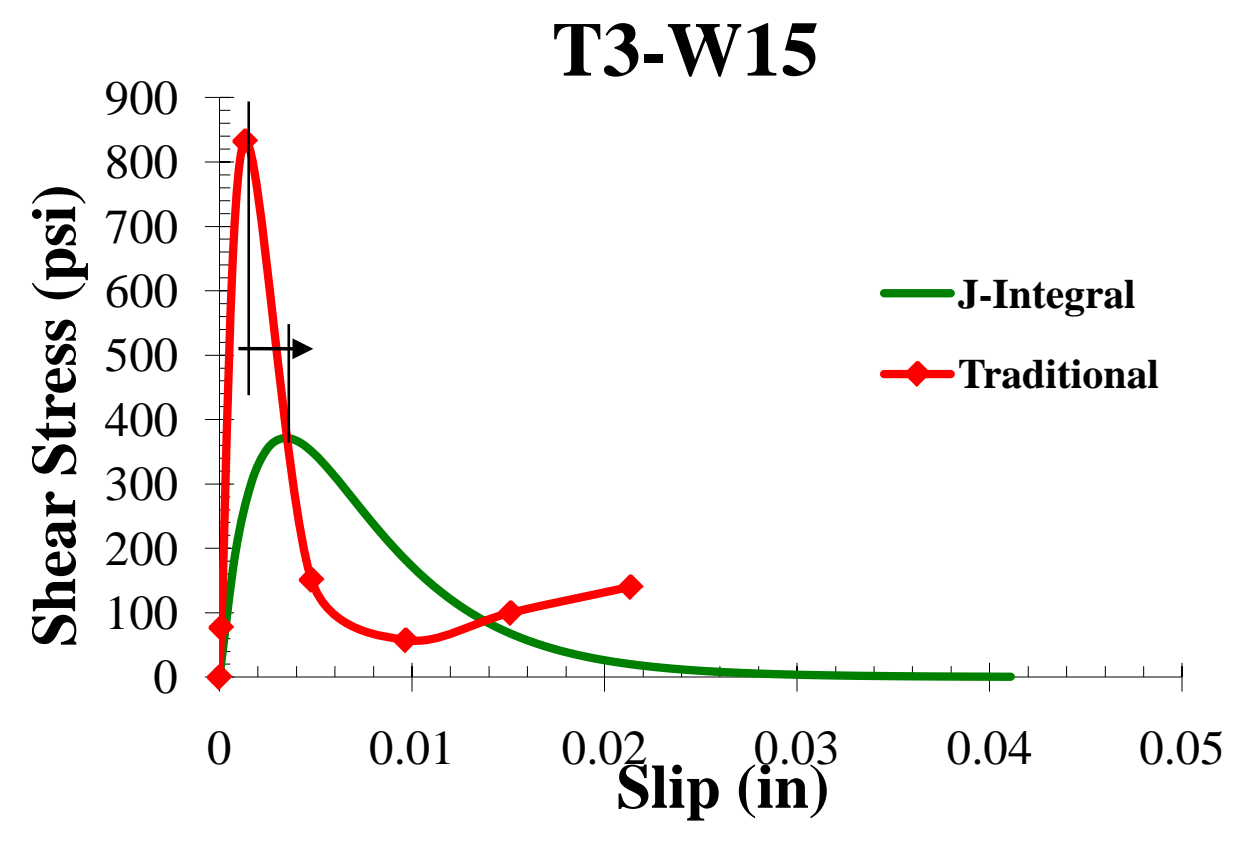

Figure 5-21 Traction-Separation Law (J-integral vs. Traditional Approach)

\subsection{Failure Mode}

With the increase of the applied load, debonding initiated from the predefined notch followed by a complete debonding of the FRP laminate from the concrete substrate. Upon failure, a thin layer of concrete was attached to the FRP, indicating a cohesive failure as shown in Figure 5-22. As it was expected, the thickness of the concrete attached to the FRP decreased with the increase of conditioning showing a trend of shifting toward the adhesive failure. Similar observations were reported by Ouyang and Wan (2008a), and Davalos et al. (2008) for Mode-I, and by Subramaniam et al. (2008) for Mode II failure. This observation is verified by the results of the optical microscopy (See 4.5.5). The failure was abrupt and the FRP laminate was completely separated from the concrete substrate. 


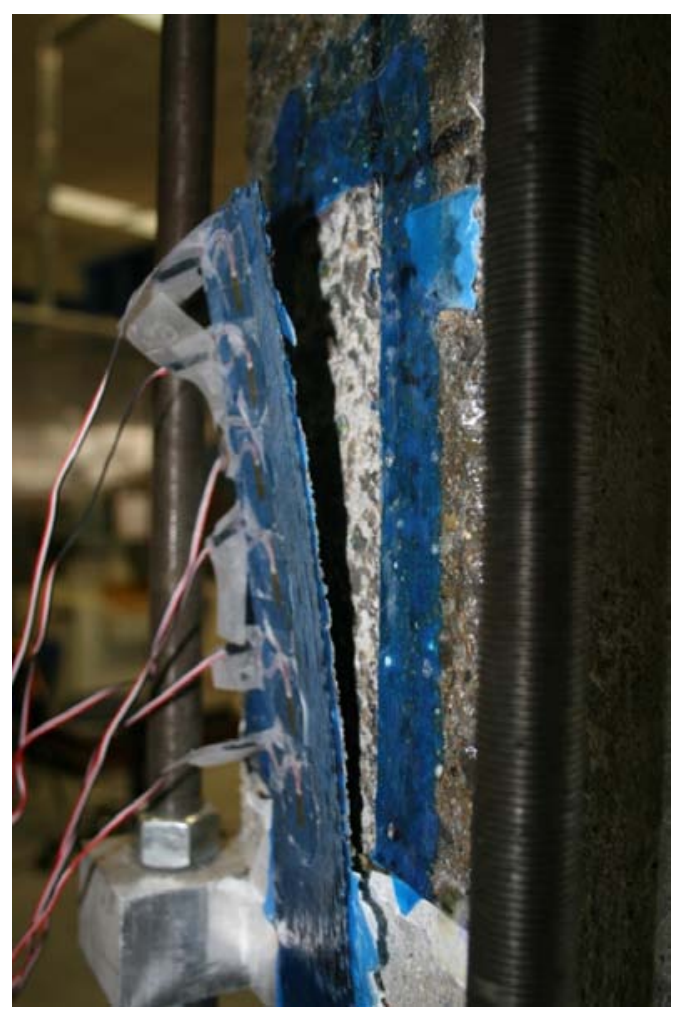

(a)

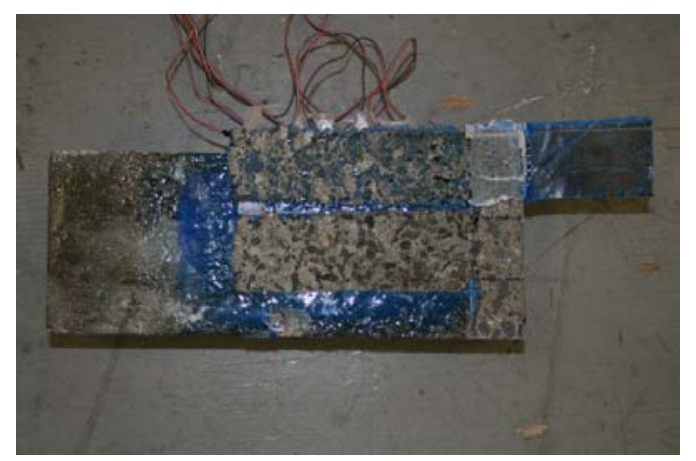

(b)

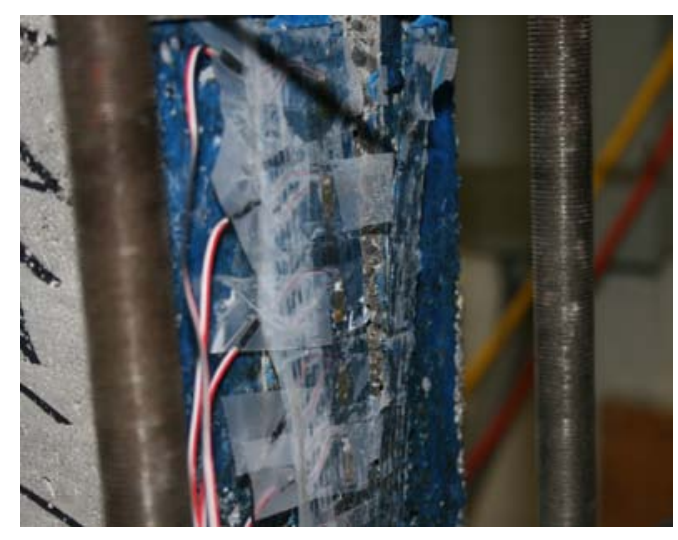

(c)

Figure 5-22 (a) Abrupt Failure, (b) Delaminated FRP, and the Concrete Counterpart, (c) Inappropriate Failure

In case the FRP was not gripped appropriately or it was misaligned with the actuator, failure could occur within the FRP in a way that after final failure parts of FRP were still attached to the concrete (Figure 5-22 (a) Abrupt Failure, (b) Delaminated FRP, and the Concrete Counterpart, (c) Inappropriate FailureFigure 5-22 (c)).

For some samples tested after the 13th week of immersion in water, the failure was completely adhesive with almost no concrete attached to the FRP. Ouyang and Wan (2008a) showed that the thickness of the concrete attached to the FRP after delamination is related to the relative humidity of the interface, which is in agreement with findings of this research.

In most specimens, when the load was about $60 \%$ of the maximum load, some noises could be heard from the specimen. 


\subsection{Optical Microscopy}

Image processing of some representative fractured laminates was performed to obtain a qualitative understanding about the effects of temperature and water immersion on the nature of cohesive or adhesive failure modes by measuring the areas of concrete, epoxy, and fibers on the delaminated CFRP. Optical images were recorded as explained in section 4.5.5, and image processing was performed using Image-Pro Plus 6. Figure 5-23 shows two representative images from T1-W13 samples. Figure 5-29 to Figure 5-38 show the epoxy/concrete/fiber proportions for each segment of each individual specimen. There was not any specific trend observed in these percentiles along the length of an individual sample. However, the blue area which represented epoxy, increased with increasing the temperature and exposure period. In other words, the results of the optical microscopy confirm shifting from cohesive toward adhesive failure.

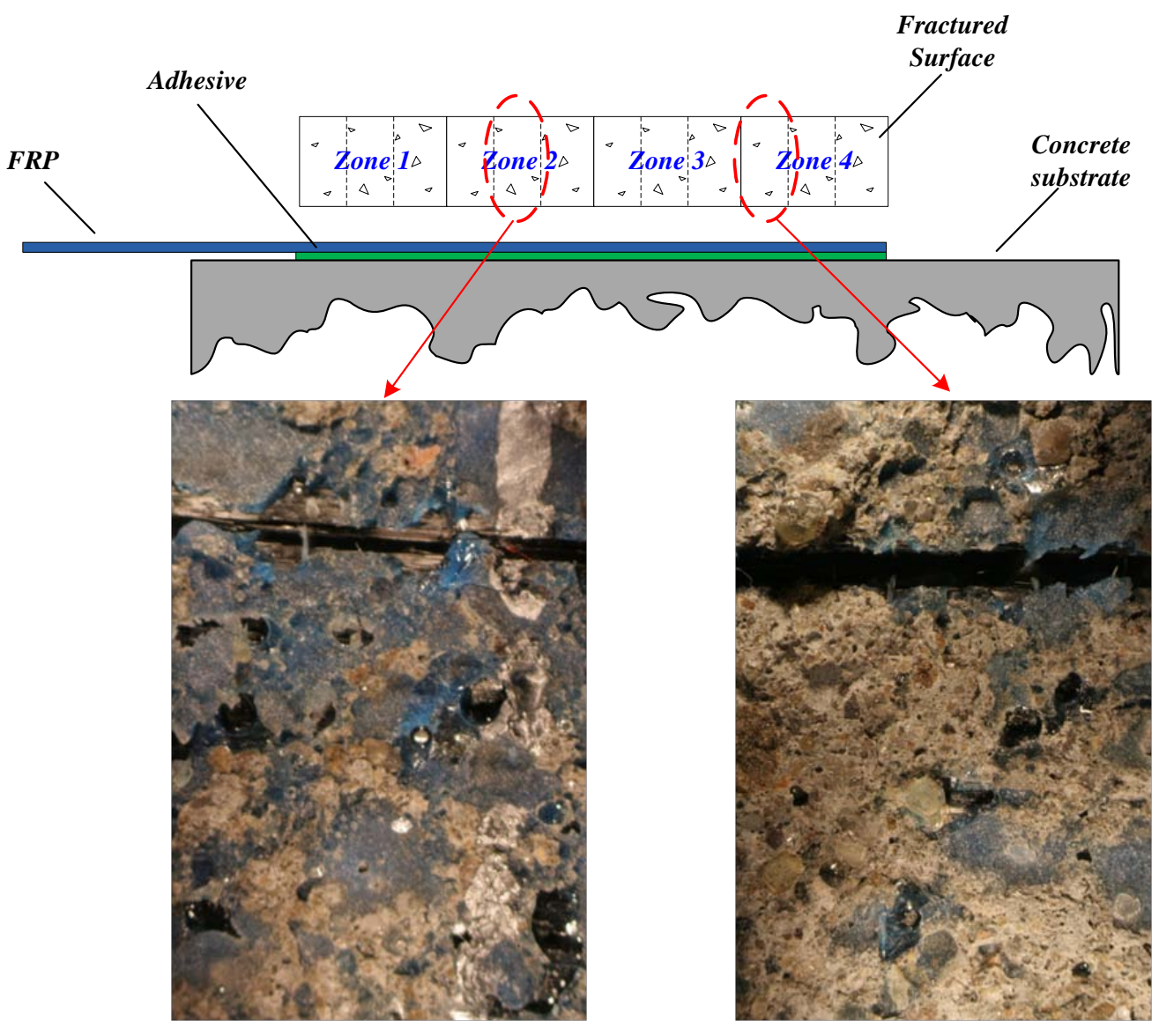

(a)

(b)

Figure 5-23 T1-W13; (a) Zone 2, (b) Zone 4

The relative fractured surfaces (RFS) are shown in 5-24 to Figure 5-26 for T1, T2, and T3 specimens immersed in water for 11,13 , and 15 weeks along with the control specimen. 


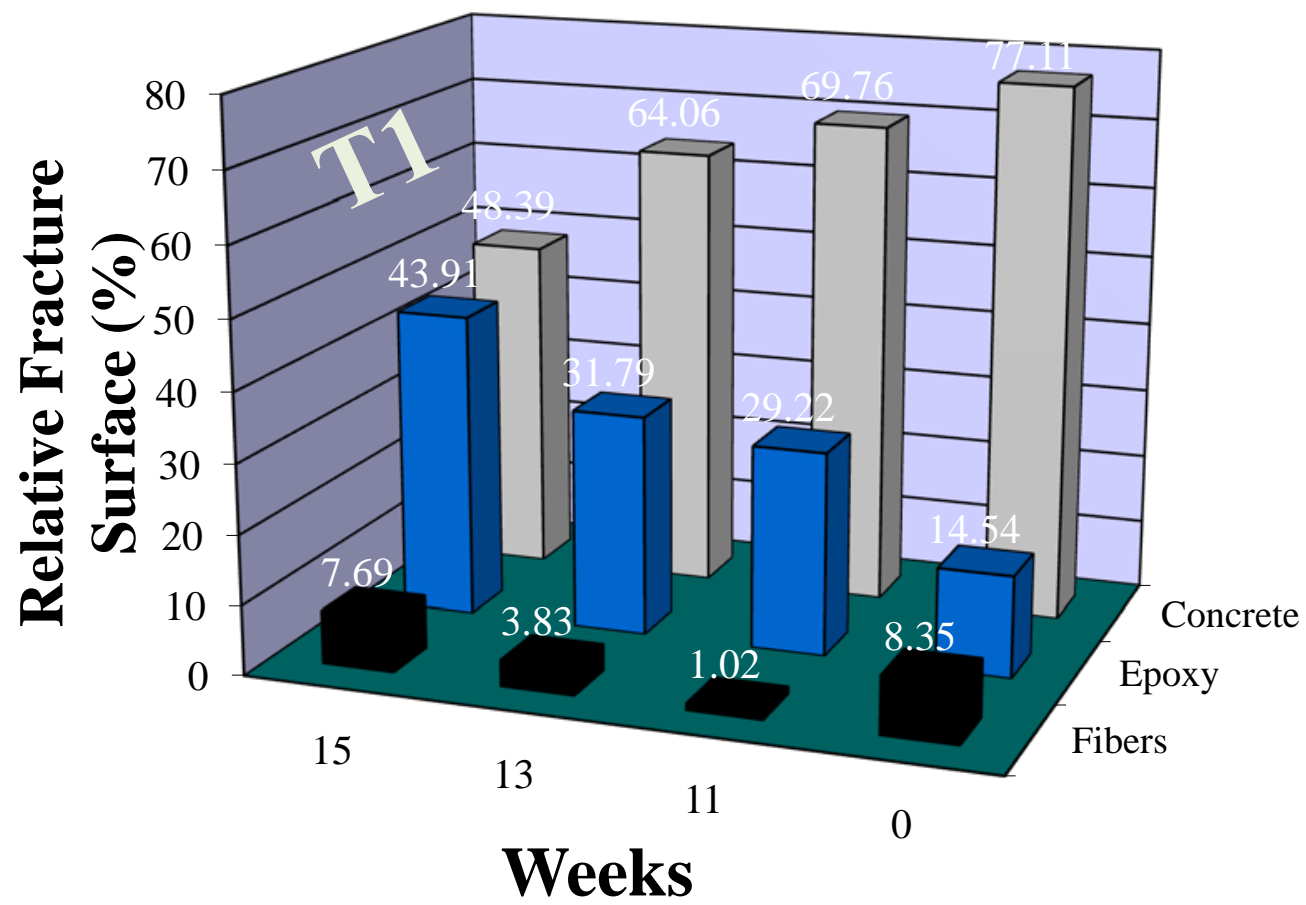

5-24 Relative Fracture Surface upon Images Processing (T1, Weeks 11-13-15, and control)

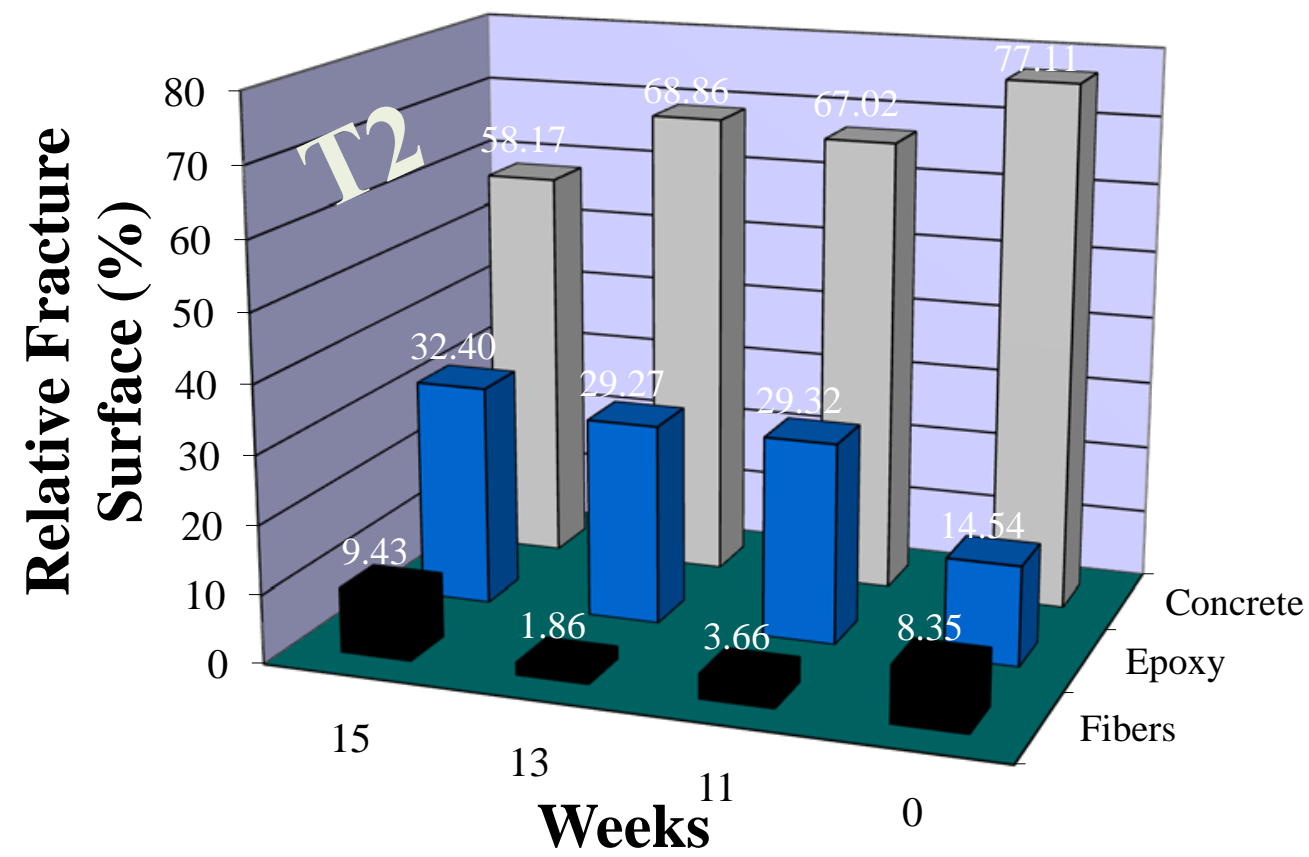

Figure 5-25 Relative Fracture Surface upon Images Processing (T2, Weeks 11-13-15 and control) 


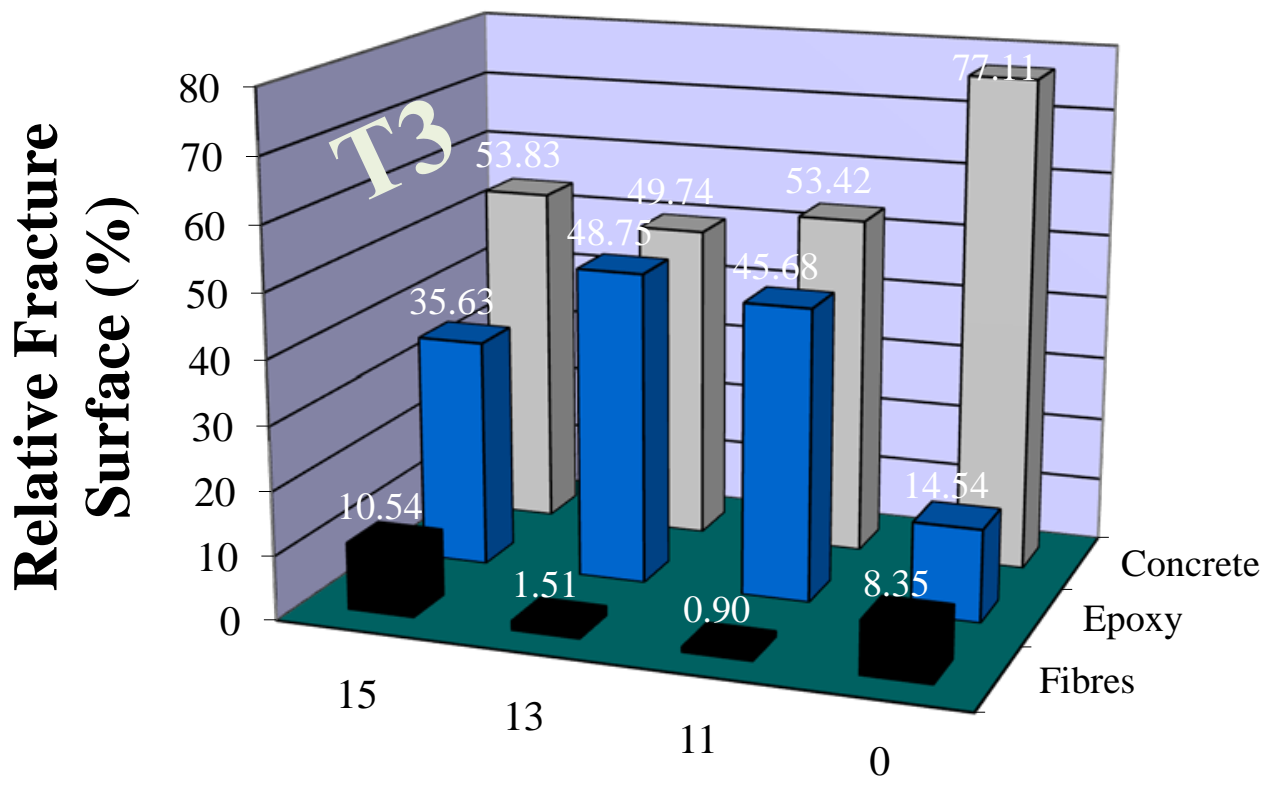

Weeks

Figure 5-26 Relative Fracture Surface upon Images Processing (T3, Weeks 11-13-15 and control)

Figure 5-26 illustrates failure levels over the period of 11 to 15 weeks. During this period of continuous immersion in water, the cohesive and adhesive failure proportions remain relatively constant for the specimens immersed in T3 water. Samples conditioned in T1 and T2 temperatures reveal a relatively significant drop in the cohesive failure as observed from week 13 to week 15 . However, the C/A failure ratio remains constant for week 11 and week 13,

The percentile shown in 5-24 to Figure 5-26 should not be assumed to be a definite number; since image processing is performed using operator's visual-estimation of the colors, and two different operators may come up with two different numbers. As a matter of fact, this information is rather qualitative, yet is presented in quantitative form.

Figure 5-27 shows the RFS for T2 specimens from the week 3 to week 15 water immersion. As hypothesized, concrete residue on the fractured laminates decreased over time by $25 \%$. 


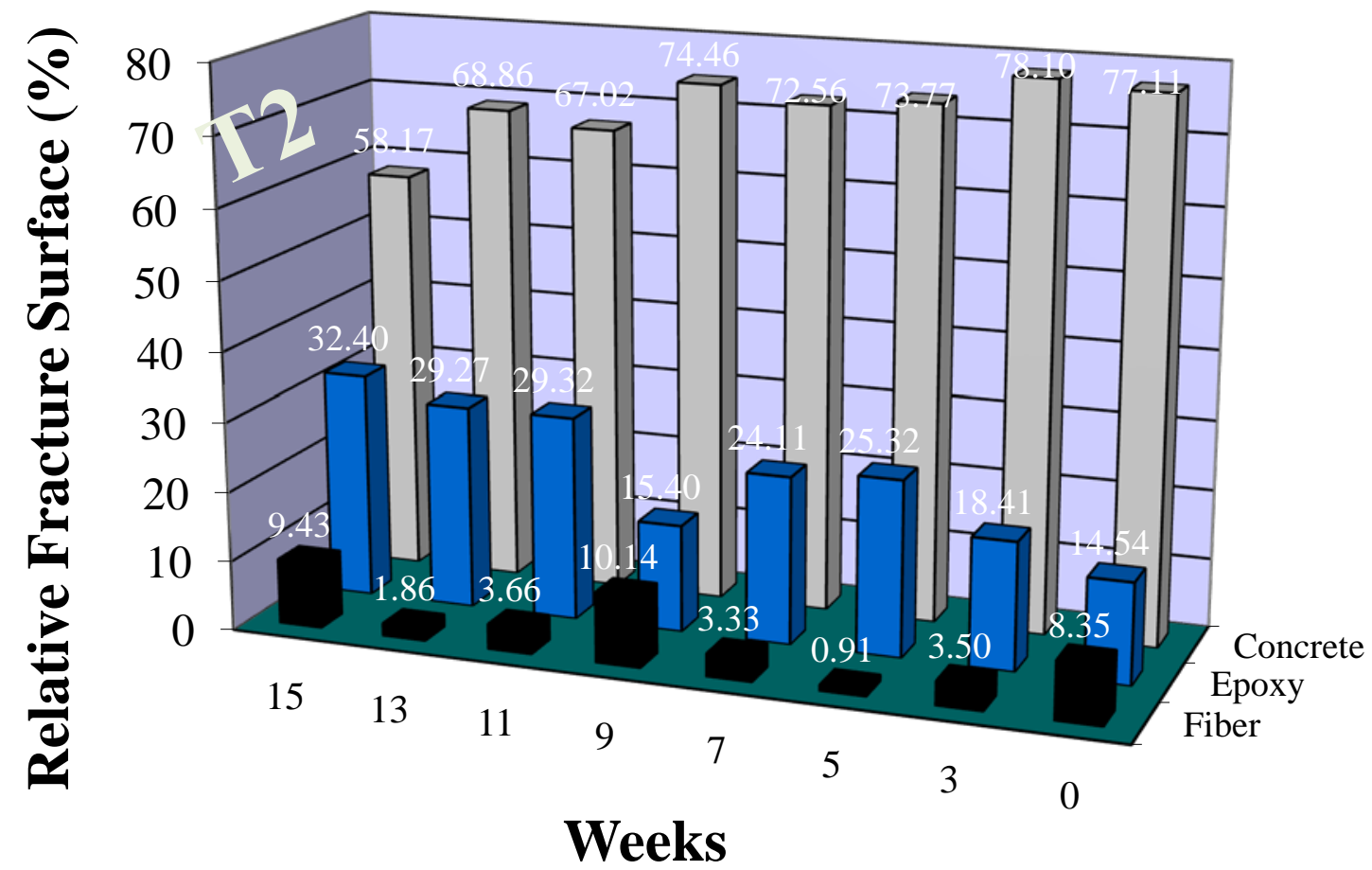

Figure 5-27 Relative Fracture Surface upon Images Processing (T2)

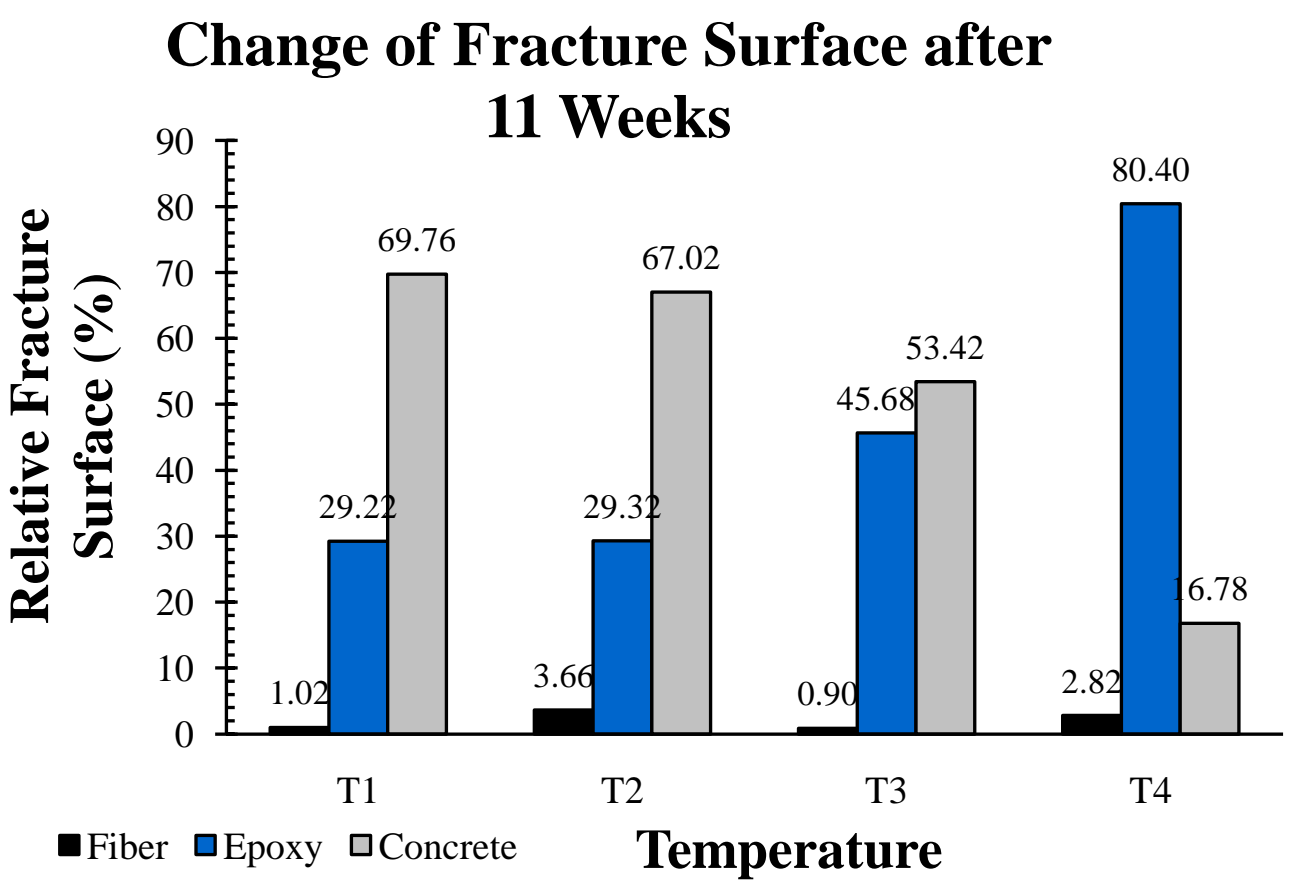

Figure 5-28 Change of the Fracture Surface after 11 weeks for different tempearaures 
Figure 5-28 gives a clear demonstration of how the fracture inherent shifts from cohesive to adhesive with increasing the temperature with a decrease of about $23 \%$ residual concrete on the fractured surface by increasing the temperature from T1 to T3.

\begin{tabular}{|c|c|c|c|}
\hline$D_{\Delta}^{D}$ & $\Delta^{D}$ & $D_{\Delta}^{D}$ & $D_{\Delta}^{D}$ \\
\hline Fibers: 2.22 & Fibers: 1.02 & Fibers: $\mathbf{0 . 6 6}$ & ${ }^{\triangleleft}$ Fibers: 0.16 \\
\hline Epoxy: 24.80 & Eроху: 30.49 & Epoxy: 31.69 & Eроху: 29.92 \\
\hline Concrete: 72.98 & Concrete: 68.49 & Concrete: 67.65 & Concrete: 69.92 \\
\hline$\Delta$ & $T 1-$ & $W_{411}$ & $\Delta$ \\
\hline
\end{tabular}

(a)

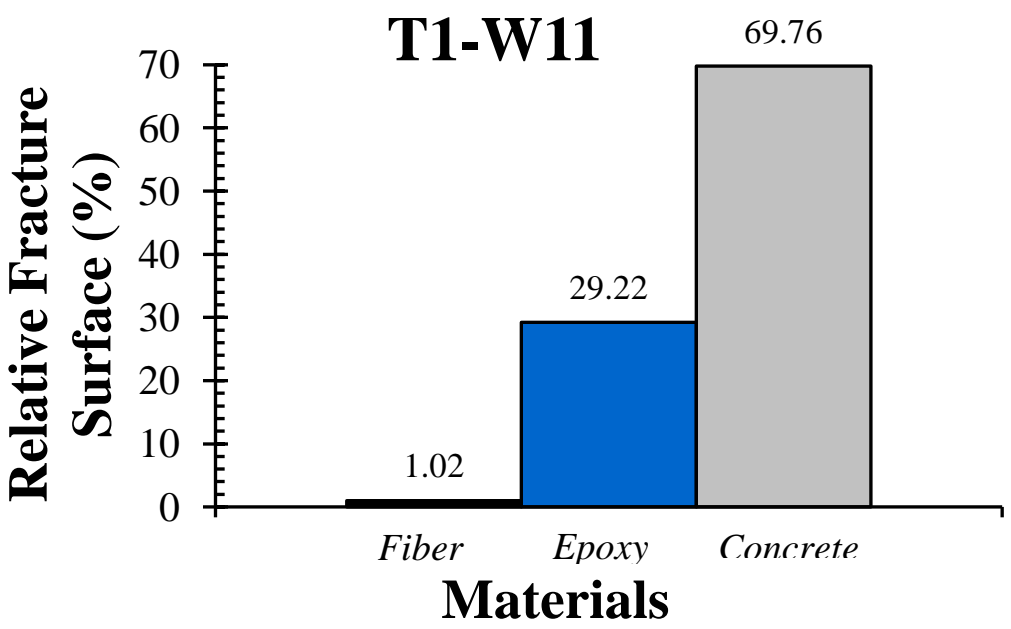

(b)

Figure 5-29 


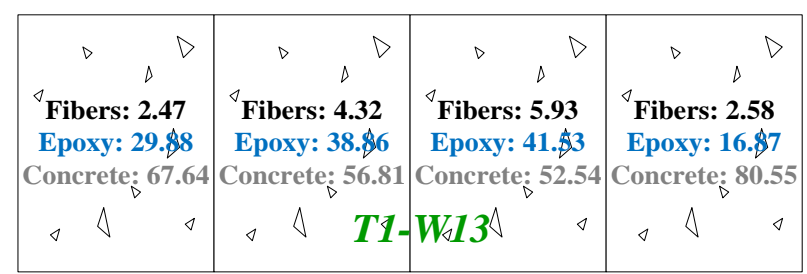

(a)

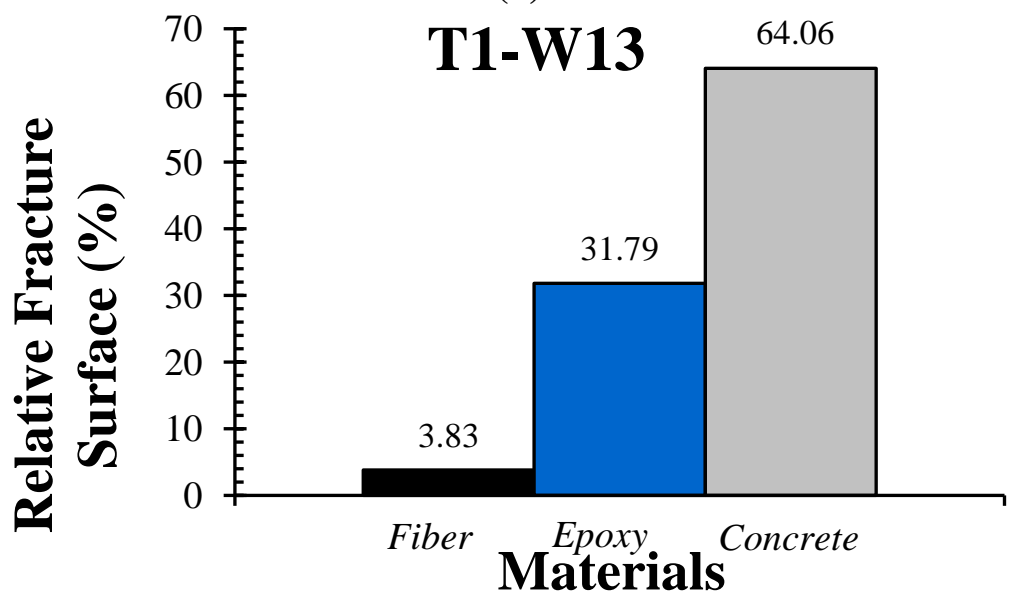

(b)

Figure 5-30

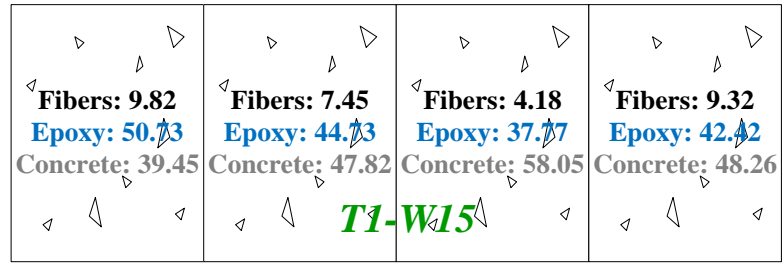

(a)

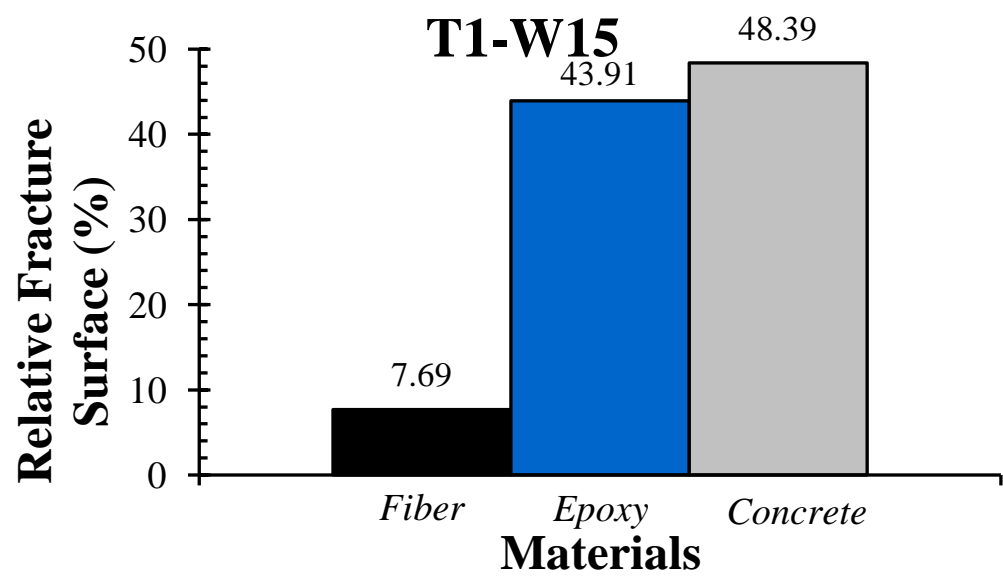

(b)

Figure 5-31 


\begin{tabular}{|c|c|c|c|}
\hline$\triangleright \quad D$ & $\triangleright \quad D$ & $D$ & $D$ \\
\hline${ }^{\triangleleft}$ Fibers: 3.15 & ${ }^{\triangleleft}$ Fibers: 1.75 & ${ }^{\triangleleft}$ Fibers: 1.26 & ${ }^{\triangleleft}$ Fibers: 1.30 \\
\hline Epoxy: 25.\$1 & Epoxy: $30 . \$ 0$ & Epoxy: $32 . \$ 1$ & Epoxy: $28 . \$ 8$ \\
\hline Concrete: 70.95 & Concrete: 67.75 & Concrete: 66.63 & Concrete: 70.13 \\
\hline$\triangleleft \Delta \triangleleft$ & $\triangle T 2$ & W/13 & $\triangleleft \Delta$ \\
\hline
\end{tabular}

(a)

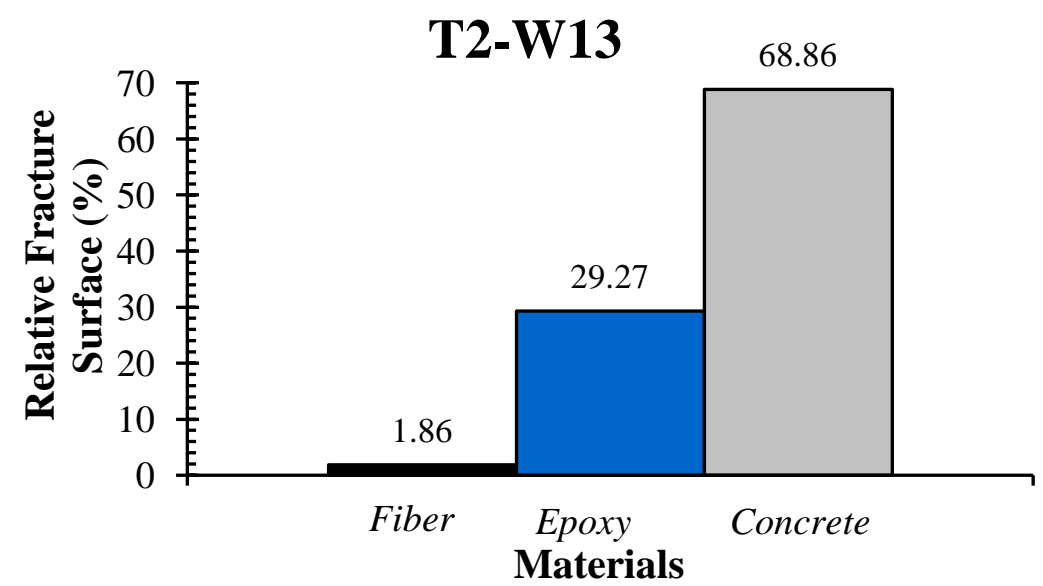

(b)

Figure 5-32

\begin{tabular}{|c|c|c|c|}
\hline$\triangleright \quad \nabla$ & $D$ & $D^{D}$ & $D$ \\
\hline Fibers: 13.50 & Fibers: 11.21 & ${ }^{\natural}$ Fibers: 7.14 & ${ }^{\natural}$ Fibers: 5.88 \\
\hline Epoxy: $30 . \$ 0$ & Epoxy: 34.63 & Epoxy: 40.\$5 & Epoxy: 23.91 \\
\hline Concrete: 56.00 & Concrete: 54.16 & Concrete: 52.31 & Concrete: 70.22 \\
\hline$\Delta$ & $T 2$ & Wis & $\triangleleft \quad \Delta$ \\
\hline
\end{tabular}

(a)

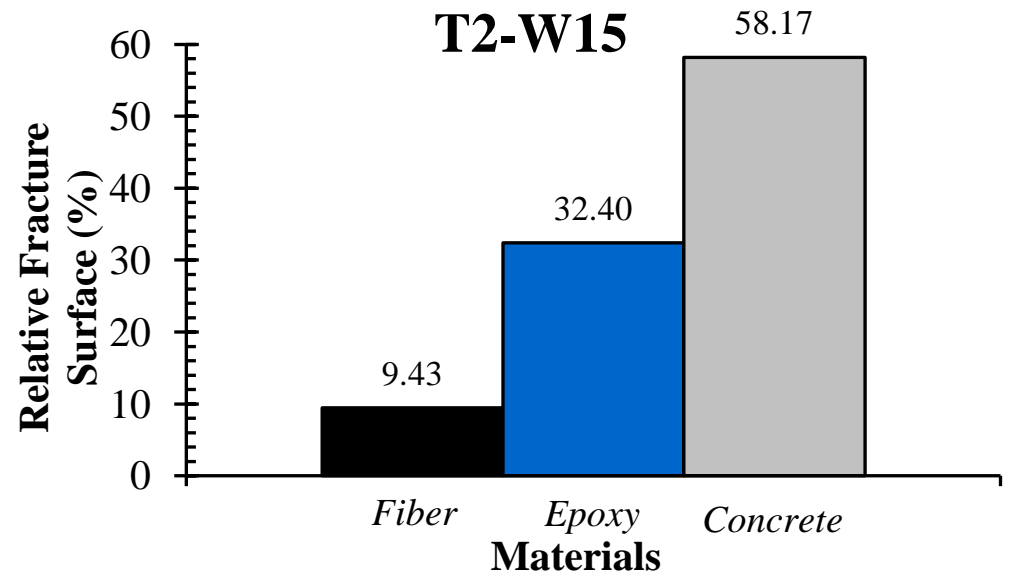

(b)

Figure 5-33 


\begin{tabular}{|c|c|c|c|}
\hline$D^{D}$ & $D$ & $D$ & $\triangleright$ \\
\hline${ }^{\triangleleft}$ Fibers: 0.71 & ${ }^{\triangleleft}$ Fibers: 0.83 & ${ }^{\triangleleft}$ Fibers: 1.64 & ${ }^{\triangleleft}$ Fibers: 0.41 \\
\hline Epoxy: $43 . \$ 0$ & Epoxy: 50.文6 & Epoxy: 53.63 & Epoxy: 35.02 \\
\hline Concrete: 55.99 & Concrete: 48.41 & Concrete: 44.73 & Concrete: 64.57 \\
\hline$\Delta$ & T3 & Wo11 & $\triangleleft \Delta$ \\
\hline
\end{tabular}

(a)

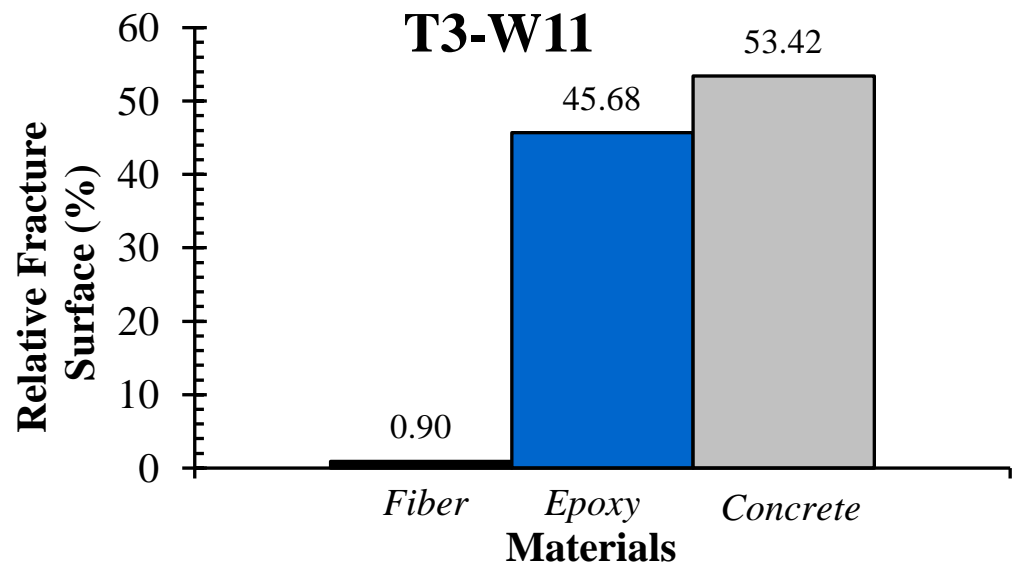

(b)

Figure 5-34

\begin{tabular}{|c|c|c|c|}
\hline$D$ & $D$ & $D$ & $D$ \\
\hline${ }^{\triangleleft}$ Fibers: 2.22 & ${ }^{\triangleleft}$ Fibers: 0.75 & ${ }^{\triangleleft}$ Fibers: 1.31 & ${ }^{\triangleleft}$ Fibers: 1.77 \\
\hline Eроху: 29.04 & Epoxy: $53 . \$ 6$ & Epoxy: 62.48 & Epoxy: 49.90 \\
\hline Concrete: 68.73 & Concrete: 45.69 & Concrete: 36.21 & Concrete: 48.33 \\
\hline$\Delta$ & T3. & W/13 & $\Delta$ \\
\hline
\end{tabular}

(a)

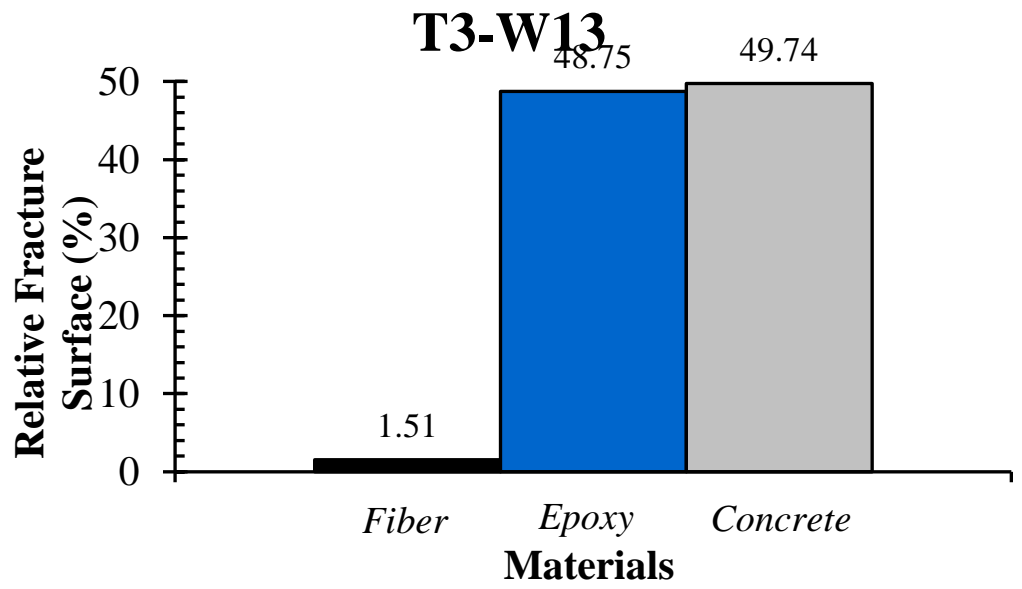

(b)

Figure 5-35 


\begin{tabular}{|c|c|c|c|}
\hline${ }_{D} D$ & $D^{D}$ & $D$ & $\Delta$ \\
\hline${ }^{\triangleleft}$ Fibers: 7.04 & ${ }^{\triangleleft}$ Fibers: 9.51 & Fibers: 13.78 & Fibers: 11.82 \\
\hline Epoxy: 32.68 & Epoxy: 29.\$2 & Epoxy: 44.\$8 & Epoxy: 35.94 \\
\hline Concrete: 60.28 & Concrete: 60.96 & Concrete: 41.84 & Concrete: 52.24 \\
\hline$\Delta$ & T3. & Wa15 & $\triangleleft \Delta$ \\
\hline
\end{tabular}

(a)

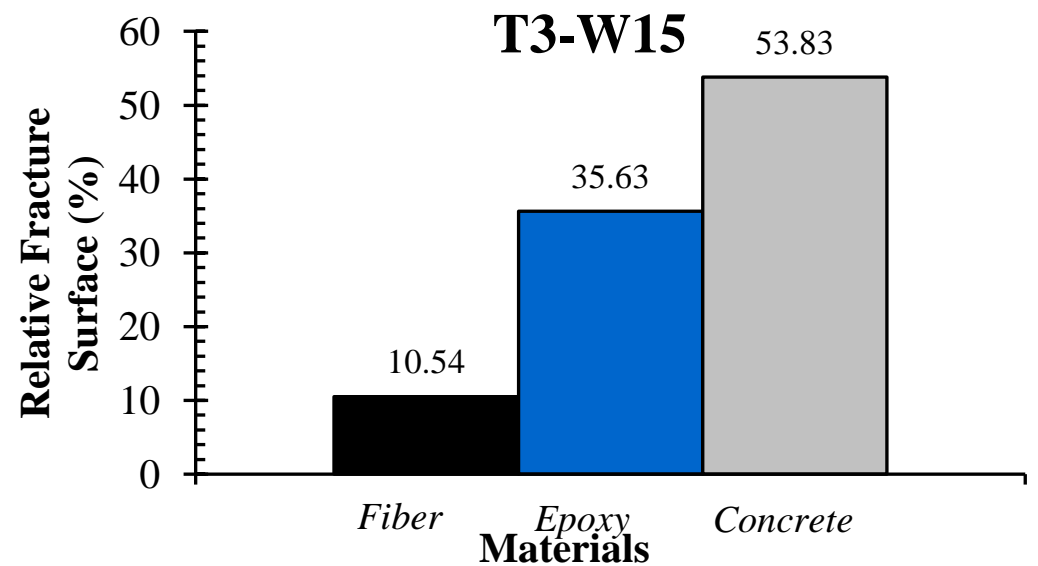

(b)

Figure 5-36

\begin{tabular}{|c|c|c|c|}
\hline$\nabla \quad \Delta^{D}$ & $D$ & $D$ & $\Delta$ \\
\hline${ }^{\triangleleft}$ Fibers: 0.67 & ${ }^{\triangleleft}$ Fibers: 1.72 & ${ }^{\triangleleft}$ Fibers: 1.18 & ${ }^{\triangleleft}$ Fibers: 7.73 \\
\hline Ероху: 0.8 & Epoxy: 97.Д8 & Epoxy: $94 . \$ 1$ & Epoxy: 71.ฎ3 \\
\hline Concrete: 98.46 & Concrete: 1.00 & Concrete: 4.21 & Concrete: 21.04 \\
\hline$\Delta$ & $T 4$ & $W_{k 11} \wedge$ & $\Delta$ \\
\hline
\end{tabular}

(a)

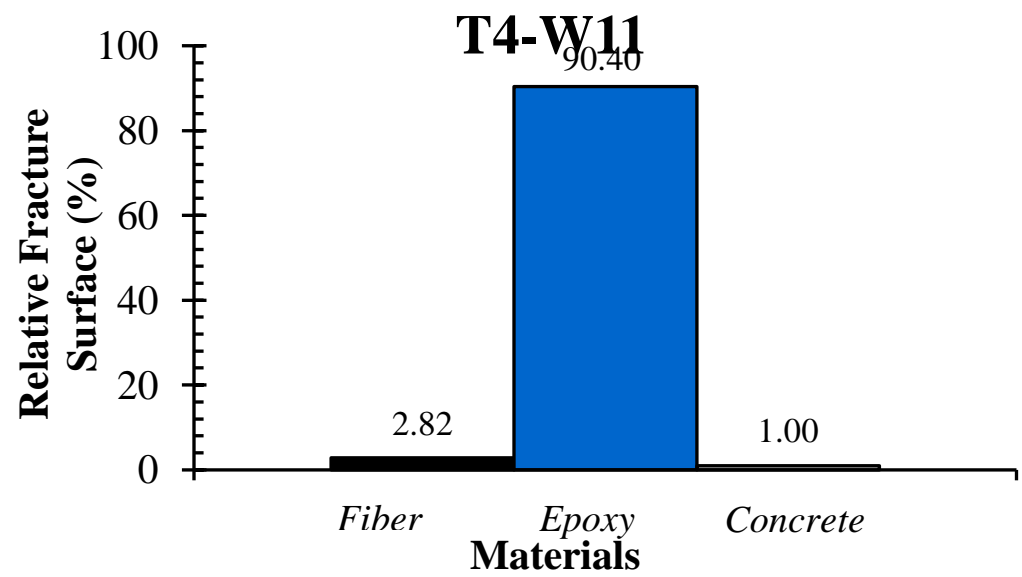

(b)

Figure 5-37 


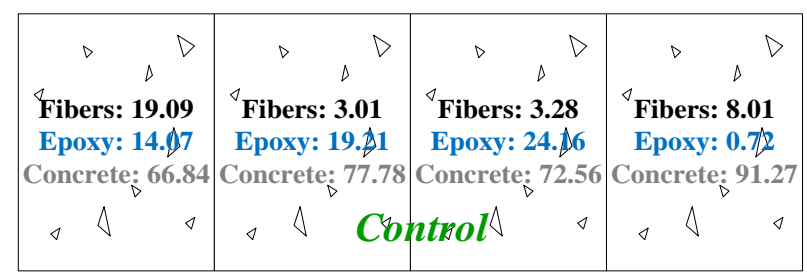

(a)

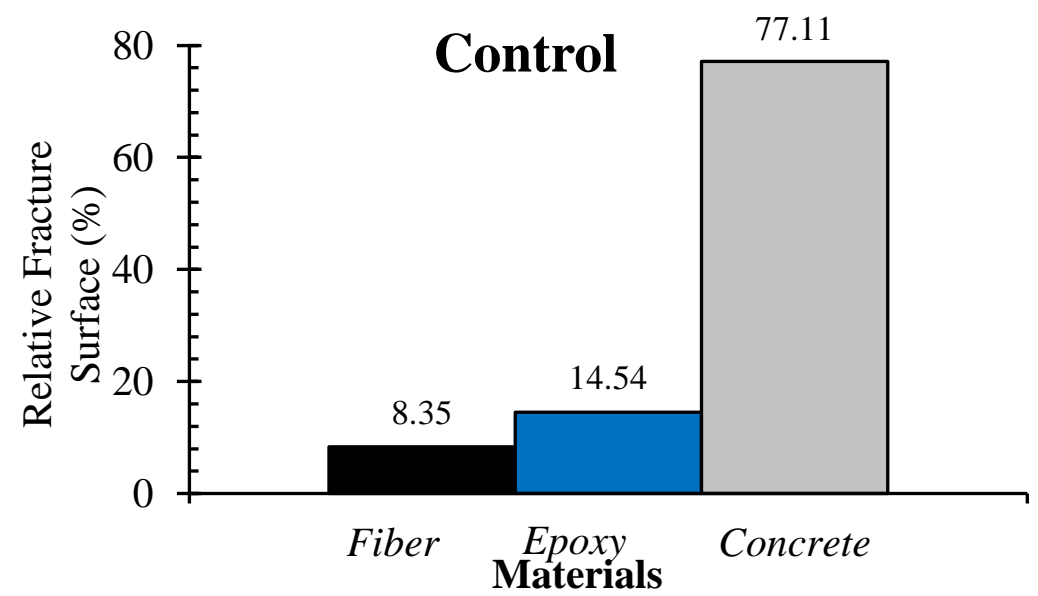

(b)

Figure 5-38

\section{$5.8 C M M$}

A 3-D surface profile of the FRP specimens investigated using optical microscopy was obtained using CMM. The coordinates of 240 points were recorded in a matrix by the CMM and analyzed in Origin 8.1 professional version. The area of the fractured surface was calculated, and the 3-D profile was plotted. Figure 5-39 and Figure 5-40 illustrate the percentile increase of the area for samples immersed in T1, T2, and T3 water for weeks 11, 13, and 15. A higher increase is an indication of cohesive failure, while a lower increase reveals a rather adhesive failure. Also, as the temperature and the immersion duration increases, less of a change in total area is observed, meaning the fracture tended to be more adhesive in nature. 


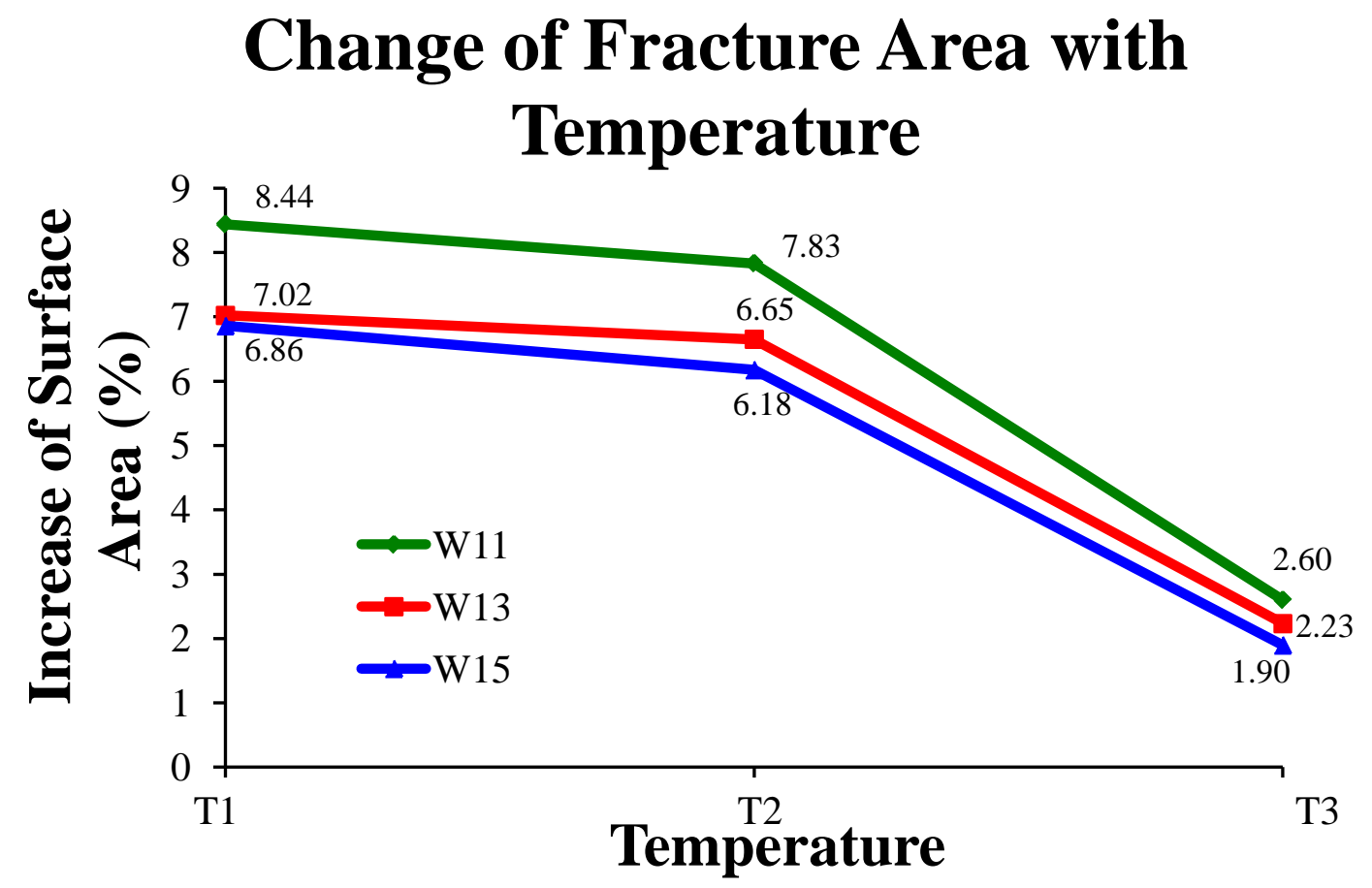

Figure 5-39 Change of Fracture Area with Temperature

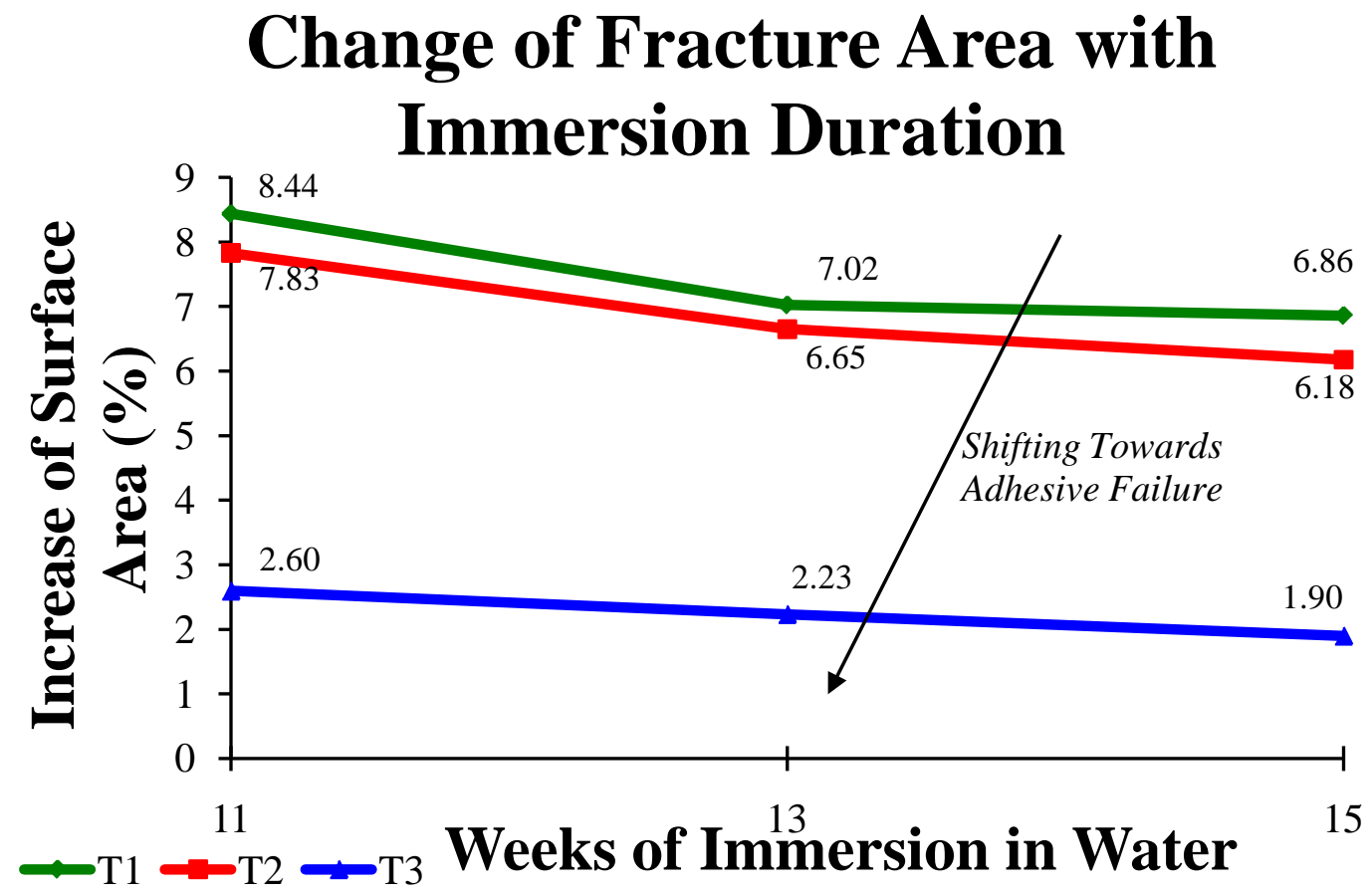

Figure 5-40 Change of Fracture Area with Immersion Duration

Specimens immersed in the T2 temperature tank were studied closely throughout the testing period. The surface area increase, as obtained by CMM, is illustrated in Figure 5-41. A clear and 
distinct shift toward the adhesive failure is noted, although between weeks 7 and 11, no sensible changes were observed.

\section{Change of Fracture Area with Immersion Duration (T2)}

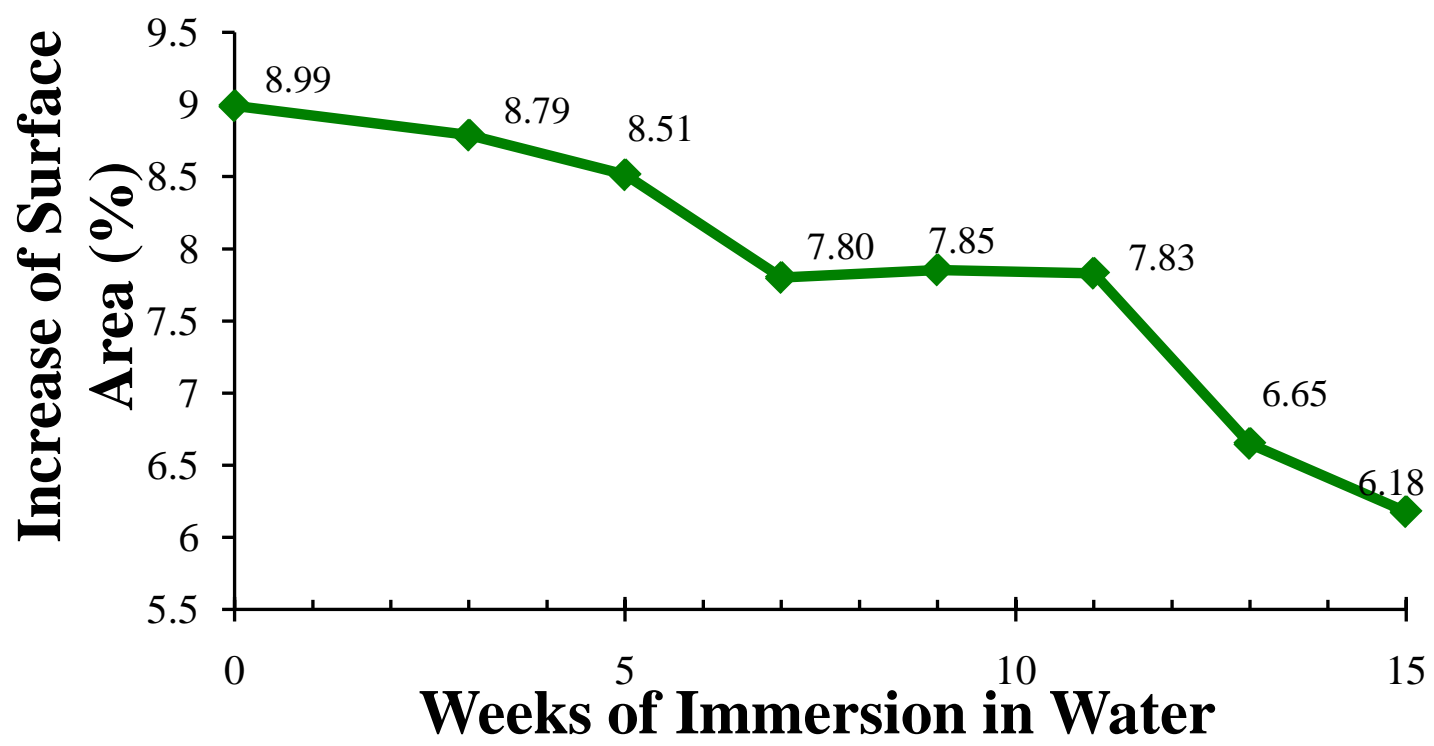

Figure 5-41 Change of Fracture Area with Immersion Duration (T2) 

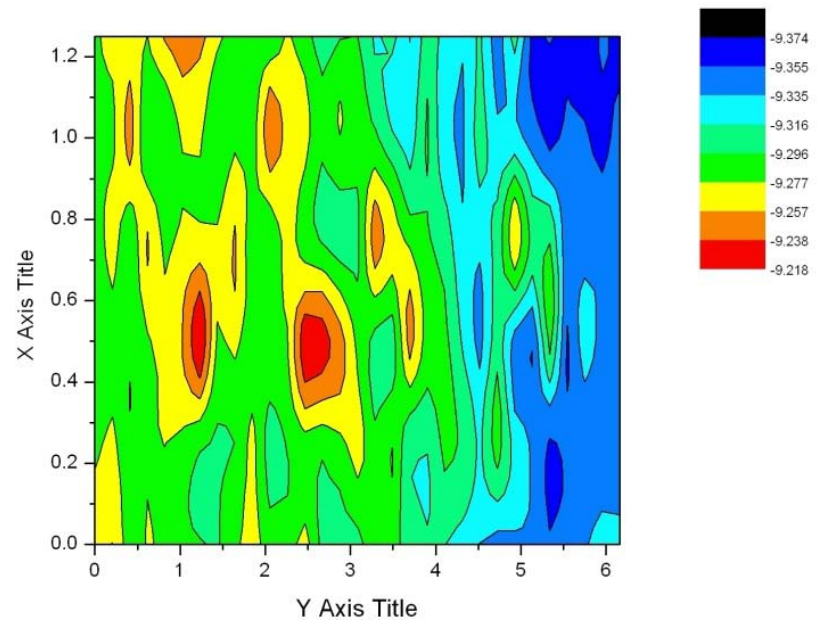

(a)
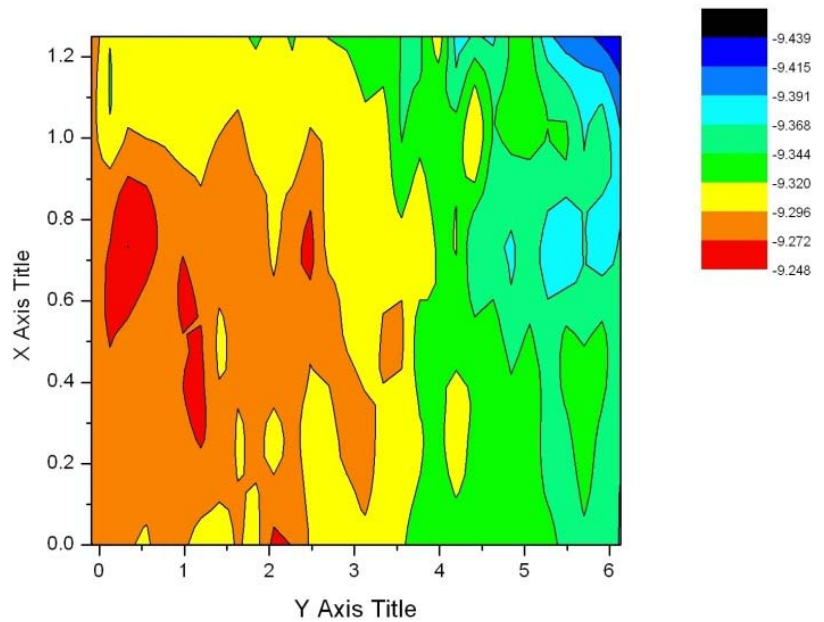

(b)
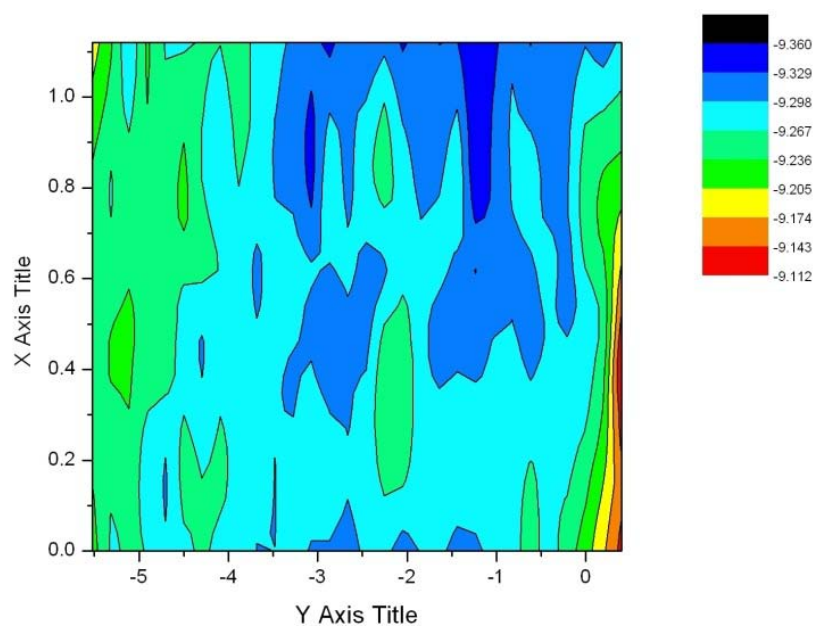

(c)

Figure 5-42 T1 Contours; (a) W11, (b) W13, (c) W15 


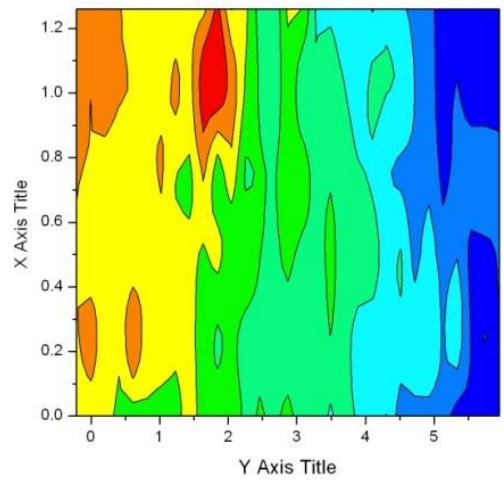

(a)

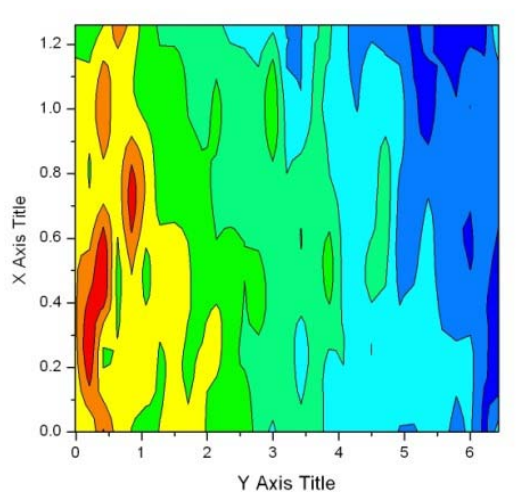

(b)

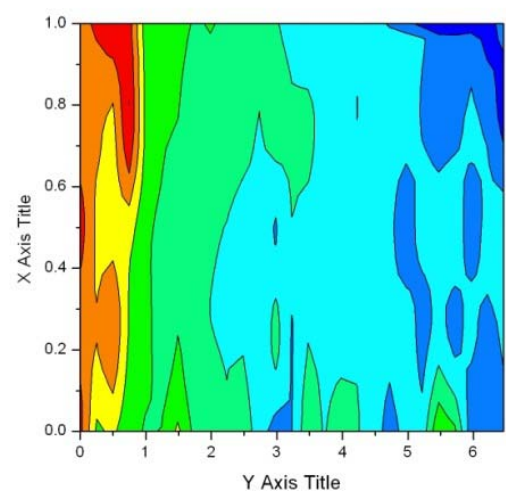

(c)

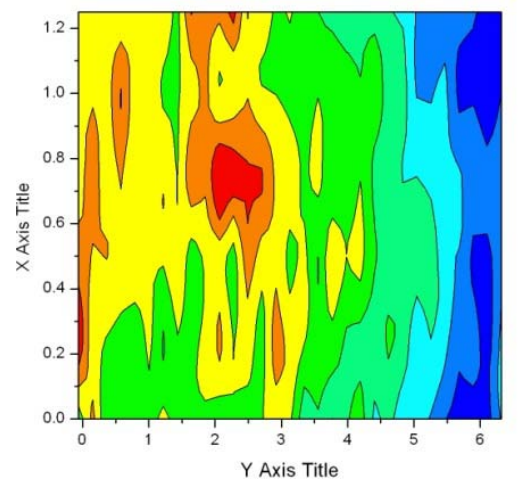

(d)

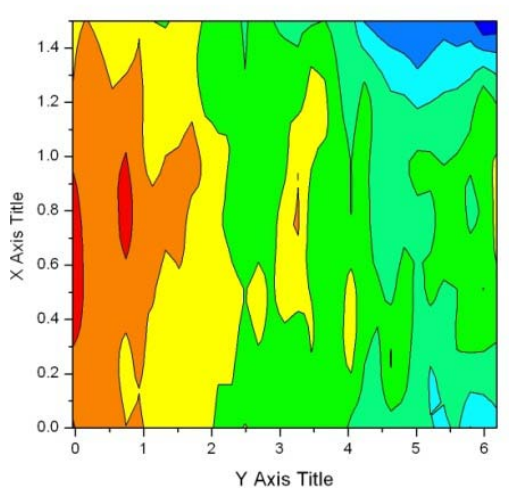

(e)
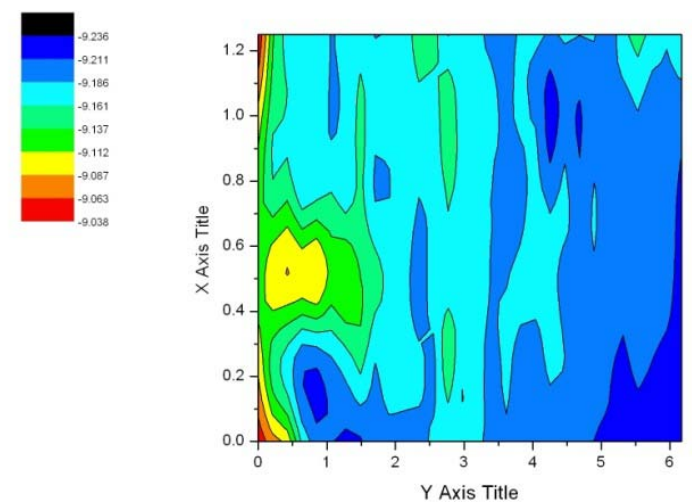

(f)

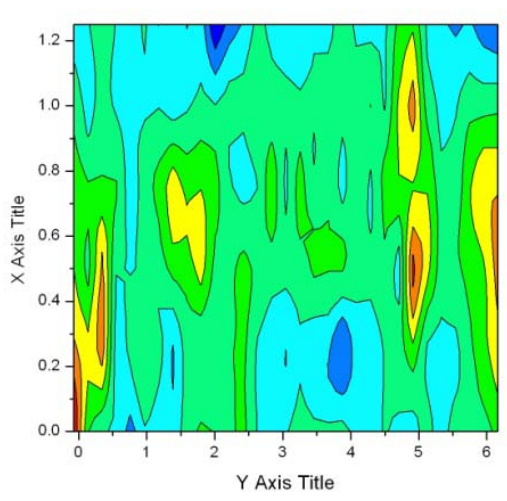

(g)
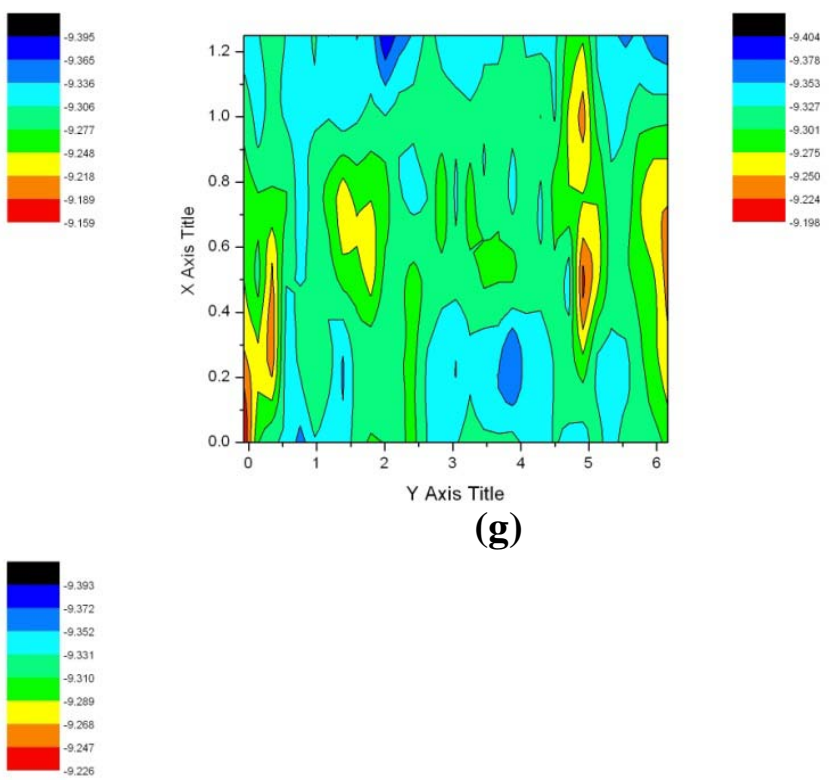

Figure 5-43 T2 Contours; (a) W3, (b) W5, (c) W7, (d) 9, (e) W11, (f) W13, (g) W15 

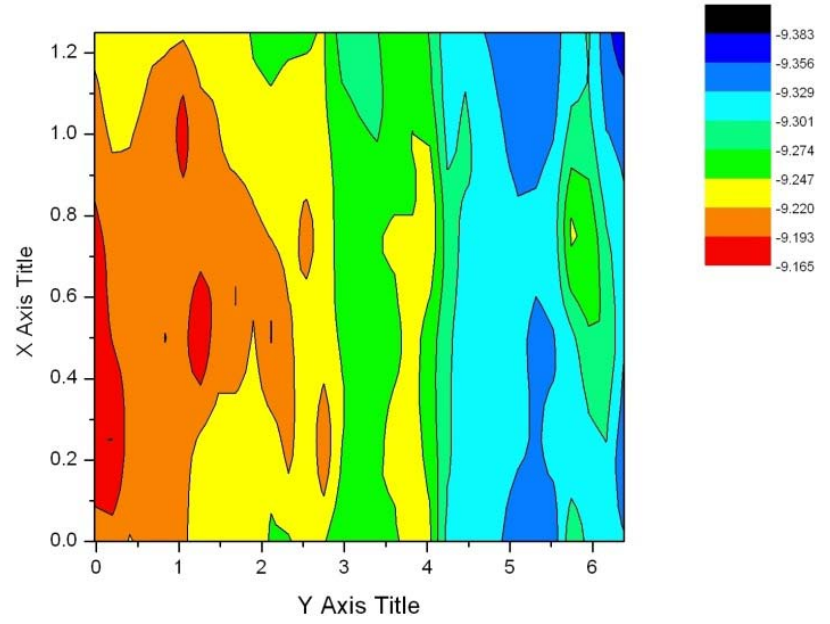

(a)
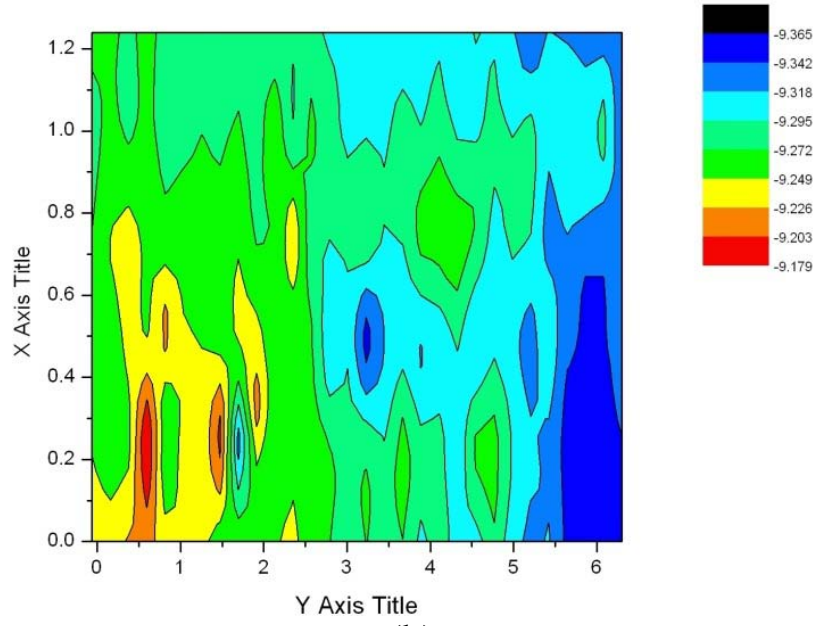

(b)
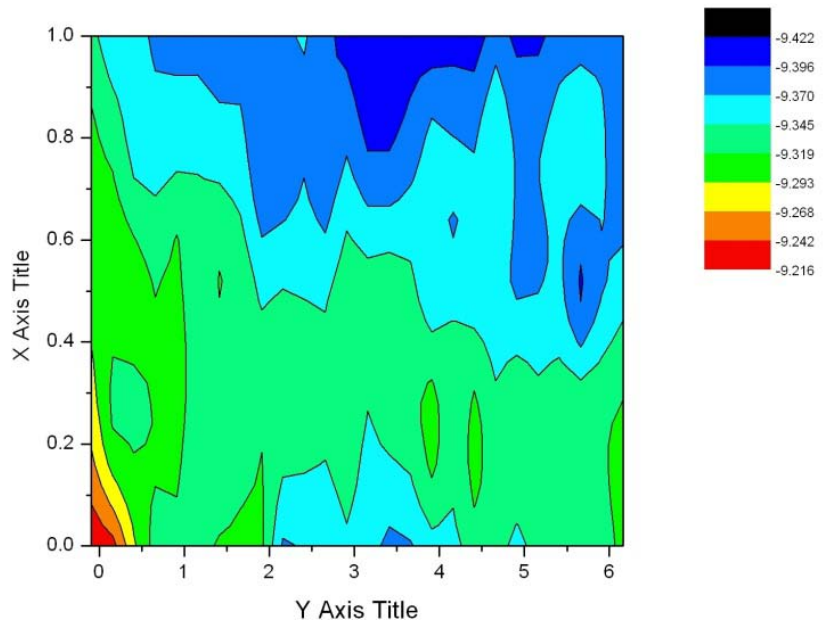

(c)

Figure 5-44 T3 Contours; (a) W11, (b) W13, (c) W15 
The contours also confirm that as the immersion period increases, the thickness of the concrete attached to the FRP decreases, thus indicating shift towards adhesive failure.

\subsection{Interface Relatively Humidity Measurement}

The Interface Relative Humidity (IRH) was measured using the Humitest System by James Instrument. The IRH increased from 57\% for control specimens to 79\% for T4-W15. Figure 5-45 shows that generally, the IRH increases with temperature and immersion duration. Consequently, it can be concluded that the presence of moisture at the interface can cause the failure shift from cohesiveness to adhesiveness. Except for the T3 specimens, IRH kept relatively constant between weeks 7 and 11 for the other temperature.

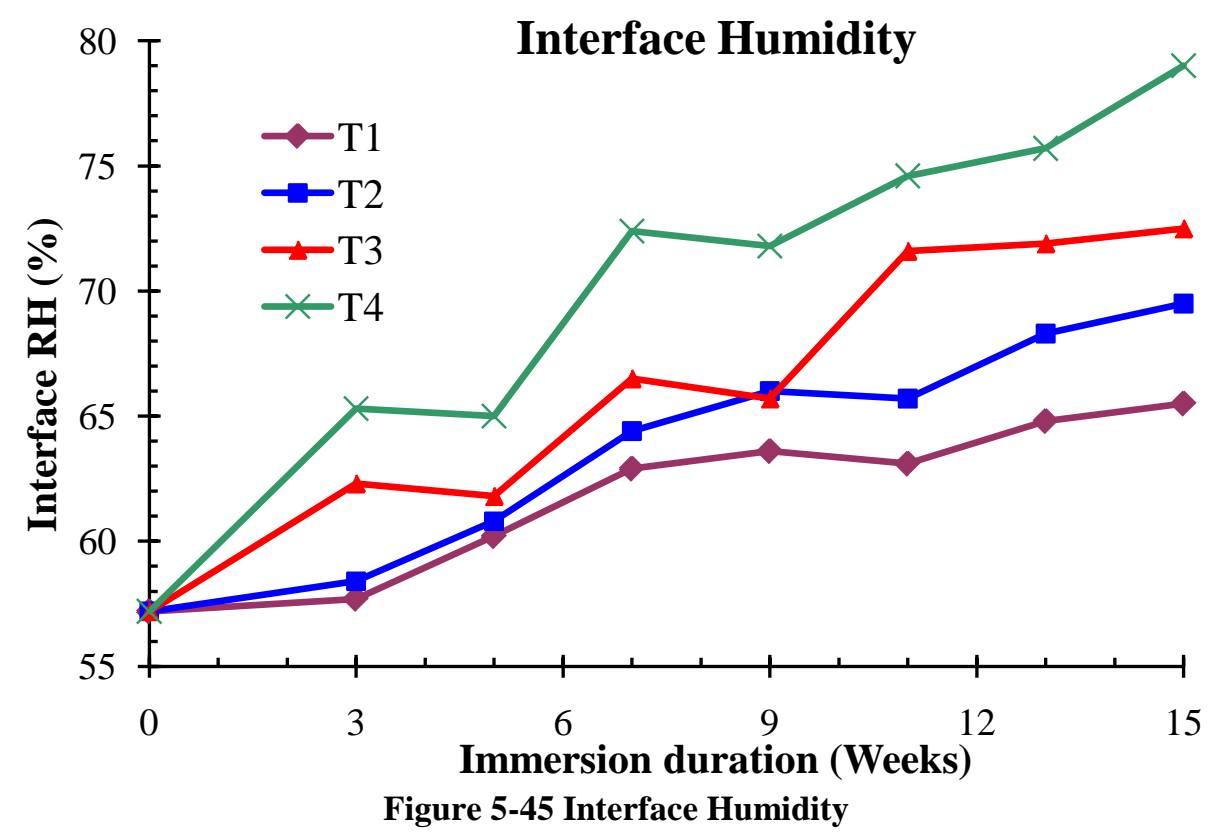

\subsection{Pull-Off Tests}

Pull-off tests were performed to obtain both FRP-concrete interface pull-off and concrete surface tensile strengths. The concrete pull-off strength increases, as opposed to the interface pull-off strength, which decreases with increasing immersion duration and temperature. This phenomenon may be partly attributed to the continuous cement hydration (See Figure 5-46 and Figure 5-47). 


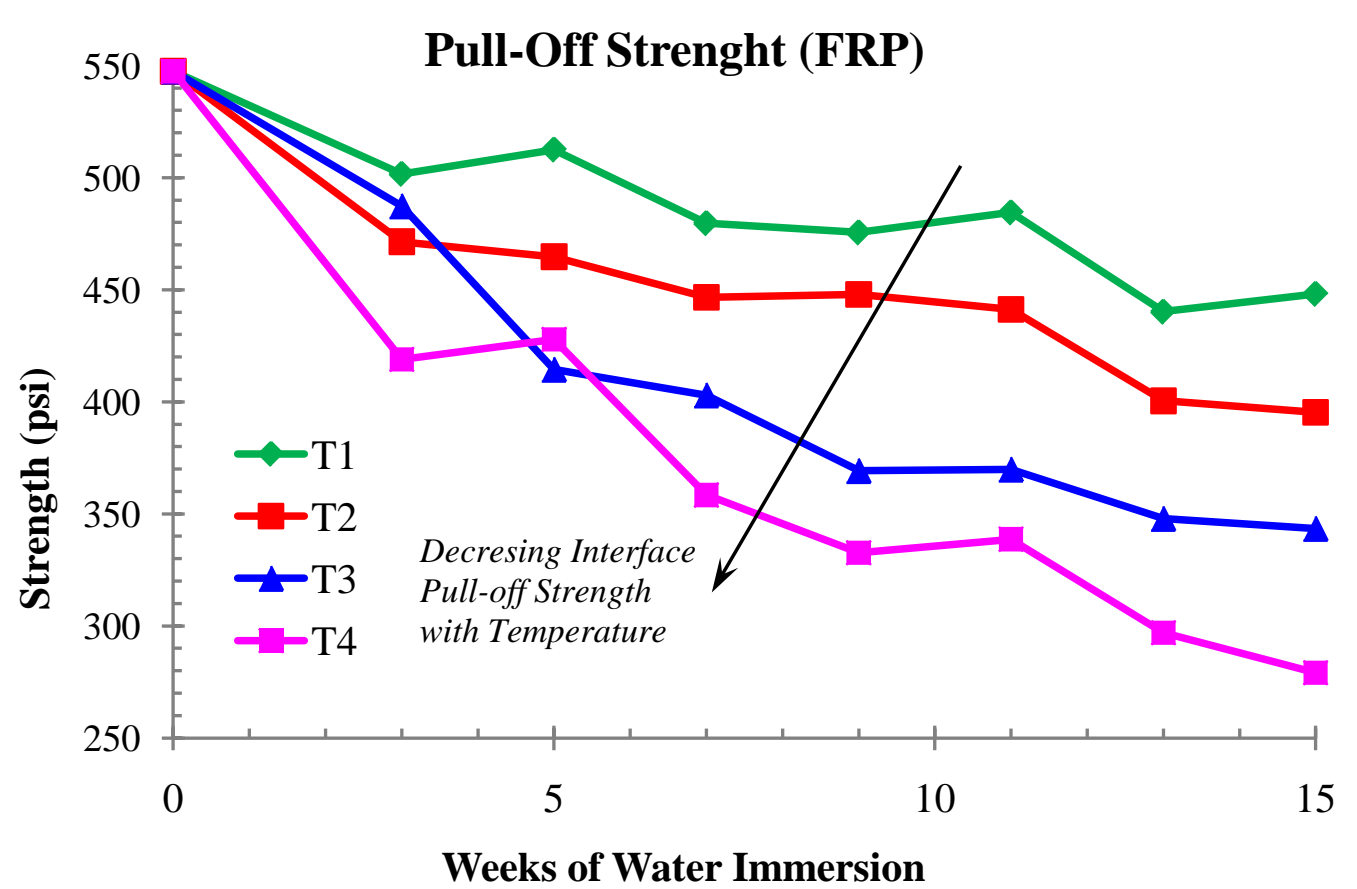

Figure 5-46 Interface Pull-off Strength

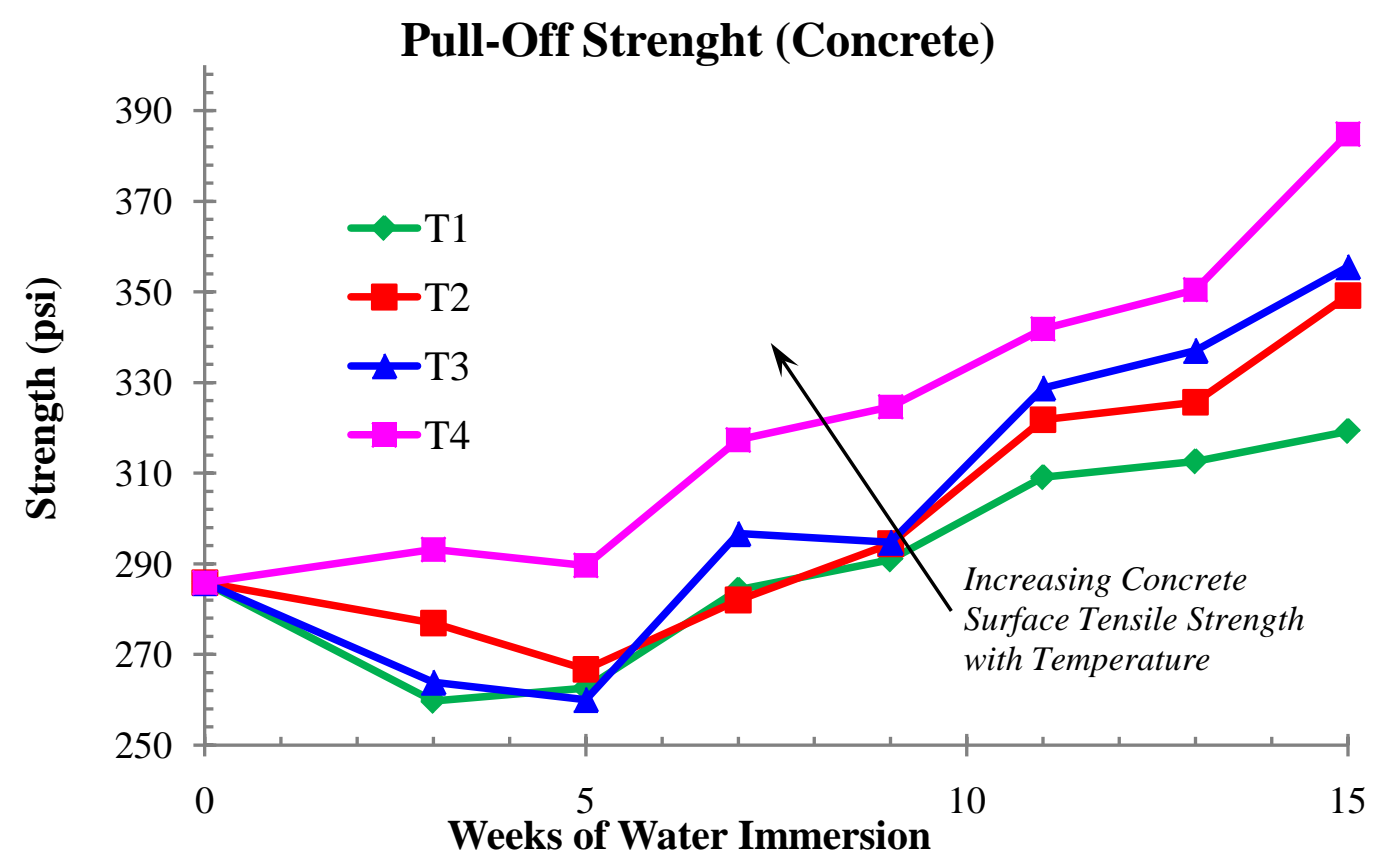

Figure 5-47 Concrete Surface Tensile Strength 


\subsection{Discussion}

Generally, in consideration of the totality of measured and/or calculated properties, which include ERR, pull-off strength, relative fractured surface, and IRH, a rather sharp drop is observed during the early stages of water immersion. The property then keeps relatively constant, or at least changes at a very low rate between the $7^{\text {th }}$ and $11^{\text {th }}$ weeks of immersion.

Among all other testing results, the pull-off strength and the ERR appear to have more of correlation, in comparison to the other properties mentioned above. Both values experience a sharp drop at the third week of immersion for all the temperatures. Between weeks 7 and 9, both the pull-off strength, and the ERR levels remain relatively constant, and finally another sharp drop is observed between weeks 11 and 13 of immersion. Both of these properties revealed a small jump from week 3 to week 5 for T4 specimens, which were followed by steady reduction for both. Overall, the general trend for both of these properties was almost the same for all the temperatures and immersion periods, which can lead to the conclusion that the failure load, and consequently the ERR is dependent on the pull-off strength.

The results of both optical microscopy and CMM depict a shift in the failure mode from cohesive toward adhesive failure.

As for the traction-separation law, although the $\tau$-s curves from the traditional and J-Integral methods are not the same and have different maximum shear stress and slip, the areas under the curves, which represent the ERR, are almost the same as calculated using both methods. The ERR calculated from Equation (5-1), which only uses the maximum load as the experimental value, is also close to those obtained by calculating the area under the $\tau$-s curves. Therefore, it appears that the use of ERR as the failure criterion as opposed to maximum shear stress or maximum strain level is more reasonable.

A correlation Analysis was performed on the ERR and three other test parameters, .e., pulloff strength, concrete surface tensile strength, and the IRH. The crosscorr MATLAB function with a 95\% confidence interval using a polynomial function was utilized. Figure 5-48 shows the results for the Pull-off strength vs. ERR for each temperature. The corresponding $\mathrm{R}^{2}$ values are shown in Table 5-9. A very good correlation is observed for all the temperatures with an average $\mathrm{R}^{2}$ of 0.876 . The results of a similar analysis between ERR and CSTS, as well as ERR and IRH are depicted in Figure 5-49 and Figure 5-50, respectively. Despite the average $\mathrm{R}^{2}$ of 0.722 for CSTS, T1 and T2 do not exhibit a strong correlation ( $\mathrm{R}^{2}$ about 0.63$)$. IRH however reveals a 
rather good correlation with average $\mathrm{R}^{2}$ of 0.8711 . Generally, the best correlation (highest $\mathrm{R}^{2}$ ) was observed for the parameters obtained at the highest temperature, T4.

It can be concluded that ERR makes a reliable choice of failure criterion for FRP-concrete interface since all the other parameter exhibit more or less good correlation with ERR.

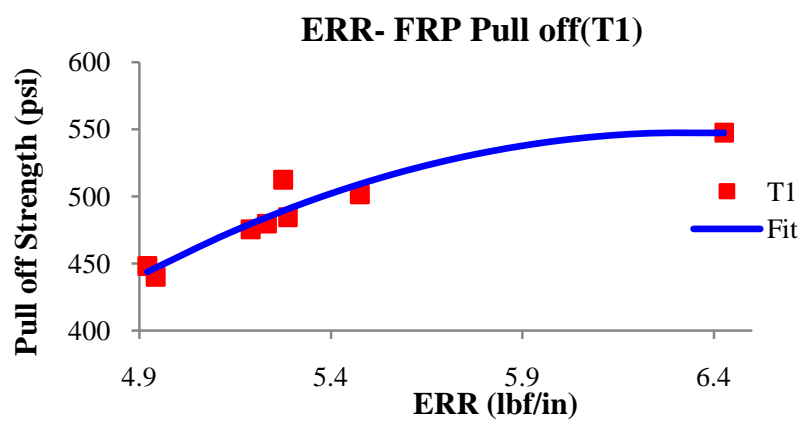



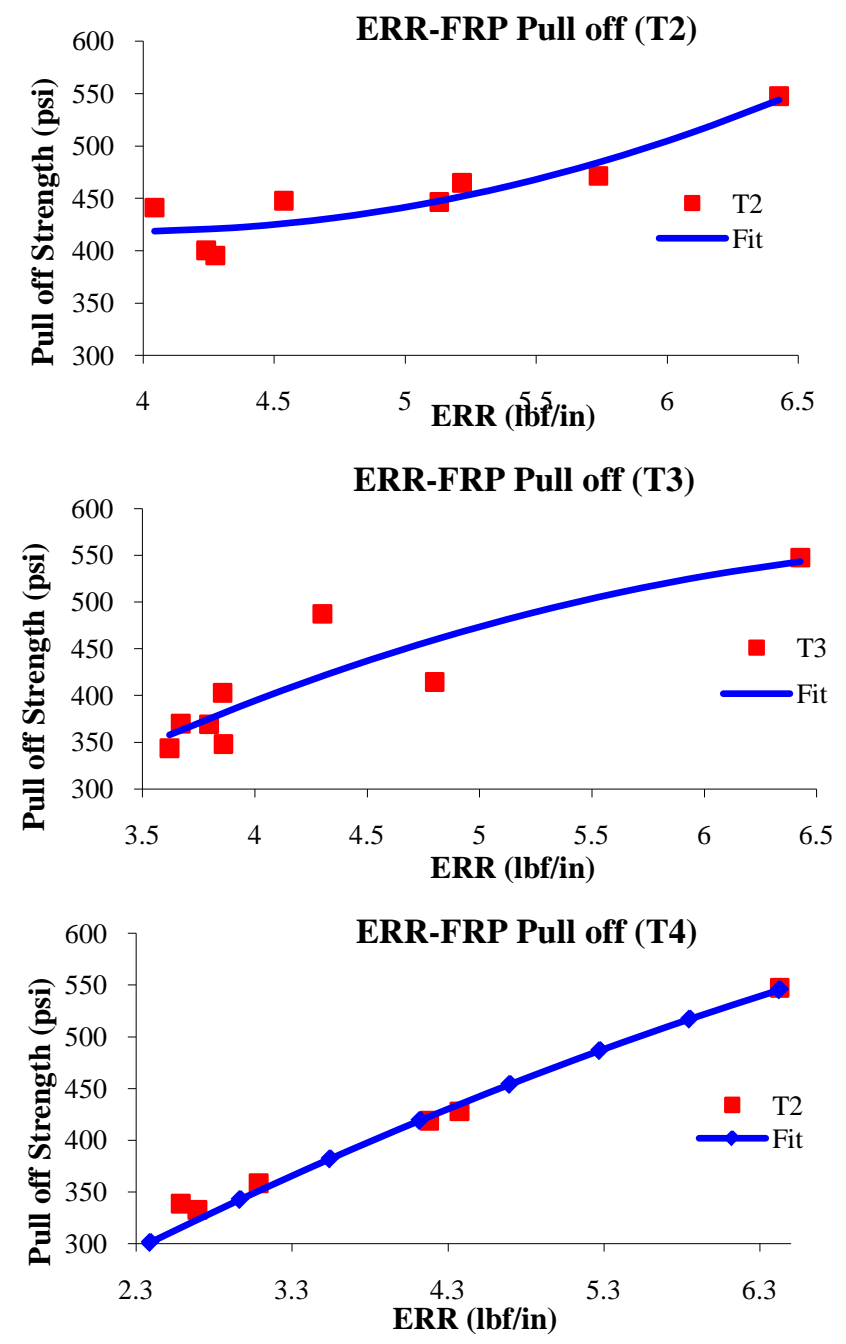

Figure 5-48 ERR- FRP Pull off Strength

Table 5-9 ERR- FRP Pull off Strength

\begin{tabular}{ccccc}
\hline \multicolumn{5}{c}{ ERR- FRP Pull off } \\
\hline & T1 & T2 & T3 & T4 \\
$\mathbf{R}^{2}$ & 0.9123 & 0.8482 & 0.7702 & 0.9746 \\
\hline
\end{tabular}

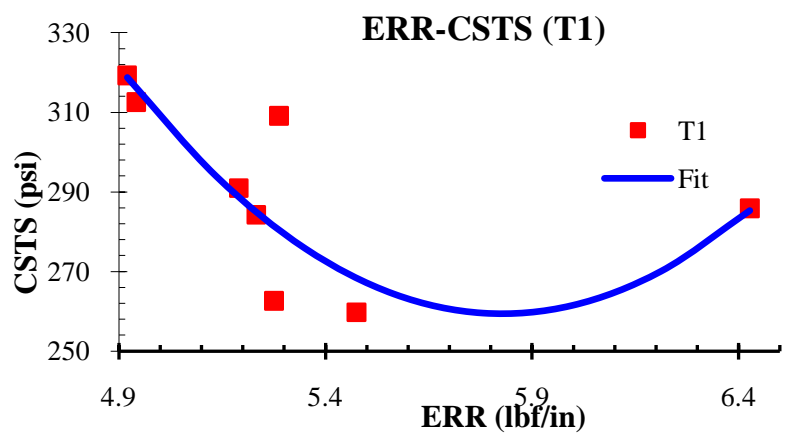



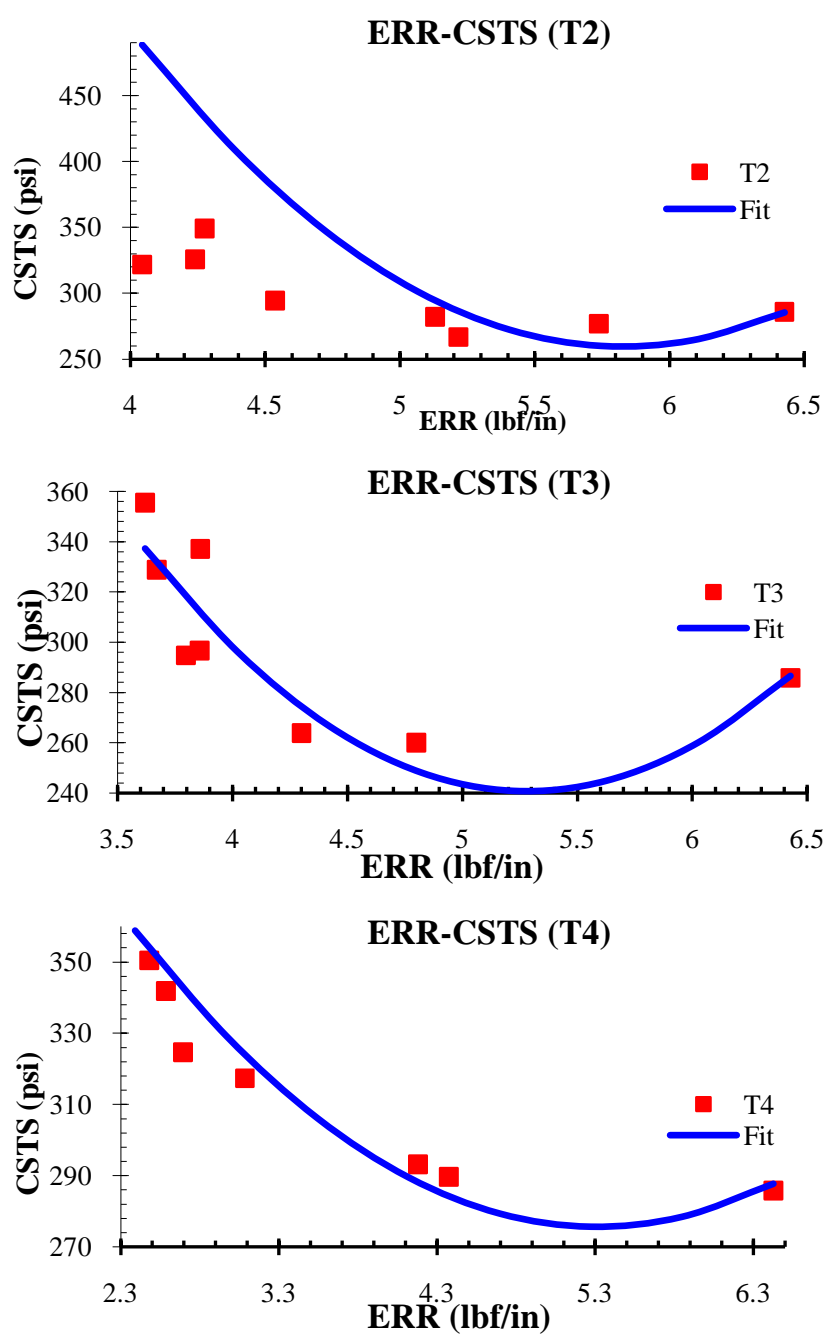

Figure 5-49 ERR-Concrete Surface Tensile Strength

Table 5-10 ERR-Concrete Surface Tensile Strength

\begin{tabular}{ccccc}
\hline \multicolumn{5}{c}{ ERR-CSTS } \\
\hline & T1 & T2 & T3 & T4 \\
\hline $\mathbf{R}^{2}$ & 0.6325 & 0.6325 & 0.7612 & 0.863 \\
\hline
\end{tabular}



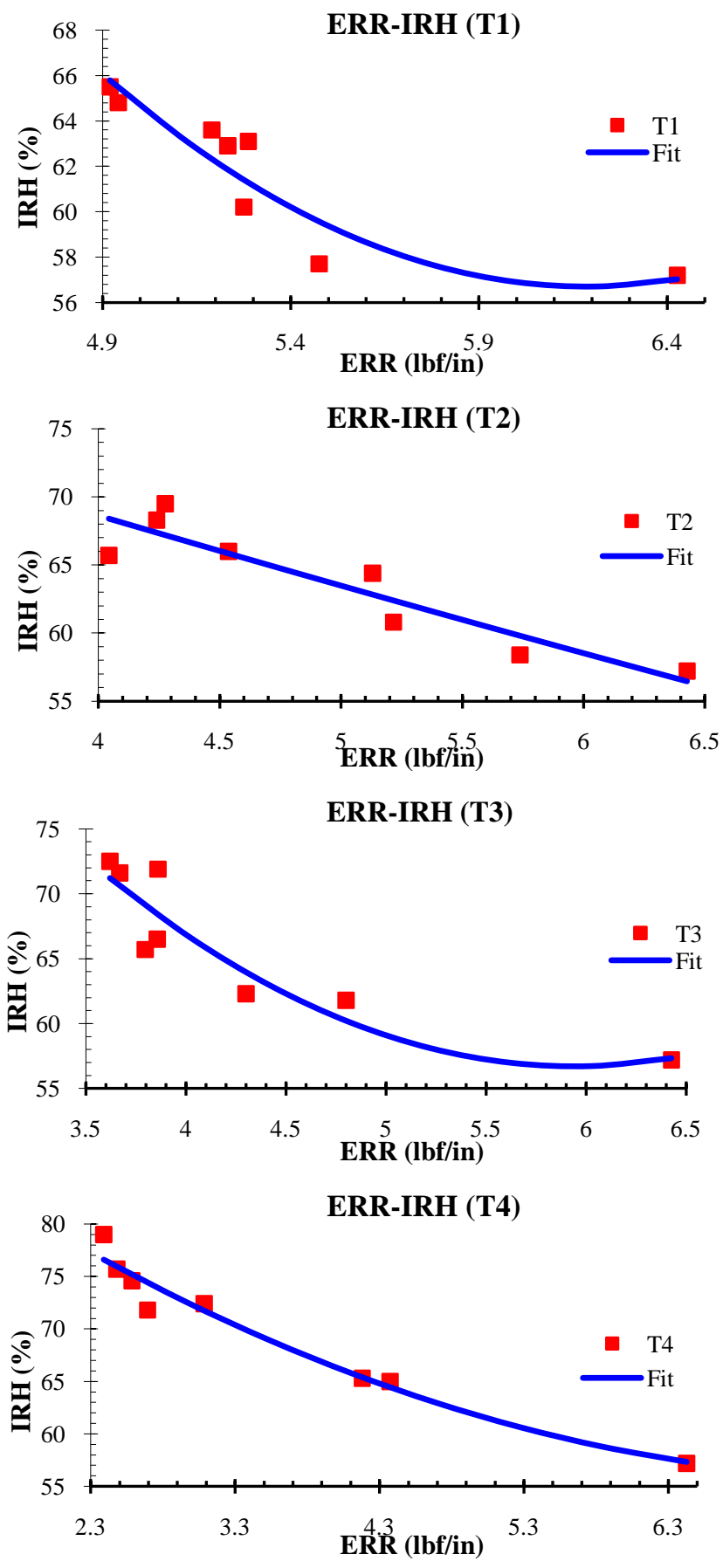

Figure 5-50 ERR-IRH

Table 5-11 ERR-IRH

\begin{tabular}{ccccc}
\hline \multicolumn{5}{c}{ ERR-IRH } \\
\hline & $\mathbf{T 1}$ & $\mathbf{T} 2$ & $\mathbf{T 3}$ & $\mathbf{T 4}$ \\
$\mathbf{R}^{2}$ & 0.8336 & 0.8533 & 0.8364 & 0.9614 \\
\hline
\end{tabular}




\section{Concluding Remarks and Recommendations}


Fiber reinforced polymer (FRP) composites have been used for reinforcement of conventional materials in automobile, marine, and aerospace engineering for decades. Rehabilitation and retrofit of civil infrastructures, especially bridges, have been gained more popularity both in research and industry level. The significant need to rehabilitate and retrofit the deficient bridges worldwide has created a great opportunity to use FRP technology in the form of externally bonded plates or sheets to meet this need which necessitate acquiring a more sound understanding of the FRP-concrete interface characteristics.

In this study, the interface durability of concrete with externally bonded FRP laminate was studied under Mode-II loading condition using single shear testing setup. Accelerated aging was achieved by exposing the specimens to water immersion combined with elevated temperature. The following conclusions can be drawn based on this study:

- The ERR decreases with respect to the age of the specimen.

- The elevated temperature has a more significant effect on interface degradation than the moisture effect. There is a threshold temperature beyond which the degradation mechanism of the interface significantly changes. This temperature must be related to the glass transition temperature of the epoxy, and may adversely suppress using this technology in regions with average annual temperature above $110^{\circ} \mathrm{F}$.

- The proposed path-independent J-integral is an effective method for evaluating the interfacial behavior, which only requires the measurement of the relative slip at the crack tip and the corresponding load, whereby the effort of bonding strain gages for the traditional method can be omitted. It can accurately describe the facture energy; however, the traction-separation law seems to be affected by the accuracy of the relative slip.

- The failure modes for most of the specimens were cohesive at the early age. As the exposure duration and the temperature increased, the failure mode shifted from cohesive to adhesive, with less concrete attached to the FRP laminates. This visual observation was also confirmed with the results of the optical microscopy and CMM. 


\subsection{Recommendations}

Since application of FRP materials in civil infrastructures is a fairly new technology, there are a variety of parameters which can be further studied both on the macro and micro level of the FRP-concrete interface.

In this study, the materials in use included normal concrete, CFRP, and one type of epoxy resin only. High performance concrete, as well as different types of FRP, e.g., glass FRP (GFRP), and different types of resins can be used.

This research was limited to pure Mode II fracture, which can be further extended to mixed mode loading by designing an appropriate testing fixture. Cyclic loading can also be studied to investigate the fatigue characteristics of the interface.

The environmental conditioning parameters in this research were only limited to elevated temperatures and water immersion which can be further extended to more sever realistic conditions such as freezing and thawing, wetting and drying, acidic attack, sulfate attack, and so forth.

Finite element method can be used to numerically verify the results of this experimental study. 


\section{References}


1. Boyajian, D.M., Mode I fracture and durability of the CFRP-concrete interface bond, in Civil and Environmental Engineering. 2002, West Virginia University: Morgantown, WV. p. 432.

2. Subramaniam, K.V., C.C. b, and L. Nobile, Width effect in the interface fracture during shear debonding of FRP sheets from concrete. Engineering Fracture Mechanics, 2007 a. 74: p. 578-594.

3. Suo, Z. and J.W. Hutchinson, Interface Crack between Two Elastic Layers. International Journal of Fracture, 1990. 43: p. 1-18.

4. Yuan, H., et al., Full-range behavior of FRP-to-concrete bonded joints. Engineering Structures, 2004. 26: p. 553-565.

5. Qiao, P., J. Wang, and J.F. Davalos, Analysis of tapered ENF specimen and characterization of bonded interface fracture under Mode-II loading. Solids and Structures, 2003. 40: p. 1865-1884.

6. Ferracuti, B., Strengthening of RC structures by FRP: Experimental Analysis and Numerical Modeling, in Mechanical. 2006, University of Bologna: Bologna. p. 288.

7. Högberg, J.L., Mixed mode cohesive law. International Journal of Fracture, 2006. 141: p. 549-559.

8. Nakaba, K., et al., Bond Behavior between Fiber-Reinforced Polymer Laminates and Concrete. ACI Structural Journal, 2001. 98(3): p. 359-367.

9. Wang, J., Debonding of FRP-plated reinforced concrete beam, a bond-slip analysis. I. Theoretical formulation. International Journal of Solids and Structures, 2006. 43: p. 6649-6664.

10. Wang, J., Cohesive zone model of FRP-concrete interface debonding under mixed-mode loading. International Journal of Solids and Structures, 2007 b. 44: p. 6551-6568.

11. Xu, X.P. and A. Needleman, Void nucleation by inclusion debonding in a crystal maatrix. Modeling and Simulation in Materials Science and Engineering, 1993. 1(2): p. 111-132.

12. Anderson, T.L., Fracture Mechanics; Fundamentals and Applications. 3 ed. 2005: CRC.

13. Rice, J.R., A Path Independent Integral and the Approximate Analysis of Strain Concentration by Notches and Cracks. Journal of Applied Mechanics, 1968. 35: p. 379-386. 
14. Oritz, M. and A. Pandolfi, Finite-Deformation Irreversible Cohesive Element for Three-Dimensional Crack-Propagation Analysis. International Journal of Numerical Methods in Engineering, 1999. 44: p. 1267-1282.

15. Needleman, A., A Continuum Model for Void Nucleation by Inclusion Debonding. Journal of Applied Mechanics, 1987. 54: p. 525-531.

16. Hillerborg, M., M. Modeer, and P. Peterson, Analysis of crack formation and crack growth in concrete by means of fracture mechanics and finite elements. Cement and Concrete Research, 1976. 6: p. 773-782.

17. Camanho, P.P. and C.G. Dávila, Mixed-Mode Decohesion Finite Elements for the Simulation of Delamination in Composite Materials. 2002, NASA: Hampton, VA.

18. Elices, M., et al., The cohesive zone model: advantages, limitations and challenges. Engineering fracture mechanics, 2002. 69(2): p. 137-163.

19. Qiao, P. and Y. Chen, Cohesive fracture simulation and failure modes of FRPconcrete bonded interfaces. Theoretical and Applied Fracture Mechanics, 2008. 49: p. 213-225.

20. Bosch, M.J.v.d., P.J.G. Schreurs, and M.G.D. Geers, An improved description of the exponential Xu and Needleman cohesive zone law for mixed-mode decohesion. Engineering Fracture Mechanics, 2006. 73: p. 220-1234.

21. Bazant, Z.P., I.M. Daniel, and Z. Li, Size Effect and Fracture Characteristics of Composite Laminates. Journal of Engineering Materials and Technology, 1996. 118: p. 317-324.

22. Taljsten, B., "Strengthening of concrete prisms using the platebonding technique. International Journal of Fracture, 1996. 82: p. 253-266.

23. Barenblatt, G.I., Mathematical Theory of Equilibrium Cracks in Brittle Failure. Advances in Applied Mechanics, 1962. 7.

24. Ali-Ahmad, M., K.V. Subramaniam, and M. Ghosn, Debonding of FRP from Concrete in Strengthening Applications: Experimental Investigation and Theoretical Validation, in Civil Engineering. 2005, The City University of New York: New York. p. 177.

25. Dai, J., T. Ueda, and Y. Sato, Development of the Nonlinear Bond Stress-Slip Model of Fiber Reinforced Plastics Sheet-Concrete Interfaces with a Simple Method. Composites for Construction, 2005. 9(1): p. 52-62. 
26. Mazzotti, C., B. Ferracuti, and M. Savoia. An experimental study on FRP concrete delamination. in Fracture Mechanics of Concrete and Concrete Structures. 2004. Colorado, U.S.A.

27. Wang, J., Cohesive-bridging zone model of FRP-concrete interface debonding. Engineering Fracture Mechanics, 2007 a. 74: p. 2643-2658.

28. Wu, Z., H. Yuan, and H. Niu, Stress transfer and fracture propagation in different kinds of adhesive joints. Engineering Mechanics, ASCE, 2002. 128(5): p. 562-573.

29. Yuan, H., Z. Wu, and H. Yoshizawa, Theoretical solutions on interfacial stress of externally bonded steel/composite laminates. Structural Engineering/Earthquake Engineering, 2001. 18(1): p. 27-39.

30. Bizindavyi, L. and K.W. Neale, TRANSFER LENGTHS AND BOND STRENGTHS FOR COMPOSITES BONDED TO CONCRETE. Journal of Composites for Construction, 1999. 3(4): p. 153-160.

31. Chajes, M.J., et al., Bond and force transfer of composites materials plates bonded to concrete. ACI Structural Journal, 1996. 93: p. 209-217.

32. Yao, J., J.G. Teng, and J.F. Chen, Experimental study on FRP-to-concrete bonded joints. Composite: Part B 2005. 36: p. 99-113.

33. Karbhari, V.M., Durability of FRP Composites for Civil Infrastructure - Myth, Mystery or Reality. Advances in Structural Engineering, 2003. 6(3): p. 243-255.

34. Karbhari, V.M. and L. Zhao, Issues related to composite plating and environmental exposure effects on composite-concrete interface in external strengthening. Composite structures, 1998. 40(3-4): p. 293-304.

35. Chen, J.F. and J.G. Teng, ANCHORAGE STRENGTH MODELS FOR FRP AND STEEL PLATES BONDED TO CONCRETE. JOURNAL OF STRUCTURAL ENGINEERING, 2001. 127(7): p. 784-791.

36. Lorenzis, L.D., B. Miller, and A. Nanni, Bond of fiber-reinforced polymer laminates to concrete. ACI Material Journal, 2001. 98(3): p. 256-264.

37. Maeda, T., et al. A Study on Bond Mechanism of Carbon Fiber Sheet. in Third International Symposium on Non-Metallic (FRP) 
Reinforcement for Concrete Structures. 1997. Japan: Japan Concrete Institute.

38. Taljsten, B., "Defining anchor lengths of steel and CFRP plates bonded to concrete. International Journal of Adhesion and Adhesives, 1997. 17(4): p. 319-327.

39. Yoshizawa, H., et al. Effect of sheet bonding condition on concrete members having externally bonded carbon fiber sheet. in Materials for the new millennium 1996. Washington, D.C., USA.

40. Ali-Ahmad, M.K., K.V. Subramaniam, and M. Ghosn, Analysis of Scaling and Instability in FRP-Concrete Shear Debonding for Beam-Strengthening Applications. Engineering Mechanics, ASCE, 2007. 133(1): p. 58-65.

41. Mazzotti, C., M. Sovia, and B. Ferracuti, An experimental study on delamination of FRP Plates bonded to concrete. Construction and Building Materials, 2008. 22: p. 1409-1421.

42. Kamel, A.S., A.E. Elwi, and R.J.J. Cheng, Experimental study on the behavior of carbon fiber reinforced polymer sheets bonded to concrete. Canadian Journal of Civil Engineering, 2006. 33: p. 1438-1449.

43. Ali-Ahmad, M., K. Subramaniam, and M. Ghosn, Experimental Investigation and Fracture Analysis of Debonding between Concrete and FRP Sheets. Journal of Engineering Mechanics, 2006. 132(9): p. 914-923.

44. Pan, J. and C.K.Y. Leung, Effect of Concrete Composition on FRP/Concrete Bond Capacity. Journal of Composites for Construction, 2007 a. 11(6): p. 611-618.

45. Popovics, S., Numerical Approach to the Complete Stress-Strain Curve of Concrete. Cement and Concrete Research, 1973. 3(5): p. 583-599.

46. Lu, X.Z., et al., Bond-slip models for FRP sheets/plates bonded to concrete. Engineering Structures, 2005. 27: p. 920-937.

47. Pham, H.B. and R. Al-Mahaidi, Modelling of CFRP-concrete shear-lap tests Construction and Building Materials, 2006. 21(4): p. 727-735

48. Coronadoa, C.A. and M.M. Lopezb, Sensitivity analysis of reinforced concrete beams strengthened with FRP laminates Cement and Concrete Composites, 2005. 28(1): p. 102114. 
49. Teng, J.G., J.W. Zhang, and S.T. Smith, Interfacial stresses in reinforced concrete beams bonded with a soffit plate: A finite-element study. Construction and Building Materials, 2002. 16(1): p. 1-14.

50. Wang, W.Q. and J.F. Davalos, Modeling RC Beams Strengthened with Steel or FRP Plates, in 4th International Conference on Advanced Composite Materials in Bridges and Structures. 2004 Calgary, Alberta.

51. Anil, O. and C.M. Belgin, Review of Bond-Strength Models and Application on CFRP-to-Concrete Bonded Joints across Crack. science and engineering of composite materials, 2008. 15(2): p. 141-158.

52. Cousins, T.E., J.J. Lesko, and B. Carlin. Tailored Performance and Durability of Reinforced Concrete Beams Strengthened with FRP Plates. in Second International Conference on Composites in Infrastructure (ICCI). 1998.

53. Karbhari, V.M., et al., Durability Gap Analysis for Fiber-Reinforced Polymer Composites in Civil Infrastructure. Composites for Construction, 2003. 7(3): p. 238-247.

54. Chen, Y., J.F. Davalos, and I. Ray, Durability Prediction for GFRP Reinforcing Bars Using Short-Term Data of Accelerated Aging Tests. Journal of Composites for Construction, 2006. 10(4): p. 279-286.

55. Litherland, K.L., D.R. Oakley, and B.A. Proctor, The use of accelerated ageing procedures to predict the long term strength of GRC composites. Cement and Concrete Research, 1981. 11: p. 455-466.

56. Phani, K.K. and N.R. Bose, Temperature Dependence of Hydrothermal Ageing of CSM-Laminate During Water Immersion. Composites Science and Structures, 1987. 29: p. 7987.

57. Chen, Y., Accelerated Ageing Tests and Long-term Prediction Model for Durability of FRP Bars in Concrete, in Civil and Environmental Engineering. 2007, West Virginia University: Morgantown. p. 214.

58. Prian, L. and A. Barkatt, Degradation mechanism of fiber-reinforced plastics and its implications to prediction of long-term behavior. Material Science, 1999. 34: p. 3977-3989.

59. Nkurunziza, G., et al., Durability of GFRP bars: A critical review of the literature. Progress in Structural Engineering and Materials, 2005. 7: p. 194-209. 
60. Karbhari, V.M., M. Engineer, and D.A.E. II, On the durability of composite rehabilitation schemes for concrete: use of a peel test. Materials Science, 1997. 32: p. 147-156.

61. Abanilla, M.A., Y. Li, and V.M. Karbhari, Durability characterization of wet layup graphite/epoxy composites used in external strengthening. Composites: Part B, 2006 a. 37: p. 200-212.

62. Karbhari, V.M. and M.A. Abanilla, Design factors, reliability, and durability prediction of wet layup carbon/epoxy used in external strengthening. Composites: Part B, 2007. 38: p. 10-23.

63. Wan, B., M.F. Petrou, and K.A. Harries, The Effect of the Presence of Water on the Durability of Bond between CFRP and Concrete. Reinforced Plastics and Composites, 2006. 25(8): p. 875-890.

64. Ouyang, Z. and B. Wan, Experimental and Numerical Study of Moisture Effects on the Bond Fracture Energy of FRP/Concrete Joints. Journal of REINFORCED PLASTICS AND COMPOSITES, 2008 a. 27(2): p. 205-223.

65. Mindness, S., J.F. Young, and D. Darwin, Concrete. 2 ed. 2002: Prentice Hall.

66. Delaney, J. and V. Karbhari. Defect criticality in FRP strengthening. in 8th Int. Symp. in Fiber-Reinforced (FRP) Polymer Reinforcement for Concrete Structures (FRPRCS-8). 2007. University of Patras, Greece.

67. Shen, X., et al. Effect of surface roughness on the bond performance between FRP laminates and concrete. in 2nd International Conference on Durability of Fiber Reinforced Polymer (FRP) Composites for Construction. 2002. University of Sherbrooke, Canada.

68. Toutanji, H. and G. Oritz, The effect of surface preparation on the bond interface between FRP sheets and concrete members. Composite Structures, 2001. 53: p. 457-462.

69. Yalim, B., A.S. Kalayci, and A. Mirmiran, Performance of FRP-Strengthened RC Beams with Different Concrete Surface Profiles. Composites for Construction, 2008. 12(6): p. 626-634.

70. ACI, Design and construction of externally bonded FRP systems for strengthening concrete structures, in ACI 440.2R-02. 2002, American Concrete Institute: Farmington Hills, MI. 
71. ICRI, Selecting and Specifying Concrete Surface Preparation for Sealers, Coatings, and Polymer Overlays (Guideline No. 03732). January 1997, International Concrete Repair Institute (ICRI).

72. Xiao, J., J. Li, and Q. Zha, Experimental study on bond behavior between FRP and concrete Construction and Building Materials, 2004. 18(10): p. 745-752

73. Au, C. and O. Büyüköztürk, Peel and Shear Fracture Characterization of Debonding in FRP Plated Concrete Affected by Moisture. Composites for Construction, 2006. 10(1): p. 35-47.

74. Maaddawy, T.E. and K. Soudki, Carbon-Fiber-Reinforced Polymer Repair to Extend Service Life of Corroded Reinforced Concrete Beams. Composites for Construction, 2005. 9(2): p. 187-194.

75. Renka, R.J. and A.K. Cline, A Triangle-Based $C^{1}$ Interpolation Method. Rocky Mountain Journal of Mathematics, 1984. 14(1): p. 223-237.

76. Davalos, J.F., S.S. Kodkani, and I. Ray, Fracture Mechanics Method for Mode-I Interface Evaluation of FRP Bonded to Concrete Substrates. Materials in Civil Engineering, 2006. 18(5): p. 732-742. 


\section{Appendices}


Appendix A: Strain-
Location Curves 

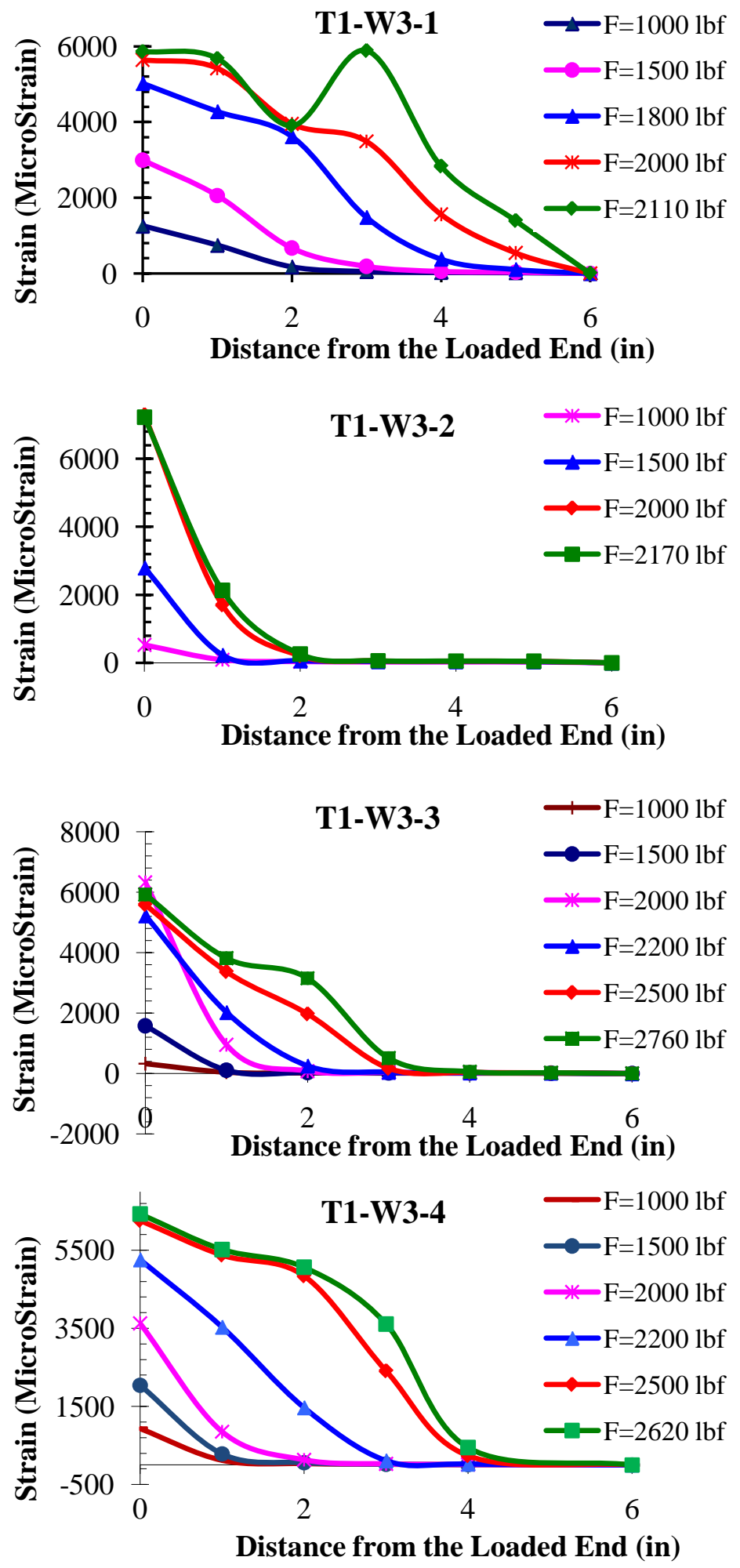

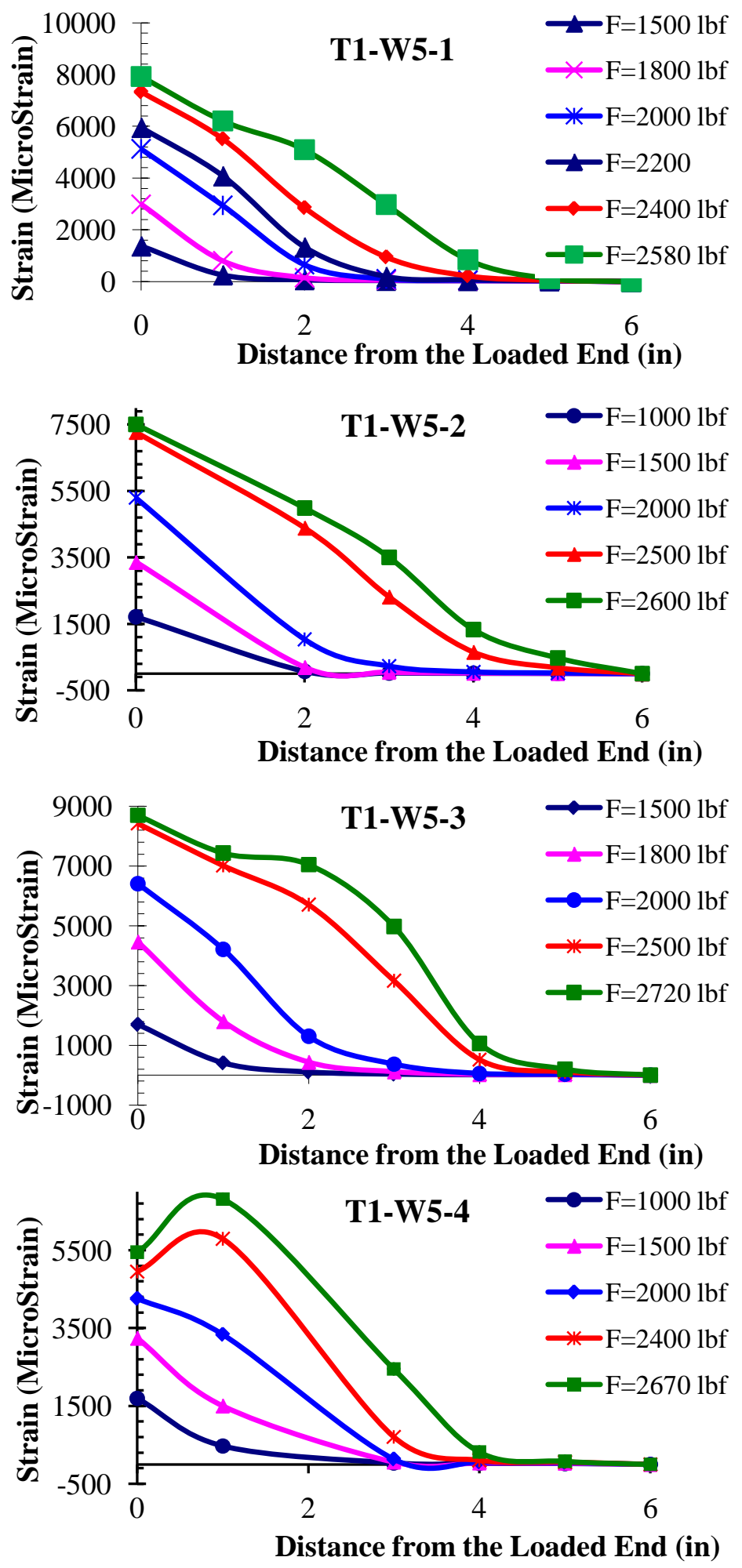

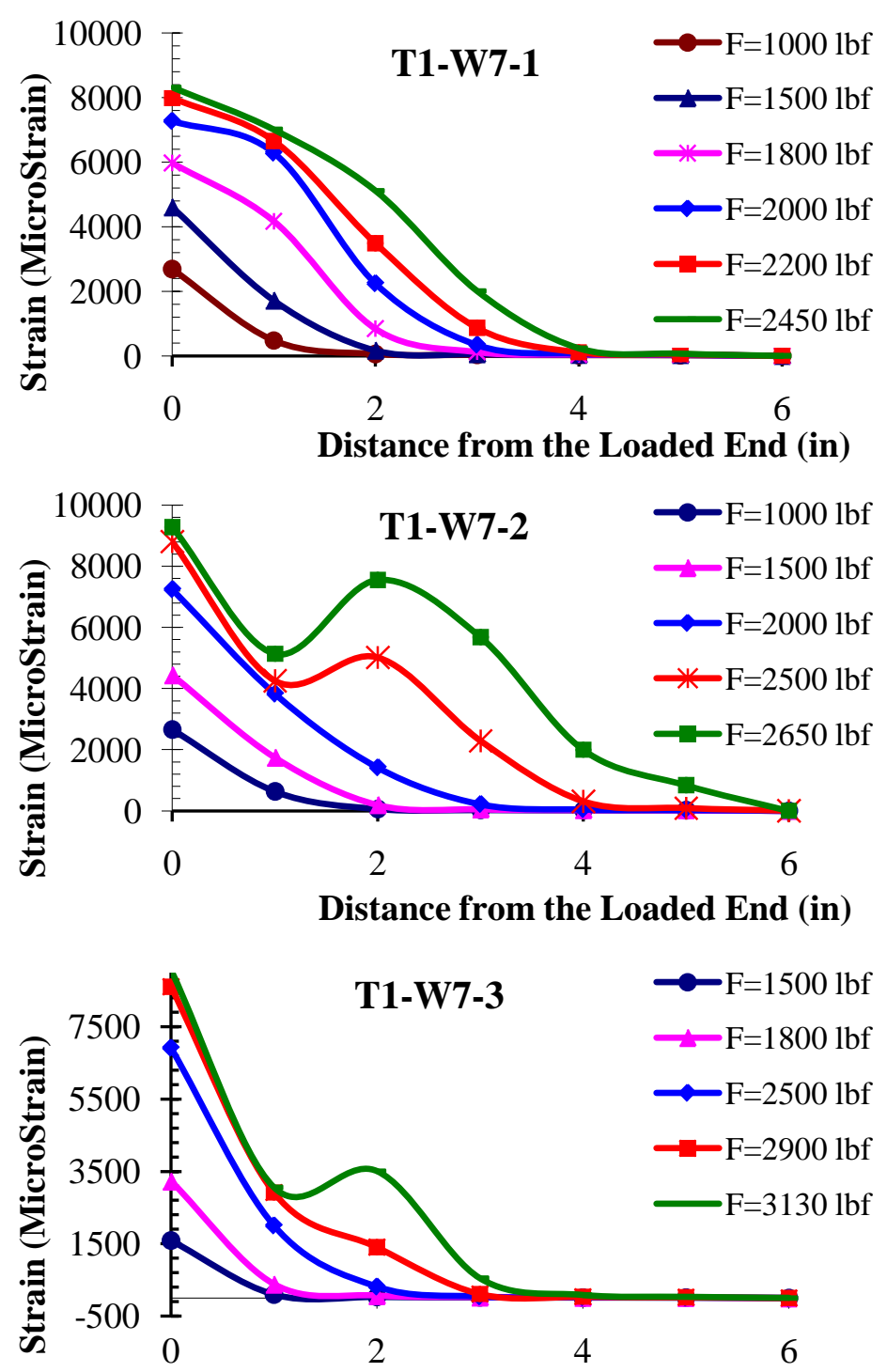

Distance from the Loaded End (in)

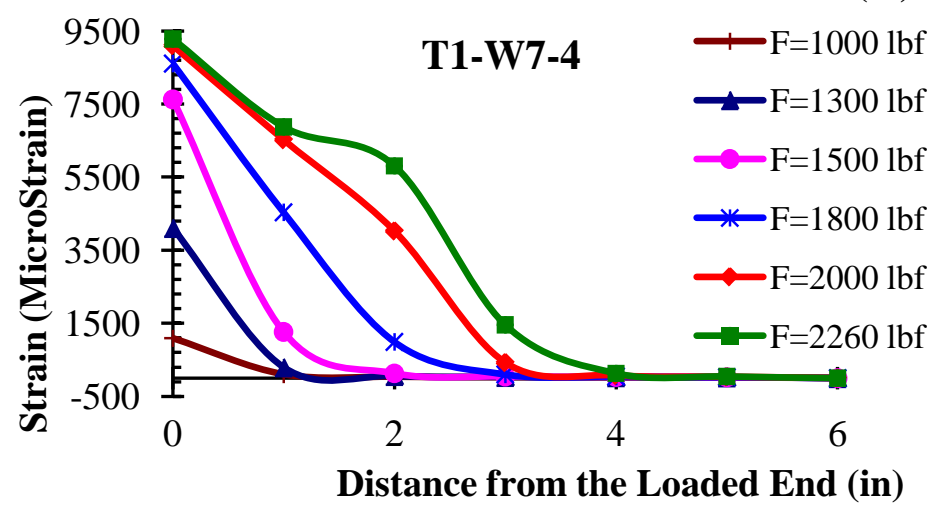




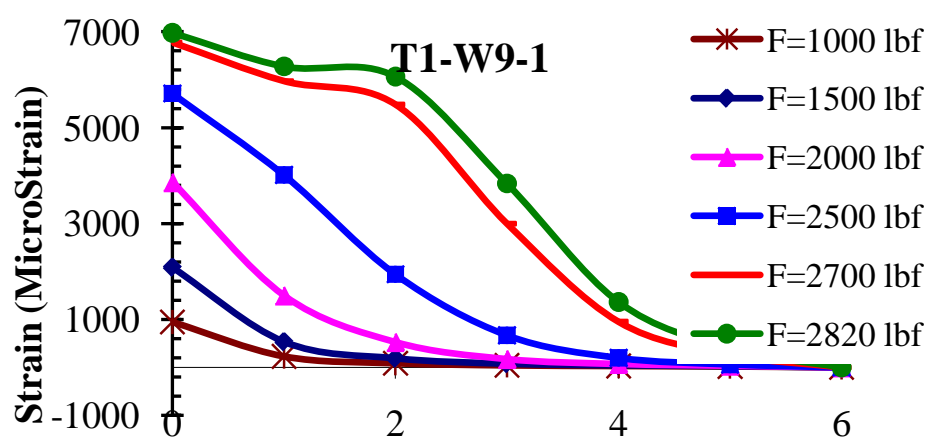

Distance from the Loaded End (in)

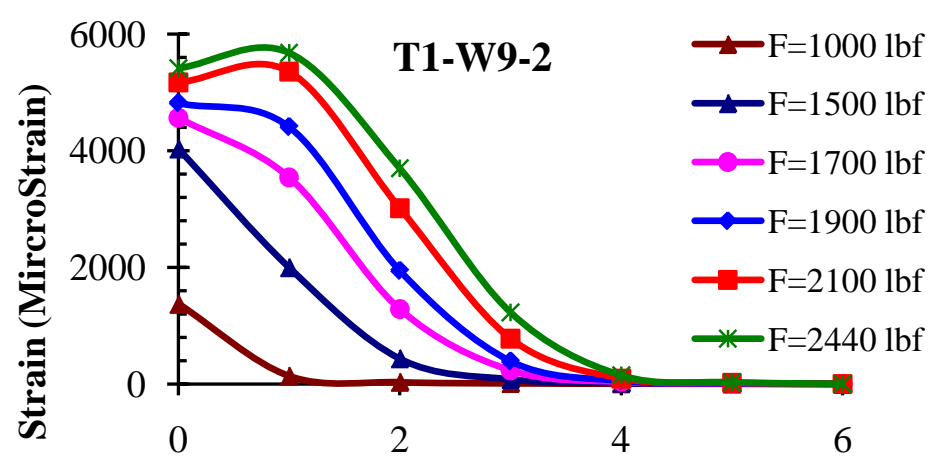

Distance from the Loaded End (in)
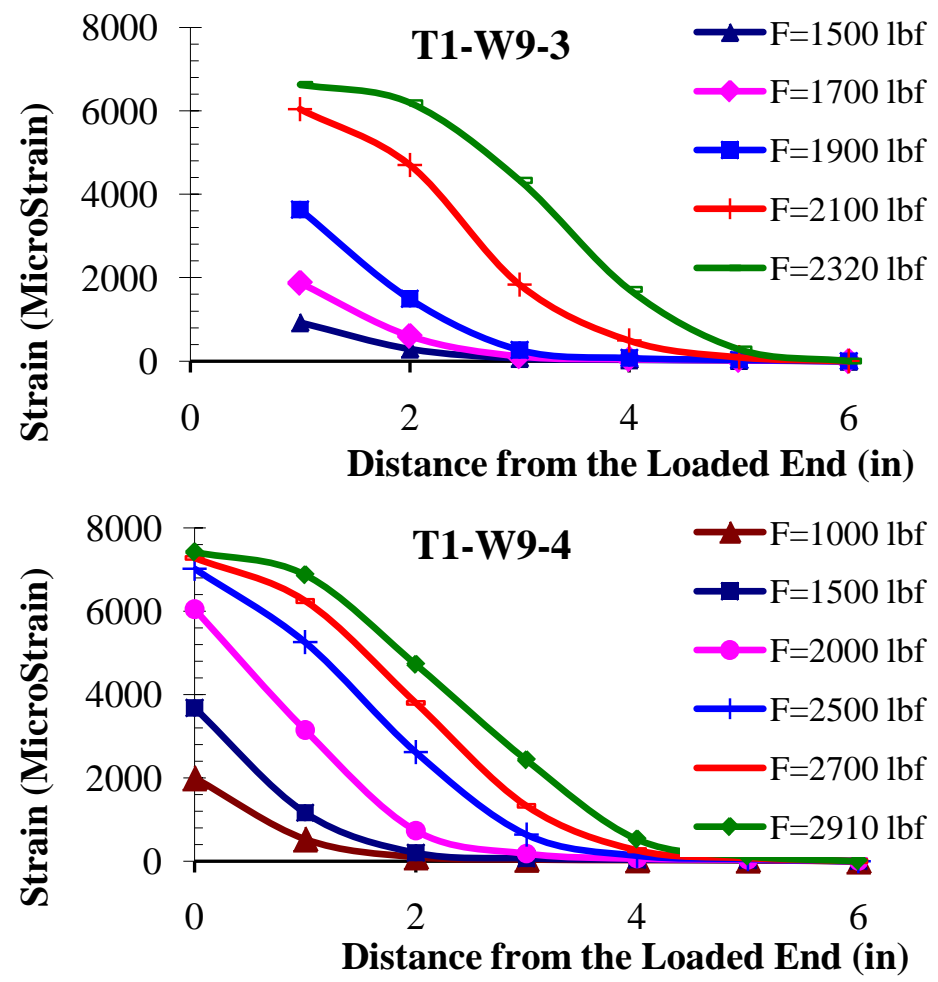


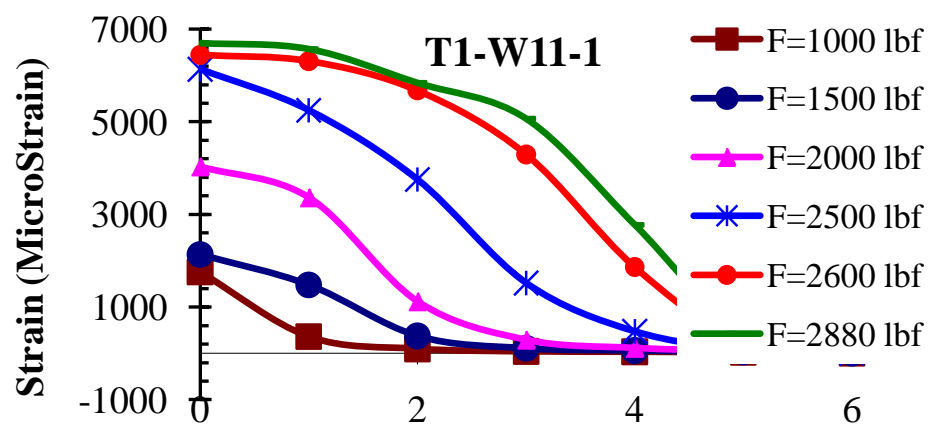

Distance from the Loaded End (in)
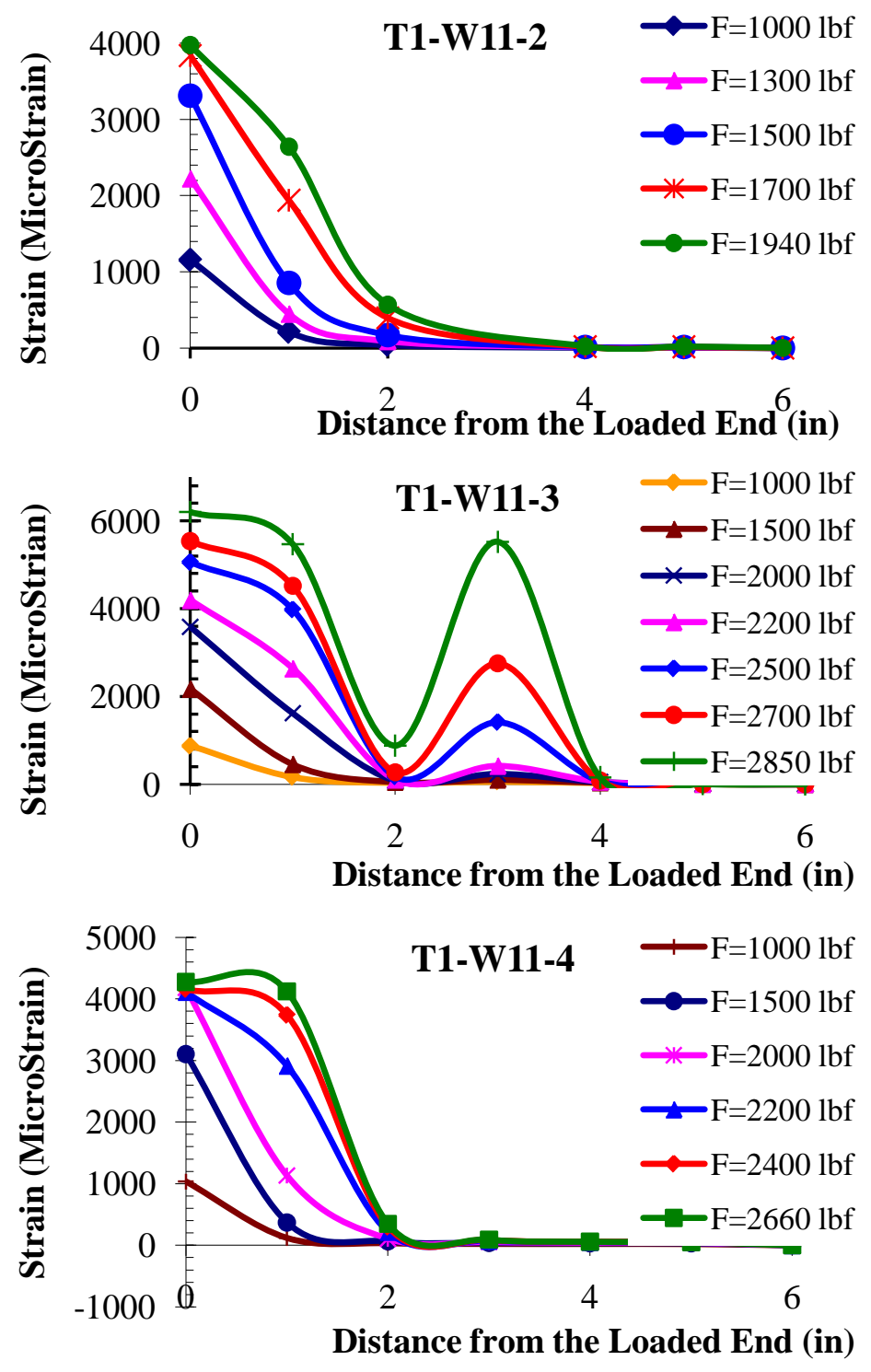

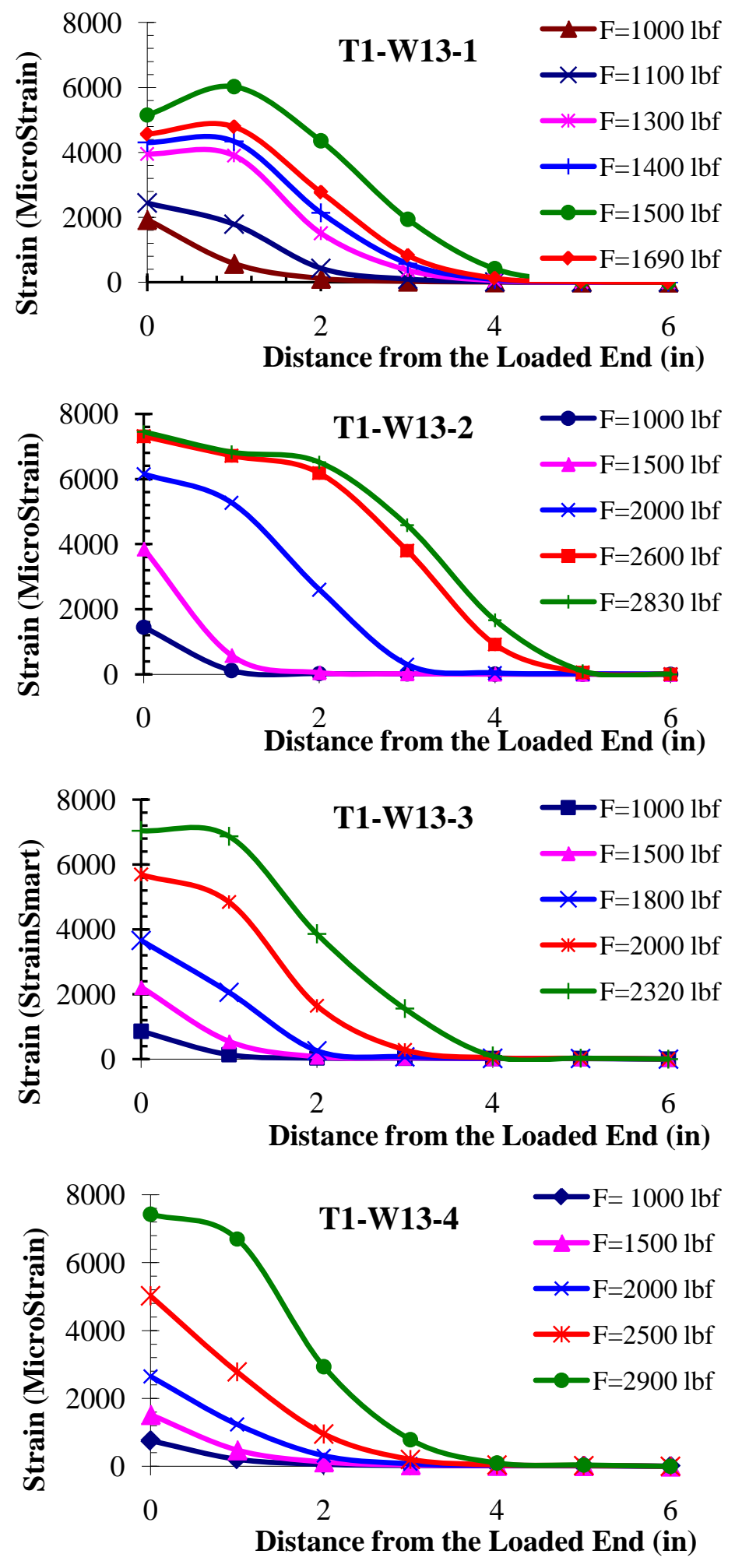

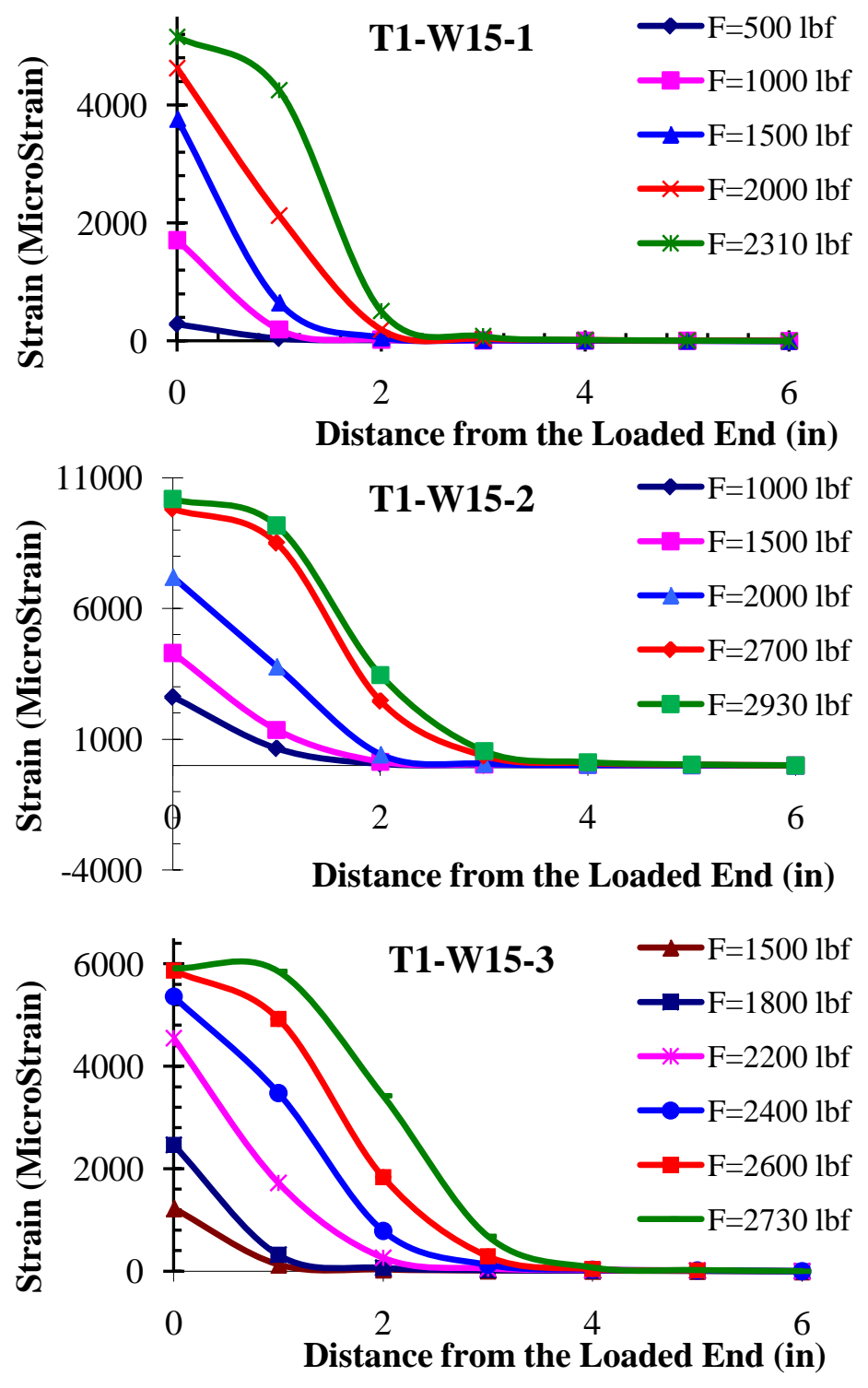

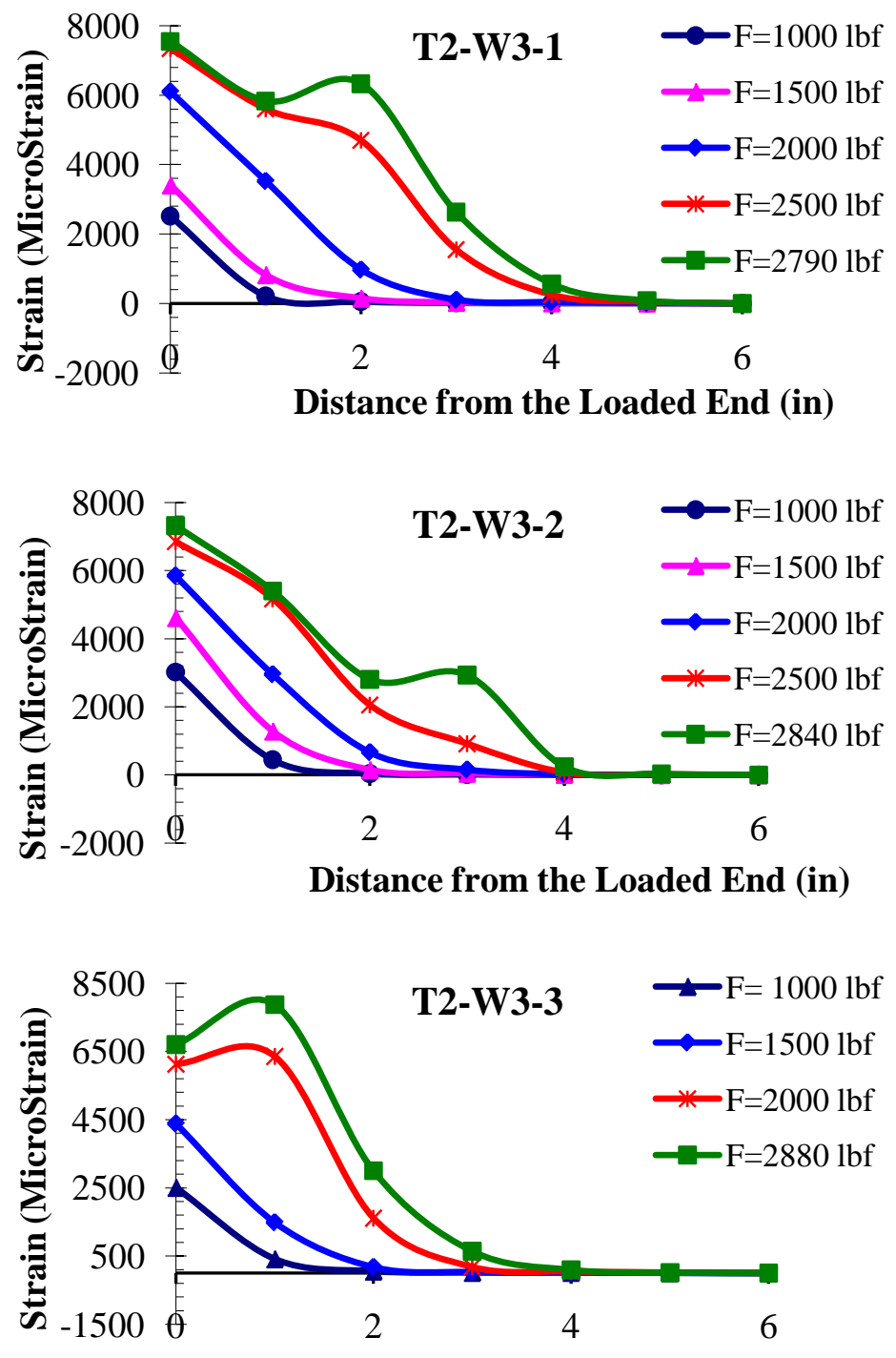

Distance from the Loaded End (in)

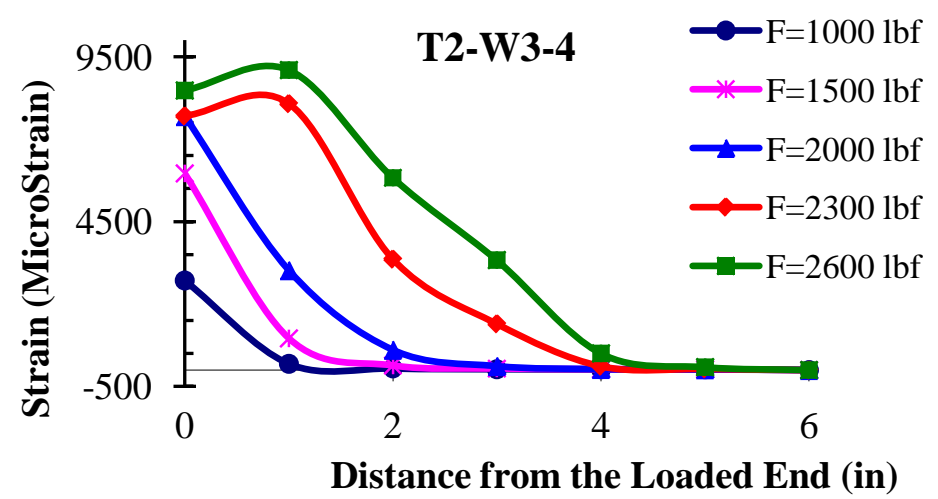




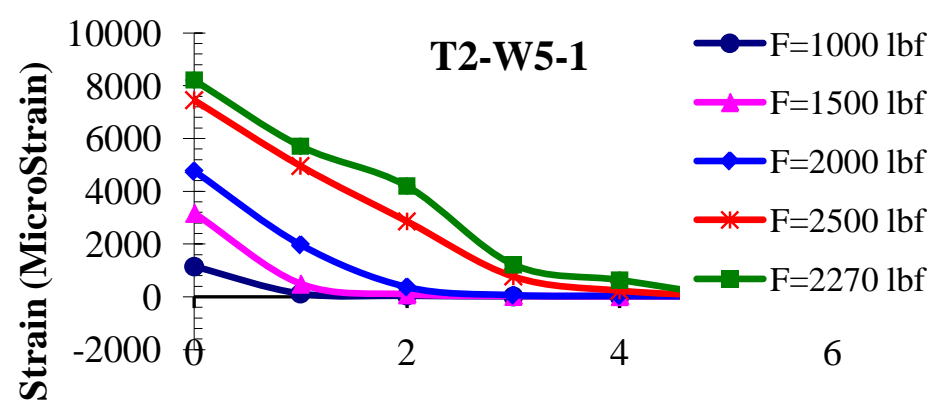

Distance from the Loaded End (in)

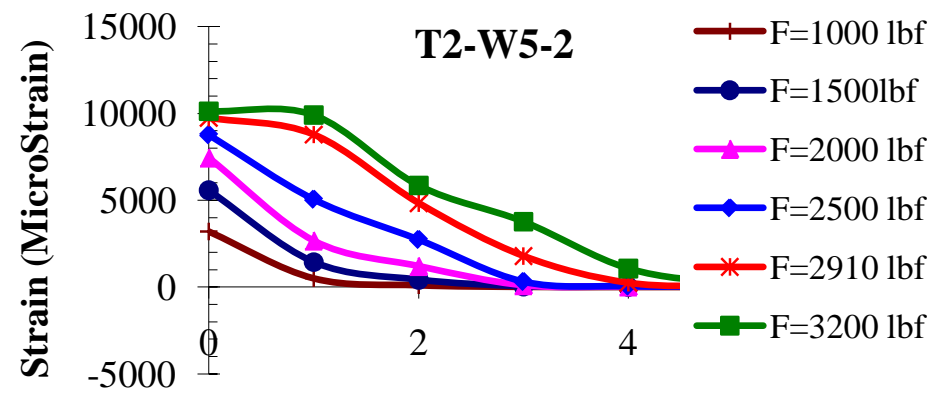

Distance from the Loaded End (in)
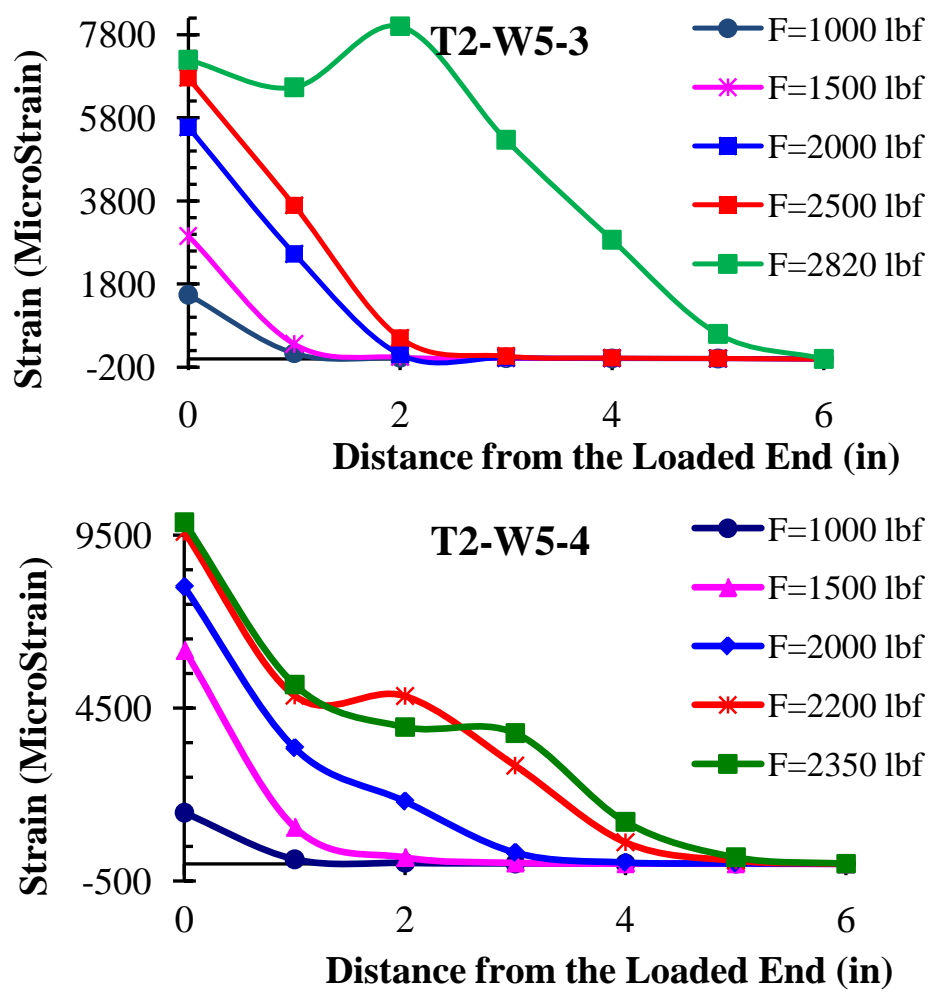

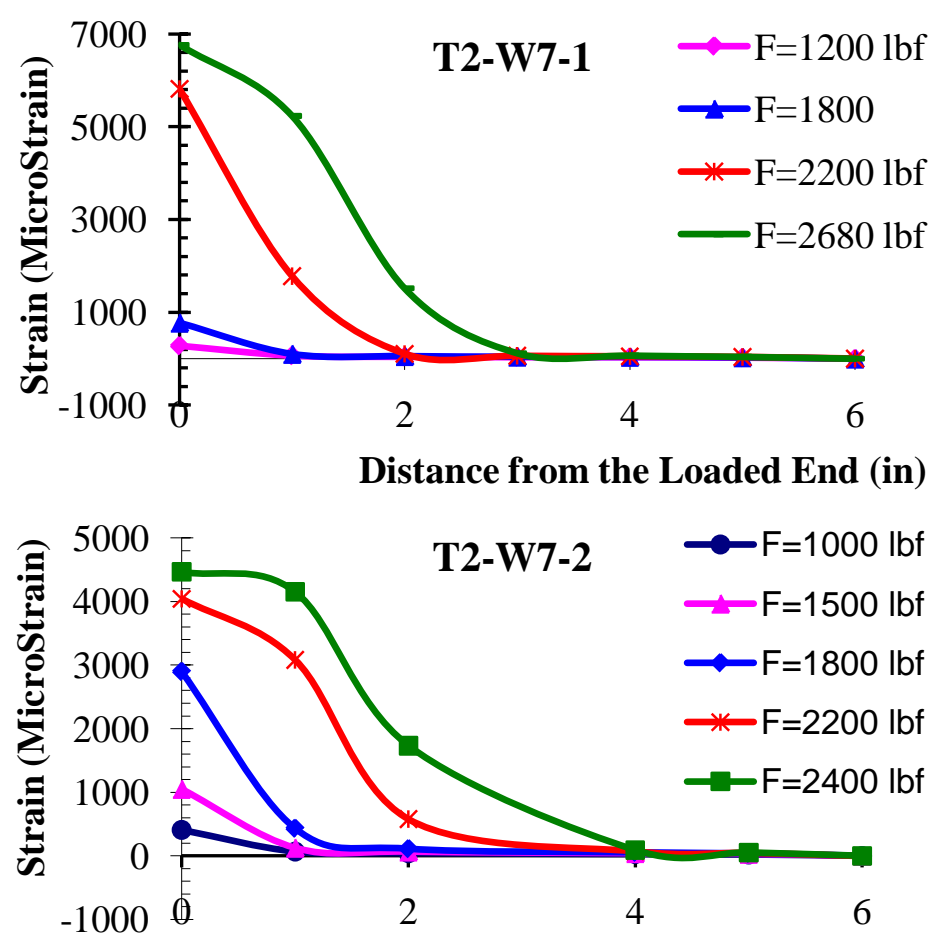

Distance from the Loaded End (in)
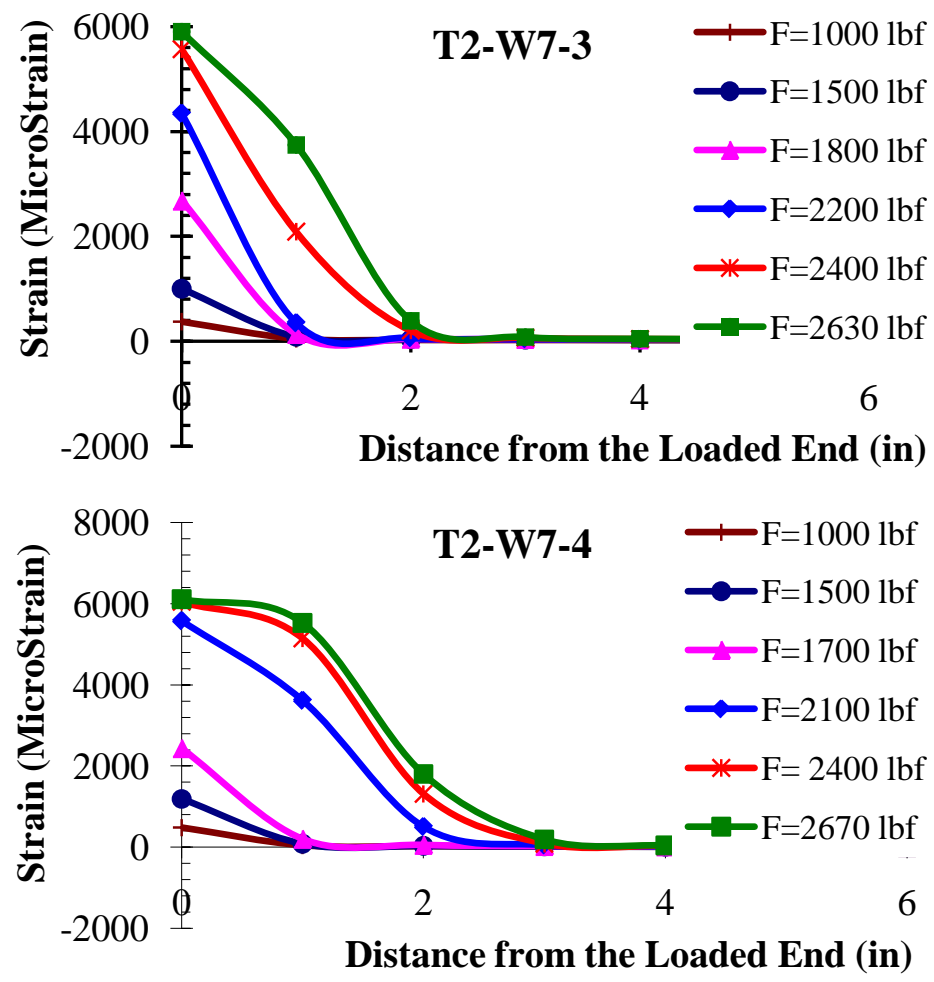

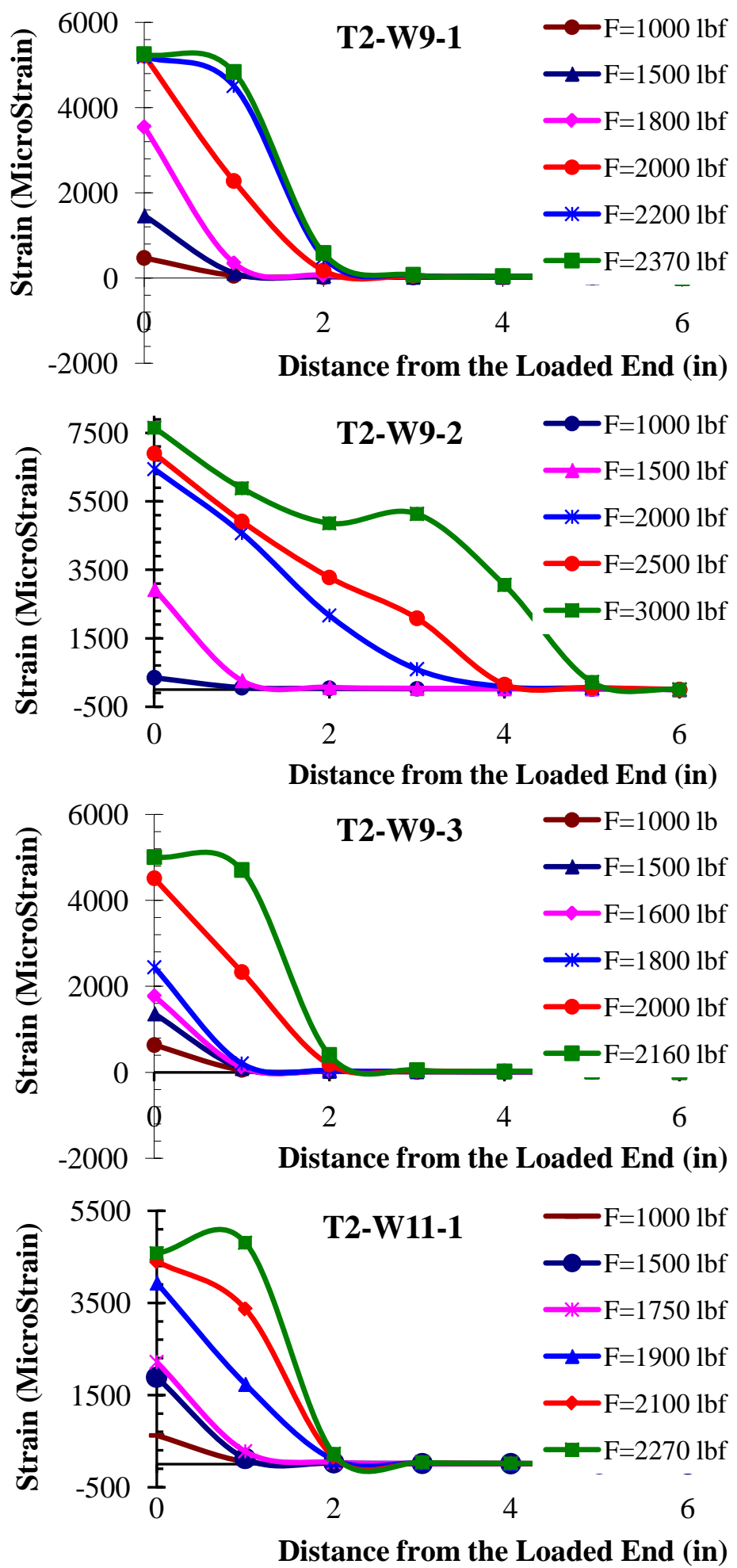

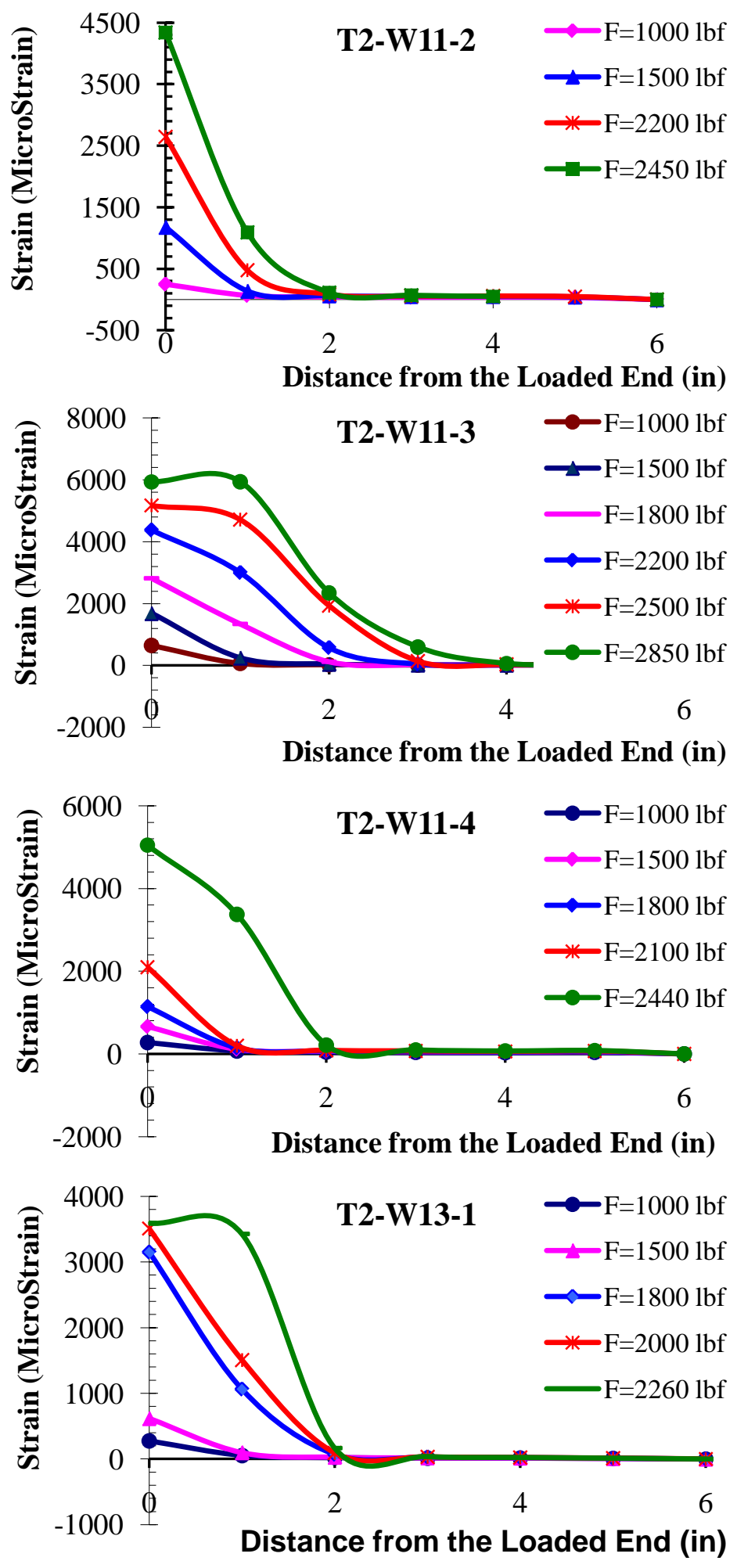

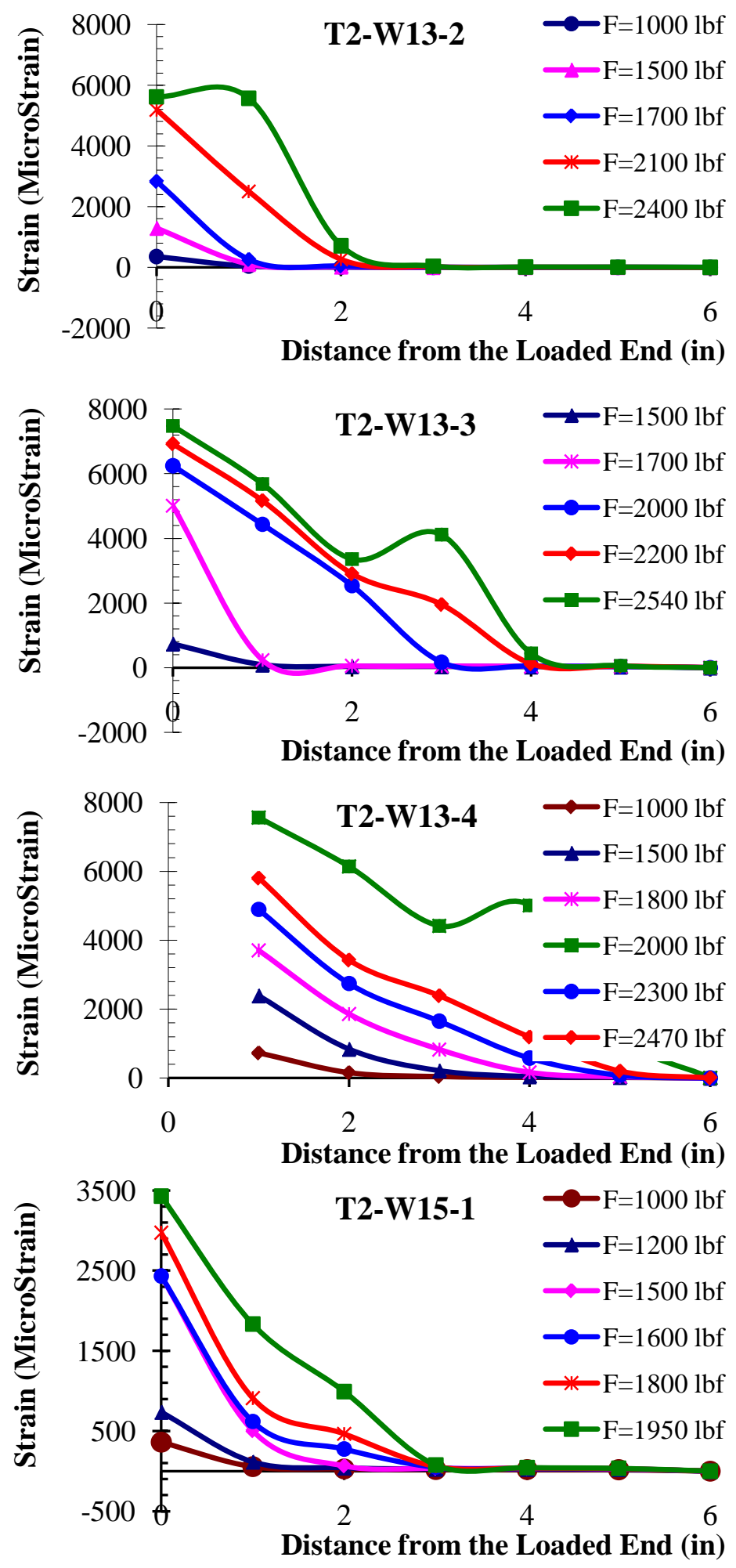

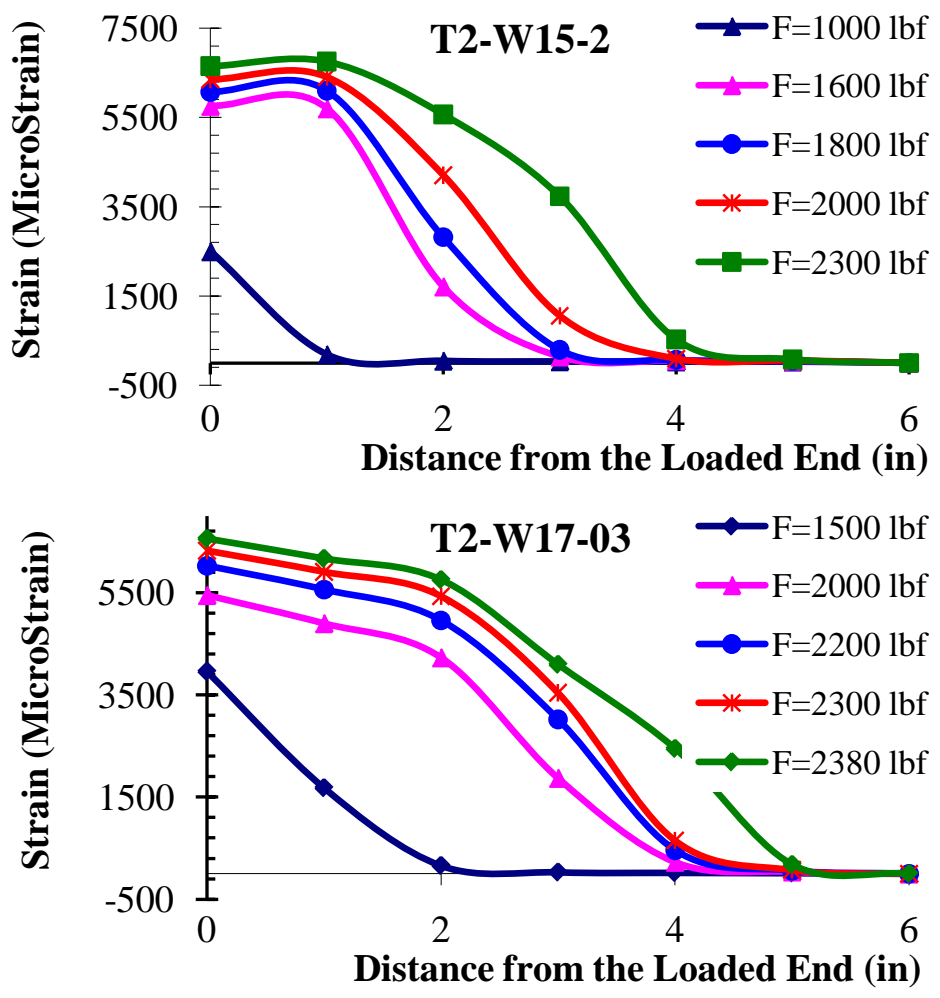

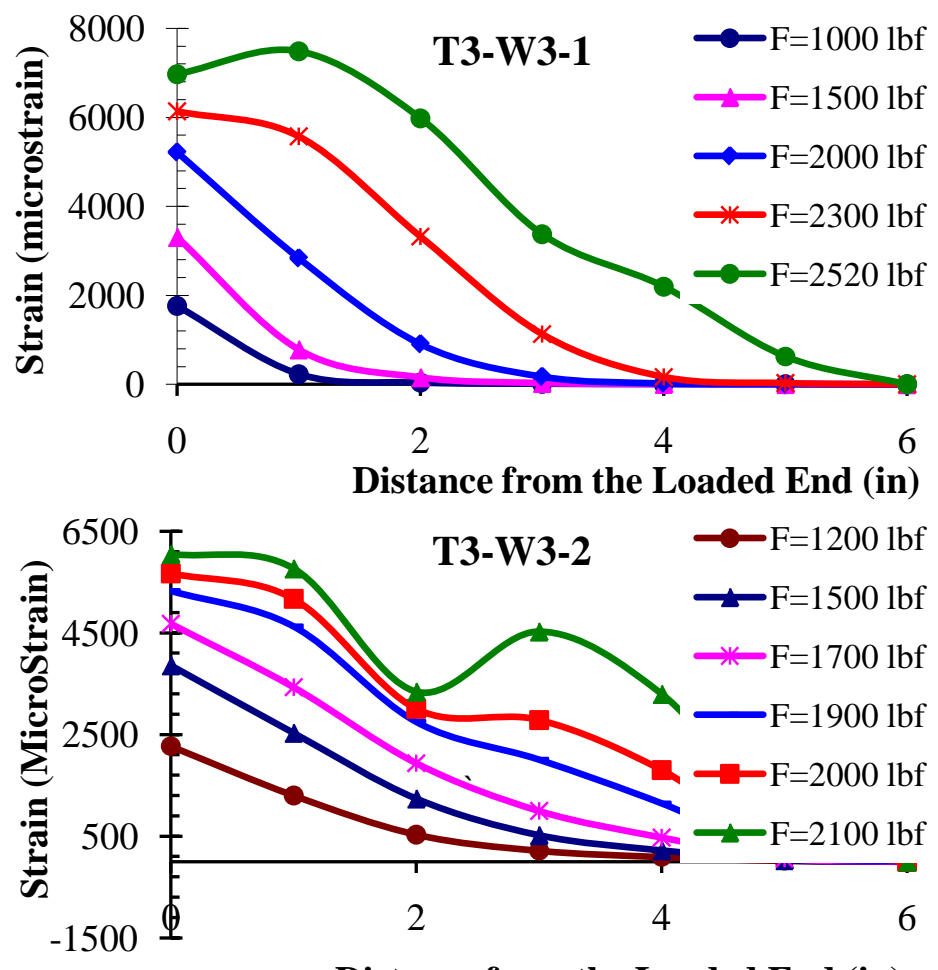

Distance from the Loaded End (in)

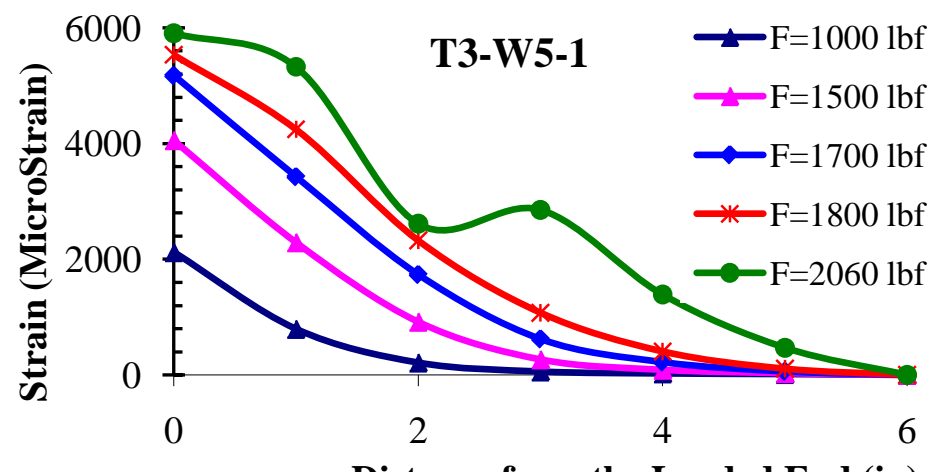

Distance from the Loaded End (in)

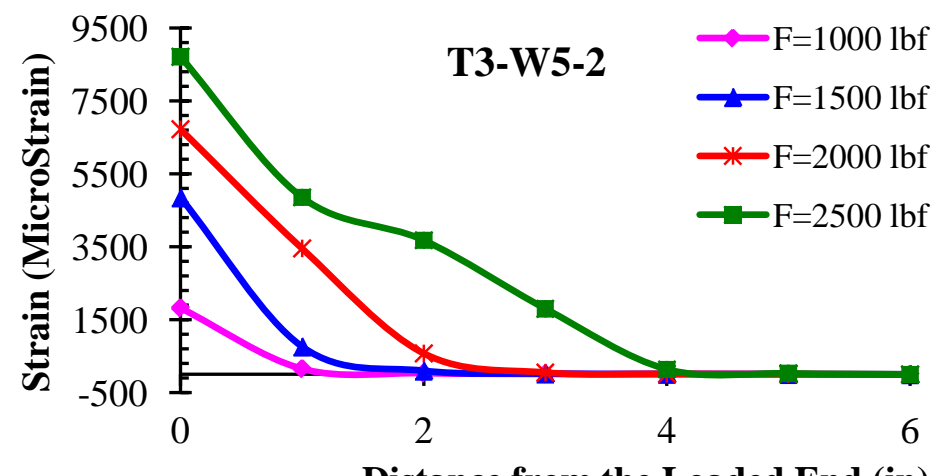

Distance from the Loaded End (in) 


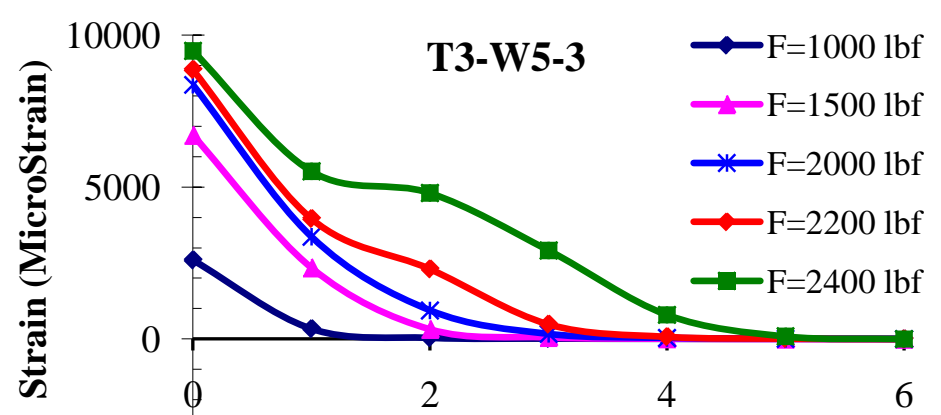

Distance from the Loaded End (in)

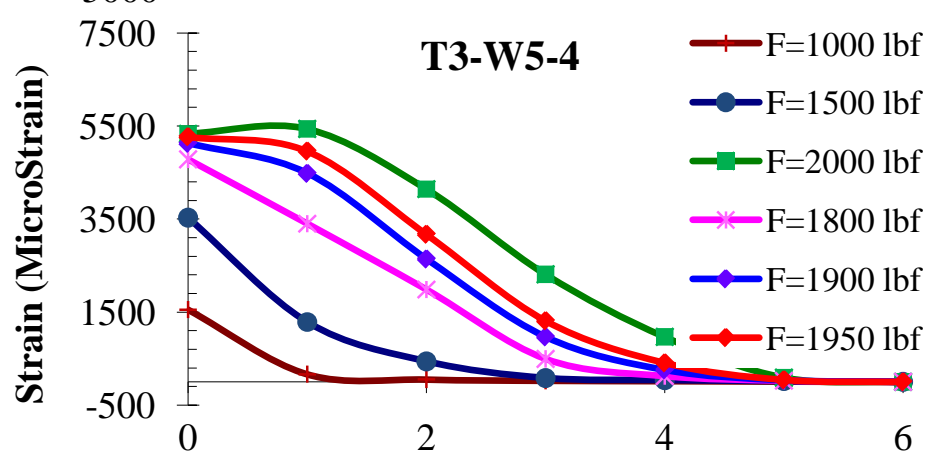

Distance from the Loaded End (in)
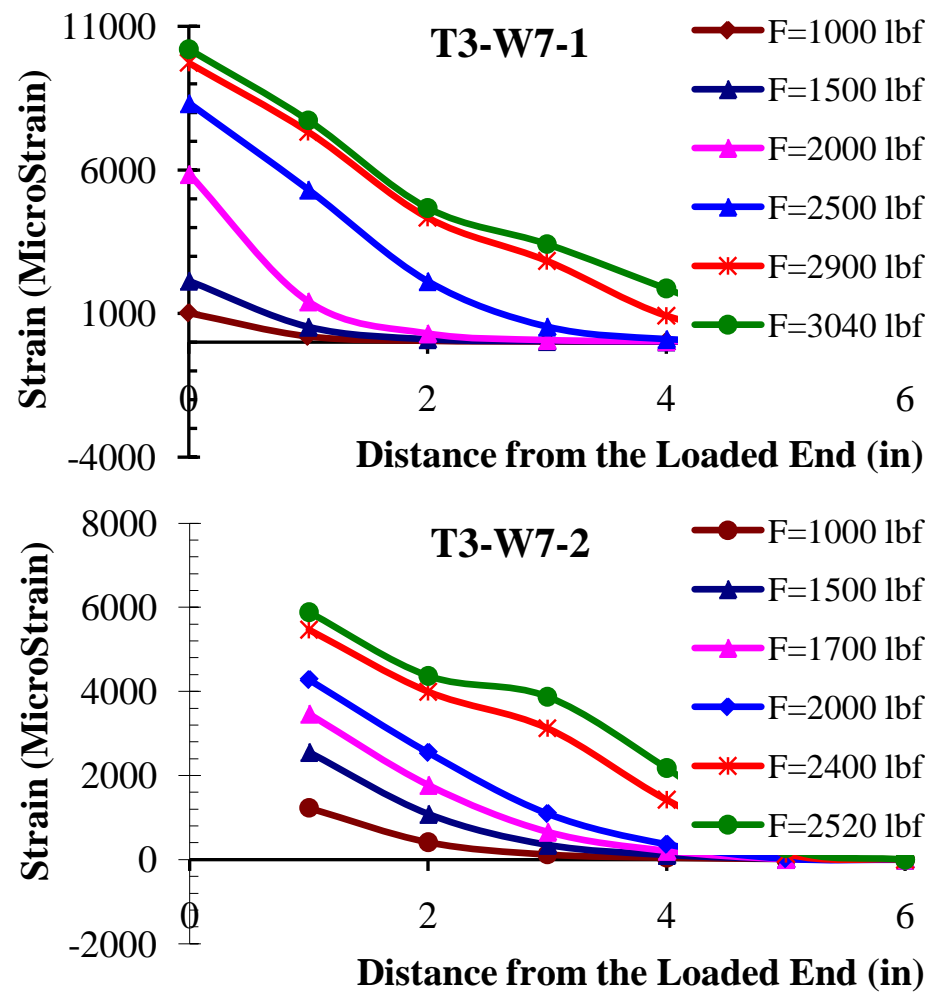

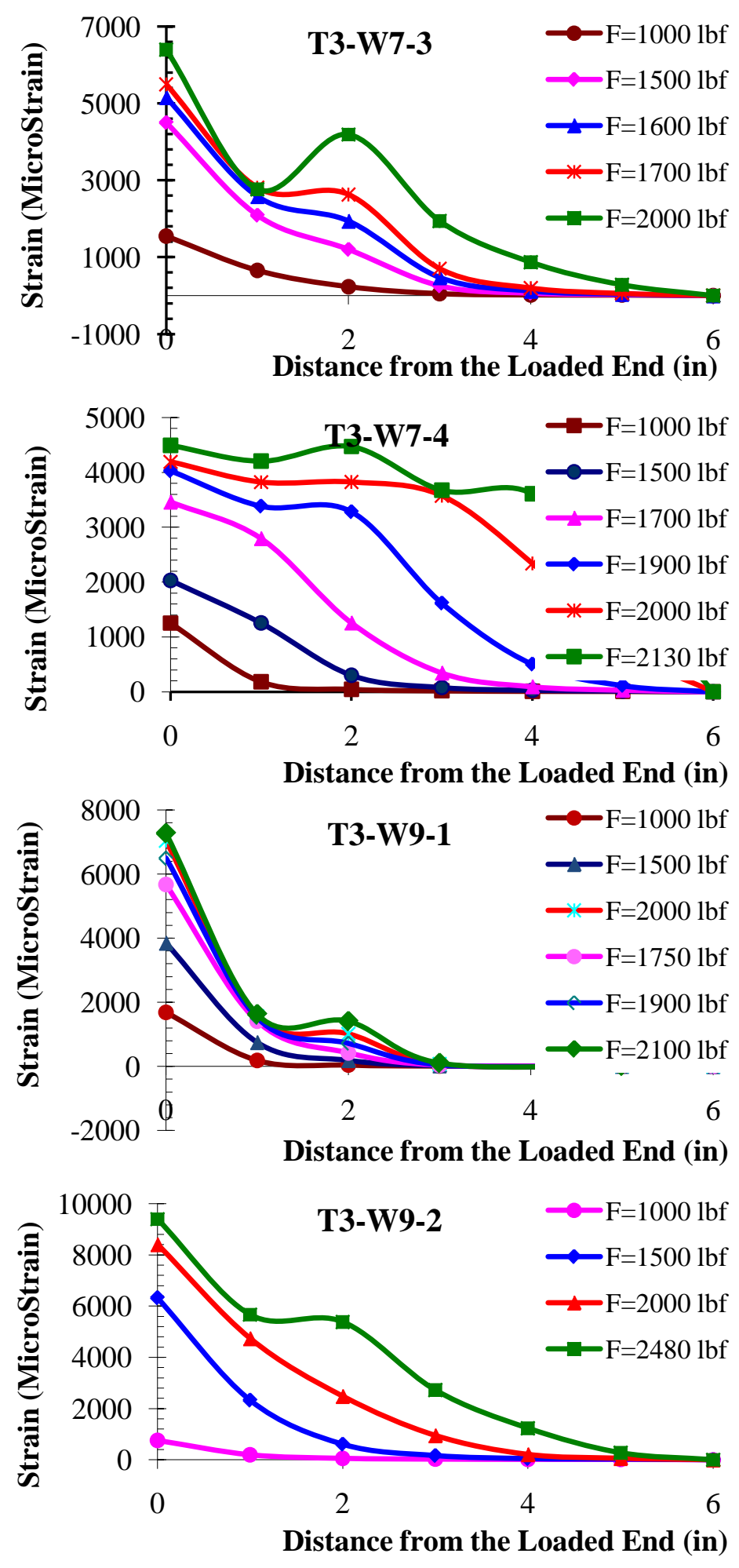

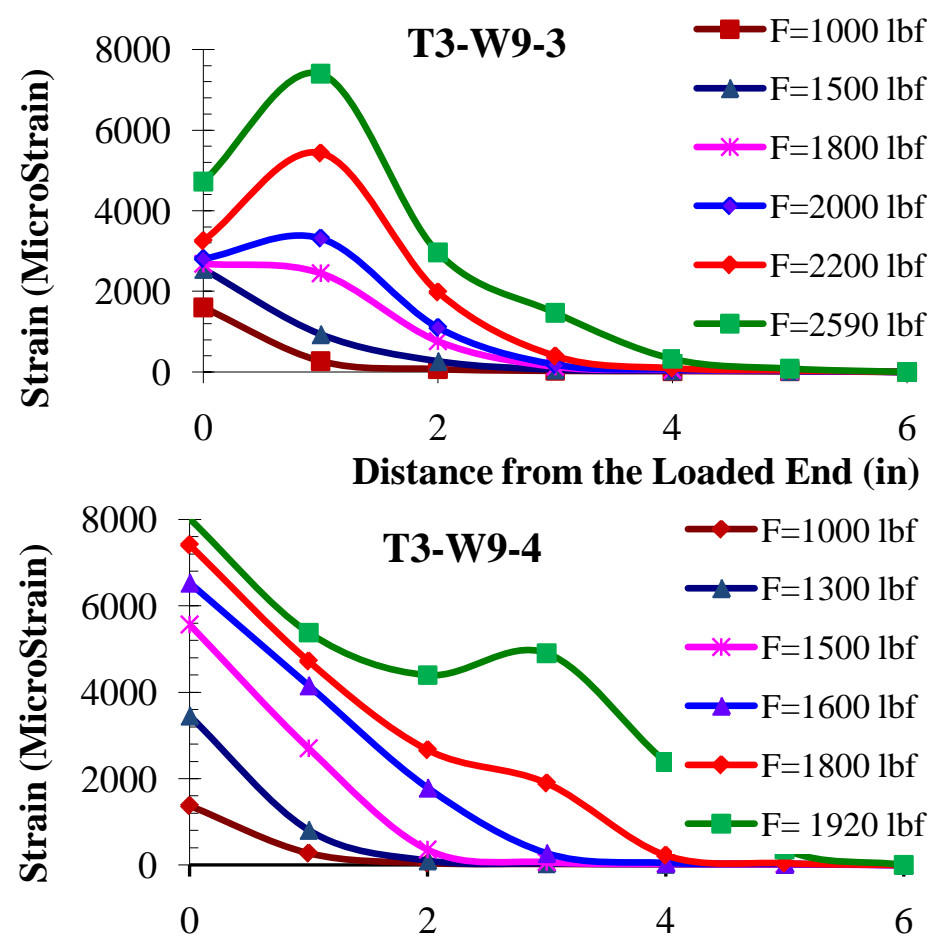

Distance from the Loaded End (in)
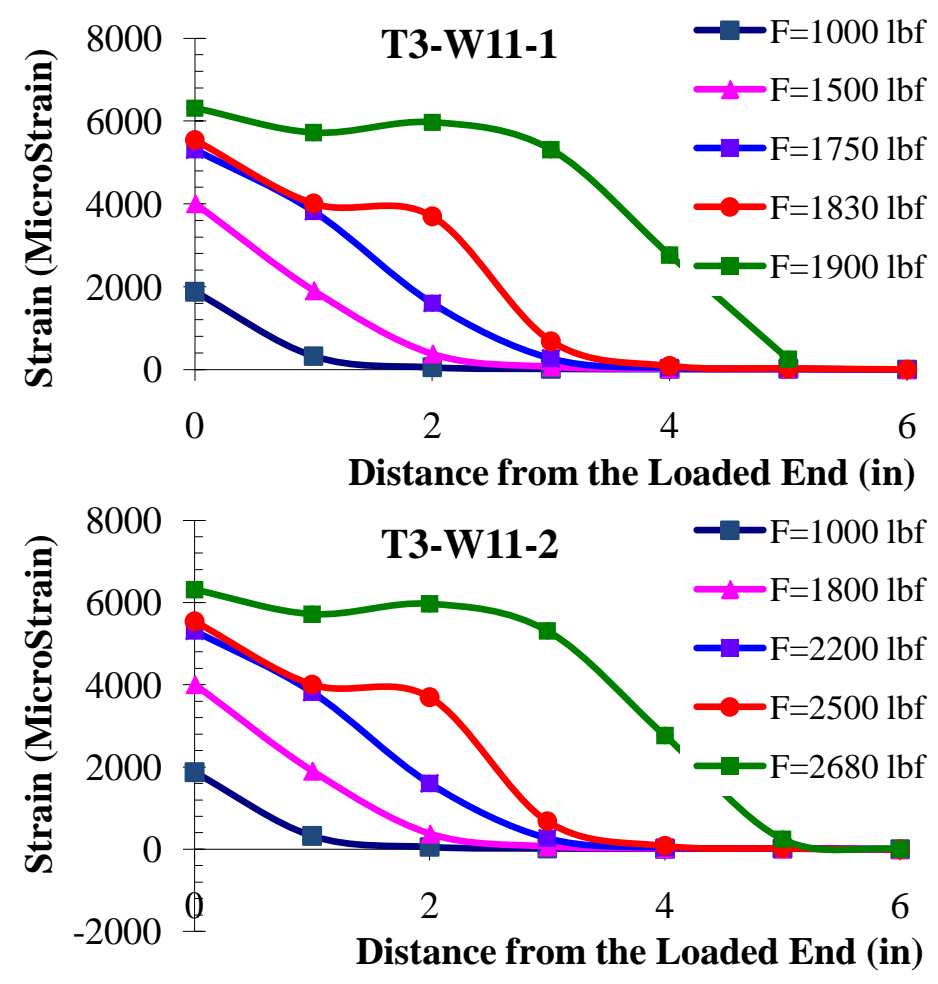


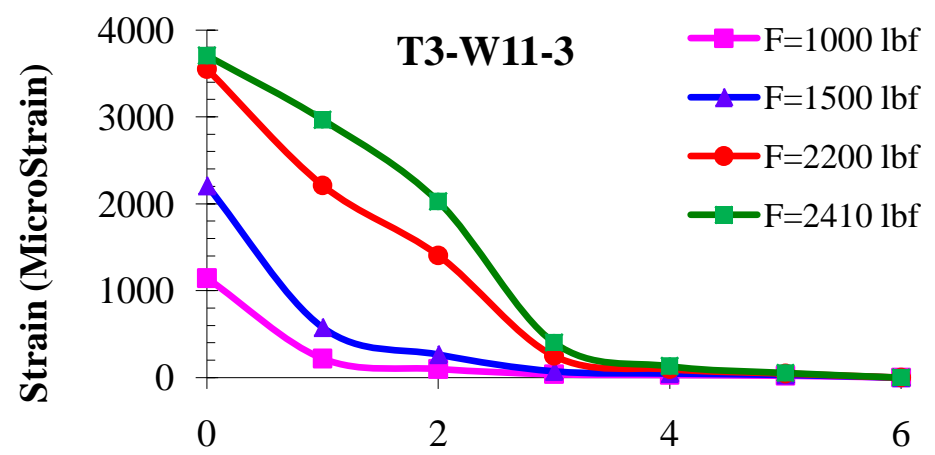

Distance from the Loaded End (in)

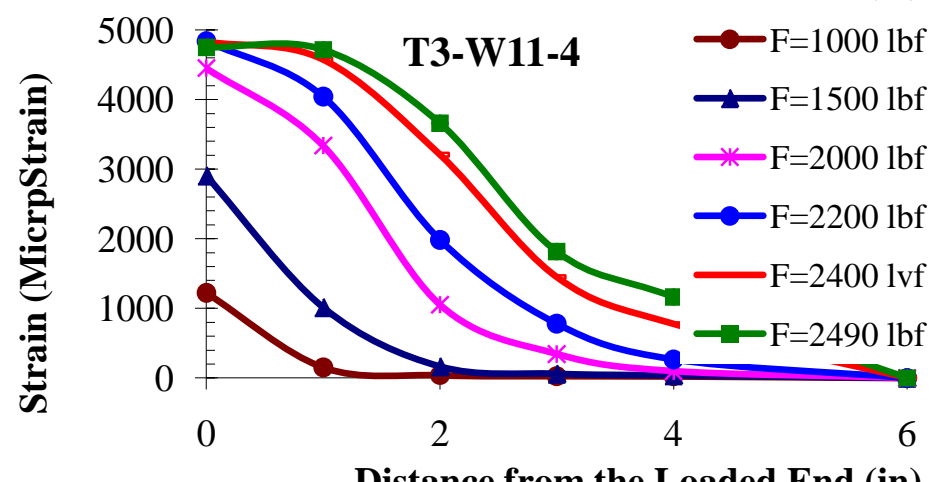

Distance from the Loaded End (in)
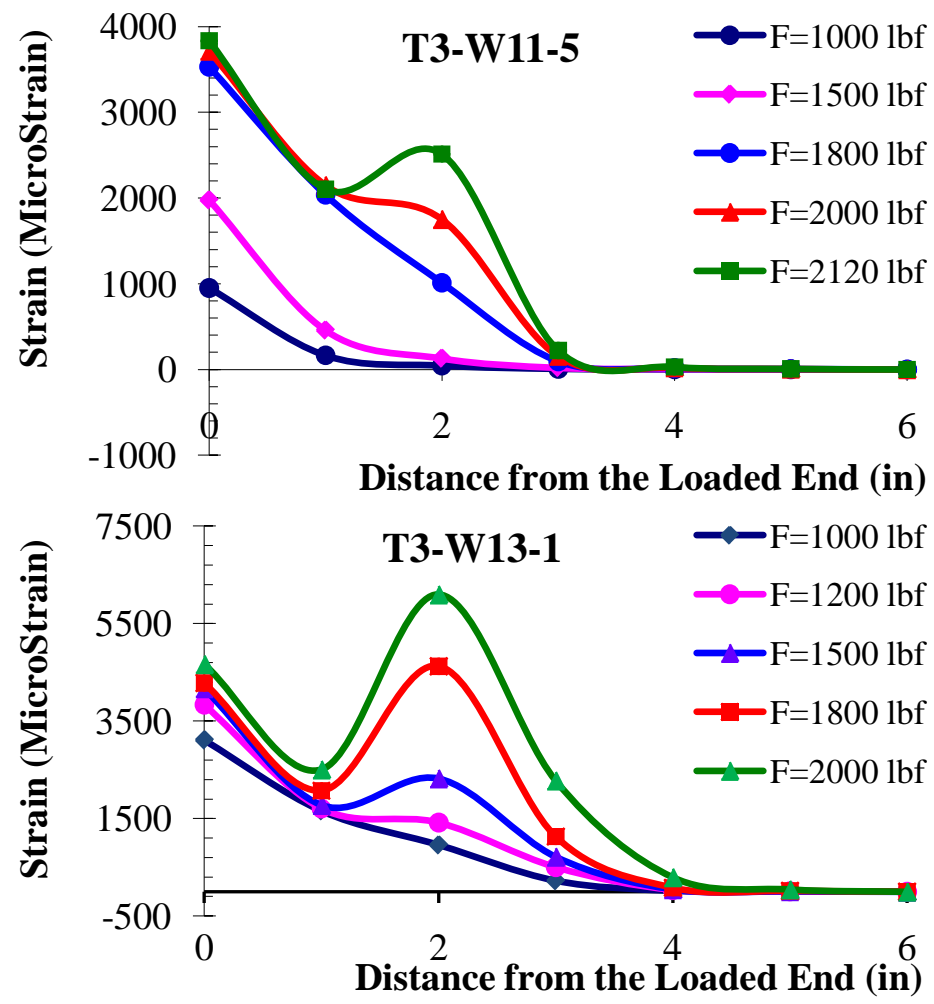


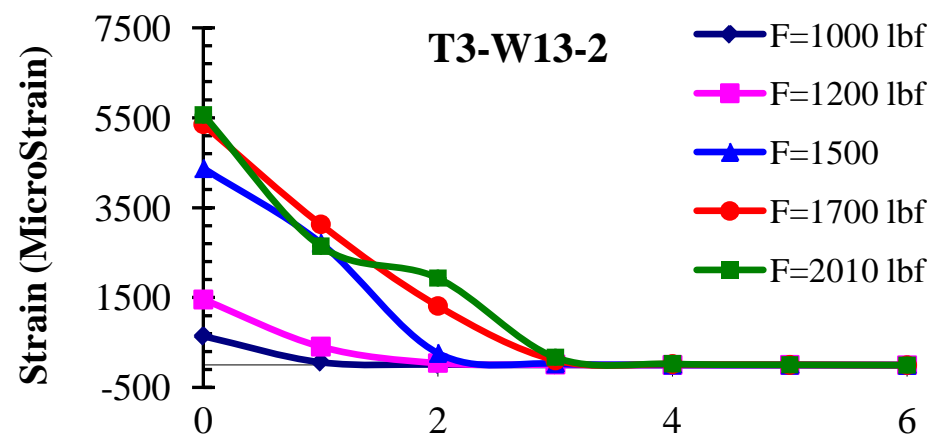

Distance from the Loaded End (in)

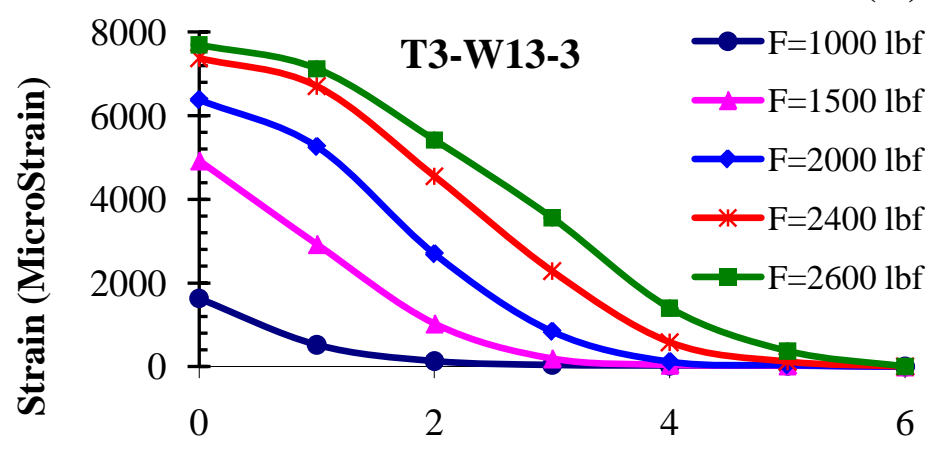

Distance from the Loaded End (in)
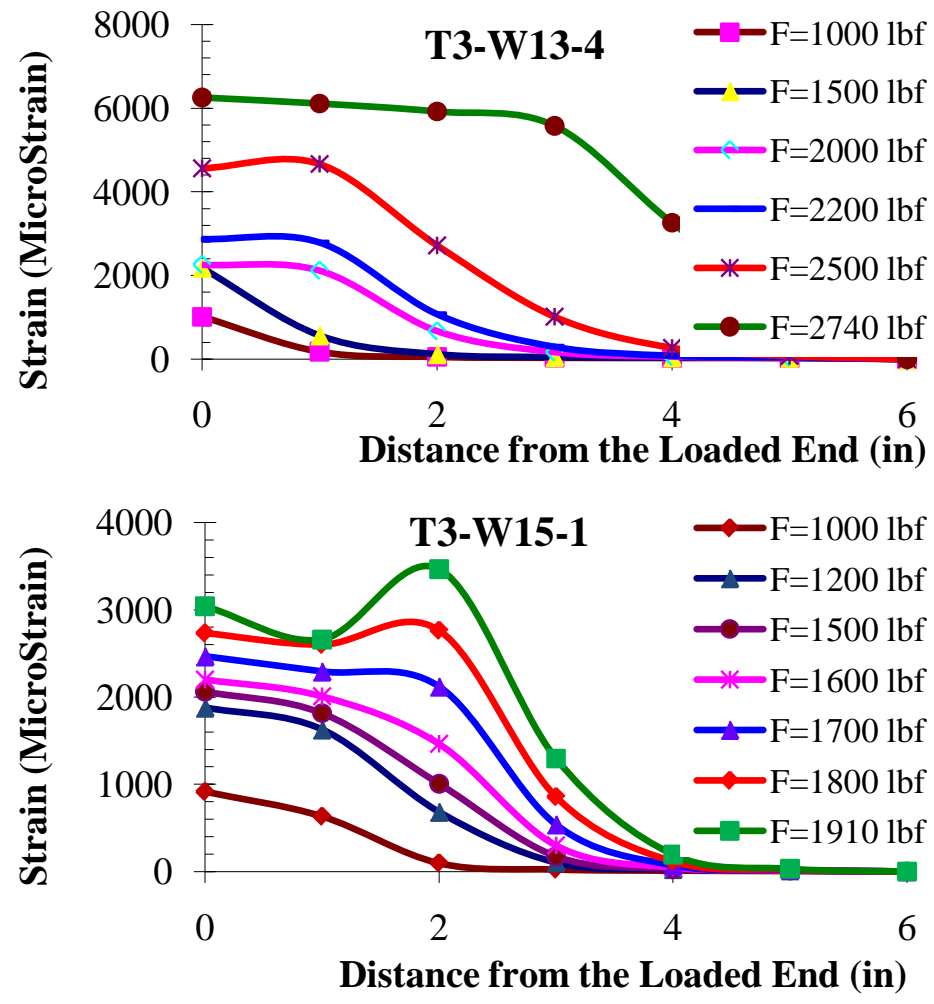

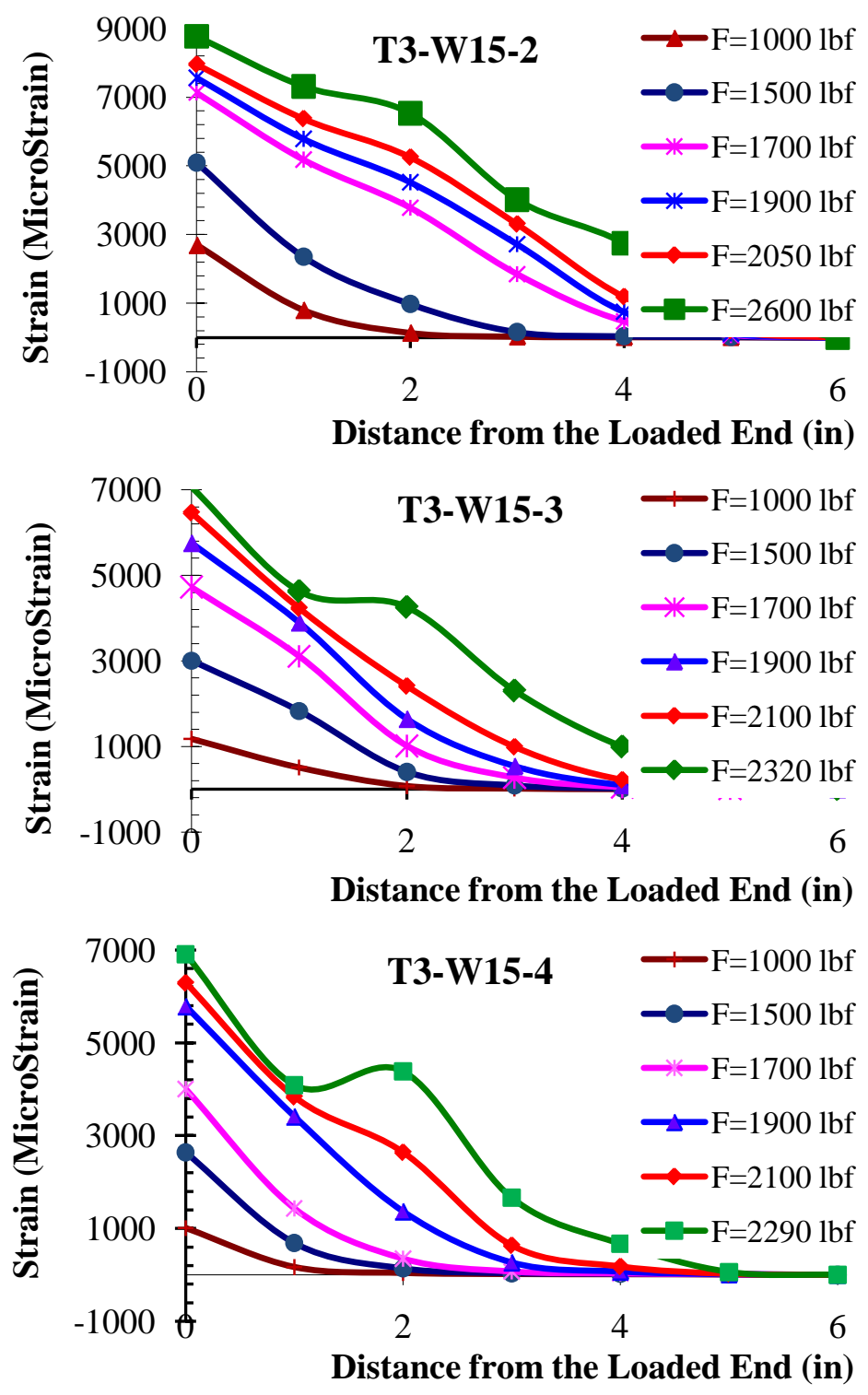


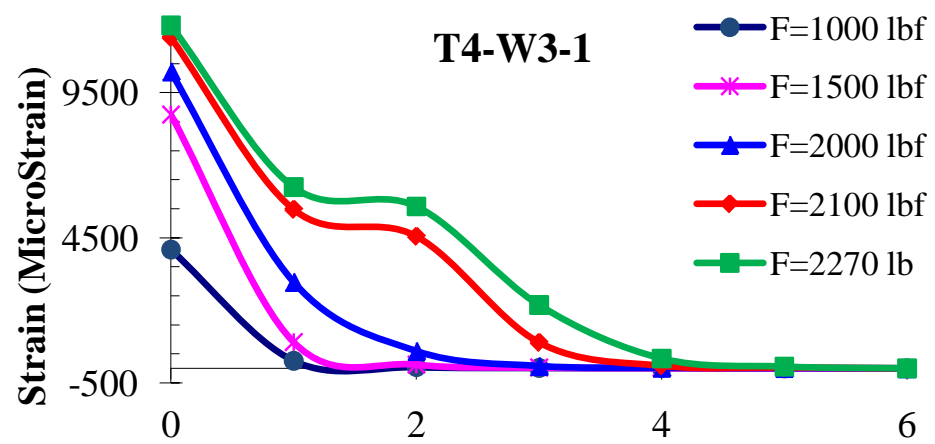

Distance from the Loaded End (in)

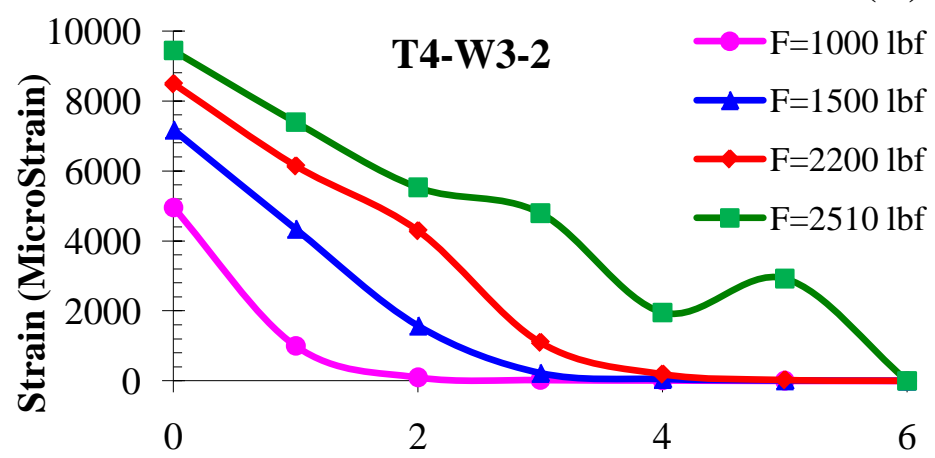

Distance from the Loaded End (in)

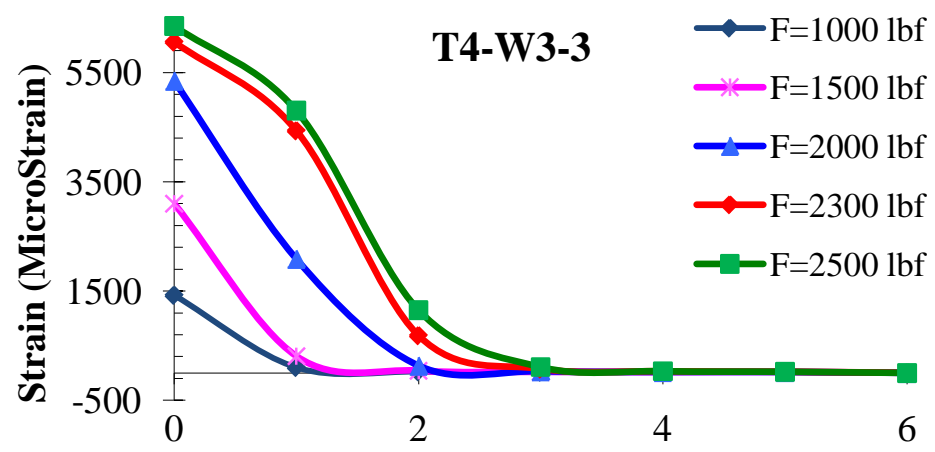

Distance from the Loaded End (in)

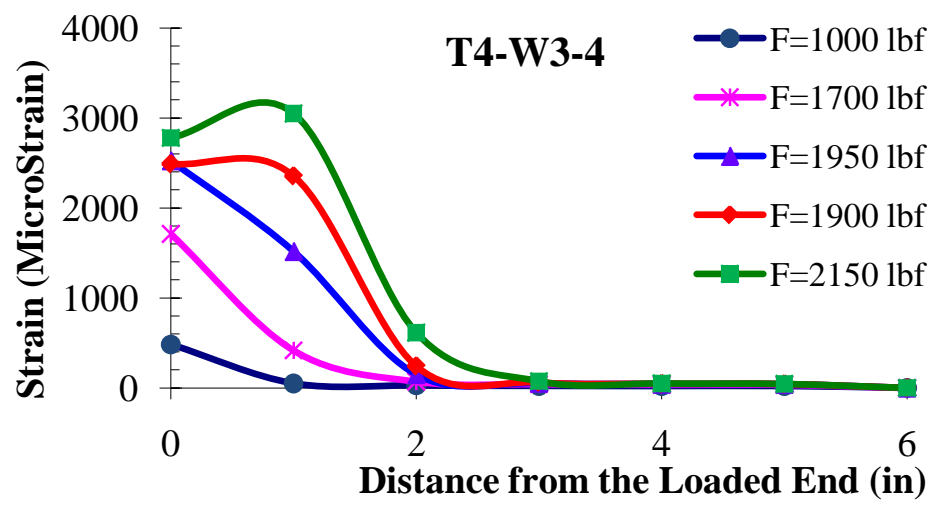



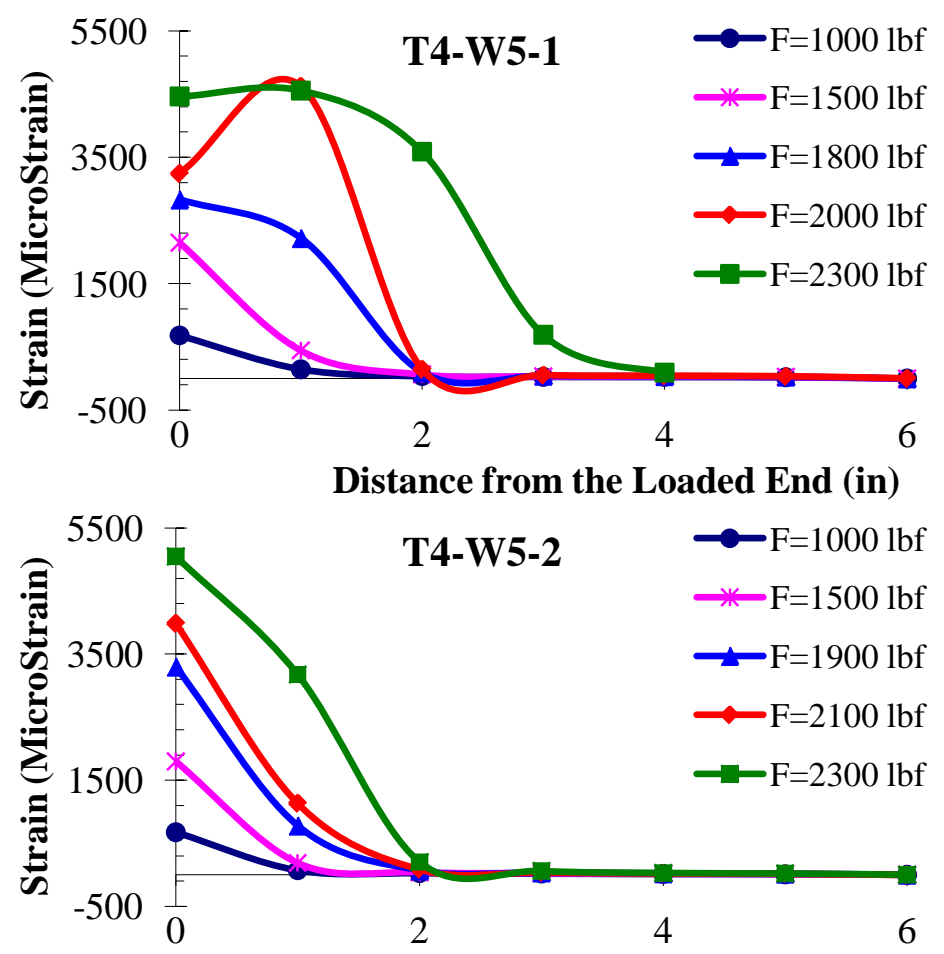

Distance from the Loaded End (in)
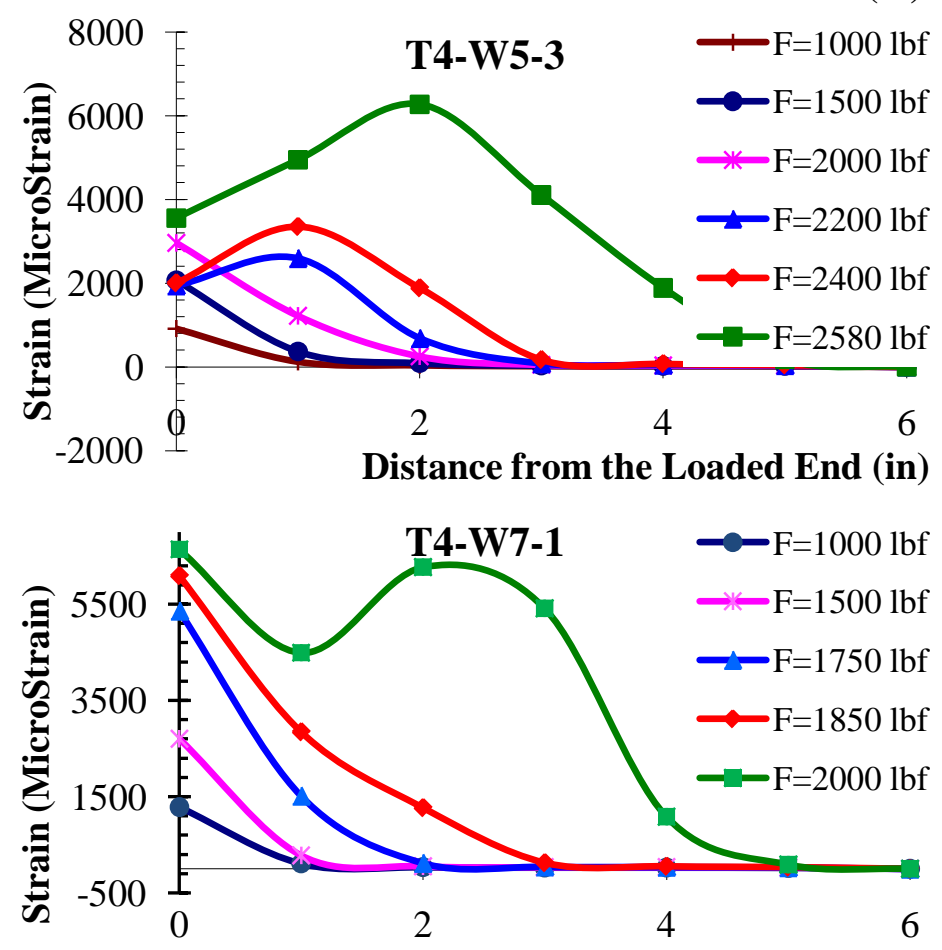

Distance from the Loaded End (in) 


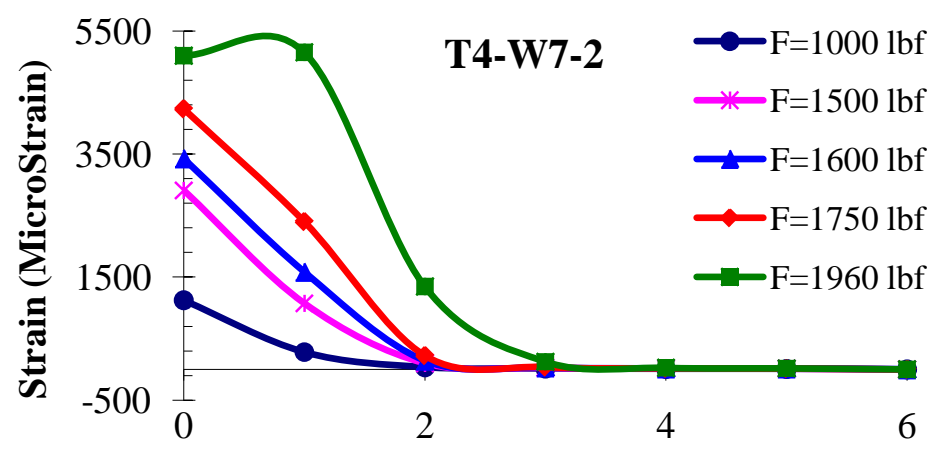

Distance from the Loaded End (in)
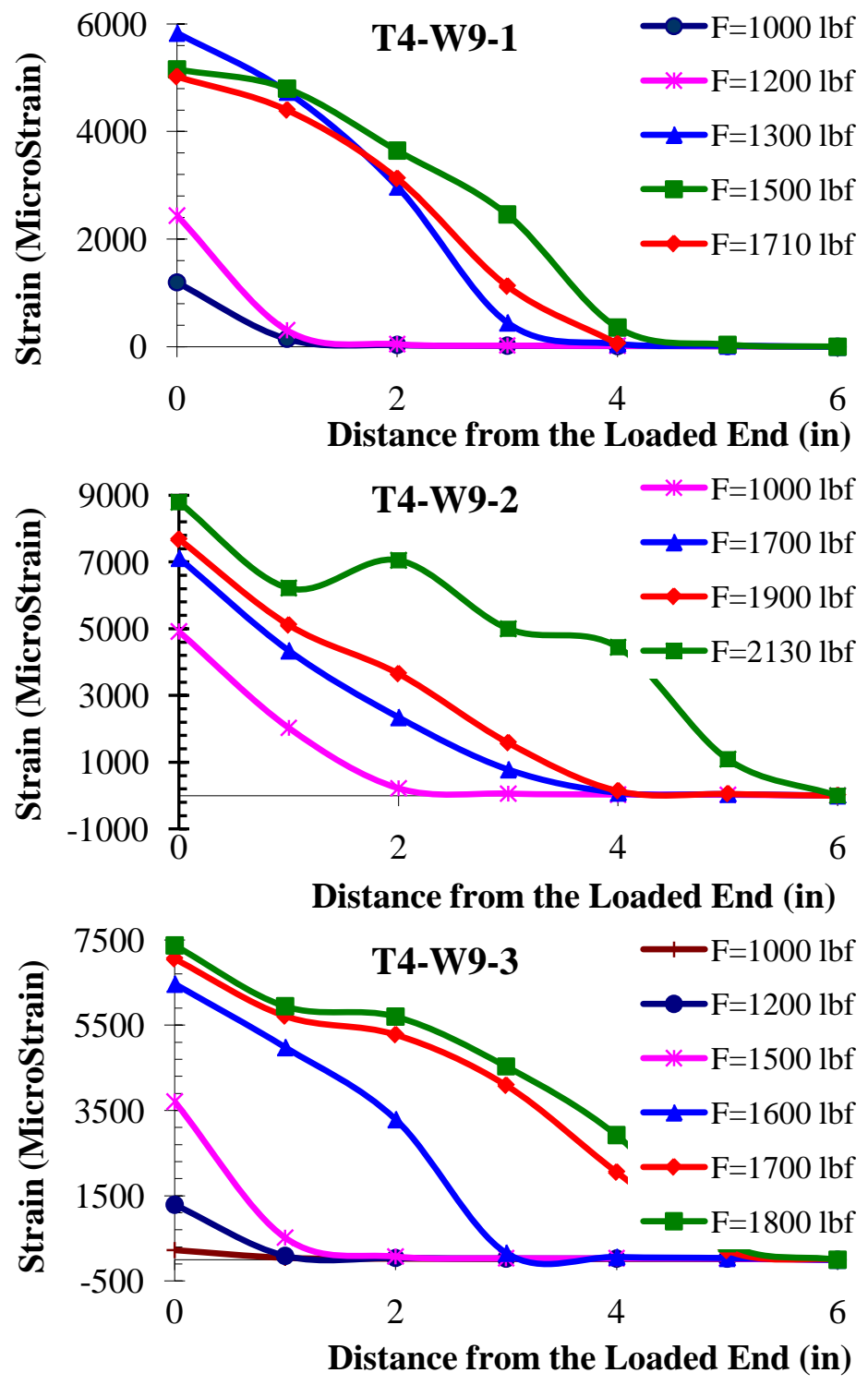

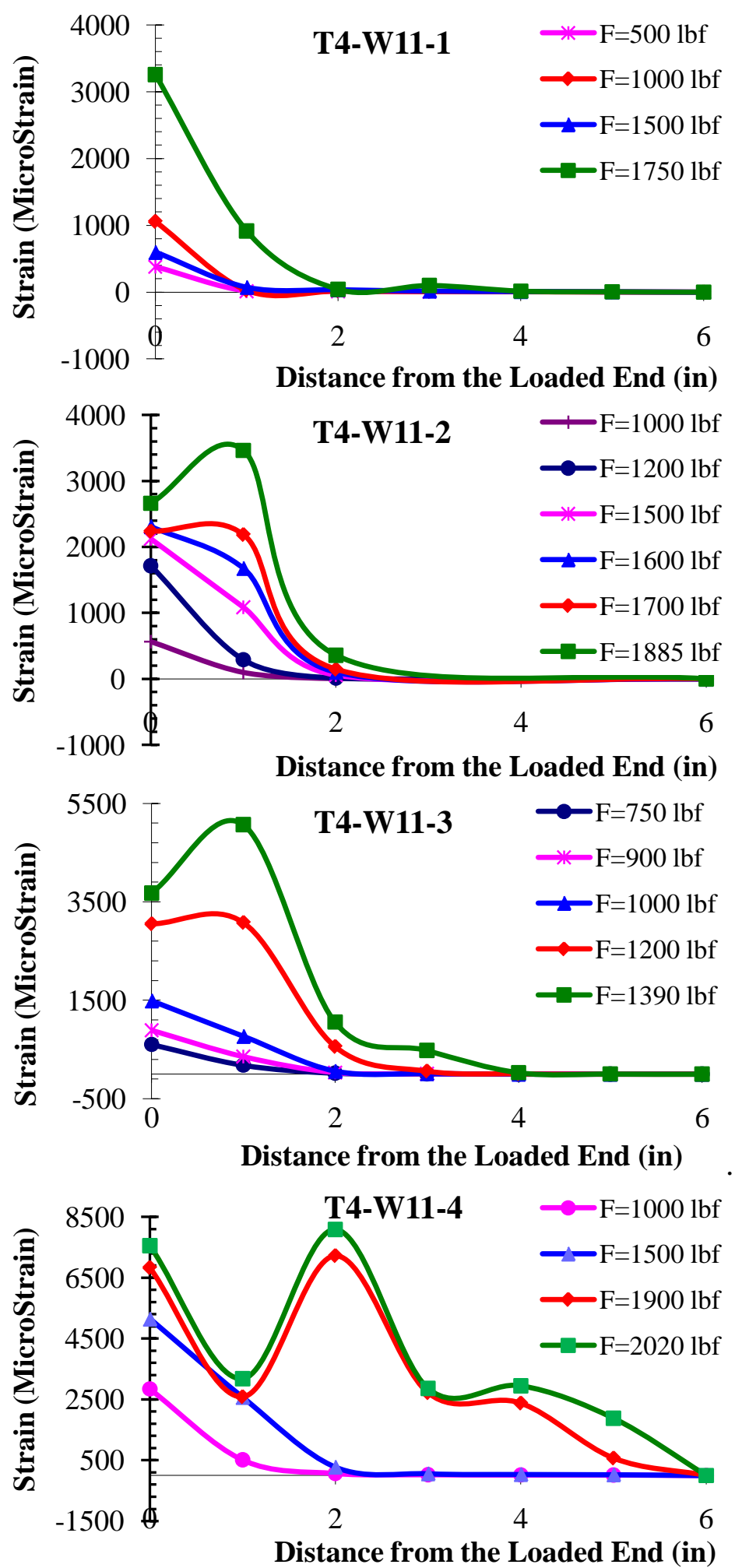

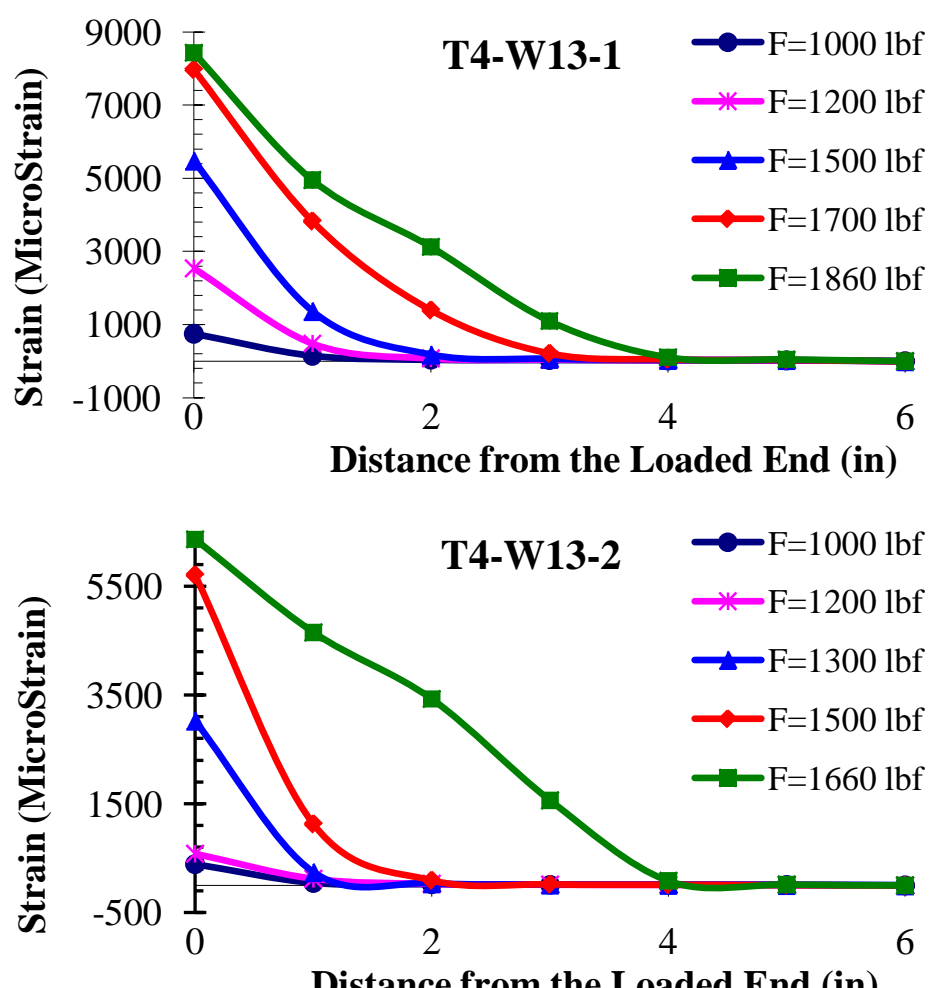

Distance from the Loaded End (in)

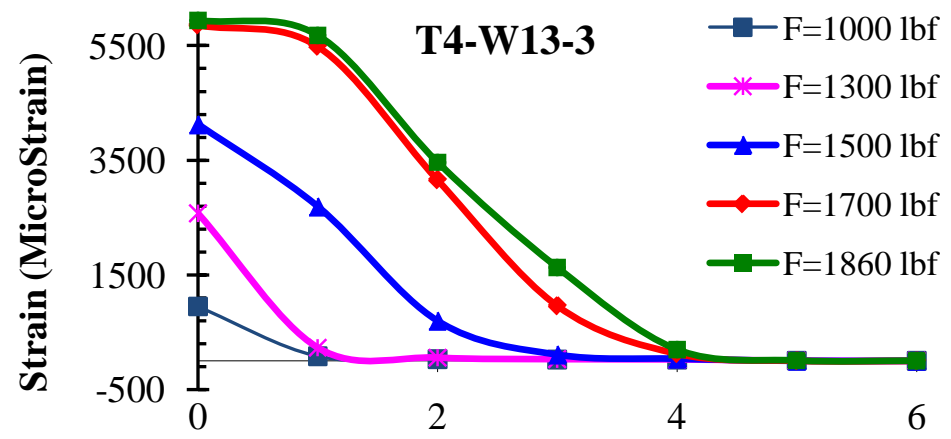

Distance from the Loaded End (in)

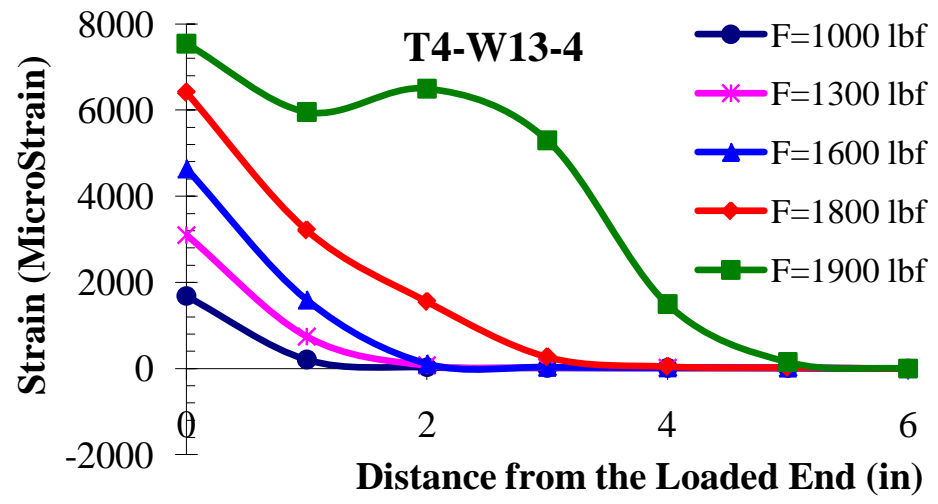




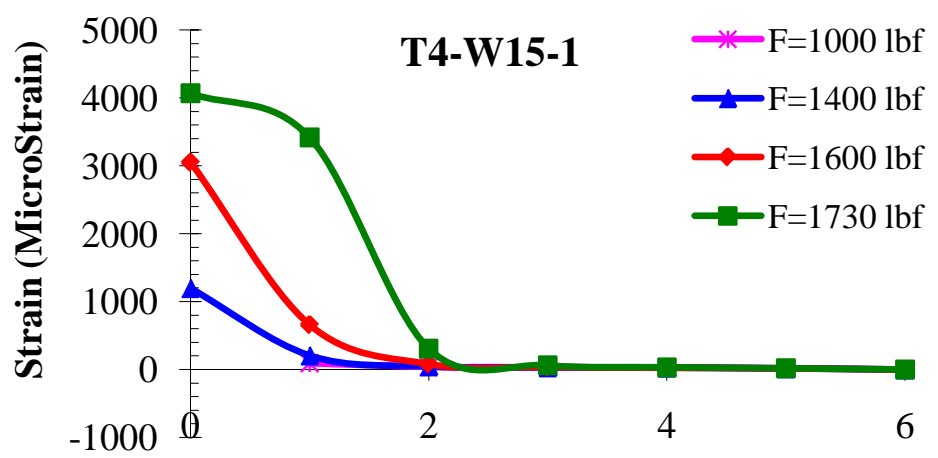

Distance from the Loaded End (in)
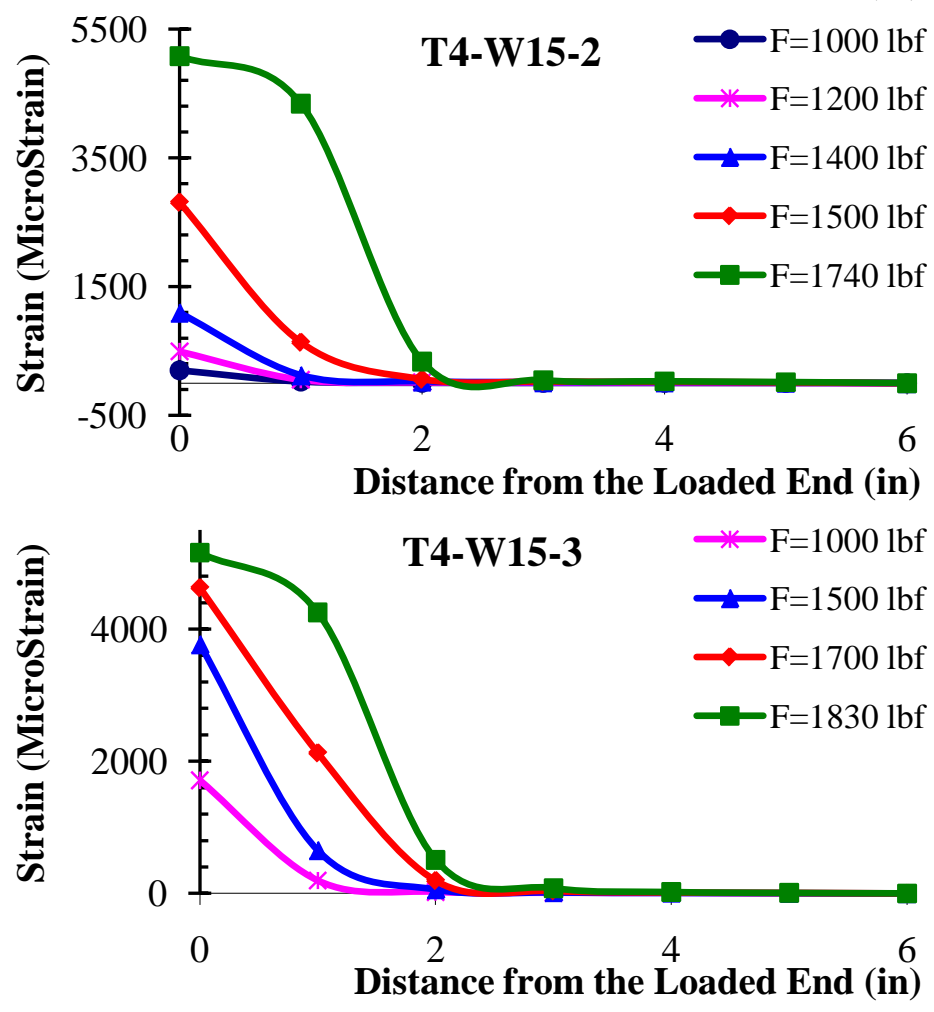


\section{Appendix B: Load- Slip Curves}


T1-W11-02
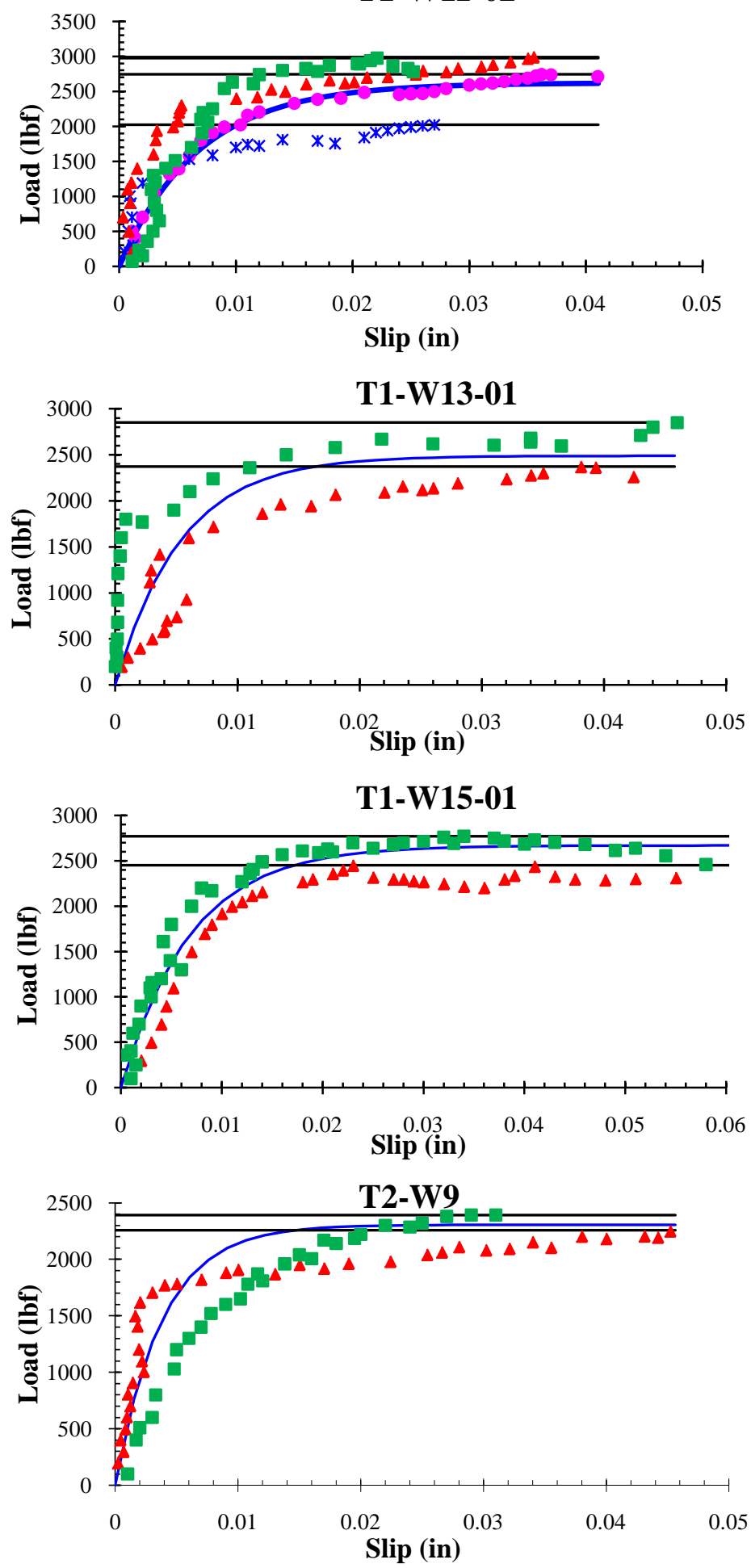

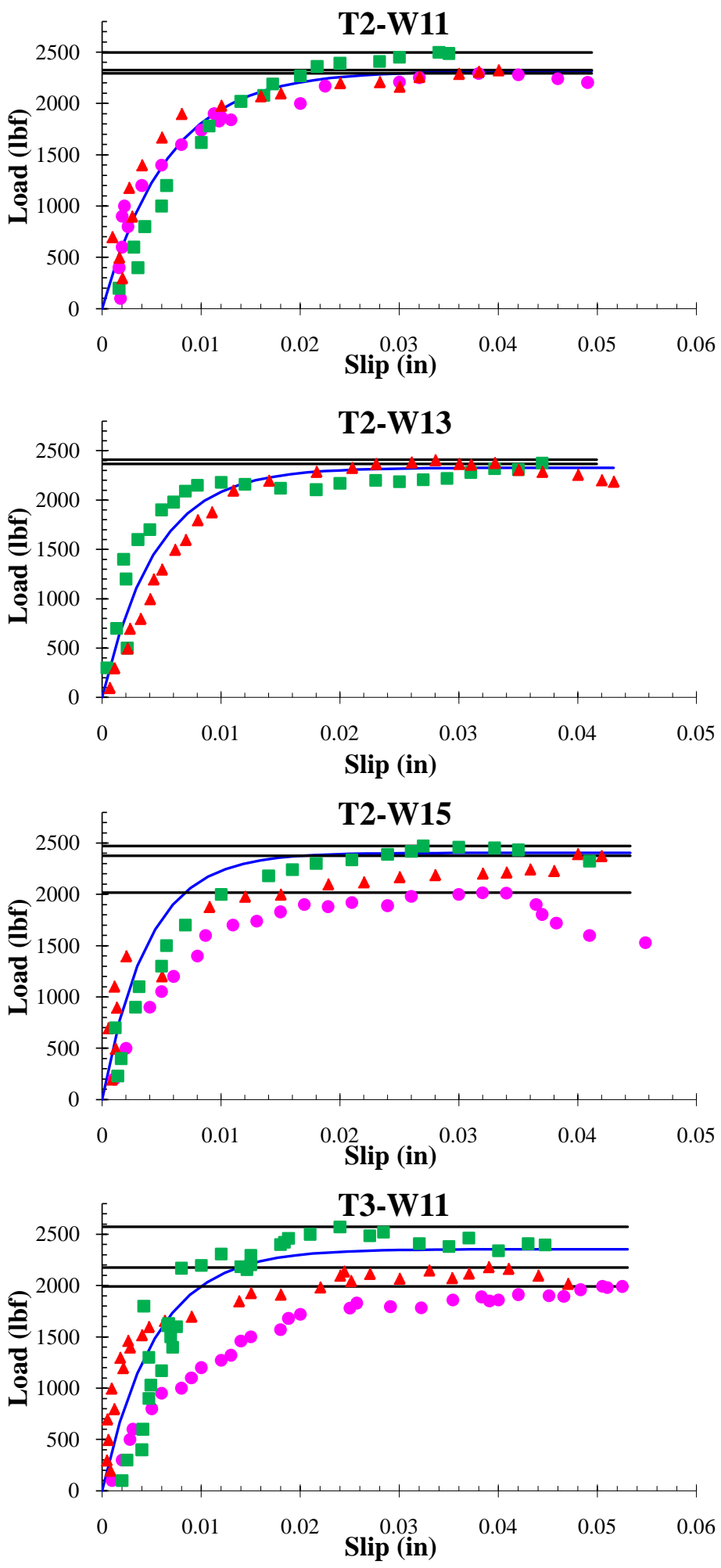

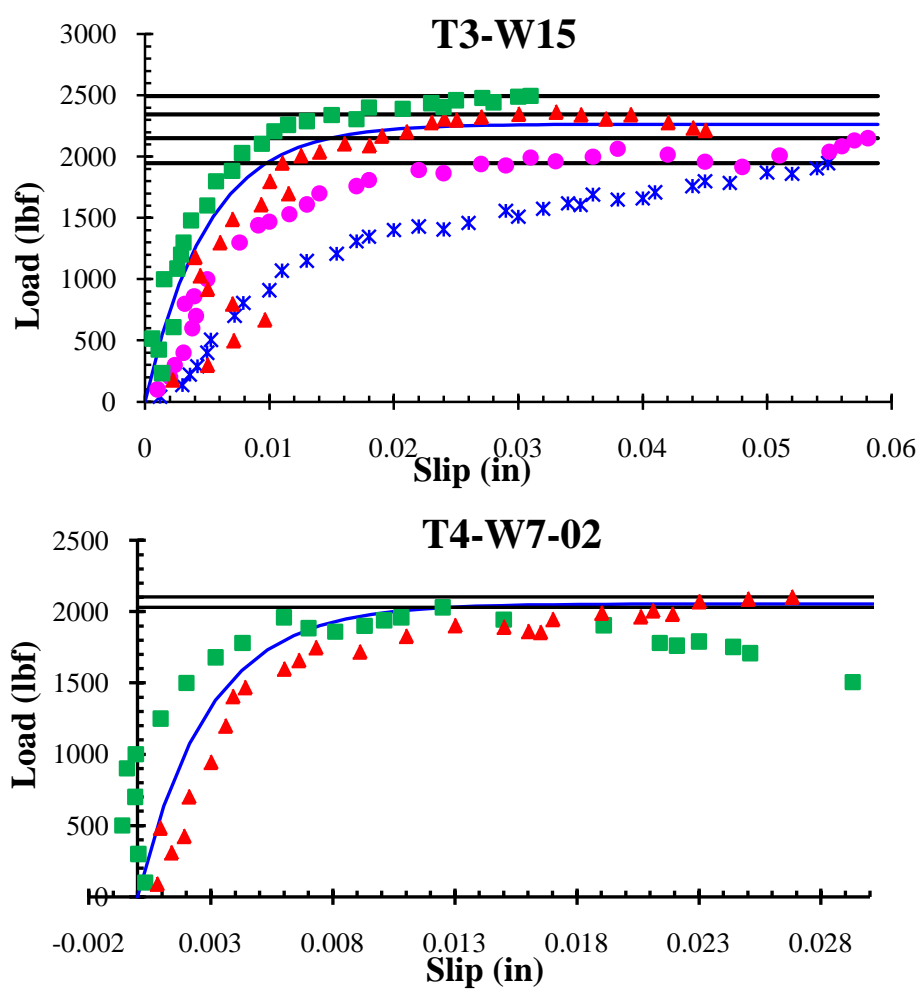

T4-W9-02
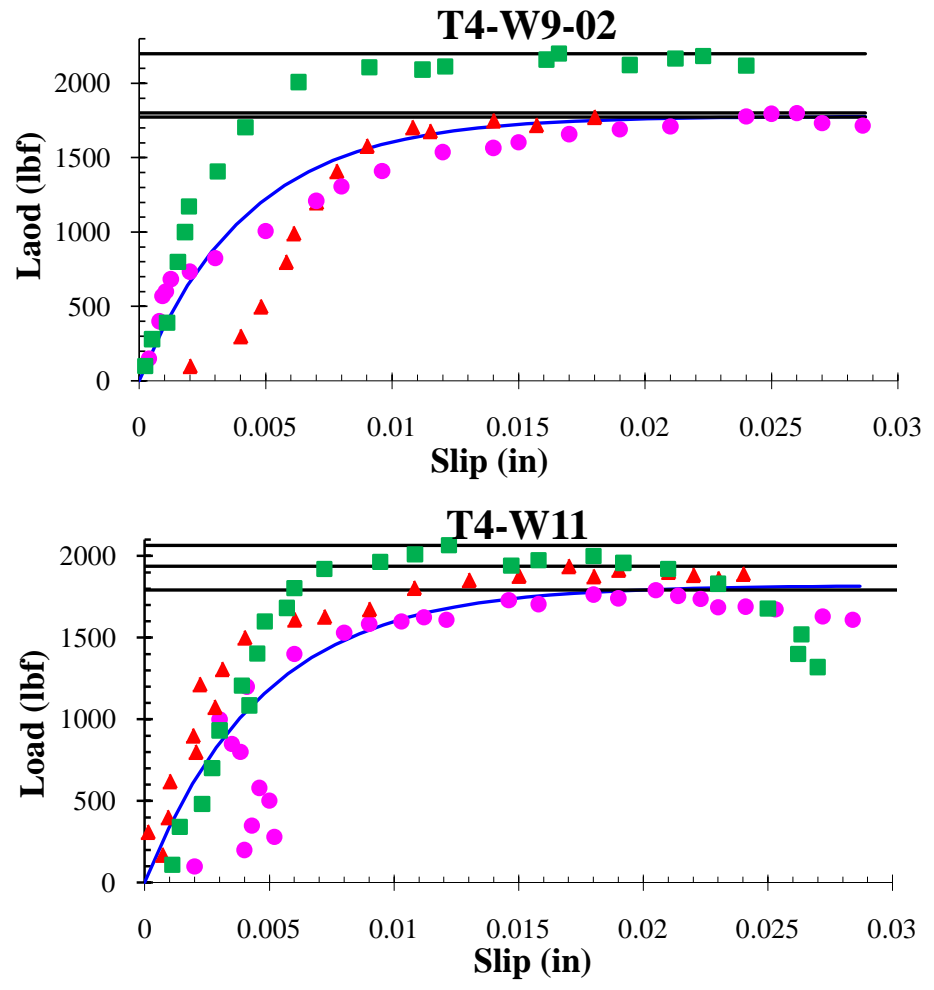

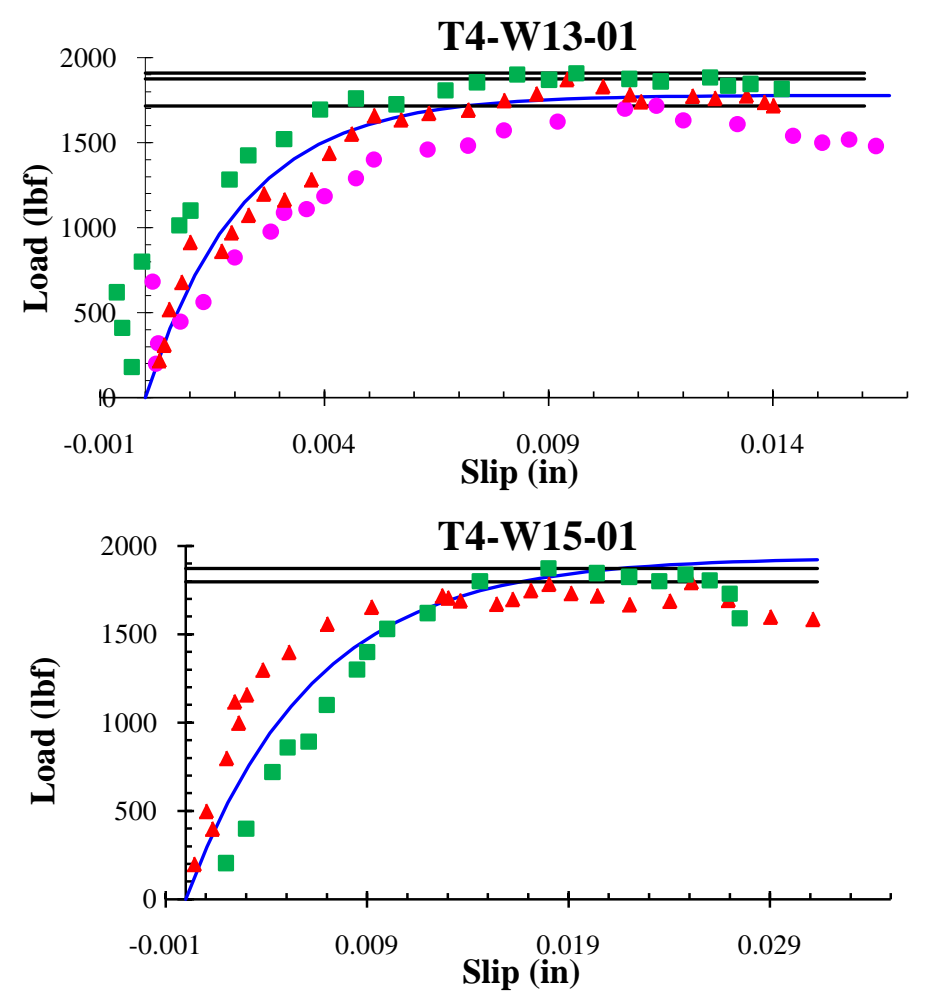

John $\mathrm{H}$ H. 